Unravelling Networks

Causes and Consequences of Decreasing

Connectivity in Bird Migration Pathways

\section{Yanjie Xu}

\section{INVITATION}

To the public defense of my PhD thesis entitled:

Unravelling Networks: Causes and Consequences of Decreasing Connectivity in Bird Migration Pathways

Yanjie Xu

Tuesday, 6 0ctober 2020 1:30 p.m.

Aula, Wageningen University

\& Research

Generaal Foulkesweg I, Wageningen, the Netherlands

Paranymphs Jasper A.J. Eikelboom Shanshan Yang

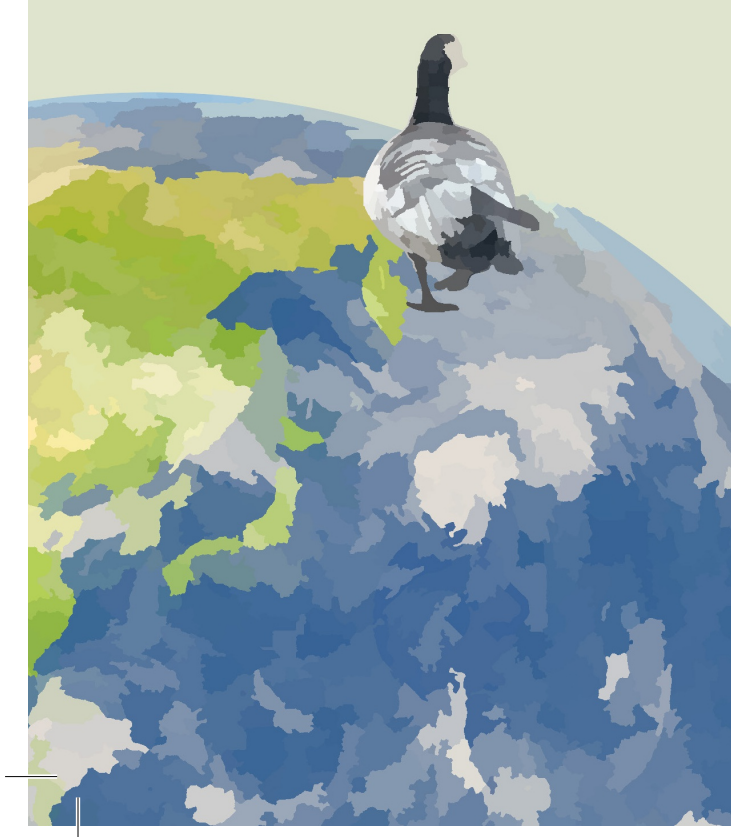




\section{Propositions}

1. The most important prerequisite for conservation of migratory birds is to maintain connectivity of their migratory movements. (this thesis)

2. If the migration network of a bird species breaks down, the size of its total population will decline, but this decline will be delayed.

(this thesis)

3. When the costs are equal, nature preservation is more effective than restoration.

4. Increased efforts to visualize scientific results are needed to improve the impact of science.

5. Productive researchers stay on the left side of the Medawar zone.

6. Quarantine measures to reduce covid-19 transmission have to be weighed against increased transmission risks between humans and wildlife, because of the observed rewilding in human-modified landscapes.

Propositions belonging to the thesis, entitled

Unravelling Networks: Causes and Consequences of Decreasing Connectivity in Bird Migration Pathways

Yanjie Xu

Wageningen, 6 October 2020 


\section{Unravelling Networks}

Causes and Consequences of Decreasing Connectivity in Bird Migration Pathways

Yanjie Xu 


\section{Thesis committee}

\section{Promotors}

Dr W.F. de Boer

Associate professor, Wildlife Ecology and Conservation Group

Wageningen University \& Research

Prof. Dr H.H.T. Prins

Professor of Resource Ecology

Wageningen University \& Research

\section{Co-promotors}

\section{Dr Y. Si}

Associate professor, Department of Earth System Science

Tsinghua University, Beijing, China

Prof. Dr P. Gong

Professor, Department of Earth System Science

Tsinghua University, Beijing, China

\section{Other members}

Prof. Dr M. Naguib, Wageningen University \& Research

Dr M.J.J.E. Loonen, University of Groningen

Prof. Dr K. Kramer, Wageningen University \& Research

Dr T. Mundkur, Wetlands International, Ede

This research was conducted under the auspices of the C. T. de Wit Graduate School for Production Ecology and Resource Conservation (PE\&RC). 


\title{
Unravelling Networks \\ Causes and Consequences of Decreasing Connectivity in Bird Migration Pathways
}

\author{
Yanjie Xu
}

\section{Thesis}

submitted in fulfilment of the requirements for the degree of doctor at Wageningen University

by the authority of the Rector Magnificus

Prof. Dr A. P. J. Mol,

in the presence of the

Thesis Committee appointed by the Academic Board

to be defended in public

on Tuesday 6 October 2020

at $1.30 \mathrm{p} . \mathrm{m}$. in the Aula. 
Yanjie Xu

Unravelling Networks: Causes and Consequences of Decreasing Connectivity in Bird Migration Pathways,

210 pages.

$\mathrm{PhD}$ thesis, Wageningen University, Wageningen, the Netherlands (2020)

With references, with summaries in English and Dutch

ISBN: 978-94-6395-502-7

DOI: https://doi.org/10.18174/529380 


\section{Content}

$\begin{array}{lll}\text { Chapter } 1 & \text { General Introduction } & 7\end{array}$

Chapter 2 Species-dependent effects of habitat degradation in $\quad 21$ relation to seasonal distribution of migratory waterfowl in the East Asian-Australasian Flyway

Chapter 3 Loss of functional connectivity in migration networks induces population decline in migratory birds

Chapter 4 Indicators of site loss from a migration network: anthropogenic factors influence waterfowl movement patterns at stopover sites

Chapter 5 A network approach to prioritize conservation efforts for migratory birds

Chapter 6 Range shifts of migratory hosts facilitate avian influenza panzootics via crossroads of flyways

Chapter $7 \quad$ Synthesis

Bibliography

Summary

Samenvatting

Biography

Affiliation of co-authors

Acknowledgements

PE\&RC Training and Education Statement 
CHAPTER 1 
General introduction 


\subsection{Network theory}

Network theory offers an integrative mathematical framework for understanding a system from its structural characteristics and its functional properties. Networks are constructed with nodes (e.g., individuals, locations, or computers) and edges (linkages between the nodes). Networks have a so-called "ego", or "global" prospective. From an ego perspective, network theory investigates the relationships related to one node while in a global perspective, relationships of all nodes in the network are investigated 2. By calculating ego and global network metrics (Box 1.1), the connectivity of networks, and the contribution of each node to this

Box 1.1 Definitions for a network and their applications in bird migration networks

a. Main components

- Nodes are points in networks which can be a representation of individuals, locations, or communication end points. In a bird migration network, I define nodes as the sites which birds use for foraging, resting, moulting, or breeding.

- Edges are connections between nodes, which represent bird movements between the sites in a bird migration network.

- Modules are groups of nodes which are intensively connected with each other, but sparsely connected to other nodes from a different module in a network ${ }^{1}$.

- Shortest paths are the least-cost routes from one node to the other, in which other nodes in the network may be included as stepping stones ${ }^{4}$.

b. Global metrics (the structural characteristics of an entire network)

- Network size is the number of nodes, which e.g., represent the number of sites used by birds from a certain species during migration.

- Number of edges represent the number of direct movements between sites in a network.

- Average path length is the mean length of all shortest paths, which measures the average cost of moving from one site to the other in a network.

- Graph path length is the maximum length of all shortest paths, which measures the maximum cost of moving from one site to another in a network.

- Transitivity is a generalized clustering coefficient ${ }^{6}$, which measures the strength of sites clustering together.

- Modularity measures the strength of dividing a network into smaller modules based on classified module memberships ${ }^{1,8}$.

c. Ego metrics (the degree to which a site contributes to the connectivity of the network)

- Degree is the number or summed weight of edges connected to a focal node ${ }^{4}$, which measures to what extent a site is directly connected to other sites in a network.

- Closeness is the inverse sum of the (weighted) shortest distances to all other nodes ${ }^{4}$, which measures to which degree a site is closely connected to other sites in a network.

- Betweenness is the number of shortest paths going through a node ${ }^{14,15}$, which measures to what extent a site acts as an stepping stone in a network.

- Bridging centrality is local bridging centrality value ${ }^{17}$, which measures to what extent a site connects different modules in a network. 
connectivity can be quantified from different perspectives. Moreover, concepts and applications of dynamic networks were developed based on network theory. The spatial and temporal dynamics of networks, which can be quantified by the comparison of network structures across space and time ${ }^{3}$, further reflect spatial heterogeneity and temporal evolution of networks ${ }^{5}$.

The first application of network theory was a topological route design for connecting two islands by crossing seven bridges ${ }^{7}$, which was followed by other early applications including electrical circuit models ${ }^{9}$, and transportation network planning ${ }^{10}$. Network theory has been further developed in sociology, investigating social structures as the social ties that link different individuals or group affiliations $4,11,12$. Recently, network theory has been applied to various other fields. For instance, in information science, authors' collaborative relationships in a certain field can be quantified by constructing a co-authorship network and calculating network metrics including degree, closeness, betweenness, and modularity, which are ego and global metrics for measuring network structures (Box 1.1) ${ }^{2}$. In criminology, network analysis can be applied to better understand the relationships between actors involved in illicit markets, and for formulating targeted law enforcement operations. For example, it is possible to identify the crucial actors who act as hubs in a drug market network, which are well-connected to other actors or function as bridges between different criminal groups ${ }^{13}$. In epidemiology, the sources and hubs of global H5N1 transmission can be identified by a network of viral flow ${ }^{16}$, and the transmission process can be analysed by comparing $\mathrm{H} 5 \mathrm{~N} 1$ viral transmission networks with bird migration networks ${ }^{18}$.

In ecology, network analyses have been implemented widely to investigate trophic networks, e.g., food web typologies. The term of 'ecological network' represent specifically an interaction network between organisms (nodes) in the ecosystem, which includes biotic interactions (edges) like mutualism, commensalism, competition and/or predator-prey relationships 19,20 . These networks were used to study how the ecosystem structure influences the functional stability of the system ${ }^{21,22}$, e.g., a higher complexity in food webs is often related to a higher stability of the system ${ }^{23}$. Besides these biotic interactions, network theory can also be used in other topics of animal ecology, to quantify e.g., social interactions and movement behaviours 24,25 . As for instance, Snijders et al. compared explorative 
behaviour of great tits (Parus major) with different social network position by investigating how personality affects social position within their networks ${ }^{26}$. Moreover, dynamics in social structures of animal populations amongst years and seasons can be systematically monitored by a network analysis, as for example the social networks among female Asian elephants (Elephas maximus) at pair (ego), companion (module), and population (global) levels 27. Furthermore, studying turnover in social networks after introducing disturbance enables a better understanding of the adaptivity and resilience of animal populations to environmental changes ${ }^{28-30}$. Similar insights can also be derived by investigating animal movement networks. As for instance, the dynamic cattle (Bos taurus) networks illustrated spatio-temporal heterogeneity through differences in timings and locations, under influence of forage conditions and other factors 5 .

Several studies have applied network theory to investigating movement ecology of resident birds, like the Takahe (Porphyrio hochstetteri; a flightless rail species from New Zealand) ${ }^{31}$ and weaver birds ${ }^{32}$, to evaluate individual movement patterns and network use in relation to differences in habitat configuration. Calder et al. ${ }^{32}$ found that the movements of weaver birds in Cape Town, South Africa, was highly clustered, and was sensitive to the loss of wetland habitats. Grange et al. ${ }^{31}$ constructed Takahe movement networks from 2007 to 2011, and showed that an increase in their movements was associated with increasing network size and number of edges (Box 1.1) ${ }^{31}$. Furthermore, Grange et al. 31 identified hubs and sources in the transmission pathways of avian infectious diseases by analysing ego metrics for such movement networks of host species. Bird movement networks can also be derived from the network of their habitat patches, as for instance, Gil-Tena et al. ${ }^{33}$ showed that the colonization by woodpeckers expanded with increasing connectivity of different forest patches in Mediterranean Europe. Monitoring dynamics of such bird movements on the basis of a network perspective provides integrative insights for conservation of focal species moving between fragmented habitats.

\subsection{Bird migration network}

Migration is a regular seasonal movement of e.g., birds, mammals, or fish. Within each annual life cycle, migratory birds in the northern hemisphere travel to their breeding grounds (northward migration) and return to their non-breeding grounds (southward migration). Although they have great ability to fly over long distances across latitudes, most of them are not able 
to reach their breeding grounds directly from their wintering grounds (and vice versa) ${ }^{34}$. Stopover sites are stepping stones for resting and refuelling during migration, which are critically important for their migration success and population sustainability ${ }^{34-36}$. Thus, in this thesis, I define the breeding, non-breeding, and stopover sites as nodes and the movements between these sites as edges in these bird migration networks.
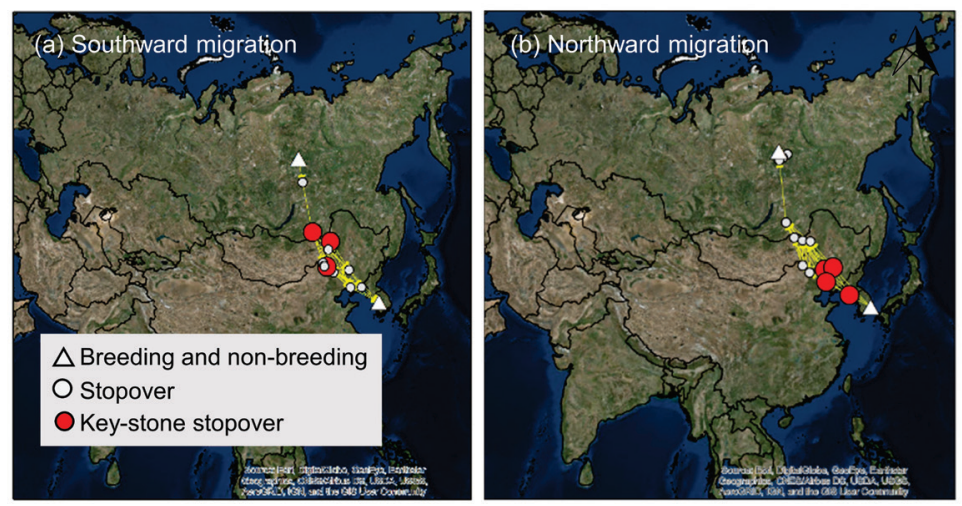

Figure 1.1 An example of bird migration networks. Southward and northward migrations of whooper swans (details in Chapter 5).

It is important to understand how environmental factors (i.e., climate, land use, human disturbance) affect movements and population dynamics of migratory birds from a network perspective. Firstly, the importance of stopover sites may differ from each other, such as through differences in habitat availability and/or position in the migration network. Habitat loss in a key stopover site in a migration network may lead to cascading effects, and contribute to a population decline in birds ${ }^{35}$, or can even trigger a network collapse, which isolates breeding and non-breeding grounds and completely impedes their migration ${ }^{37}$. Secondly, for migratory species, body conditions and/or energy intake in one season may affect or be affected by those in another season (carryover effect) ${ }^{38}$. As for instance, to cope with the advanced spring in the Arctic breeding ground, barnacle geese (Branta leucopsis) skipped or reduced staging time in some stopover sites during northward migration ${ }^{39}$. However, insufficient body reserves acquired at stopover sites forced them to spend some time to refuel their body stores at their breeding grounds before egg laying, which resulted in a mismatch between the peak in food quality and gosling hatching time 39,40.

Network theory provides advanced tools for measuring connectivity of bird migration networks, taking interactive effects of habitat conditions 
across seasons into account. Following the definition of Webster et al. ${ }^{41}$, migratory connectivity quantifies the strength of links between breeding and non-breeding sites. Migrants with strong migratory connectivity migrate from one breeding site to one non-breeding site, while those with weak connectivity migrate from one breeding site to several non-breeding sites 41 . Migratory connectivity enables testing the consequences of migrating with different migratory strategies regarding to their breeding and non-breeding ranges, e.g., species with strong migratory connectivity may be less resilient to climate change but more resilient to anthropogenic habitat modification 42. However, migratory connectivity is measured by ranges of breeding and non-breeding grounds while the stopover sites along migration routes are not accounted. By incorporating the alternative paths, non-random structures (e.g., clustering, modularity in Box 1.1), and relative importance of the connections between stopover sites with tools derived from network theory ${ }^{43}$, connectivity of a bird migration network better summarizes the structural and/or functional features of migration networks. Investigating this connectivity with network theory provides insights for systematic management plans to improve conservation actions for migratory birds. Specifically, better understanding bird migration networks enables identifying the importance of certain stopovers for maintaining the connectivity within the network. Meanwhile, the study of connectivity of migration networks fills an important knowledge gap for better understanding dynamics and stability of bird migration (Figure 1.2) and the early-warning signals of population decline and/or network collapse (Figure $1.2 \mathrm{c})$. Thus, the connectivity of a migration network investigated in this thesis is defined as the degree to which the sites along the migration routes facilitate bird movements, which is determined by a combination of ego and global network features, landscape connectivity of habitat patches, and the probability that a bird species can no longer migrate between breeding and non-breeding sites.

There are only few studies that investigated bird migration networks. Merken et al. ${ }^{44}$ analysed and simulated the spatial migration networks along the Black Sea-Mediterranean Flyway across the Sahara. This study revealed that the trans-Sahara migration flyway for waterbirds was wellconnected, and the quantification of the importance of each wetland in the flyway is useful for the evaluation of crucial sites in this migration network. Bellisario et al. 45 further investigated the current and future connectivity of wetland sites within the Natura 2000 network, and predicted a sudden 
collapse of this protected network for waterbirds under climate change. Both studies identified suitable wetland sites as the nodes within the migration network, and modelled the edges by distances between the sites, which may be biased by the species-specific rules for habitat selection and movement patterns. In one empirical study for North American waterfowl, speciesspecific flyways were identified by a network module detection algorithm (Box 1.1) from observations of movements ${ }^{46}$. These biological-based migration routes better identify bird movements and migration corridors compared to historical documented flyways ${ }^{47}$. Another case study analysed the migration networks of oriental storks (Ciconia boyciana), and detected potential risks for network collapse by the removal of important stopover sites along their migration route. The authors showed that these storks would not be able to reach their wintering sites along the Yangtze River if they would lose stopovers in the Bohai Bay during autumn migration ${ }^{37}$.

\subsection{Dynamics of bird migration networks and conserving network connectivity}

Habitat loss and disturbance currently pose great threats to wildlife 48,49 . Although some bird species may take advantage of human modified landscapes (e.g., geese in European and North American farmlands) ${ }^{50}$, the habitats utilized by many migratory birds along their migration routes are deteriorating and negatively affect avian populations due to rapid land use changes and climate changes ${ }^{35,45,51}$, and connectivity of migration networks may consequently decrease. Climate warming and anthropogenic habitat loss may change the structure of migration networks in many ways. On one hand, migratory birds may shift their ranges e.g., northwards in North America or east-northwards in Europe when the main driver for the changes in these migration networks is climate warming ${ }^{52-54}$. On the other hand, they can lose sites from a network under high human disturbance or when anthropogenic habitat modification takes place ${ }^{42}$. Both drivers may reduce connectivity in bird migration networks ${ }^{42}$, but the plasticity and/or vulnerability of different species with different migration strategies to this different agents for connectivity loss may differ.

As a result of different disturbance agents to migration networks and their species-specific effects, a large proportion of migratory species around the world are experiencing population declines in recent decades $49,55,56$. This may be partly explained by decreasing connectivity of their migration 
networks. Habitat loss may reduce the number of suitable sites in the network, which results in a reduced connectivity and a limited number of alternative routes for migration (Figure 1.2b). Consequently, migratory birds may experience a higher resource competition, longer non-stop flights, and reduced flexibility to environmental changes 57 . Furthermore, if habitats continue to degrade, a migration network may collapse, and bird movements between breeding and non-breeding grounds can be totally impeded (Figure $1.2 \mathrm{c})$.
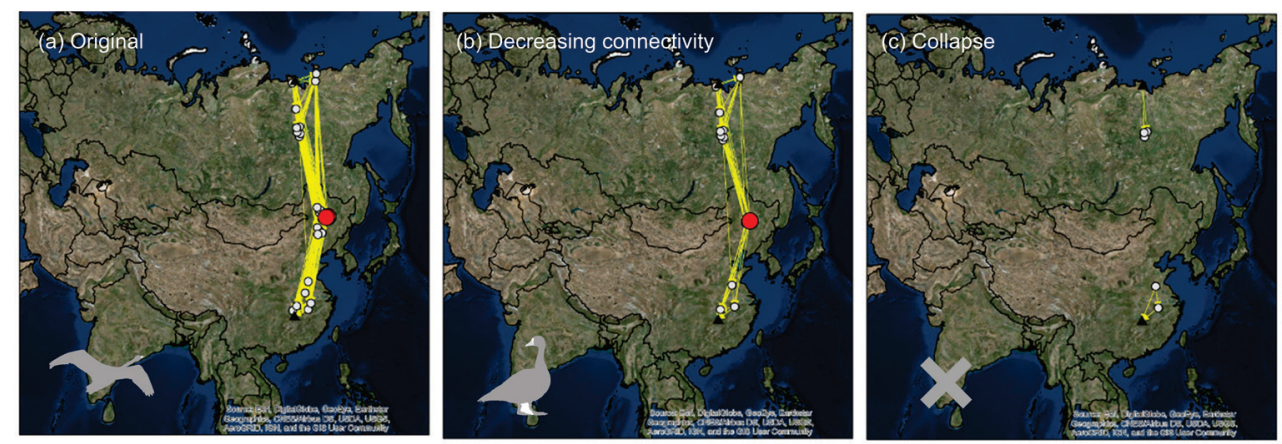

Figure 1.2 Simulated impact of habitat loss on a bird migration network. Using southward migration of greater white-fronted geese as an example

Birds are excellent bioindicators of ecosystem health under habitat disturbance ${ }^{58}$. Investigating the mechanisms for rapid population declines in birds from a network perspective can provide early-warning signals of ecosystem degradation, and facilitate targeted conservation plans for wildlife. Furthermore, the knowledges about dynamics of movement networks can facilitate better surveillance and forecasting of the impact of avian migrations on e.g., avian disease transmission 59,60. However, the understanding of changes in migration networks under recent trends of climate and land use changes, and how this affects population dynamics of migratory birds remains limited.

Migratory birds seasonally utilize a variety of habitats, patchily distributed over a large spatial extent 34,36 . Thus, a better understanding for their population dynamics and a targeted conservation strategy for birds should take the whole migration network into consideration. For instance, the population decline of a migratory species in the Arctic breeding sites can be related to factors operating at non-breeding grounds or by the loss of stopover sites 39,61 . As predicted by a theoretical study, migratory birds spend six times more time at stopover sites than that in flight during their seasonal migration ${ }^{62,63}$. The availability and suitability of stop-over habitats 
affect not only the migration, but also the breeding success and the survival probability of adult birds ${ }^{64}$. Especially, for capital breeders (like some geese species) who store energy for reproduction ahead of reaching their breeding grounds 65,66 , their breeding success highly relies on the quality of foraging and roosting in stopover sites during northward migration 67.

Quantifying the dynamics of stopover sites and the responses of migratory birds to stop-over quality driven by landscape configurations provides knowledge about the risks that a migration network may collapse. Habitat availability and suitability both determine whether a site can be used as a stopover for migratory birds, and influence their foraging, migration, behavioural decisions, and subsequently their breeding success 44,68. Habitat suitability is under influence of environmental factors and determines to what extent e.g., a wetland site meets the habitat requirements of a waterbird species ${ }^{69}$. Habitat availability determines whether a habitat site can be reached by a species during their migration, which is influenced by the connectivity of habitat patches along their different migration routes. Generally, when a site is well-connected to others, it is available for that migratory species ${ }^{70}$. The declined quality of stopover sites and the loss of connections among sites can weaken the linkage between their breeding and non-breeding sites ${ }^{71,72}$, and may subsequently lead to collapse of migration networks.

Identifying keystone stopover sites is a crucial step for maintaining a well-connected network of sites along the migration pathways of birds. According to theoretical studies, losing a keystone stopover site can breakdown a bird migration network completely (Figure 1.2) ${ }^{37}$, and consequently trigger population declines. Habitat loss may bring a magnified impact on migratory species who rely on keystone sites in their migration network ${ }^{73}$. Thus, quantifying site importance from a network perspective enables targeted conservation actions for migratory birds by prioritizing conservation efforts for these keystone sites.

\subsection{Thesis outline}

In this thesis, I analysed the migration networks of $>50$ migratory waterfowl species. In an era of rapid global change, the connectivity of current migration network may be decreasing and the network could collapse due to degradation or loss of important stopover sites or connections between stopover sites under rapid environmental changes and intensive human 
disturbances. Considering land use and climate changes, I investigated the dynamics of these species-specific networks (Chapter 2,3,4,5) and predicted the potential risks for network breakdown (Chapter 4,5). A decreasing connectivity in bird migration networks may be an important predictor for population declines in birds. A changing structure of migration networks under environmental changes can also result in an unhealthy ecosystem, e.g., with rapid disease transmission. Thus, I tested the degree to which the changing connectivity in bird migration networks affect their population dynamics (Chapter 3), the risks of species extinction (Chapter 5), and the ecosystem consequences of the changes for e.g., disease transmission (Chapter 6). Conserving keystone stopover sites quantified from a network perspective may be an effective and efficient strategy for maintaining or improving the connectivity of bird migration networks. Synthesizing all these results, I propose a novel framework for assessing migration networks, which enables prioritization of sites for conservation of migratory species (Chapter 5,7). Consequently, this thesis offers novel insights into how climate and land use changes impact migratory birds and provides effective strategies for targeted conservation.

In chapter 2, I monitored the pattern of habitat loss and fragmentation from 1992 to 2012 in wetland sites along the migration corridors of eight waterfowl species. I modelled the distributions of these waterfowl species and tested the degree to which they are affected by these habitat degradations. Furthermore, I tested the differences between species using different migration corridors to explore species-specific effects of habitat loss.

In chapter 3, I observed a weakening connectivity in migration networks of East-Asian waterfowl species from 1992 to 2015. I tested how this connectivity loss, along with previously-considered predictors for population declines in migratory birds, affect population dynamics of eight waterfowl species. I proposed a novel and crucial predictor for population declines of migratory birds: loss of functional connectivity in migration networks, which outperforms previously-considered predictors for population dynamics of migratory birds.

In chapter 4, I investigated the movement patterns of greater whitefronted geese (Anser albifrons) in different stopover sites with different habitat configurations. Habitat modifications from human activities can provide opportunities for waterfowl by e.g., intensifying their agriculture 
(i.e., providing suitable foraging sites) while these activities can also negatively affect waterfowl habitats by destroying wetlands and disturbance from, e.g., heavy road traffic. I tested the direction and extent to which environmental factors related to habitat configurations affect waterfowl movements at their stopover sites during migration. The identified factors can be monitored and can maybe serve as indicators of the probability that a site may be lost from a migration network.

In chapter 5, I constructed seasonal migration networks for four Asian waterfowl species empirically by processing satellite tracking data as to define the nodes and edges of the network. To better understand to what extent site-specific variables and network metrics that characterize the site with regard to its position in the network, contribute to the breakdown of migration networks, I quantified the risk of migration network collapse and identified keystone sites in migration networks (i.e. sites that are pivotal in maintaining the network connectivity and are experiencing habitat loss). I found that migration network connectivity decreased rapidly when removing sites in order of betweenness (Box 1.1), and that nearly half of the keystone sites are not designated as protected area.

In chapter 6, I investigated how global changes affect changes in the structures of migration networks, and potentially accelerate the spread of bird-borne infectious diseases. Migratory birds carry avian influenza viruses around the world through the crossroads of flyways (i.e., locations where different populations mix and viruses can jump hosts). I constructed movement networks for 47 migratory waterfowl species in North America and Eurasia and mapped intra-species and inter-species crossroads between flyways. I observed changes in migration networks from 1950 to 2019 , partly resulted from range shifts of these migratory species. Subsequently, crossing intensities within and between species increased, which could increase the risk of long-distance transmissions and broadscale outbreaks of infectious diseases.

In chapter 7, I synthesized the various consequences from reduced connectivity of bird movement networks for bird populations and ecosystem services. Conservation actions at present are often driven by site-specific properties, e.g., the amount of birds that visit a particular site. However, the importance of a site with regard to its position in the network is often ignored and remains at a qualitative level. In this chapter, I proposed a quantitative framework for the definition of site importance in bird 
movement networks, which may facilitate efficient and effective conservation actions that contribute to the connectivity of migration networks. 



\section{Species-dependent effects of habitat degradation in relation to seasonal distribution of migratory waterfowl in the East Asian- Australasian Flyway}

Xu Y., Si Y., Yin S., Zhang W., Grishchenko M., Prins H. H. T, Gong P., de Boer W. F. Species-dependent effects of habitat degradation in relation to seasonal distribution of migratory waterfowl in the East Asian-Australasian Flyway. Landscape Ecology 34, 243-257 (2019) 


\section{Abstract}

Migratory species' resilience to landscape changes depends on spatial patterns of habitat degradation in relation to their migratory movements, such as the distance between breeding and non-breeding areas, and the location and width of migration corridors. We investigated to what extent the impact of habitat degradation depended on the seasonal distributions of migratory waterfowl. Using logistic regression, we selected wetland sites for eight waterfowl species in the East Asian-Australasian Flyway (EAAF) by calculating the probabilities of species occurrence per wetland site in relation to environmental factors. We quantified landscape metrics related to habitat degradation within these wetland sites. We used general linear models to test for differences in the effects of habitat degradation on waterfowl species with different migration extents and at different latitudes. The patterns of habitat degradation differed spatially across the EAAF and affected species to a different degree. Species with shorter and broader migration corridors (Anser cygnoid and A. anser) could benefit from improved habitat conditions in the west of the EAAF. Species with longer and narrower migration corridors (Cygnus columbianus, A. fabalis, A. albifrons, A. erythropus, Anas crecca, and Anas acuta) were under higher risk of habitat degradation in the coastal regions of China and Japan. Migratory species with longer and narrower migration corridors are more affected by habitat degradation, because they might have fewer alternative stopover sites at similar latitude. Our findings improve the understanding of species-specific effects of environmental changes on migratory species, and defines critical and endangered wetland sites, and vulnerable species. 


\subsection{Introduction}

Habitat loss is one of the most important factors causing population declines in migratory birds ${ }^{74}$. Habitat degradation along migration routes has been linked to decreases in populations of a number of migratory bird populations 35,73 . Wetlands, the main habitat for migratory waterfowl species, are among the most threatened habitats worldwide, and nearly half of the world's wetlands have disappeared as a result of the expansion of human activities 75,76 . China has lost 33\% of its wetland area from 1978 to 2008 , although the trend of wetland loss is slowing down lately 77 . Hence, in the last decades, ecosystem service values of natural areas have declined substantially as a consequence of wetland loss and degradation 78 .

Landscape composition and configuration of suitable habitats affect species occurrence and richness ${ }^{79-82}$. Availability of wetlands and waterbodies, wetland size, and wetland connectivity positively influence waterfowl species occurrence and species richness, while wetlands in proximity to rice fields, total rice field area, and wetland isolation have negative effects 80,81 . Therefore, waterfowl habitat degradation can be quantified by land cover changes and dynamics in landscape variables of wetland sites along migration corridors ${ }^{83,84}$. However, because of limited attention to the spatio-temporal dynamics of wetland sites along migration routes 85 , it is currently unknown how current trends of habitat degradation influence migratory waterfowl species.

The East Asian-Australasian Flyway is one of the nine major waterbird flyways globally. The flyway holds over 50 million migratory waterbirds, including 51 threatened or near-threatened species. Because of the loss and degradation of suitable habitats, resulting from rapid economic development and human disturbance, population sizes for many waterfowl species in the northern part of the flyway have declined rapidly 55,86-89.

The delineated range of the East Asian-Australasian Flyway is rather broad, so the species that use it display considerable variability in the spatial patterns of their breeding, non-breeding, and stopover sites. For instance, the swan goose (Anser cygnoid) breeds in both eastern and western Mongolia 90, while the greater white-fronted goose (Anser albifrons) is an Arctic-breeding migrant with a distribution extending to the Lena Delta, Siberia. However, the non-breeding grounds of the greater white-fronted goose in the Yangtze River Basin overlap with those of swan goose ${ }^{89}$. The falcated duck (Mareca falcata) uses both the eastern and central parts of the 
East Asian-Australasian Flyway, while the common teal (Anas crecca) is restricted to the eastern part of the flyway ${ }^{91}$.

The spatial extent of these waterfowl species' seasonal distributions probably influences the degree to which they are affected by habitat degradation. For instance, population sizes of long-distance migratory species decline more rapidly than those of short-distance migratory species 92. Independently of the distance of migration, species with broader dispersal ranges are less prone to population declines compared to those whose ranges are restricted, because of spatial variation in habitat degradation 56 . In addition, the underlying patterns of habitat loss also make a difference in species-specific consequences of habitat degradations, e.g., a small amount of habitat loss in certain crucial stopover sites can trigger severe impacts 93,94. The resilience of waterfowl species to environmental changes varies because of spatial patterns in habitat degradation and differences in the species' seasonal distributions. However, habitat degradation has not been analysed for its species-specific effects as a consequence of the spatial variation in migration patterns.

We quantified the spatial patterns of habitat degradation in wetland sites, in relation to the seasonal distributions of eight waterfowl species. Wetland sites can be located in breeding grounds, non-breeding grounds, or stopover sites in a species' migration route. First, we selected all wetland sites where each species was likely to occur in the distribution ranges of each waterfowl species based on the modelled relationships between species occurrence and environmental factors (hereafter suitable wetland sites). Second, within the ranges of suitable wetland sites, as metrics of habitat degradation, we quantified the availability of water area, grassland, and wetland, and quantified wetland fragmentation and isolation, and changes in agricultural resources. Finally, we explored the species-specific effects of habitat degradation in relation to the species' migratory extents. The risk from habitat degradation is determined by how the species' distribution overlaps with the spatial distribution of habitat changes. We expect that migratory species with a longer and narrower migration corridors are more likely to be affected by habitat degradation. The results can provide a better understanding of the underlying mechanisms of how environmental changes affect different migratory species, so that targeted conservation plans can be developed for critical and endangered wetland sites and vulnerable species. 


\subsection{Methods}

\section{Study area}

The East Asian-Australasian Flyway identified by the global monitoring program of Wetlands International stretches across 22 countries, covering East Asia, Southeast Asia, Australia, and New Zealand, and northern areas from the Taimyr Peninsula in Russia to Alaska. Unlike Arctic-breeding shorebirds that spend the non-breeding season in Australia and New Zealand, most of the Arctic-breeding waterfowl in the East AsianAustralasian Flyway migrate only as far south as China ${ }^{95}$. We focused on the waterfowl populations overwintering in the Yangtze River Basin, one of the most important non-breeding grounds in the flyway. Therefore, the study area extended from the Yangtze River Basin to the northern part of the East Asian-Australasian Flyway (Appendix S2.1). Overall, the study area overlaps with six countries: China, Mongolia, North Korea, South Korea, Japan, and Russia.

\section{Study species}

The wetlands in the Yangtze River Basin are key non-breeding sites of eleven goose, swan, and dabbling duck species ${ }^{87}$, including tundra swan (Cygnus columbianus), swan goose, bean goose (Anser fabalis), greater white-fronted goose, lesser white-fronted goose (Anser erythropus), greylag goose (Anser anser), falcated duck, Baikal teal (Sibirionetta formosa), common teal, spotbilled duck (Anas poecilorhyncha), and northern pintail (Anas acuta). Eight of the eleven species were included in our analysis. Falcated duck, Baikal teal, and spot-billed duck were excluded because of a lack of detailed information about their breeding distribution 95 .

\section{Data}

Bird data. Breeding and non-breeding ranges of the eight waterfowl species were obtained from bird species distribution maps of the world (v5.0), produced by BirdLife International ${ }^{95}$. Information on the occurrence of the eight Anatidae species within the study area was obtained from the eBird citizen-science database: eBird Basic Dataset (v1.5), which provides species scientific name, population count, latitude, longitude, and date and time of bird observations 96,97. All records from 1992-2016 were included in the analysis, except for data that were not verified by eBird editors. Duplicate records of the same species, location, date, and time of observations were excluded. The records of the eight study species with in the study area were included in the analysis. In total, there were 89 locations with observations 
of greylag goose, 197 for swan goose, 173 for bean goose, 357 for greater white-fronted goose, 57 for lesser white-fronted goose, 223 for tundra swan, 408 for common teal, and 1110 for northern pintail within the study area.

Data for environmental factors. The polygons of lakes, reservoirs, and smaller water bodies (called 'wetland sites' here) with a surface area $\geq 0.1$ $\mathrm{km}^{2}$ were obtained from the Global Lakes and Wetlands Database ${ }^{98}$. The 500-m-resolution elevation data was obtained from Jonathan de Ferranti's Digital Elevation Data site, which combines data from multiple sources, including ASTER Global Digital Elevation Map (ASTER GDEM), gap-filling Shuttle Radar Topography Mission (known as SRTM), and contour maps ${ }^{99}$. The area of food resources (grassland and cropland) around each lake was derived from the ESA CCI 300-m global land cover products (v2.0.7) of the year 1992100 .

Land cover data for landscape metrics. We used land cover maps for 1992 and 2012 from the European Space Agency (ESA) CCI 300-m annual global land cover products 100 to quantify the spatial patterns of habitat degradation. The land cover was reclassified into six types: water (water bodies), woodland (tree cover and shrubland), grassland (herbaceous cover, grasslands, and lichens and mosses), cropland (agricultural crops), bareland (bare areas, sparse herbaceous cover, unconsolidated bare areas, and permanent snow and ice), and urban and built-up areas (urban areas and consolidated bare areas). The croplands north of the Amur were not included in the analysis for two reasons. First, there are few croplands in those regions because of an unsuitable climate and low human density. Second, small patches of croplands could scarcely be detected by the 300$\mathrm{m}$-resolution remote sensing devices, and the clear-cuts created by logging activities and forest fires, a widespread event in Siberian forests, can be misclassified as cropland.

\section{Identification of suitable wetland sites}

The selection of suitable wetland sites in the distribution ranges of each study species was achieved by calculating the probabilities of species occurrence in relation to environmental factors. We assumed that the migratory birds do not travel further north than their breeding ranges or further south than their non-breeding ranges. Therefore, for each species, we first selected all wetlands that fell within the study area (Appendix S2.1) as well as between their northernmost extent of the breeding range and southernmost extent of their non-breeding range ${ }^{95}$. Habitat selection by 
migratory waterfowl is mainly based on availability and suitability of wetlands and influenced by the type and extent of surrounding land-use types ${ }^{101}$. Therefore, we built a logistic regression model using the presence/absence of a study species in each wetland, in relation to lake area $\left(\mathrm{km}^{2}\right)$, elevation $(\mathrm{m}), x$ coordinates $(\mathrm{m}$; to represent the East-West gradient under the azimuthal equidistant projection) of lakes, and surrounding extent of suitable foraging areas, to predict the suitable wetland sites for each study species. Lakes with one or more observations of a study species were defined as presence records. We then randomly generated an equal number of absence records in the lakes where ebirder visited but without observations of the specific study species. Distances between roosting and foraging sites of waterfowl species in general do not exceed their maximum foraging flight distance ${ }^{102}$, so the surrounding extent of foraging areas was measured by the area $\left(\mathrm{km}^{2}\right)$ of grassland and cropland within a $32.5-\mathrm{km}$ radius buffer around each lake, which is the maximum mean foraging flight distances of ducks and geese ${ }^{103}$. Both $x$ coordinates, as measured by the center $x$ coordinate of each lake, and the squared $x$ coordinate, were added to the model because we assumed a dome-shaped relationship between the chance of a wetland being used by a specific species and the $x$-coordinate, for example, higher near the coast or higher in the center of their migration extent than at the edge.

Table 2.1 Landscape variables associated with waterfowl habitat degradation. All landscape metrics were measured in the suitable wetland sites in each $100 \times 100$ $\mathrm{km}$ grid cell. Wetland properties include water and surrounding grassland. The changes were quantified by change ratios from 1992 to 2012 .

\begin{tabular}{lll}
\hline Variable & Index for & Description \\
\hline Total Area (ha) & $\begin{array}{l}\text { Wetland } \\
\text { availability }\end{array}$ & Wetland size. \\
Mean Area (ha) & $\begin{array}{l}\text { Wetland } \\
\text { Fragmentation }\end{array}$ & The average wetland patch area. \\
$\begin{array}{l}\text { Proximity } \\
\text { Index }\end{array}$ & $\begin{array}{l}\text { Isolation } \\
\text { A measurement of relative isolation of the wetland patches. }\end{array}$ & $\begin{array}{l}\text { High value indicates habitat patches are connected to each } \\
\text { other within a buffer distance, while low proximity index } \\
\text { value indicates they are isolated from each other }{ }^{112} \text {. }\end{array}$ \\
$\begin{array}{l}\text { Total Water } \\
\text { Area (ha) }\end{array}$ & $\begin{array}{l}\text { Water area } \\
\text { Total }\end{array}$ & $\begin{array}{l}\text { A measurement of availability of water surface as roosting } \\
\text { habitats. }\end{array}$ \\
$\begin{array}{l}\text { Area (ha) } \\
\text { Total Crop } \\
\text { Area (ha) }\end{array}$ & availability & $\begin{array}{l}\text { A measurement of availability of grasslands as primary } \\
\text { food resources. }\end{array}$ \\
\hline
\end{tabular}


For each species, the best model with the smallest bias-adjusted Akaike's information criterion was selected ${ }^{104}$. By classifying the predicted probability of occurrence as presence or absence with a cutoff value of $50 \%$, the accuracy of the models was calculated by summing the number of true positive cases (classified by the model as presence and the species is present in reality) and true negatives (classified by the model as absence and the species is absent in reality) divided by total number of cases ${ }^{105}$. A wetland site was defined as suitable when the predicted probability of presence of the specific species exceeded 50\% (Appendix S2.4). The wetland area in subsequent analyses included these suitable lakes and a $32.5-\mathrm{km}$ buffer around each of these suitable lakes ${ }^{105}$.

All distances and coordinates were calculated under the azimuthal equidistant projection, and all areas were calculated under the cylindrical equal area. Calculations of the environmental factors were performed in ArcMap 10.2.1 (ESRI, San Diego, CA, USA). Logistic regressions were performed with package 'Ime4' 106, and model selections were performed with package 'MuMIn' 104 in R 3.3.3.

\section{Quantification of habitat degradation}

To quantify how habitats in these suitable wetland sites changed from 1992-2012, we calculated six landscape metrics including availability of water area, grassland, and wetland, and quantified wetland fragmentation and isolation, and changes in agriculture resources in 1992 and 2012, respectively (Table 2.1). Water and surrounding grasslands were aggregated into wetland properties, as both the area of open water and surrounding grasslands affect the suitability of a wetland for waterfowl 102,107. The size of a wetland is a key predictor for waterfowl species richness, and wetland connectivity and isolation are additional landscape metrics affecting waterfowl habitat quality 80,108 .

All landscape metrics were measured per suitable wetland site in each $100 \times 100 \mathrm{~km}$ grid cell, as the upper quartile of scales at which habitat configuration affects the distribution of species is approximately $100 \mathrm{~km}$, partly because the maximum radius of a species' foraging flight is generally smaller than $50 \mathrm{~km}$ 103,109-111. Water, grassland, and wetland availability were measured by the total area of water bodies, grassland, and wetlands, respectively. Wetland fragmentation was measured by the change in mean patch area of wetlands. Wetland isolation was quantified by the change in the proximity index, which equals the sum of the wetland patch area divided 
by the squared edge-to-edge distance between a wetland patch and the wetland patches whose edges are within $32.5 \mathrm{~km}$ around the specific patch 112 , as:

$$
\text { Proximity index }=\sum_{s=1}^{n} \frac{a_{i j s}}{d_{i j s}^{2}}
$$

where $n$ equals number of wetland patches within the suitable wetland sites in each $200 \times 200 \mathrm{~km}$ grid cell; $a_{i j s}$ is the area of wetland patch $i j$, which is within in a distance of $32.5 \mathrm{~km}$ around focal wetland patch $s$; $d_{i j s}$ is the edge-to-edge distance between wetland patch $i j$ and focal wetland patch $s$. The availability of agricultural resources was quantified by the total area of cropland.

All calculations were conducted under the azimuthal equidistant projection. Geographic data for calculating landscape metrics were prepared with ArcMap 10.2.1 (ESRI, San Diego, CA, USA). Fragstats 4.2113 was used to calculate landscape metrics.

\section{Exploration of species variation affected by habitat degradation}

Habitat degradation was quantified by the change ratios of the six landscape metrics from 1992 to 2012 in each $100 \times 100 \mathrm{~km}$ grid cell, as:

$$
\text { Change ratio }=\ln \left(\frac{V_{2012}}{V_{1992}}\right)
$$

Where $V_{1992}$ and $V_{2012}$ is the value of each landscape metric in 1992 and 2012, respectively. To better understand the latitudinal, national, and species-specific patterns of habitat degradation, the mean change ratio of each landscape metric in each 5-degree latitudinal zone (each zone is 5degree wide), each country, and in each breeding, non-breeding, and stopover area (the suitable wetland sites in between their breeding and nonbreeding ranges) of each study species was calculated by overlapping the species' ranges with the calculated six landscape metrics maps (grid cell: $100 \times 100 \mathrm{~km})$.

Three general linear models (GLMs) were applied to test 1) whether patterns of wetland degradation change over latitude and 2) whether the patterns differs among species with different migration extent (i.e., species with shorter and broader migration corridors versus those with longer and narrower migration corridors). The three dependent variables were the mean of absolute changes in the change ratios of wetland availability, 
fragmentation, and isolation, respectively. Independent variables of each model included one continuous variable (latitude) and one categorical variable (the species catalogue with two classes; i.e., ' 1 ' is species with longer and narrower migration corridors; ' 2 ' is species with shorter and broader migration corridors). We defined six out of the eight study species (tundra swan, bean goose, greater white-fronted goose, lesser white-fronted goose, common teal, and northern pintail) as species with longer and narrower migration corridors, with their seasonal distribution extending from the Lower Yangtze to Siberia. The swan goose and greylag goose were classified as species with shorter and broader migration corridors that breed in Mongolian regions and occupy more western parts of the flyway compared to the first group of species (Figure 2.1). This classification is in agreement with previous findings 56,92 .

The changes in landscape variables in different regions was calculated with ArcMap 10.2.1. The basic statistics were calculated in R 3.3.3, and the GLMs were carried out with package 'lme4' 106 in R 3.3.3.
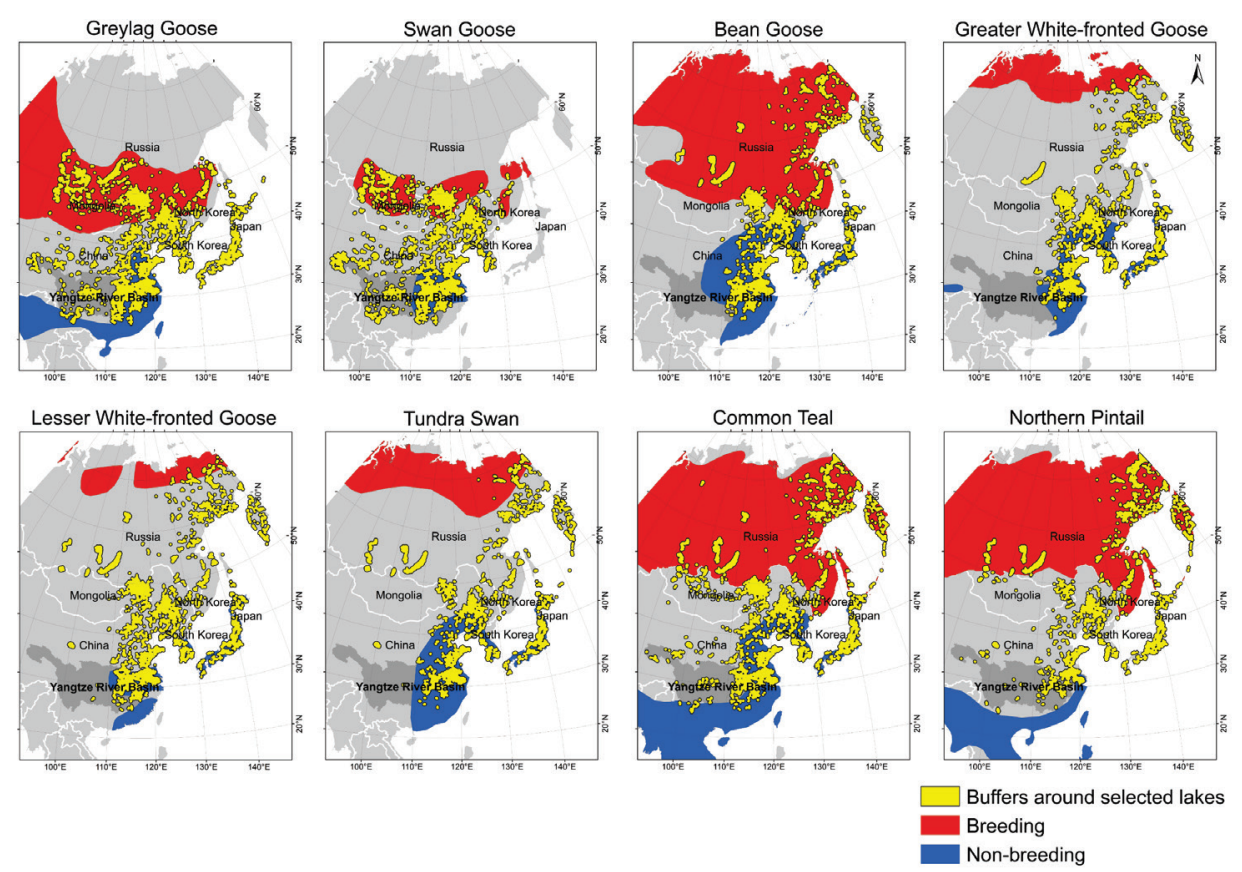

Figure 2.1 Suitable wetland sites (yellow polygons) for studied waterfowl species. The ranges of suitable wetland sites were used for subsequent analysis, and included suitable lakes and a $32.5-\mathrm{km}$ buffer around each of the suitable lakes. 


\subsection{Results}

\section{Environmental factors and the presence of waterfowl in wetland sites}

According to the best models, the presence of all goose and duck species was positively related to area of lakes, and the presence of all species (except lesser white-fronted goose) was positively related to surrounding food resources (i.e., grass and crop resources; Appendix 2.2). The probability of presence for greater white-fronted goose, lesser white-fronted goose, tundra swan, and northern pintail increased with decreasing elevation (Appendix 2.2).

\section{Habitat degradation in the flyway}

In the predicted suitable wetland sites (Figure 2.1), for all eight species, 4\% of the landscape was covered by water, and $26 \%$ and $21 \%$ of the landscape by grassland and cropland, respectively. The water area in suitable wetland sites in both non-breeding and breeding ranges of all eight species decreased during the 1992-2012 period, mainly in Southeast China, South Korea, Japan, Mongolia, and Northeast Russia (Figure2.2a).

As illustrated by the negative change in corresponding landscape variables in each grid, $43 \%, 51 \%$, and $45 \%$ of the landscape experienced wetland loss, fragmentation, and isolation, respectively (Figure 2.2). The three processes of wetland degradation happened simultaneously in $27 \%$ of the landscape, specifically in their non-breeding grounds in the Middle and Lower Yangtze River, Lower Yellow River, and Japan (Figure 2.2). Habitat availability improved in inland regions, including the Upper Yellow River, Korea, Mongolia, and Russia, which are important breeding grounds for the study species, as indicated by an increase in wetland area and a decrease in the level of wetland isolation (Appendix S2.7). 

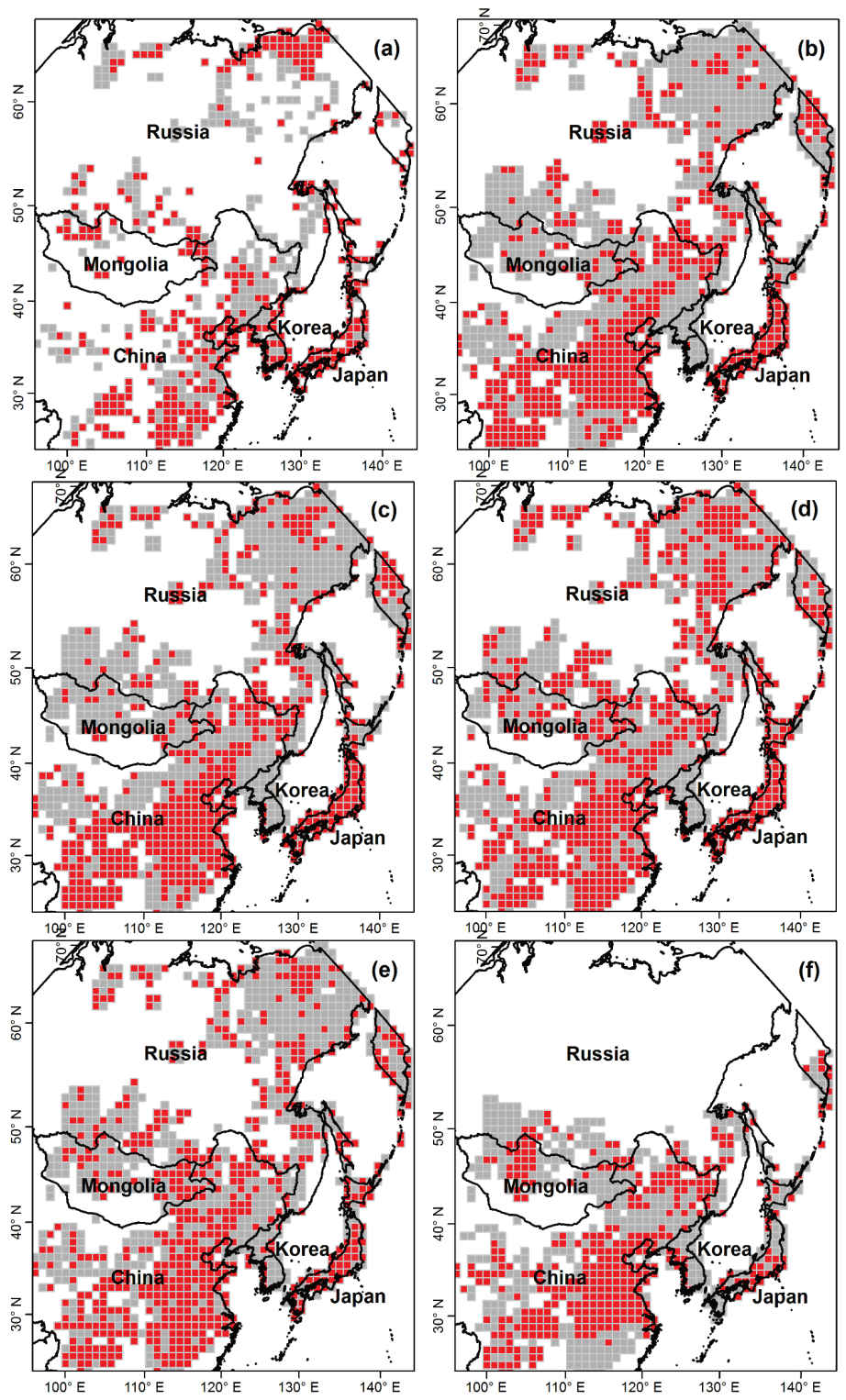

Changes from 1992 to 2012

Decrease $\quad$ Increase

Figure 2.2 Spatial patterns in changes in landscape metrics from 1992 to 2012. (a) Water loss as measured by the change in total water area; (b) Grassland loss as measured by the change in total grassland area; (c) Wetland availability as measured by the change in the total wetland area; (d) Wetland fragmentation as measured by the change in the mean patch area of wetlands; (e) Wetland isolation as indexed by the change in the proximity index of wetland patches; (f) Changes in agriculture resources as measured by the change in the total cropland area. A negative value indicates a decrease in corresponding landscape metrics. 


\section{Species-dependent effect of habitat degradation}

The eight species were all exposed to wetland loss, fragmentation, and isolation in their non-breeding grounds in China and Japan, but their breeding grounds improved in both Mongolia and Russia. Although the configuration of wetlands improved in the stopover areas of the bean goose, greater white-fronted goose, and tundra swan, that pass both China and Russia during migration, the other species were affected by wetland loss, fragmentation, and isolation in their stopover areas, especially for those species with stopover areas in China and Japan (Appendices S2.4 and S2.5). Generally, the migratory species were affected by habitat degradation in the southern part of their seasonal distributions, and their habitat availability improved in the northern part (Figure 2.3).

During 1992-2012, the wetland availability increased (or decreased less rapidly) with increasing latitude ( $\mathrm{GLM}, \beta=0.004, t=17.66, D F=4619, P<0.01$ ), and species with shorter and broader migration corridors had a significantly larger increase in wetland availability than species with longer and narrower migration corridors $(\beta=0.016, t=2.50, P=0.01)$. Similarly, the wetlands were less fragmented and isolated at higher latitude (GLM for wetland fragmentation, $\beta=0.007, t=17.64, D F=4619, P<0.01$; GLM for wetland isolation, $\beta=0.009, t=12.85, D F=4619, P<0.01$ ), and species with shorter and broader migration corridors had significantly less habitat fragmentation and isolation than species with longer and narrower migration corridors $(\beta=0.088, t=8.31, P<0.01 ; \beta=0.049, t=2.77, P=0.01)$. Although wetland area for species with longer and narrower migration corridors increased at higher latitudes, that for species with shorter and broader migration corridors and a more western distribution increased more between $30 \mathrm{~N}-50 \mathrm{~N}$ in the areas of the Upper Yellow River and Mongolia (Figure 2.3). 

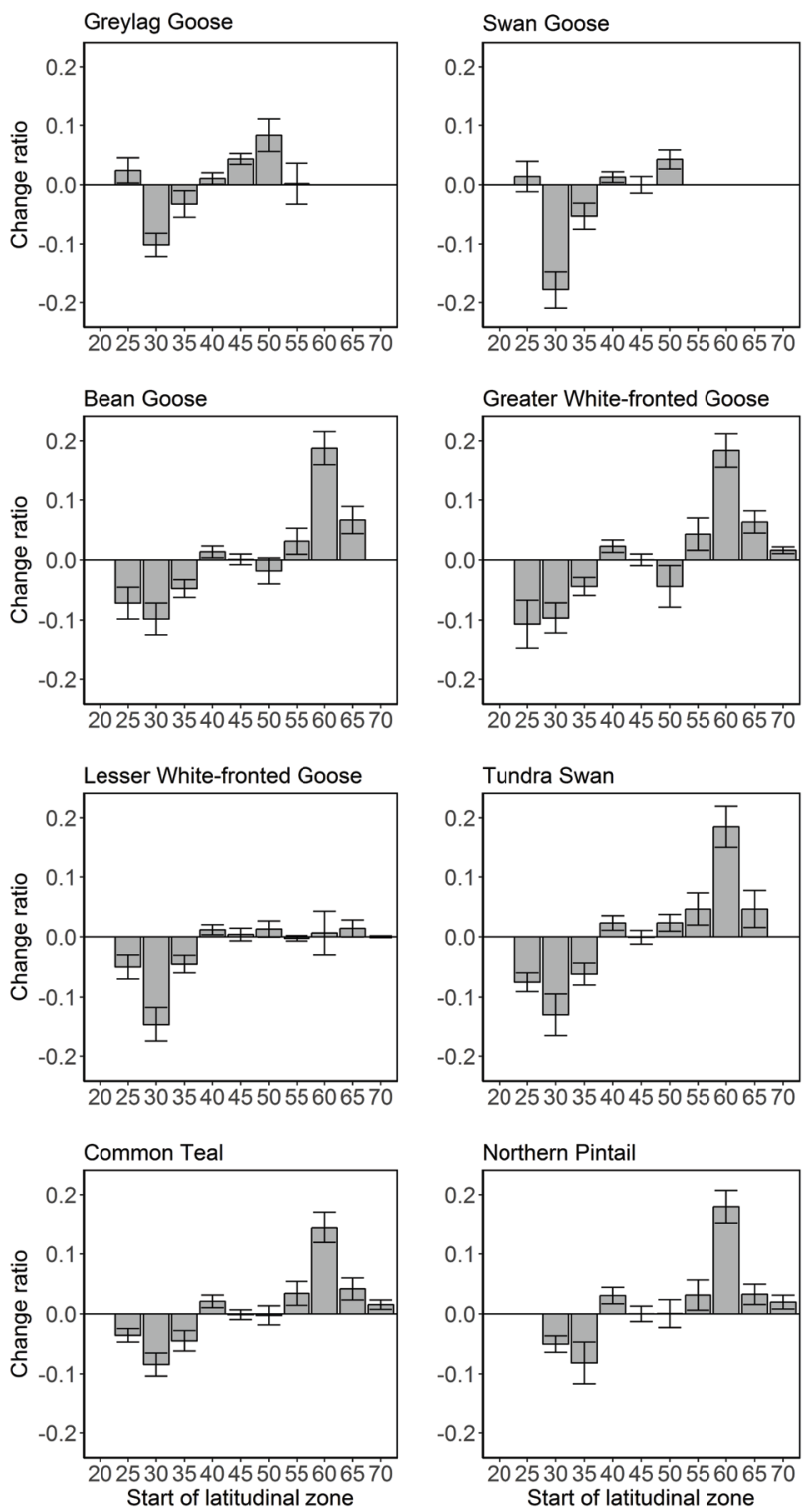

Figure 2.3 Latitudinal patterns of change ratio (mean \pm standard deviation) of wetland availability (water surface and surrounding grasslands) in the suitable wetland sites from 1992-2012; x-axis represents five-degree latitudinal zones. A negative value indicates a decrease in area of wetlands in the corresponding latitudinal zone while a positive value indicates an increase. 


\subsection{Discussion}

The seasonal distributions of migratory waterfowl species determine the extent to which they are exposed to habitat degradation, which varies from place to place. As for migratory waterfowl in the East Asian-Australasian Flyway, habitat availability simultaneously degraded in the southeastern part of the flyway, i.e., in the coastal regions in China and Japan, but improved in inland regions of the western part of flyway (Figure 2.2). Species with longer and narrower migration corridors that concentrate their migrations along the eastern coast could benefit less from improved habitat conditions in the southern part of their migration flyway compared to those with shorter and broader migration corridors. However, species with longer and narrower migration corridors could reach improved habitat conditions in Russia, in the northern part of their distribution range.

Areas of grassland and wetland in the suitable wetland sites in southern and eastern China and Japan decreased from 1992 to 2012 (Figure 2.2b and c), and those areas could become spatial bottlenecks for species with main stopovers in these regions. Migratory species with spatial bottlenecks in degraded regions could be less resilient to habitat changes because of limited alternatives ${ }^{114,115}$. These species must either skip the degraded wetlands or accept suboptimal conditions ${ }^{93}$, leading to increased costs of migration, and consequently increased mortality during migration, and probably reduced efficiency of energy intake and reproduction. It could be difficult for the species experiencing successive habitat loss while migrating from their non-breeding to breeding grounds to replenish energy stores and maintain optimal body reserves for reproduction.

By investigating patterns of habitat change at the flyway scale, we further highlight the relationship between migratory extent and speciesspecific effects of environmental changes. Previous studies have found that migratory extent can affect species resilience to environmental changes. Species with a longer migration distance 74,92 , a smaller non-breeding area compared to breeding area ${ }^{56}$, and a larger reliance on specific regions (e.g., South America and Yellow Sea tidal mudflat) are more vulnerable compared to others 35,116 . These facts can be explained when we relate their distributions to spatial patterns of habitat degradation at a flyway scale. Species occupying broader extent with more parallel alternative sites have plasticity in their reaction to habitat degradation. Species with shorter and broader migration corridors migrate across both degraded landscapes in the 
east and areas that have increased habitat availability in the west of the flyway. Despite the shorter migration distance of these species compared to the other study species, their migratory dispersion (i.e., larger non-breeding range size relative to breeding) influences their resilience to habitat degradation. GPS tracking data also have shown that the swan geese from Mongolia migrate over a broad front, using a parallel configuration of stopover sites, although these geese share the same non-breeding and breeding grounds 117 . Swan geese can use stopover sites located at the western part of their flyway, where habitat degradation of stopover sites is lower than in the eastern part.

The wetlands of the East Asian-Australasian Flyway have been threatened by habitat loss, fragmentation, and isolation over the past two decades, which can subsequently impact migratory waterfowl by depleting resources and isolating wetland sites. Wetland degradation in eastern China and Japan contributed most to habitat destruction in the flyway from 19922012, partly as a consequence of rapid urbanization and socioeconomic development in East Asian countries since $1992{ }^{118}$. Wetlands on their nonbreeding grounds with intensive human activities lost much of their area, triggering a human-wildlife conflict in which birds and people compete for resources 119,120 . China has made rapid economic development since the economic reform in 1978, which is accompanied by accelerating environmental degradation, e.g., decreasing wetland area ${ }^{121}$. Agricultural expansion is one of the most important threats to wetlands by forms of wetland conversion or water drainage for irrigation 77,121 , and pollution and insufficient funding for protection are other contributing factors to wetland degradation 121 . The coastal regions are confronted with larger problems compared to inland areas because of increased human pressure and sealevel rise, e.g., a considerable part of Japanese wetlands is threatened $73,88,122$. On the contrary, natural habitats have recovered in the temperate zones of Russia due to a low human density and a widespread land abandonment since the sweeping reorganization of the Russian agriculture in 1990 s 123 .

Natural grasslands, as the primary foraging areas for waterfowl, are vulnerable because they are more sensitive to climate change than most human land-use types ${ }^{124}$. Agriculture expanded around most wetlands and increased food resources for waterfowl, according to our modelling. For example, some wetlands in southeastern China are efficiently cultivated with 
multiple rice farming systems ${ }^{125}$. However, these benefits might be a tradeoff against greater human disturbance around these wetlands and increased wetland loss to land reclamation. Farmlands reclaimed in or around lakes and wetlands, sacrifice roosting and primary foraging sites (e.g., recessional grasslands) of waterfowl. Thus, waterfowl species are also more confined to their natural habitats instead of exploiting surrounding farmlands in their non-breeding grounds, and they tend to select habitats with lower human pressures in China 51,126. Moreover, there are other forms of habitat degradation for migratory waterfowl which have not been measured by the metrics quantified in our study, but can decrease waterfowl species diversity and reduce wetland quality, e.g., pollution with pesticides and heavy metals, changes in water levels by dams, illegal killing and hunting activities, and low efficiency of local conservation regulations 127,128 . In the future, ecological restoration projects considering these factors might offer some potential 129,130 to conserve critical wetlands in the Middle and Lower Yangtze River, Lower Yellow River, and Japan.

Wetland degradation poses severe challenges to migratory species because wetland loss can reduce local abundance and species richness ${ }^{79}$. Considering each wetland patch as an island surrounded by suboptimal or unsuitable habitats, both the loss of wetland area and isolation from other wetlands can trigger local extinction of populations 131,132 . The vulnerability of a population increases when even only a part of the migration network across a large spatial extent is affected ${ }^{73}$. The population decline of migratory birds in relation to habitat degradation in the East AsianAustralasian Flyway has therefore triggered concern 55,87,133, as East Asian populations of bean goose, greater white-fronted goose, lesser white-fronted goose, common teal, swan goose, and northern pintail are generally decreasing 55,87,134. Previous studies have suggested that a couple of bottleneck sites in their migration network explain these population declines. For example, the Yellow Sea tidal mudflat has shrunk by more than $65 \%$, and consequently, the migratory shorebirds that highly rely on the Yellow Sea tidal mudflat experienced large population declines ${ }^{35}$. The effect of habitat degradation on population size, especially for those species that use multiple stopover sites, depends not only on the overall extent of habitat degradation 73,135 , but also on where this degradation occurs ${ }^{94}$. Our results demonstrate that habitat degradation in the migration flyway has a strong spatial component, which may explain differences in the population dynamics of migratory waterfowl species. 
Because migratory species might be able to respond to habitat degradation by altering migration routes, future studies should focus on both specific regions and on the integrity of the whole migration network and on the plasticity of the species in terms of migratory movements and visited stopover sites. Hence, a network approach is required to better understand changes in migration strategy and population dynamics of migratory species. Remote-sensing techniques and temporal land cover data allow us to monitor the environmental changes at flyway scale ${ }^{136}$. There is, therefore, a demand for higher-accuracy and finer-resolution land cover datasets to support studies on the large-scale environmental changes in the framework of migration and conservation biology.

\subsection{Conclusion}

This study relates species seasonal distribution to species-dependent effects of habitat degradation in the migratory flyway. We have demonstrated that eight waterfowl species in the East Asian-Australasian Flyway are all exposed to habitat degradation in their non-breeding areas, but that conditions around wetland sites improve with increasing latitudes. Comparing changes at the same latitude, wetland sites for species with longer and narrower migration corridors degraded more from 1992 to 2012 than for species with shorter and broader migration. We conclude that migratory species with narrower distributions and longer migration distances are exposed to a higher level of habitat degradation because they have fewer parallel sites to provide alternative stopover, roosting, or foraging sites when habitat is degraded or lost. Hence, selection of important conservation regions for migratory birds should not only depend on local conditions of wetland sites but also take species-specific seasonal distributions into account. Especially, more efforts should be targeted along the migration routes of species with a narrow seasonal distribution and spatial bottlenecks in degraded regions of the flyway. Moreover, it is necessary to limit reclamation of wetland resources and unrestrained water drainage in regions of the East Asian-Australasian Flyway because wetlands in the Middle and Lower Yangtze River, Lower Yellow River, and Japan are major non-breeding grounds as well as important stopover areas for many waterbird species. 


\subsection{Acknowledgements}

We thank Yingying Wang (Wageningen University, the Netherlands) for help with the statistical analyses. We thank Zezhong Wang (Peking University, China), Zhouyuan Li (Wageningen University, the Netherlands), and Jing Li (Wageningen University, the Netherlands) for their suggestions on spatial scales and quantifications of habitat changes by landscape metrics. We thank Dorit Gross (Wageningen University, the Netherlands) for her suggestions on land cover products. Financial support was provided by the National Key R\&D Program of China (No. 2017YFA0604404) and the National Natural Science Foundation of China (No. 41471347) and Chinese Scholarship Council (No. 201600090128). 


\section{Supplementary information}

Appendix S2.1 A map of the study area from the Yangtze River Basin. Regions in China, Mongolia, North Korea, South Korea, Japan, and Russia intersect the area. The major wetland sites were identified from the Global Lakes and Wetlands Database (worldwildlife.org).

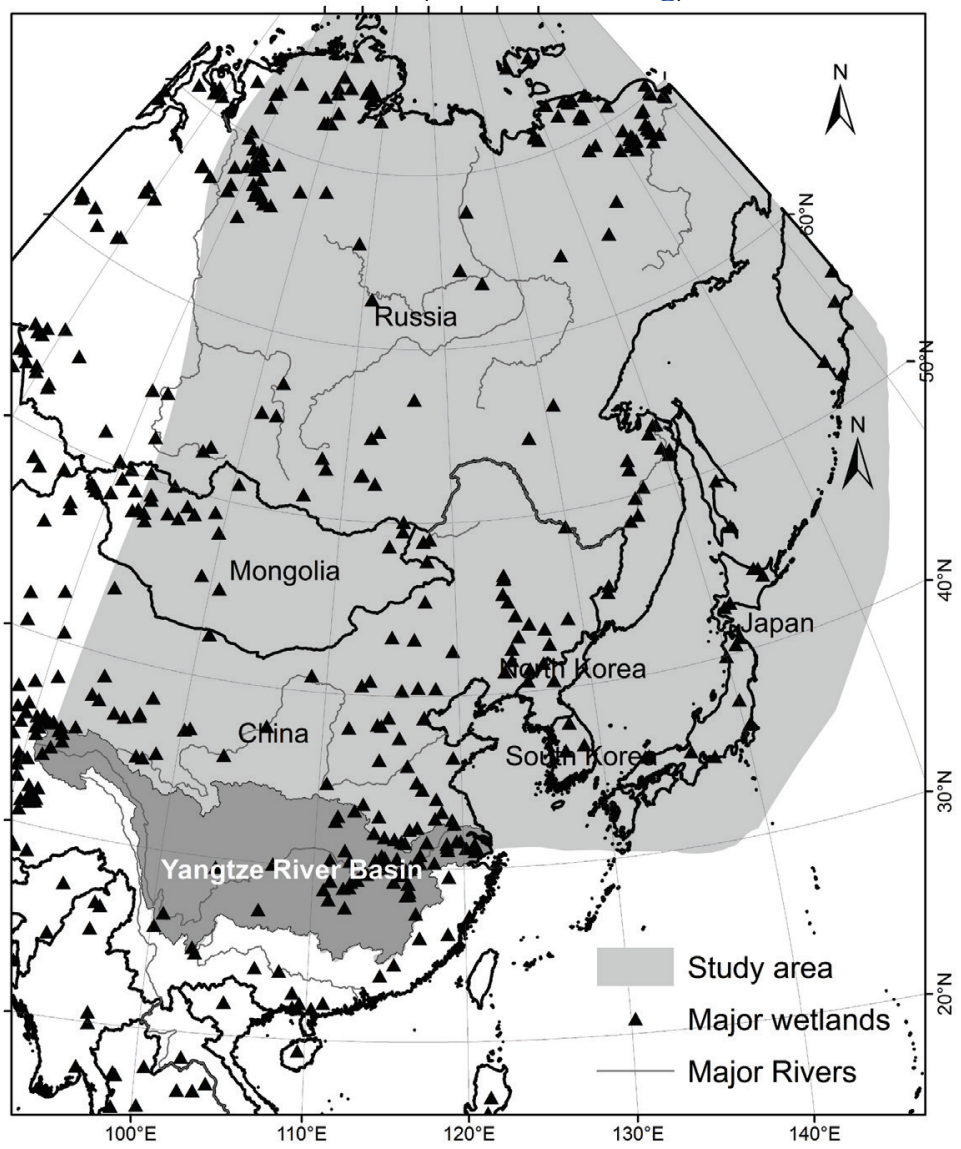


Appendix S2.2 Logistic regression models of environmental factors on species presence. All models with a difference of $\leq 4$ in AIC are listed. Best models with the lowest AIC were used for the prediction of species presence and absence.

\begin{tabular}{|c|c|}
\hline Model & AIC \\
\hline \multicolumn{2}{|l|}{ Greylag Goose } \\
\hline Lake Area $\left[\log \left(\mathrm{km}^{2}\right)\right]+$ Grass\&Crop Resources $\left[\log \left(\mathrm{km}^{2}\right)\right]+x$ & 162.2 \\
\hline Lake Area $\left[\log \left(\mathrm{km}^{2}\right)\right]+x$ & 163.9 \\
\hline Lake Area $\left[\log \left(\mathrm{km}^{2}\right)\right]+$ Elevation $[\log (\mathrm{m})]+$ Grass\&Crop Resources & 164.0 \\
\hline \multicolumn{2}{|l|}{$\left[\log \left(\mathrm{km}^{2}\right)\right]+x$} \\
\hline Lake Area $\left[\log \left(\mathrm{km}^{2}\right)\right]+$ Grass\&Crop Resources $\left[\log \left(\mathrm{km}^{2}\right)\right]+x+x^{2}$ & 164.0 \\
\hline Lake Area $\left[\log \left(\mathrm{km}^{2}\right)\right]+$ Elevation $[\log (\mathrm{m})]+x$ & 164.7 \\
\hline Lake Area $\left[\log \left(\mathrm{km}^{2}\right)\right]+x+x^{2}$ & 164.7 \\
\hline Lake Area $\left[\log \left(\mathrm{km}^{2}\right)\right]+$ Elevation $[\log (\mathrm{m})]+$ Grass\&Crop Resources & 165.8 \\
\hline \multicolumn{2}{|l|}{$\left[\log \left(\mathrm{km}^{2}\right)\right]+x+x^{2}$} \\
\hline Lake Area $\left[\log \left(\mathrm{km}^{2}\right)\right]+$ Elevation $[\log (\mathrm{m})]+x+x^{2}$ & 166.2 \\
\hline \multicolumn{2}{|l|}{ Swan Goose } \\
\hline Lake Area $\left[\log \left(\mathrm{km}^{2}\right)\right]+$ Grass\&Crop Resources $[\log (\mathrm{km} 2)]+x+x^{2}$ & 438.4 \\
\hline Lake Area $\left[\log \left(\mathrm{km}^{2}\right)\right]+$ Elevation $[\log (\mathrm{m})]+$ Grass\&Crop Resources & 440.6 \\
\hline \multicolumn{2}{|l|}{$\left[\log \left(\mathrm{km}^{2}\right)\right]+x+x^{2}$} \\
\hline \multicolumn{2}{|l|}{ Bean Goose } \\
\hline Lake Area $\left[\log \left(\mathrm{km}^{2}\right)\right]+$ Grass\&Crop Resources $\left[\log \left(\mathrm{km}^{2}\right)\right]+x+x^{2}$ & 400.7 \\
\hline Lake Area $\left[\log \left(\mathrm{km}^{2}\right)\right]+$ Elevation $[\log (\mathrm{m})]+$ Grass $\&$ Crop Resources & 401.7 \\
\hline \multicolumn{2}{|l|}{$\left[\log \left(\mathrm{km}^{2}\right)\right]+x+x^{2}$} \\
\hline Grass\&Crop Resources $\left[\log \left(\mathrm{km}^{2}\right)\right]+x+x^{2}$ & 404.0 \\
\hline \multicolumn{2}{|l|}{ Greater White-fronted Goose } \\
\hline Lake Area $\left[\log \left(\mathrm{km}^{2}\right)\right]+$ Elevation $[\log (\mathrm{m})]+$ Grass\&Crop Resources & 733.0 \\
\hline \multicolumn{2}{|l|}{$\left[\log \left(\mathrm{km}^{2}\right)\right]+x+x^{2}$} \\
\hline Lake Area $\left[\log \left(\mathrm{km}^{2}\right)\right]+$ Grass $\&$ Crop Resources $\left[\log \left(\mathrm{km}^{2}\right)\right]+x+x^{2}$ & 734.2 \\
\hline \multicolumn{2}{|l|}{ Lesser White-fronted Goose } \\
\hline Lake Area $\left[\log \left(\mathrm{km}^{2}\right)\right]+$ Elevation $[\log (\mathrm{m})]+x$ & 126.2 \\
\hline Lake Area $\left[\log \left(\mathrm{km}^{2}\right)\right]+$ Elevation $[\log (\mathrm{m})]$ & 127.7 \\
\hline Lake Area $\left[\log \left(\mathrm{km}^{2}\right)\right]+$ Elevation $[\log (\mathrm{m})]+$ Grass\&Crop Resources $\left[\log \left(\mathrm{km}^{2}\right)\right]+x$ & 128.6 \\
\hline Lake Area $\left[\log \left(\mathrm{km}^{2}\right)\right]+$ Elevation $[\log (\mathrm{m})]+x+x^{2}$ & 128.7 \\
\hline Lake Area $\left[\log \left(\mathrm{km}^{2}\right)\right]+$ Elevation $[\log (\mathrm{m})]+$ Grass\&Crop Resources $\left[\log \left(\mathrm{km}^{2}\right)\right]$ & 129.3 \\
\hline Lake Area $\left[\log \left(\mathrm{km}^{2}\right)\right]+$ Elevation $[\log (\mathrm{m})]+x^{2}$ & 129.7 \\
\hline \multicolumn{2}{|l|}{ Tundra Swan } \\
\hline Elevation $[\log (\mathrm{m})]+$ Grass\&Crop Resources $\left[\log \left(\mathrm{km}^{2}\right)\right]+x$ & 440.1 \\
\hline Lake Area $\left[\log \left(\mathrm{km}^{2}\right)\right]+$ Elevation $[\log (\mathrm{m})]+$ Grass\&Crop Resources $\left[\log \left(\mathrm{km}^{2}\right)\right]+x$ & 440.6 \\
\hline Elevation $[\log (\mathrm{m})]+$ Grass\&Crop Resources $\left[\log \left(\mathrm{km}^{2}\right)\right]+x+x^{2}$ & 441.8 \\
\hline Lake Area $\left[\log \left(\mathrm{km}^{2}\right)\right]+$ Elevation $[\log (\mathrm{m})]+$ Grass\&Crop Resources & 442.1 \\
\hline \multicolumn{2}{|l|}{$\left[\log \left(\mathrm{km}^{2}\right)\right]+x+x^{2}$} \\
\hline \multicolumn{2}{|l|}{ Common Teal } \\
\hline Lake Area $\left[\log \left(\mathrm{km}^{2}\right)\right]+$ Grass\&Crop Resources $\left[\log \left(\mathrm{km}^{2}\right)\right]+x+x^{2}$ & 893.0 \\
\hline Lake Area $\left[\log \left(\mathrm{km}^{2}\right)\right]+$ Grass\&Crop Resources $\left[\log \left(\mathrm{km}^{2}\right)\right]+x$ & 894.0 \\
\hline Lake Area $\left[\log \left(\mathrm{km}^{2}\right)\right]+$ Elevation $[\log (\mathrm{m})]+$ Grass\&Crop Resources & 895.0 \\
\hline$\left[\log \left(\mathrm{km}^{2}\right)\right]+x+x^{2}$ & \\
\hline $\begin{array}{l}\text { Lake Area }\left[\log \left(\mathrm{km}^{2}\right)\right]+\text { Elevation }[\log (\mathrm{m})]+\text { Grass\&Crop Resources } \\
{\left[\log \left(\mathrm{km}^{2}\right)\right]+x+x^{2}}\end{array}$ & 896.1 \\
\hline
\end{tabular}


(Continued Appendix S2.2)

\begin{tabular}{lc}
\hline Model & AIC \\
\hline Northern Pintail & 1873.4 \\
$\quad$ Lake Area $\left[\log \left(\mathrm{km}^{2}\right)\right]+$ Elevation $[\log (\mathrm{m})]+$ Grass\&Crop Resources & \\
$\quad\left[\log \left(\mathrm{km}^{2}\right)\right]+x+x^{2}$ & 1874.3 \\
$\quad$ Lake Area $\left[\log \left(\mathrm{km}^{2}\right)\right]+$ Elevation $[\log (\mathrm{m})]+$ Grass\&Crop Resources & \\
{$\left[\log \left(\mathrm{km}^{2}\right)\right]+x$} & \\
\hline
\end{tabular}

Appendix S2.3 Number of wetland sites in total (all) and selected as suitable sites (selected) per species. The two catalogue groups of species were defined by their distribution. Species classified as Catalogue 1 are the ones with shorter and broader flyways while those as Catalogue 2 are the ones with longer and narrower flyways.

\begin{tabular}{ccccc}
\hline Catalogue & Species & All & Selected & $\begin{array}{c}\text { Proportion } \\
\text { of selected }\end{array}$ \\
\hline 1 & Greylag goose & 6592 & 1527 & $23.2 \%$ \\
1 & Swan goose & 5951 & 2657 & $44.6 \%$ \\
2 & Bean goose & 25511 & 2925 & $11.5 \%$ \\
2 & Greater white-fronted & 25511 & 3045 & $11.9 \%$ \\
& goose & & & \\
2 & Lesser white-fronted & 22693 & 11180 & $49.3 \%$ \\
& goose & & & \\
2 & Tundra swan & 25234 & 1664 & $6.6 \%$ \\
2 & Common teal & 22631 & 5548 & $24.5 \%$ \\
\hline & Northern Pintail & 25042 & 5006 & $20.0 \%$ \\
\hline
\end{tabular}


Appendix S2.4 Results of the logistic regressions of environmental factors on species presence, showing the performance of the best models and regression coefficients for environmental factors included in the best models. $\triangle$ AIC is the difference between the AIC of the best model and the second-best model. Grass and Crop Resources were measured by the area of grasslands and croplands within the $32.5-\mathrm{km}$ buffer surrounding each lake; $\mathrm{x}=$ centre $\mathrm{x}$ coordinate of each lake.

\begin{tabular}{|c|c|c|c|c|}
\hline Model & Coefficient & $\begin{array}{l}\text { Standard } \\
\text { Error }\end{array}$ & $z$-value & $\mathrm{p}$-value \\
\hline \multicolumn{5}{|c|}{ Greylag Goose $(\mathrm{N}=178, \mathrm{AIC}=162.2, \Delta \mathrm{AIC}=1.7$, accuracy $=76.4 \%)$} \\
\hline (Intercept) & -3.385 & 1.325 & -2.556 & $0.011 *$ \\
\hline Lake Area $\left[\log \left(\mathrm{km}^{2}\right)\right]$ & 1.137 & 0.252 & 4.516 & $<0.001 * * *$ \\
\hline Grass\&Crop Resources $\left[\log \left(\mathrm{km}^{2}\right)\right]$ & 0.520 & 0.407 & 1.276 & 0.202 \\
\hline$x$ & -0.001 & 0.0002 & -5.088 & $<0.001 * * *$ \\
\hline \multicolumn{5}{|c|}{ Swan Goose $(\mathrm{N}=114, \mathrm{AIC}=438.4, \Delta \mathrm{AIC}=2.2$, accuracy $=72.8 \%)$} \\
\hline (Intercept) & -1.216 & 0.656 & -1.854 & $0.064^{-}$ \\
\hline Lake Area $\left[\log \left(\mathrm{km}^{2}\right)\right]$ & 0.822 & 0.146 & 5.628 & $<0.001 * * *$ \\
\hline Grass\&Crop Resources $\left[\log \left(\mathrm{km}^{2}\right)\right]$ & 0.399 & 0.198 & 2.022 & $0.043 *$ \\
\hline$X$ & $-3.628 \mathrm{e}-04$ & $1.348 \mathrm{e}-04$ & -2.692 & $0.007 * *$ \\
\hline$x^{2}$ & $-6.455 e-07$ & $1.159 \mathrm{e}-07$ & -5.570 & $<0.001 * * *$ \\
\hline \multicolumn{5}{|c|}{ Bean Goose $(\mathrm{N}=394, \mathrm{AIC}=400.7, \Delta \mathrm{AIC}=1.0$, accuracy $=68.5 \%)$} \\
\hline (Intercept) & -3.841 & 1.108 & -3.467 & $<0.001 * * *$ \\
\hline Lake Area $\left[\log \left(\mathrm{km}^{2}\right)\right]$ & 0.374 & 0.369 & 2.222 & 0.026 * \\
\hline Grass\&Crop Resources $\left[\log \left(\mathrm{km}^{2}\right)\right]$ & 1.280 & 0.351 & 3.650 & $<0.001 * * *$ \\
\hline$x$ & $1.472 \mathrm{e}-03$ & $2.638 \mathrm{e}-04$ & 5.579 & $<0.001 * * *$ \\
\hline$x^{2}$ & $-7.548 \mathrm{e}-07$ & $1.715 \mathrm{e}-07$ & -4.401 & $<0.001 * * *$ \\
\hline \multicolumn{5}{|c|}{ Greater White-fronted Goose $(\mathrm{N}=714, \mathrm{AIC}=733.0, \Delta \mathrm{AIC}=1.2$, accuracy $=78.4 \%)$} \\
\hline (Intercept) & -3.528 & 1.143 & -3.088 & $0.002 * *$ \\
\hline Lake Area $\left[\log \left(\mathrm{km}^{2}\right)\right]$ & 0.476 & 0.137 & 3.485 & $<0.001 * * *$ \\
\hline Elevation $[\log (\mathrm{m})]$ & -0.302 & 0.172 & -1.756 & $0.079^{-}$ \\
\hline Grass\&Crop Resources $\left[\log \left(\mathrm{km}^{2}\right)\right]$ & 1.383 & 0.333 & 4.153 & $<0.001 * * *$ \\
\hline$x$ & $2.672 \mathrm{e}-03$ & $3.004 \mathrm{e}-04$ & 8.895 & $<0.001 * * *$ \\
\hline$x^{2}$ & $-1.513 e-06$ & $1.814 \mathrm{e}-07$ & -8.342 & $<0.001 * * *$ \\
\hline \multicolumn{5}{|c|}{ Lesser White-fronted Goose $(\mathrm{N}=114, \mathrm{AIC}=126.2, \Delta \mathrm{AIC}=1.5$, accuracy $=72.8 \%)$} \\
\hline (Intercept) & 3.116 & 0.978 & 3.187 & $0.001 * *$ \\
\hline Lake Area $\left[\log \left(\mathrm{km}^{2}\right)\right]$ & 0.780 & 0.294 & 2.655 & $0.008 * *$ \\
\hline Elevation $[\log (\mathrm{m})]$ & -1.821 & 0.460 & -3.959 & $<0.001 * * *$ \\
\hline$x$ & $4.268 \mathrm{e}-04$ & $2.434 \mathrm{e}-04$ & 1.753 & $0.080^{-}$ \\
\hline \multicolumn{5}{|c|}{ Common Teal $(\mathrm{N}=816, \mathrm{AIC}=893.0, \Delta \mathrm{AIC}=1.0$, accuracy $=75.5 \%)$} \\
\hline (Intercept) & 1.979 & 0.614 & -3.222 & $0.001 * *$ \\
\hline Lake Area $\left[\log \left(\mathrm{km}^{2}\right)\right]$ & .562 & 0.103 & 5.446 & $<0.001 * * *$ \\
\hline $\begin{array}{l}\text { Grass\&Crop Resources } \\
{\left[\log \left(\mathrm{km}^{2}\right)\right]}\end{array}$ & .470 & 0.194 & 2.425 & $0.015 *$ \\
\hline$x$ & $.187 \mathrm{e}-03$ & $1.075 \mathrm{e}-04$ & 11.046 & $<0.001 * * *$ \\
\hline$x^{2}$ & $1.315 \mathrm{e}-07$ & $7.755 \mathrm{e}-08$ & -1.695 & $0.090^{-}$ \\
\hline \multicolumn{5}{|c|}{ Northern Pintail $(\mathrm{N}=2186, \mathrm{AIC}=1873.4, \Delta \mathrm{AIC}=0.9$, accuracy $=81.0 \%)$} \\
\hline (Intercept) & 2.704 & 0.558 & -4.846 & $<0.001 * * *$ \\
\hline Lake Area $\left[\log \left(\mathrm{km}^{2}\right)\right]$ & 298 & 0.079 & 3.748 & $<0.001 * * *$ \\
\hline Elevation $[\log (\mathrm{m})]$ & 0.253 & 0.096 & -2.640 & $0.008 * *$ \\
\hline Grass\&Crop Resources & .647 & 0.154 & 4.211 & $<0.001 * * *$ \\
\hline$\left[\log \left(\mathrm{km}^{2}\right)\right]$ & & & & \\
\hline$x$ & $.669 \mathrm{e}-03$ & $1.044 \mathrm{e}-04$ & 15.997 & $<0.001 * * *$ \\
\hline$x^{2}$ & $.252 \mathrm{e}-07$ & $7.163 \mathrm{e}-08$ & 1.748 & $0.080^{-}$ \\
\hline \multicolumn{5}{|c|}{ Tundra Swan $(\mathrm{N}=446, \mathrm{AIC}=440.1, \Delta \mathrm{AIC}=0.5$, accuracy $=78.3 \%)$} \\
\hline (Intercept) & -6.468 & 1.473 & -4.391 & $<0.001 * * *$ \\
\hline Elevation $[\log (\mathrm{m})]$ & -0.660 & 0.185 & -3.573 & $<0.001 * * *$ \\
\hline Grass\&Crop Resources $\left[\log \left(\mathrm{km}^{2}\right)\right]$ & 2.231 & 0.417 & 5.350 & $<0.001 * * *$ \\
\hline$x$ & $1.741 \mathrm{e}-03$ & $1.934 \mathrm{e}-04$ & 9.008 & $<0.001 * * *$ \\
\hline
\end{tabular}





\section{Loss of functional connectivity in migration networks induces population decline in migratory birds}

Xu Y., Si Y., Wang Y., Zhang Y., Prins H. H. T., Cao L., de Boer W. F. Loss of functional connectivity in migration networks induces population decline in migratory birds. Ecological Applications 29: e01960 (2019) 


\section{Abstract}

Migratory birds rely on a habitat network along their migration routes by temporarily occupying stopover sites between breeding and non-breeding grounds. Removal or degradation of stopover sites in a network might impede movement, and thereby reduce migration success and survival. The extent to which the breakdown of migration networks, due to changes in land use, impacts the population sizes of migratory birds is poorly understood. We measured the functional connectivity of migration networks of waterfowl species that migrate over the East Asian-Australasian Flyway from 1992-2015. We analysed the relationship between changes in nonbreeding population sizes and changes in functional connectivity, while taking into account other commonly-considered species traits, using a Phylogenetic Linear Mixed Model. We found that population sizes significantly declined with a reduction in the functional connectivity of migration networks; no other variables were important. We conclude that the current decrease in functional connectivity, due to habitat loss and degradation in migration networks, can negatively and crucially impact population sizes of migratory birds. Our findings provide new insights into the underlying mechanisms that affect population trends of migratory birds under environmental changes. Establishment of international agreements leading to the creation of systematic conservation networks associated with migratory species' distributions and stopover sites may safeguard migratory bird populations. 


\subsection{Introduction}

Recent trends in habitat loss and degradation strongly impact the survival and reproduction of wildlife species $35,137,138$. Habitat loss and degradation reduce functional connectivity, i.e., the degree to which landscape elements promote animal movements within and between habitat patches 139-141. Functional connectivity links landscape features with species' dispersal traits and is critical for understanding how the spatial distribution of suitable landscapes may influence populations of migratory species. The functional connectivity of migration networks is defined here as the degree to which the habitat configuration facilitates bird movements both within and between the breeding, non-breeding, and stopover sites.

Many migratory birds rely on a network of habitat patches as they travel between breeding and non-breeding areas. For example, many birds take advantage of multiple stopover sites during their migration for resting or refuelling before migrating further and breeding 36,67,89. Hence, connectivity among sites along migration flyways is essential for their survival and reproduction ${ }^{44}$ and thereby can play a vital role in the population dynamics of these species. A stable network of stopover sites is an important component for maintaining stable or increasing populations of migratory species ${ }^{142}$. In contrast, loss of habitat and loss of network connectivity may negatively impact migratory bird populations 73 .

The degree to which losses in the functional connectivity of a migration network, as a consequence of habitat loss and degradation, contribute to population declines in migratory birds remains unknown. So far, the relationships between functional connectivity of migration networks and population trends has not been investigated empirically ${ }^{35}$. One reason for this is the challenge of quantifying the connectivity of habitat networks at a flyway scale over extended periods of time, though such quantification at these relatively large spatial and temporal scales is essential when the goal is to assess links between functional connectivity and population dynamics of migratory species.

We hypothesize that functional connectivity as well as other previously studied predictors together drive population trends of migratory birds, so that population sizes of migratory birds decrease with a decreasing functional connectivity of their migration networks. Species, such as those with longer migration distances ${ }^{92}$, with a smaller size of their non-breeding ranges relative to breeding ranges (migratory dispersion) ${ }^{56}$, and with smaller 
breeding ranges ${ }^{143}$, are more likely to experience population declines. Others, such as species with a relatively small body mass ${ }^{88}$, large clutch size ${ }^{144}$ and short generation length ${ }^{143}$, are less likely to decline.

To test this hypothesis, we measured the functional connectivity of migration networks of eight waterfowl species that winter in the Yangtze River Basin and migrate over the East Asian-Australasian Flyway. We tested whether a decrease in functional connectivity was correlated with a decrease in non-breeding population sizes of migratory waterfowl species from 20012014. Additionally, we included six common predictors for population dynamics: breeding range size, migratory dispersion, migration distance, body mass, generation length, and clutch size. The results can provide new insights into the underlying mechanisms that affect population trends of migratory species, and point out efficient conservation strategies for safeguarding the sustainability of migratory birds under observed land use changes.

\subsection{Methods}

\section{Estimate bird population sizes}

We selected eight waterfowl species that have a majority of their East AsianAustralasian flyway population wintering in the Yangtze River Basin, China 87: tundra swan (Cygnus columbianus), swan goose (Anser cygnoid), bean goose (Anser fabalis), greater white-fronted goose (Anser albifrons), lesser white-fronted goose (Anser erythropus), greylag goose (Anser anser), common teal (Anas crecca), and northern pintail (Anas acuta). The flyway population trends for each of these species were estimated by Anatidae non-breeding counts between 2001-2014 in the Yangtze River Basin ${ }^{145}$. The non-breeding population sizes of each year were estimated by the sum of birds counted in all lakes in the Yangtze River Basin, and trends in population sizes were illustrated using a Locally Weighted Scatterplot Smoothing (LOWESS) method. Lakes without counting data in certain years were interpolated by means of nearest neighbours. The eight study species in a total of 24 lakes were observed annually during the 14-year study period during 2001-2014; thus, we included a total of 2688 records in the analysis.

\section{Quantify functional connectivity of migration networks}

For every year (1992-2015), we constructed migration networks of each of the study species, in which the 'nodes' were the connected wetland patches in the suitable sites for each study species in the East Asian-Australasian 
Flyway (Appendix 3.1; Xu et al. 2019). Distances between any two nodes were set at $>32.5 \mathrm{~km}$, which is the mean of maximum foraging flight distance of geese and ducks ${ }^{103}$. We defined the strength of node-to-node connections by the dispersal probability of the study species' direct movement between two nodes (inter-node dispersal probabilities). Because a limited number of birds have been ringed or fitted with telemetry equipment, we could not rely on direct calculations of dispersal probabilities between sites. Therefore, we used an indirect method, a decreasing exponential function ${ }^{146}$, to quantify inter-node dispersal probabilities.

$$
P_{i j}=e^{-k d_{i j}}
$$

$P_{i j}$ is the dispersal probability between node $i$ and $j$, and $d_{i j}$ is the closest distance between habitat patch $i$ and $j . k$ is a constant defined so that dispersal probability is $50 \%$ when $d_{i j}$ equals the median of the migration lags of the study species, i.e., distances between consecutive sites used by migratory birds (Appendix S3.3).

To facilitate comparison of functional connectivity of migration networks between years and species, we measured the functional connectivity of a migration network through an index of "equivalent connected area" 57,70 in $\mathrm{km}^{2}$ using a directed graph theory algorithm ${ }^{57}$. Equivalent connected area is the size of a single patch providing the same level of connectivity as the calculated migration network (equation 3.2). A larger equivalent connected area equates to a better-connected migration network.

$$
E C A=\sqrt{\sum_{i=1}^{n} \sum_{j=1}^{n} a_{i} a_{j} P_{i j}^{*}}
$$

$a_{i}$ and $a_{j}$ represent the area $\left(\mathrm{km}^{2}\right)$ of habitat sites (i.e., nodes) $i$ and $j . P_{i j} *$ is the maximum product probability, i.e., the "best" paths with one or more steps between nodes $i$ and $j$. When $P_{i j}{ }^{*}=P_{i j}$, nodes $i$ and $j$ are close enough to for individuals to move directly between them. When $P_{i j}{ }^{*}>P_{i j}$, the "best" path consists of several steps within the network and involves stepping stones in between nodes $i$ and $j$. The functional connectivity values were decomposed (contribution \%) into intra, direct and step fractions, showing the contribution (\%) of within-patch connectivity, direct connections, and use of stepping stones between source and destination patches, respectively 57. Specifically, intra-patch connectivity is the fraction corresponding to the area of reachable habitat within sites used by migratory birds. Direct 
connectivity is the amount of inter-site connectivity if stepping stones are not used in the movements of birds among sites. Step connectivity is the increase in the amount of connectivity by having higher probabilities of movement between sites due to the use of existing stepping stones. We ran the analyses in R 3.3.1 with Conefor 1.1.6 for directed networks ${ }^{148}$.

\section{Additional predictors for bird population changes}

We included six species traits as potential predictors in the analyses, i.e., body mass $(\mathrm{g})$, breeding range size $\left(\mathrm{km}^{2}\right)$, clutch size (the average number of eggs laid; $N$ per female), generation length (year), migratory dispersion, and migration distance $(\mathrm{km})$. Migratory dispersion is the size of species nonbreeding range relative to that of breeding range ${ }^{56}$. We calculated migratory dispersion, breeding range size, and migration distance on the basis of species' distribution maps ${ }^{95}$. We measured both migratory dispersion and breeding range size using a cylindrical equal area projection. Breeding range size was the total area of the whole breeding range of a study species. Migratory dispersion was measured by the difference between the logtransformed area of the non-breeding area and that of the breeding ranges divided by log-transformed area of the breeding ranges of a study species ${ }^{56}$. We measured migration distance as the distance between the centroids of breeding and non-breeding ranges of a study species using an azimuthal equidistant projection. Body mass and clutch size of the study species were obtained from the amniote life history database ${ }^{149}$. Generation lengths were obtained from the IUCN Red List of Threatened Species ${ }^{150}$.

\section{Statistical analysis}

We tested for differences in functional connectivity of migration networks among the different study species using a one-way ANOVA test followed by Tukey's post-hoc tests. Residuals were normally distributed (KolmogorovSmirnov test, $\mathrm{P}>0.05)$.

Phylogenetic non-independence among species can bias results, and we therefore implemented a phylogenetically corrected model, i.e., a multivariable Phylogenetic Linear Mixed Model ${ }^{151}$, to test for the effects of functional connectivity of migration networks and other additional predictors on the population changes of the study species. Data of all study species in each of the survey years (14 years; 2001-2014) were included in the PLMM. We fitted random effect terms that account for phylogenetic covariance ${ }^{152}$, bird species, and year of observation. We acquired a subset tree of the study species based on Ericson backbone ${ }^{153}$ from BirdTree.org ${ }^{154}$. 
The dependent variable was the population change ratio (PCR; equation 3.3). $P C R$ was calculated as the difference between the population size in a given year $i\left(P_{i}\right)$ and the population size of a starting year $\left(P_{2001}\right)$ divided by the population size of the starting year.

$$
P C R=\frac{P_{i}-P_{2001}}{P_{2001}}
$$

Independent variables (described above) included body mass, breeding range size, migratory dispersion, clutch size, generation length, migration distance, and changes in functional connectivity of migration networks of each species. Changes in functional connectivity (CFC) were calculated as the difference in functional connectivity of migration networks between a given year $i\left(F C_{i}\right)$ and the starting year $\left(F C_{2001}\right)$, equation 4 .

$$
C F C=F C_{i}-F C_{2001}
$$

We removed one independent variable with a variance inflation factor larger than 10 (generation length) to reduce the effect of multi-collinearity. All factors were scaled to facilitate comparison of the contributions to the prediction. Interaction terms were not fitted in the model due to limited sample sizes. The best model with the smallest Bayesian Information Criterion (BIC) was selected by a backward elimination procedure. To account for lag effects of changes in functional connectivity of migration networks on changes in population sizes, we fitted the PLMMs with different lag periods (Table 3.2; Appendix S3.2). In these lag models (Table 3.2), the change in functional connectivity $C F C(\operatorname{Lag} n)$ was calculated as the difference in functional connectivity $(F C)$ between a number of years (i.e. the number of lags: $n$ ) before the bird survey year $(i)$ and year 1992, the starting year of the functional connectivity measurement (equation 3.5).

$$
C F C(\operatorname{Lag} n)=F C_{i-n}-F C_{1992}
$$




\subsection{Results}

\section{Differences among species}

Average functional connectivity of migration networks over the period 19922015 significantly differed among the eight study species (Figure 3.1a; oneway ANOVA, $\left.F_{7,184}=23667, P<0.001\right)$. Among these species, the migration network of the northern pintail (Anas acuta) was the least connected with an equivalent connected area of $601,000 \mathrm{~km}^{2}$. The greylag goose (Anser anser, equivalent connected area $=908,000 \mathrm{~km}^{2}$ ), swan goose (Anser cygnoid, equivalent connected area $=871,000 \mathrm{~km}^{2}$ ), and common teal (Anas crecca, equivalent connected area $=831,000 \mathrm{~km}^{2}$ ) had the largest connected migration networks (Figure 3.1a).

Intra-patch connectivity contributed more than half to the functional connectivity of migration networks for all species. Step connections contributed least, especially for the swan and goose species with major breeding grounds in Russia (i.e., $2 \%$ for greater white-fronted goose (Anser albifrons), 4\% for bean goose (Anser fabalis), $5 \%$ for lesser white-fronted goose (Anser erythropus), and 5\% for tundra swan (Cygnus columbianus; Fig. $2 b)$.
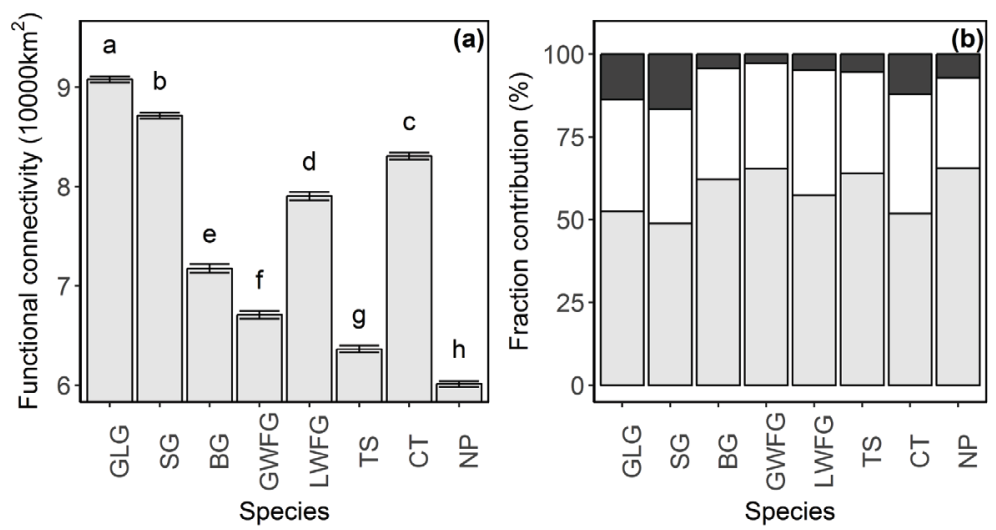

Figure 3.1 Functional connectivity of migration networks among bird species. (a) The eight study species include the greylag goose (GLG) and swan goose (SG), bean goose (BG), greater white-fronted goose (GWFG), lesser white-fronted goose (LWFG),

tundra swan (TS), common teal (CT), and northern pintail (NP). The equivalent connected area $\left(\mathrm{km}^{2} \times 10,000\right)$ averaged over $1992-2015$ is presented per species (bars) \pm the standard deviation (error bars). Letters represent group differences as identified by Tukey's multiple comparison test $(P \leq 0.05)$. (b) Contribution of fractions show intra-patch (light grey), direct (white), and step (dark grey) connections in percentages of functional connectivity. 


\section{Changes over time}

During 2001-2014, the estimated non-breeding population sizes of the greylag goose, swan goose, tundra swan, common teal, and northern pintail first increased and then decreased; population sizes of the bean goose, greater white-fronted goose, and lesser white-fronted goose first decreased and then either stabilized or slightly increased over the survey period (Figure 3.2). Generally, the study species showed a decreasing trend, and at the meantime, the functional connectivity of migration networks of all the eight species consistently and continuously declined since 2001. However, before 2001 (i.e., 1994-1999; periods differ among species), there was an increase in the functional connectivity.

\section{Loss of functional connectivity affects bird population dynamics}

Among the seven analysed factors, generation length was collinear with other predictors (variance inflation factor $>10$ ), and was excluded from the PLMM. The population change ratio was significantly and positively related to changes in functional connectivity of migration networks $(N=112$, regression coefficient $=0.24,95 \%$ confidence interval $=0.04-0.44, P=0.02$; Fig. 4a; Table 1). No other variables showed a significant effect, and changes in functional connectivity was the only factor included in the best model (Table 3.1). When the functional connectivity declined, bird populations declined (Figure 3.3b). There was a lag effect of functional connectivity on population changes of migratory birds, with a strongest effect of a 4-year lag on the population change ratio $(N=112$, regression coefficient $=0.26,95 \%$ confidence interval $=0.07-0.44, P=0.01$; Table 3.2; Appendix S3.2) . 
(a) Greylag goose

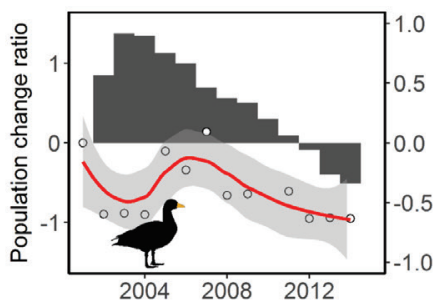

(c) Bean goose

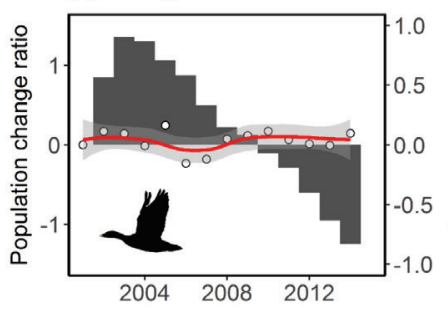

(e) Lesser white-fronted goose

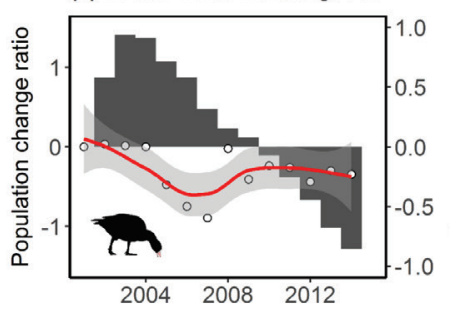

(g) Common teal

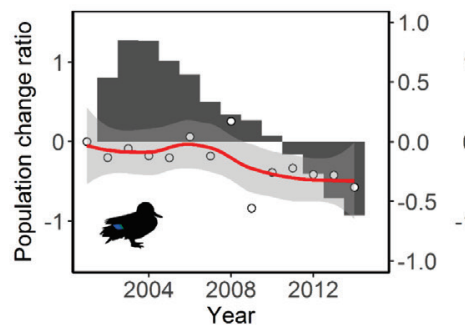

(b) Swan goose

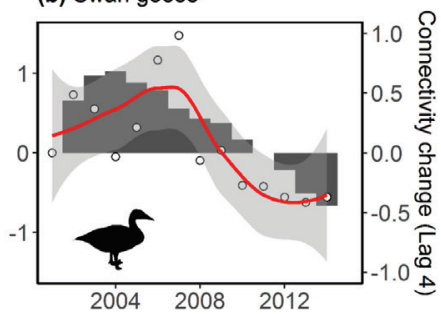

(d) Greater white-fronted goose

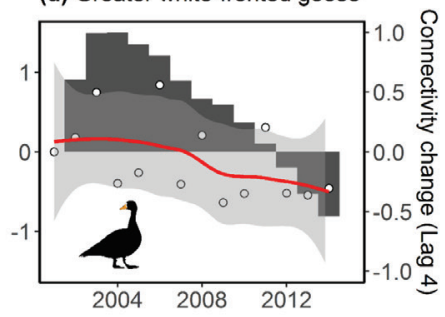

(f) Tundra swan

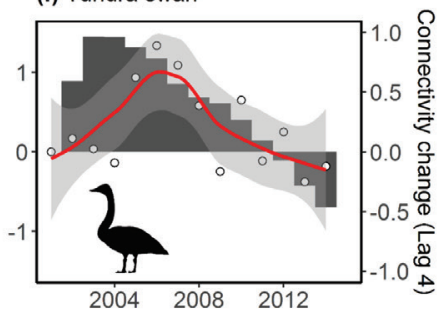

(h) Northern pintail

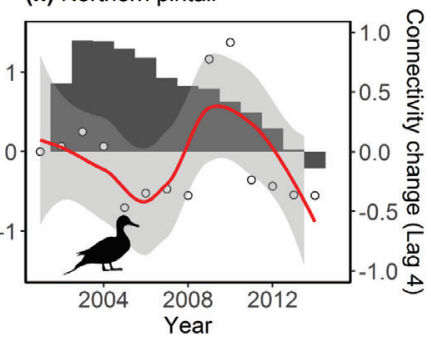

Figure 3.2 Changes in bird population sizes and in functional connectivity of migration networks. Population change ratio is the difference between the population size in a given year and the population size in 2001 divided by the population size in 2001. Population change ratios are displayed with dots and their trends are represented by smoothed red lines, using a Locally Weighted Scatterplot Smoothing method. Connectivity change (grey bars) with a 4-year lag is presented as the difference in the equivalent connected area $\left(\mathrm{km}^{2}\right)$ of the migration network between four years before a given year and year 1992. To facilitate comparison between species, connectivity change is standardized by being divided by the maximum connectivity change. A 4-year lag is displayed because the population changes are best explained by the changes in functional connectivity four years before the survey year (Table 2). 

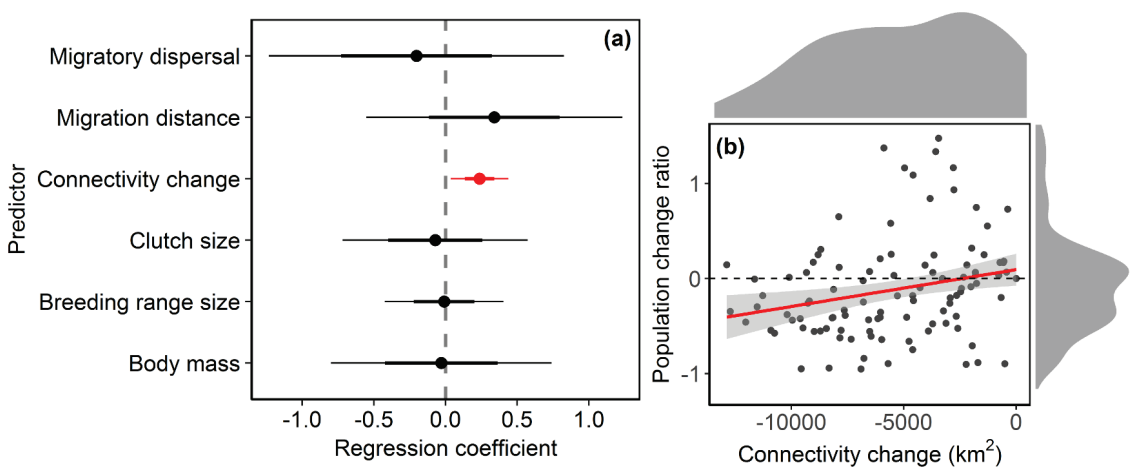

Figure 3.3 Changes in functional connectivity of migration networks is the only significant predictor for population declines of migratory birds. (a) Estimated coefficients $\pm 95 \%$ and $68 \%$ confidence interval (thick lines) of predictors for population change ratio using a full model of multi-variable phylogenetic Linear

Mixed Model. The significant predictor is in red. (b) The effect of changes in functional connectivity on population change ratios of the eight study species. We measured population change ratios using 2001 as the base line year, the first year of the bird survey. The relationship between population change ratio and changes

in functional connectivity were represented by a fitted line (red line) with $95 \%$ confidence intervals (grey area). Density plots on top and right show distributions of connectivity change and population change ratio, respectively.

Table 3.1 Results of the Phylogenetic Linear Mixed Model of species traits on changes in population sizes for 8 waterfowl species, showing the performance of the models and regression coefficients (Coefficient) for predictors included in these models. The full model and the best models with a smallest BIC are listed. "**” means the estimated regression coefficient was significant at $\mathrm{p}=0.05$.

\begin{tabular}{lclll}
\hline Model & Coefficient & $\begin{array}{l}\text { Standard } \\
\text { Error }\end{array}$ & z-value & p-value \\
& & & \\
\hline Full model (N = 112, BIC = 324.2) & 0.199 & 0.450 & 0.442 & 0.658 \\
$\quad$ Intercept) & -0.030 & 0.393 & -0.076 & 0.940 \\
$\quad$ Body mass & -0.010 & 0.211 & -0.048 & 0.962 \\
$\quad$ Breeding range size & 0.238 & 0.103 & 2.308 & 0.021 * \\
$\quad$ Changes in functional & & & & \\
connectivity & -0.072 & 0.329 & -0.219 & 0.827 \\
$\quad$ Clutch size & 0.341 & 0.456 & 0.748 & 0.455 \\
$\quad$ Migration distance & -0.203 & 0.525 & -0.386 & 0.699 \\
$\quad$ Migratory dispersal & 0.169 & 0.256 & 0.661 & 0.508 \\
Best model (N = 112, BIC $=320.7)$ & 0.100 & 2.163 & 0.031 * \\
$\quad$ Intercept) & 0.216 & & & \\
$\quad$ Changes in functional & & & & \\
connectivity & &
\end{tabular}


Table 3.2 A test for lag effects of changes in functional connectivity on changes in population sizes for 8 waterfowl species, showing the performance of the best models and regression coefficients (Coefficient) for predictors included in these models. The lag periods for measuring changes in functional connectivity are in a unit of years, i.e., in the model 'Lag 1', changes in functional connectivity were measured by the change one year before the corresponding population count. The number of lags represents the changes in functional connectivity over the number of years. "*" means the estimated regression coefficient was significant at $\mathrm{p}=0.05$.

\begin{tabular}{|c|c|c|c|c|}
\hline Model & Coefficient & $\begin{array}{l}\text { Standard } \\
\text { Error }\end{array}$ & $z$-value & $\begin{array}{l}\mathrm{p}- \\
\text { value }\end{array}$ \\
\hline \multicolumn{5}{|c|}{ Lag $1(\mathrm{~N}=112, \mathrm{BIC}=319.9)$} \\
\hline (Intercept) & 0.175 & 0.253 & 0.691 & 0.490 \\
\hline $\begin{array}{l}\text { Changes in functional } \\
\text { connectivity }\end{array}$ & 0.235 & 0.100 & 2.359 & $\begin{array}{l}0.018 \\
*\end{array}$ \\
\hline \multicolumn{5}{|c|}{ Lag $2(N=112, B I C=319.4)$} \\
\hline (Intercept) & 0.175 & 0.253 & 0.693 & 0.488 \\
\hline $\begin{array}{l}\text { Changes in functional } \\
\text { connectivity }\end{array}$ & 0.245 & 0.098 & 2.494 & $\begin{array}{l}0.013 \\
*\end{array}$ \\
\hline \multicolumn{5}{|c|}{ Lag $3(N=112, \mathrm{BIC}=318.9)$} \\
\hline (Intercept) & 0.175 & 0.250 & 0.699 & 0.484 \\
\hline $\begin{array}{l}\text { Changes in functional } \\
\text { connectivity }\end{array}$ & 0.254 & 0.095 & 2.674 & $\begin{array}{l}0.007 \\
*\end{array}$ \\
\hline \multicolumn{5}{|c|}{ Lag $4(N=112, \mathrm{BIC}=318.8)$} \\
\hline (Intercept) & 0.175 & 0.249 & 0.703 & 0.482 \\
\hline $\begin{array}{l}\text { Changes in functional } \\
\text { connectivity }\end{array}$ & 0.257 & 0.094 & 2.749 & $\begin{array}{l}0.006 \\
*\end{array}$ \\
\hline \multicolumn{5}{|c|}{ Lag $5(N=112, \mathrm{BIC}=319.1)$} \\
\hline (Intercept) & 0.176 & 0.249 & 0.705 & 0.481 \\
\hline $\begin{array}{l}\text { Changes in functional } \\
\text { connectivity }\end{array}$ & 0.252 & 0.094 & 2.668 & $\begin{array}{l}0.008 \\
*\end{array}$ \\
\hline \multicolumn{5}{|c|}{ Lag $6(N=112, \mathrm{BIC}=319.2)$} \\
\hline (Intercept) & 0.177 & 0.250 & 0.708 & 0.479 \\
\hline $\begin{array}{l}\text { Changes in functional } \\
\text { connectivity }\end{array}$ & 0.249 & 0.093 & 2.677 & $\begin{array}{l}0.007 \\
*\end{array}$ \\
\hline \multicolumn{5}{|c|}{ Lag $7(N=112, B I C=321.9)$} \\
\hline (Intercept) & 0.173 & 0.252 & 0.688 & 0.492 \\
\hline $\begin{array}{l}\text { Changes in functional } \\
\text { connectivity }\end{array}$ & 0.185 & 0.101 & 1.824 & 0.07 \\
\hline
\end{tabular}




\subsection{Discussion}

We found that loss of functional connectivity in migration networks is a crucial predictor for population declines in migratory birds. Changes in functional connectivity was the only significant factor in the model predicting population changes, outperforming other previously used predictors of population decline and local extinction of migratory birds. Although migratory birds have high flexibility in distribution and migration, their populations declined with a decrease in functional connectivity. Moreover, population sizes responded immediately to connectivity loss, however, when a lag effect was taken into account in the analysis, the impact of changes in functional connectivity on population sizes became even stronger (Table 3.2; Appendix S3.2). The impact of decreasing functional connectivity on population sizes can last long, and was largest using a 4-year lag effect.

With the loss of connectivity in their migration network, migratory birds must either adapt to suboptimal resources or accept suboptimal strategies 89,93,155, e.g., longer non-stop flights and/or suboptimal arrival, departure and residence times. These adjustments could lead to increasing costs of migration and decreasing efficiency in energy refuelling 156; consequently, mortality during migration could increase, and breeding success could decline. Carryover effects 157 could result in conditions becoming tougher over time for those species that successively lose habitat area along their migration routes. Ultimately, this loss of functional connectivity in migration networks could make it difficult to replenish energy stores during migration and maintain optimal body reserves for reproduction 158 .

A well-connected network facilitates animal movements and subsequent survival and viability ${ }^{159}$, especially for migratory birds, which pass through long and narrow geographic ranges twice a year. As for the study species, greylag goose and swan goose with relatively wider and shorter migration extents, have relatively well-connected migration networks than the other species (e.g., northern pintail, tundra swan, and greater white-fronted goose). A well-connected migration network provides sufficient alternative routes for migrants, and not only promotes migratory movements, but also provides more possibilities for range shifts to cope with area-specific environmental changes. Migratory species that rely on a migration network that is continuously losing connectivity are more likely to experience a population decline. Hence, connectivity of migration networks is an essential 
element for habitat change analysis for migratory species. Establishment of systematic conservation networks across species migration extents based on international agreements, e.g., the Ramsar Convention on Wetlands of International Importance and the European Union's Biodiversity Strategy 2020, should be comprehensively considered for biodiversity conservation planning.

Stepping stones are essential during seasonal migration. Even during Sahara crossing, migratory birds take short diurnal stopovers in resourcepoor desserts 160 as stepping stones, and birds crossing the Himalayas do the same ${ }^{161}$. However, the step connections in the studied migration networks contributed least to the functional connectivity, which indicated a warning signal that these studied waterfowl species lack stepping stones to facilitate movements between sites. Successive loss of crucial stepping stones in migration routes can lead to the collapse of migration networks, so that migratory movements between breeding and non-breeding grounds could be completely impeded ${ }^{37}$. Thus, it is necessary to put an emphasis on protecting critical sites used as stepping stones in migration to enhance the connectivity between isolated sites.

Human-induced and climate-driven changes to natural land covers can have large impacts on the connectivity of animal movement networks. Composition and structure of landscape mosaics can explain large-scale species distribution and richness patterns especially for birds 81,162 . As indicated by our study, the functional connectivity of migration networks for species in the East Asian-Australasian Flyway was continuously decreasing from 2000s. Wetlands are one of the world's mostly threatened habitat types, under influence of climate change and human-induced habitat destruction 75,76 . Wetland loss can isolate and eliminate habitat sites in migration networks, thereby reducing network connectivity. Under high human-bird conflicts, wetlands in this region are widely destroyed by human activities.

Migratory birds that rely the most on degraded stopover sites experienced the largest population decline ${ }^{35}$, and habitat conditions during migration can influence bird survival ${ }^{163}$. Decreasing wetland area and food availability (e.g., via loss of grasslands) can lead to staging sites no longer being utilized by migrants 164,165 . The vulnerability of a migratory species that uses a number of wetland sites increases even when only part of the network is negatively affected (e.g., by sea-level rise or other human-induced 
changes ${ }^{73}$. Upon the degradation or loss in individual sites, the chance of a breakdown in both direct and indirect connections between sites increases. It is therefore essential to maintain well-connected habitat networks by either expanding the area of (protected) sites or by adding new sites to existing networks, to increase the resilience of migrants to environmental change.

Species traits are often associated with population dynamics, as species that vary in traits respond differently to environmental changes. Individuals of large-bodied species might be able to adapt to environmental changes more easily, as they are physiologically more resistant to environmental changes. Thus, species with larger body mass may have an advantage when environmental conditions deteriorate. The connectivity of the studied migration networks decreased continuously over the past 15 years, under influence of deteriorating environmental conditions, such as habitat loss and fragmentation. However, the expectation that larger species were less affected by the decrease in function connectivity of their migration network could not be confirmed in our study, as the decline in population was similar among species. This in in agreement with, for example, the findings of 144 who also found no relationships between the species body mass and their population trends. The extent to which the species' life-history traits affect their population dynamics is dependent on the geographical ranges and orders of species studied. We fitted species as a random factor in the PLMM, the signal of the species traits may therefore be weakened. Our results indicate that a loss of functional connectivity of their migration network is a novel and crucial predictor for population declines of migratory birds. Further studies about population declines of migratory birds should take this factor into account. However, other species traits may also be important factors triggering population dynamics of migratory birds. This study included waterfowl species in the East Asian-Australasian flyway, so future researches investigating whether our findings can be applied to a broader geographical or species range will be valuable. 


\subsection{Acknowledgements}

We thank Kevin Matson (Wageningen University, the Netherlands) for his insightful comments on the manuscript, and Henjo de Knegt (Wageningen University, the Netherlands) for help with the spatial analysis. Financial support was provided by the National Key R\&D Program of China (No. 2017YFA0604404), the National Natural Science Foundation of China (No. 41471347) and Chinese Scholarship Council (No.201600090128). We declare no competing interests. 


\section{Supplementary information}

Appendix S3.1 Patterns of habitat loss in the ranges of study species. The suitable sites in the East Asian-Australasian Flyway for each study species and change ratios of the area of wetlands in the suitable sites during 1992-2012 were both analysed in a previous study ${ }^{147}$. Each connected wetland patch (within a distance of $32.5 \mathrm{~km}$ ) in the suitable sites was defined as a node of the migration networks. The map was produced with ArcMap 10.2 under the cylindrical equal area projection.
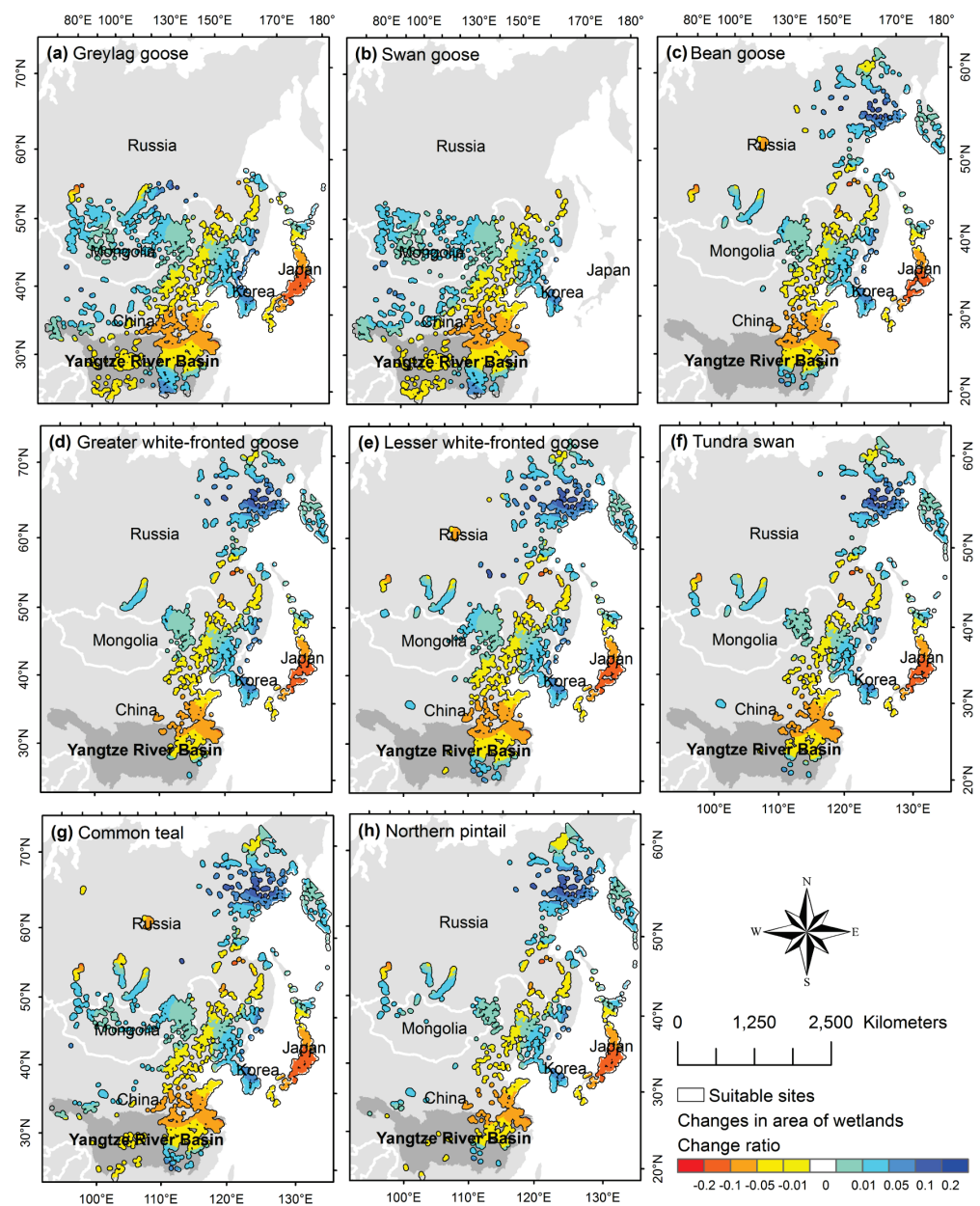
Appendix S3.2 Effect of lag period on the relationship between functional connectivity of migration networks and changes in population sizes of migratory birds. Functional connectivity was calculated for different lag periods (years, $\mathrm{x}-$ axis). Estimated coefficients ( \pm standard error) of the correlation between changes in functional connectivity and population sizes were obtained from multi-variable Phylogenetic Linear Mixed Models. The default model is with 0 lag and the dashed

line represent the estimated coefficient from the default model to facilitate comparison between models. N.S. = not significant.

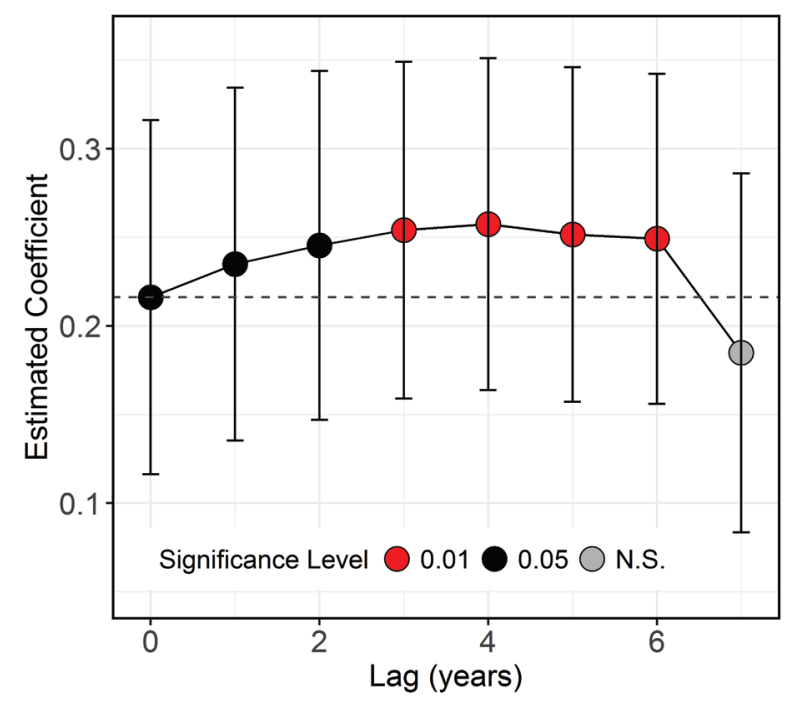

Appendix S3.3 Migration step length, i.e., distances between consecutive sites from tracking studies. 'Species group' stands for the category of species (geese, swans, or ducks), and major breeding grounds, i.e., border area between Mongolia, China, and Russia (geese 1) or Russia (geese 2). Common name and scientific name of focal species. Migration step length is the median distance (in $\mathrm{km}$ ) of the migration steps between consecutive sites for a study species, as published in previous studies. For species where we could not find information on the median distance between steps in their migration network we took the value as published for the "indicator species".

\begin{tabular}{|c|c|c|c|c|}
\hline $\begin{array}{l}\text { Species } \\
\text { group }\end{array}$ & $\begin{array}{l}\text { Common name of } \\
\text { study species }\end{array}$ & $\begin{array}{l}\text { Scientific } \\
\text { name }\end{array}$ & $\begin{array}{l}\text { Indicator } \\
\text { species }\end{array}$ & Migration step length $(\mathrm{km})$ \\
\hline \multirow[t]{2}{*}{ Geese 1} & Greylag Goose & Anser anser & Anser cygnoides & 13090 \\
\hline & Swan Goose & $\begin{array}{l}\text { Anser } \\
\text { cygnoides }\end{array}$ & Anser cygnoides & 13090 \\
\hline \multirow[t]{3}{*}{ Geese 2} & Bean Goose & Anser fabalis & Anser albifrons & 550166 \\
\hline & $\begin{array}{l}\text { Greater White- } \\
\text { fronted Goose }\end{array}$ & Anser albifrons & Anser albifrons & 550166 \\
\hline & $\begin{array}{l}\text { Lesser White- } \\
\text { fronted Goose }\end{array}$ & $\begin{array}{l}\text { Anser } \\
\text { erythropus }\end{array}$ & Anser albifrons & 550166 \\
\hline Swans & Tundra Swan & $\begin{array}{l}\text { Cygnus } \\
\text { columbianus }\end{array}$ & $\begin{array}{l}\text { Cygnus } \\
\text { columbianus }\end{array}$ & 571167 \\
\hline \multirow[t]{2}{*}{ Ducks } & Common Teal & Anas crecca & Anas acuta & 258168 \\
\hline & Northern Pintail & Anas acuta & Anas acuta & $258^{168}$ \\
\hline
\end{tabular}


Loss of functional connectivity in migration networks induces population decline in migratory birds | 63 



\section{Indicators of site loss from a migration network: anthropogenic factors influence waterfowl movement patterns at stopover sites}

Xu Y., Kieboom M., van Lammeren R. J. A., Si Y., de Boer W. F. Indicators of site loss from a migration network: anthropogenic factors influence waterfowl movement patterns at stopover sites. Global Ecology and Conservation [Under Review]. 


\section{Abstract}

A migration network of connected habitats supports migratory movements of waterfowl. These networks are rapidly changing due to intensive human activities around natural habitats. Quantifying how anthropogenic factors change waterfowl movements via a reduction of habitat availability and quality can facilitate a better understanding of the dynamics of these migration networks, and early-warning signals for network collapse. Using satellite tracking data for greater white-fronted geese (Anser albifrons) in the East Asian-Australasian Flyway, we tested how anthropogenic and ecological factors influence geese movement patterns at stopover sites. We found that these factors, e.g., percentage of farmlands in the landscape, proximity index of wetland patches, accurately predicted percentage of flying time, and the median movement distance of tracked geese at stopover sites. Farmlands negatively affected the habitat quality because the geese flew more frequently, made longer movements, and switched their behaviour more frequently in landscapes with a higher proportion of farmlands. Goose movements were constrained in natural habitats, as a higher proportion of water and wetlands increased their movements, and thereby increased flying time and median movement distances. This analytic approach provides a quantitative tool to measure quality of stopover sites, which can be used as an index for the probability of losing a site from a migration network, and thereby generates insights for setting priorities in conservation planning of migratory waterfowl. 


\subsection{Introduction}

Human practices may have both positive and negative effects on waterfowl survival and foraging success in local habitats 51,119 , and ultimately modify the structure of their migration network ${ }^{169}$, i.e., a habitat network of nonbreeding, breeding, and a number of stopover sites which supports bird migration ${ }^{170}$. Under global environmental changes, suitability of these sites may decrease over time, and consequently a site may become no longer used by migratory birds. Habitat loss and degradation reduce the connectivity of migration networks, negatively affecting migration success, and subsequent reproduction of migratory birds 35,169 . The human influence on waterfowl is increasing in recent decades, and human practices in areas around wetland sites, and urban sprawl are the major agents reducing local habitat availability and suitability for waterfowl, and thereby modify network configuration.

However, in some cases waterfowl species take advantage of human activities when they provide them with profitable food resources. For example, waterfowl utilizes agricultural regions in Europe and North America, where short, rapidly growing and high quality swards are created for cattle grazing by high input of fertilizers and sowing of specific early growing and high productive grass species ${ }^{83,119}$. In East Asia, geese also utilize croplands in their stopover sites as alternative feeding patches, although other foraging lands (e.g., grassland and wetland) are preferred 81,89. Moreover, some bird species may adapt to anthropogenic influence, for instance, brent geese (Brenta bernicla) forage on heavily disturbed wintering habitats with high food availability ${ }^{171}$. Some shorebirds select flooded rice paddies over natural wetlands during their early stages of overwintering in Colusa National Wildlife Refuge ${ }^{172}$. Local movements of birds may not be restricted by the urban landscapes surrounding habitat patches, as the proximity between habitat patches is the dominant factor explaining movements for e.g., weaver birds ${ }^{32}$.

Nevertheless, waterfowl species are often negatively affected by human activities in and around their habitats. Human-related disturbance may result in farther within-habitat movements, shorter foraging and roosting durations, and frequent switches between habitat patches, and these responses could negatively affect their energy storage during migration, and subsequently impact their survival and breeding outputs 173,174 . Human disturbance triggers escape flights, and forces birds to spend more time on 
vigilance instead of foraging and roosting, which subsequently decreases the efficiency of their resting and refuelling, and increases their energy expenditure ${ }^{175}$. Some sensitive species might even shift their habitat from a highly disturbed area to a less disturbed but also less energy-profitable area, decreasing their foraging efficiency, or leading to suboptimal diets ${ }^{171}$. In addition, wetland reclamation may result in habitat degradation and fragmentation, which impact migratory waterfowl by forcing them to move more frequently between smaller and more isolated habitat patches.

Understanding how waterfowl movement patterns change in response to these human activities generates insights into the effect these activities have on waterfowl habitat quality. Moreover, this can advance our understanding of how these factors influence the structure of the migration network by adding new stopover sites, or removing previously suitable sites. Satellite tracking facilitates comparison of waterfowl movement patterns under different levels of human-related disturbance. In this study, the effects of different human activities on waterfowl's use and movements of and around stopover sites all along their migration route were investigated to provide an integrated view of waterfowl responses to environmental (i.e. a combination of ecological and anthropogenic) factors.

To better understand the direction and extent to which environmental factors affect waterfowl movements at their stopover sites during migration, satellite tracking data of greater white-fronted geese (Anser albifrons) was obtained. The tracking data was separately analysed at each stopover site, and geese movement patterns were quantified as indicators of waterfowl responses to human activities and ecological factors. These geese are hypothesized to be negatively influenced by most anthropogenic practices including land reclamation, urbanization, and transportation, although they could also benefit from agricultural practices by grazing in croplands. A high quality stopover sites is then expected to be correlated with a low movement distance, a low flying time, a high staging time, and a low frequency of switching between low and high movement speeds, which is associated with human disturbance (Figure 4.1). The results can assist in setting priorities for conservation actions, in quantifying the probability of losing a site from a migration network, and in generating early warning signal for network collapse. 


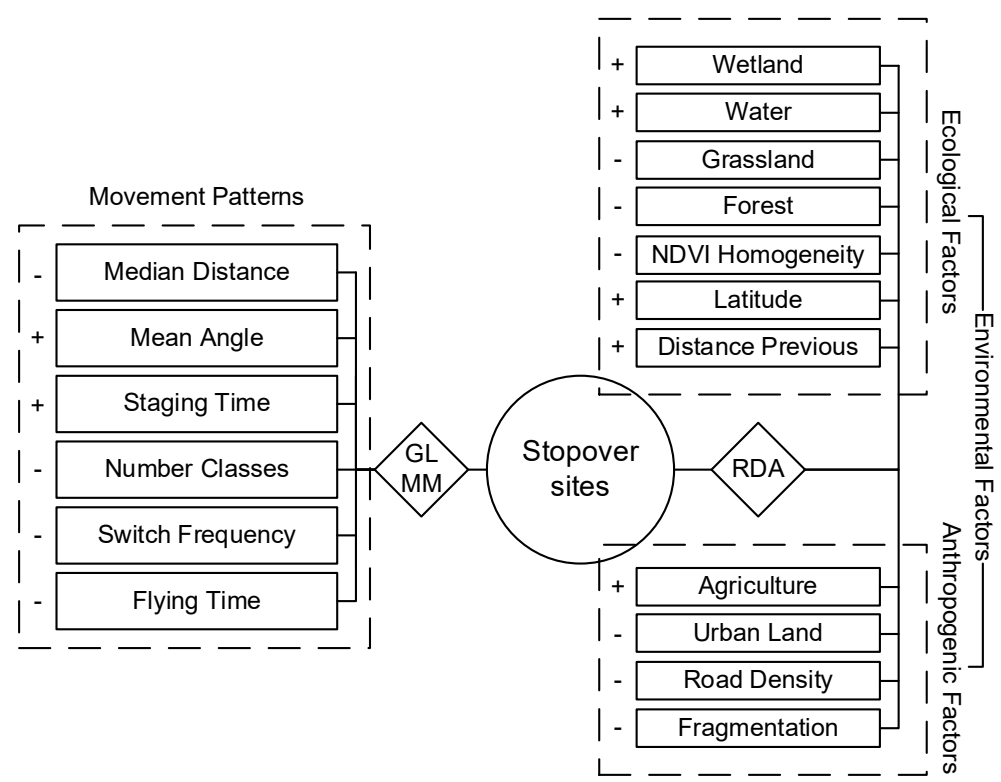

Figure 4.1 A framework for the effects of human activities on waterfowl movements. GLMM = General Linear Mixed Model; RDA $=$ Redundancy Analysis. A higher value of the movement patterns marked with "+" indicates a higher habitat quality with lower disturbance, while a higher value of those marked with "-" indicates a lower quality. The environmental factors marked with "+" were hypothesized to positively affect geese movement patterns related to high-quality habitats, while the ones marked with "-" were hypothesized to positively affect geese movement patterns related to low-quality habitats.

\subsection{Methods}

\section{Tracking data and land cover map}

The satellite tracking data for 21 greater white-fronted geese from 20152019 were used for movement pattern analysis in their stopover sites along their migration routes. The geese were captured in Poyang Lake, Jiangxi, China $\left(29^{\circ} \mathrm{N}, 116^{\circ} \mathrm{E}\right)$. The interval of GPS/GSM loggers was set as 1 position per $2 \mathrm{~h}$. The obtained mean daily count of GPS locations recorded was 10 positions per goose. We used latitude, longitude, date, and time for each GPS location for subsequent analyses. Details about geese capture, tracking, and ethics were shown in previous publications analysing this dataset 89,170 .

We obtained 300-m resolution land cover maps for 2015 from ESA CCI Land Cover time-series v2.0.7 (1992 - 2015) dataset. Nine land cover types were included in subsequent analyses: wetlands (land cover code: 180), water (210), agriculture $(10,11,12,20,30,40)$, urban (190), forest $(50,60$, 
$61,62,70,80,90,100,160,170)$, and grasslands $(110,130)$. We also obtained 300-m resolution normalized density vegetation index (NDVI) maps from Copernicus Global Land Service for the years 2015-2019. The temporal resolution of these maps is 10 days/image, so we were able to select the NDVI maps that matched the period of geese staging in each of the stopover site. For road density estimation, we obtained road maps from the Global Roads Open Access Data Set v1 (1980-2010).

\section{Identifying stopover sites}

The study area included stopover sites utilized by tracked geese during their northward spring and southward autumn migration. To identify these sites, we used the Guéguen method 176 to distinguish migration movements from non-migration movements. The Guéguen method used a Bayesian division for movement tracks, assigning a track (moving trajectory within a defined time period) to several segments, based on the changes in bird movement patterns, i.e., migration or non-migration ${ }^{177}$. The segments assigned to nonmigratory movements, were defined as breeding/non-breeding/stopover sites. We only included defined sites that were used for $\geq 48 \mathrm{~h}$ for subsequent analyses, under the assumption that a stopover site is used for at least 2 days ${ }^{89}$. Because we only focused on bird movements in stopover sites, we removed the southernmost and northernmost segments to exclude breeding and non-breeding sites.

\section{Analysing movement pattern}

We used the Guéguen method, again, to assign local movement tracks to different segments. These segments were classified into different movement patterns, ranging from 1 to 10 , from little to no movement (class 1 and class 2 ), to intermediate and excessive movement (class 3 or higher). With these outputs, we quantified several indicators of bird movement patterns at each stopover site: median distance, percentage of flying time, mean angle, frequency of switches between behavioural classes, number of behavioural classes, and total staging time.

Duration and timing. Animals maximize their net energy intake rate and hence spend more time in relatively richer patches ${ }^{178}$. Therefore, the duration of staging in a stopover site, termed staging time here, was calculated as one index to reflect the habitat quality. In highly disturbed situations, geese may spend more time on vigilant behaviour, or fly away, and seek shelter in less disturbed habitats, instead of foraging and roosting 175. Therefore, we quantified percentage of flying time (tracking points 
defined as 'flying' * sampling interval), as another index, by log (total amount of time a geese is flying/the total staging time per stopover site). We defined segments with intermediate and excessive movements (i.e. class 3 or higher) as 'flying'.

Distance and angle. In response to reduced habitat suitability, movements of waterbirds increase ${ }^{179}$, which is reflected in an increased flying distance, and thus the median distance (step length in meter) of each tracked bird in each stopover site was calculated. In addition, we calculated the median absolute turning angle for each tracked bird in each stopover site, as deviations from linear movements (i.e. larger turning angles) are more associated with foraging movements instead of large distance displacements ${ }^{180}$.

Behavioural switches. We assume a bird switches its behaviours frequently and displays more types of behaviours in low-quality stopover sites. We therefore quantified the frequency of behavioural switches, i.e. the frequency of switches between different behavioural classes (logtransformed). Additionally, we calculated the number of different behavioural classes a bird displayed within each stopover site.

\section{Quantify environmental factors at different spatial scales}

Quantification of the relationships between species and environment can be largely affected by spatial scales 81,181 . For a better understanding of the effect of environmental factors on bird movements, we therefore quantified the environmental factors that may affect the quality of a stopover site at different scales, i.e., 90\%, 95\%, and 99\% areas defined as follows. We used dynamic Brownian Bridge Movement Models (dBBMM) ${ }^{182}$, which measure bird home ranges based on a probability of their space use, to define the utilized ranges for the tracked geese at each stopover site. We define geographical ranges of $90 \%, 95 \%$, and $99 \%$ isopleths of the utilization distributions as mostly used, highly used, and total range of the utilized area for each site. For each of these scales in each site, we quantified the following environmental factors.

Anthropogenic factors. The main human-related effect agents for waterfowl include agricultural practices, land reclamation, urbanization, and transportation 183,184 . We calculated the percentage of agriculture lands and urban lands at each scale in each stopover site. We also measured the road density $\left(\mathrm{km} / \mathrm{km}^{2}\right)$, by the length of roads per square $\mathrm{km}$, as another 
factor indicating the intensity of human activities. We quantified habitat fragmentation by the proximity index of wetlands. A lower value indicates that habitat patches within a single stopover site are relatively isolated from each other ${ }^{112}$.

Ecological factors. Wetland availability and vegetation heterogeneity, also influence waterfowl use of stopover sites, and were therefore also included in our models. We calculated the percentage of wetlands, water, grasslands, and forests at each scale in each stopover site (\%). Water and wetlands are the most important natural habitats for geese ${ }^{101}$, so we additionally calculated the percentage of wetlands plus water. To measure the availability of roosting habitat and food resources, we calculated a logtransformed area of water and a log-transformed area of wetlands plus grasslands $\left(\left(\log \mathrm{km}^{2}\right)\right.$. We also measured surface area of stopover sites by the total area of each site in $\mathrm{km}^{2}$. We included NDVI heterogeneity in each of the sites as an additional factor which represent heterogeneity of food resources for waterfowl in a landscape. NDVI heterogeneity was calculated by the standard deviation of NDVI values in landscape in and around each stopover site, excluding urban lands and water which can highly skew values. Moreover, because birds get closer to their destinations, i.e., breeding/non-breeding grounds, their movements patterns may change, for example, stage less or more at stopover sites ${ }^{166}$. We also calculated the mean latitude of all tracking points per stopover site. Lastly, we measured the distance to the previous stopover (or breeding/non-breeding) site by the distance from the first GPS location in the focal site to the last location in the previous site.

To select the best scale for an environmental factor to explain a movement pattern variable, we fitted General Linear Models (GLM) for each pair of the movement pattern variable (independent variable) and the environmental factor (dependent variable) at the 90\%/95\%/99\% areas. Environmental factors at the areas with a lowest Akaike information criterion (AIC) in the model, i.e., the best-fitting scale, was applied in subsequent analyses.

\section{Statistical analyses}

To test the birds' responses to different ecological and anthropogenic factors, we fitted General Linear Mixed Models (GLMM) for each movement pattern variable (dependent variable) and all environmental factors (independent variables). The ID of tracked birds was used as a random factor in the 
GLMM. In case of a collinearity between the factors, we maintained only environmental factors with variance inflation factors (VIF) $\leq 5$ in the models. We selected the models with a lowest AIC for predicting movement patterns. To test how accurate these environmental factors can predict a site quality, we randomly selected $70 \%$ sites $(n=90)$ to train the models and used the remaining $30 \%$ sites $(n=39)$ to validate the models. To test the sensitivity of model outputs to these random selections of training (70\%) and testing (30\%) samples, each random selection was repeated for 5 times.

To summarize how environmental factors predict the site quality, we created a multivariate dataset consisting of movement pattern variables at different stopover sites and all environmental factors. Both positive and negative effects of different environmental factors can be incorporated in a Redundancy Analysis (RDA), which analyses relationships between more than one response variable (i.e. movement pattern variable) and explanatory (i.e. environmental) variables. The derived canonical axes extract most of the variation in movement patterns, together with the environmental factors that are best correlated with the changes in geese movements. All movement pattern variables and environmental factors were standardized to range from 0 to 1 before running the RDA. The analysis were conducted in R3.3.3.

\subsection{Results}

\section{Movement pattern responses to environmental factors}

The median distance, number of behavioural classes, frequency of behavioural switches, and flying time increased with an increasing percentage of agriculture lands, which was in line with our expectations. Also flying time increased with an increasing road density, and mean angle increased with an increasing percentage of urban lands. However, in some rare cases, human activities affected movement patterns in a different way, as the percentage of agricultural lands was negatively correlated with the mean angle, and the percentage of urban lands was negatively correlated with the median movement distance. The proximity of wetland patches within a stopover site (i.e. an index for fragmentation) was another important factor for predicting movement patterns of birds. However, in contrast to our predictions, proximity index was positively correlated with the number of behavioural classes (Table 4.1), frequency of behaviour switches, and flying time, while it had a negative effect on mean angle and staging time. 
Among ecological factors, surface area was the most important factor for explaining movement patterns, which was positively correlated with staging time, flying time, median distance, and number of behavioural classes, and was negatively correlated with mean angle. The percentage of water was positively correlated with median distance and flying time, while it was negatively correlated with the mean angle. The percentage of wetlands/grasslands was positively correlated with all movement pattern variables. NDVI heterogeneity was positively correlated with staging time and frequency of behavioural switches, while it negatively affected flying time.

\section{Validations for predictions of site quality}

Because there was no significant difference between the five different random selections, we explain here the results from the first random selection for model accuracy. The model for flying time made the most accurate prediction for the test dataset $\left(R^{2}=0.42\right.$; Figure $\left.4.2 b\right)$. The model for the median distance generated the best prediction for the training dataset $\left(R^{2}=0.74\right.$; Appendix $S 4.1$ a) and the overall dataset $\left(R^{2}=0.63\right.$; Appendix S4.1c). Compared to other movement variables, the environmental factors explained the least amount of variation for the mean turning angle $\left(R^{2}=0.06\right.$ for the overall dataset; Appendix S4.2).
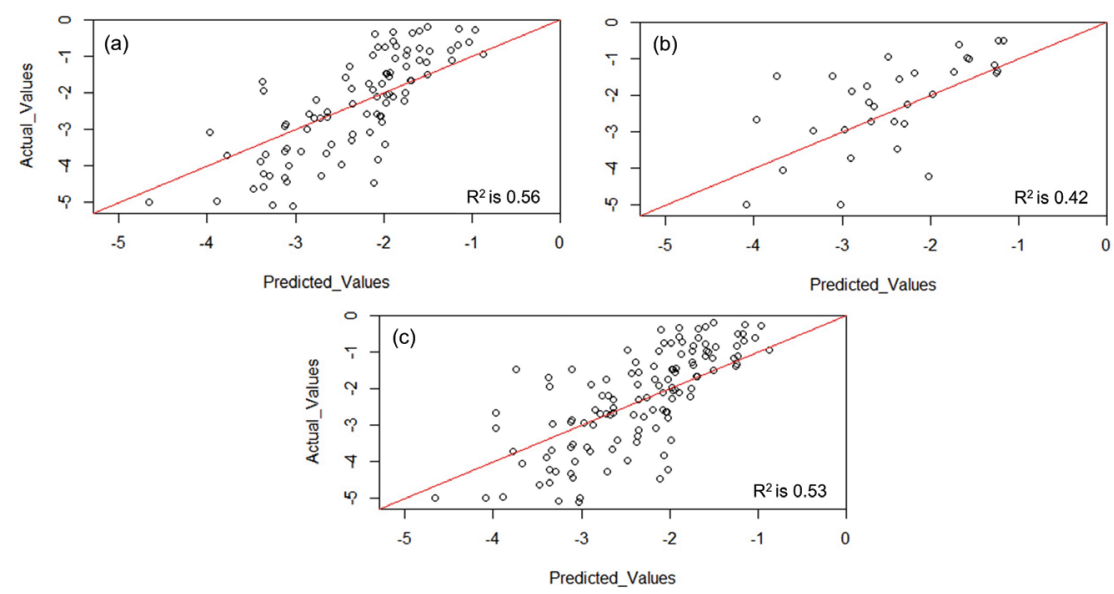

Figure 4.2 The predicted values for the log-transformed flying time in a site by the best model, plotted against the observed values of this variable. (a) the model with original dataset $(\mathrm{N}=90)$ which are the sites used to create the model, (b) the model with validation dataset $(\mathrm{b} ; \mathrm{N}=39)$ which are the sites that were randomly selected for validation, (c) the model with all data $(\mathrm{N}=129)$. The red lines indicate the $\mathrm{X}=\mathrm{Y}$ line (i.e. a perfect prediction), with the R-squared value reflecting the accuracy of the model compared to this line. 


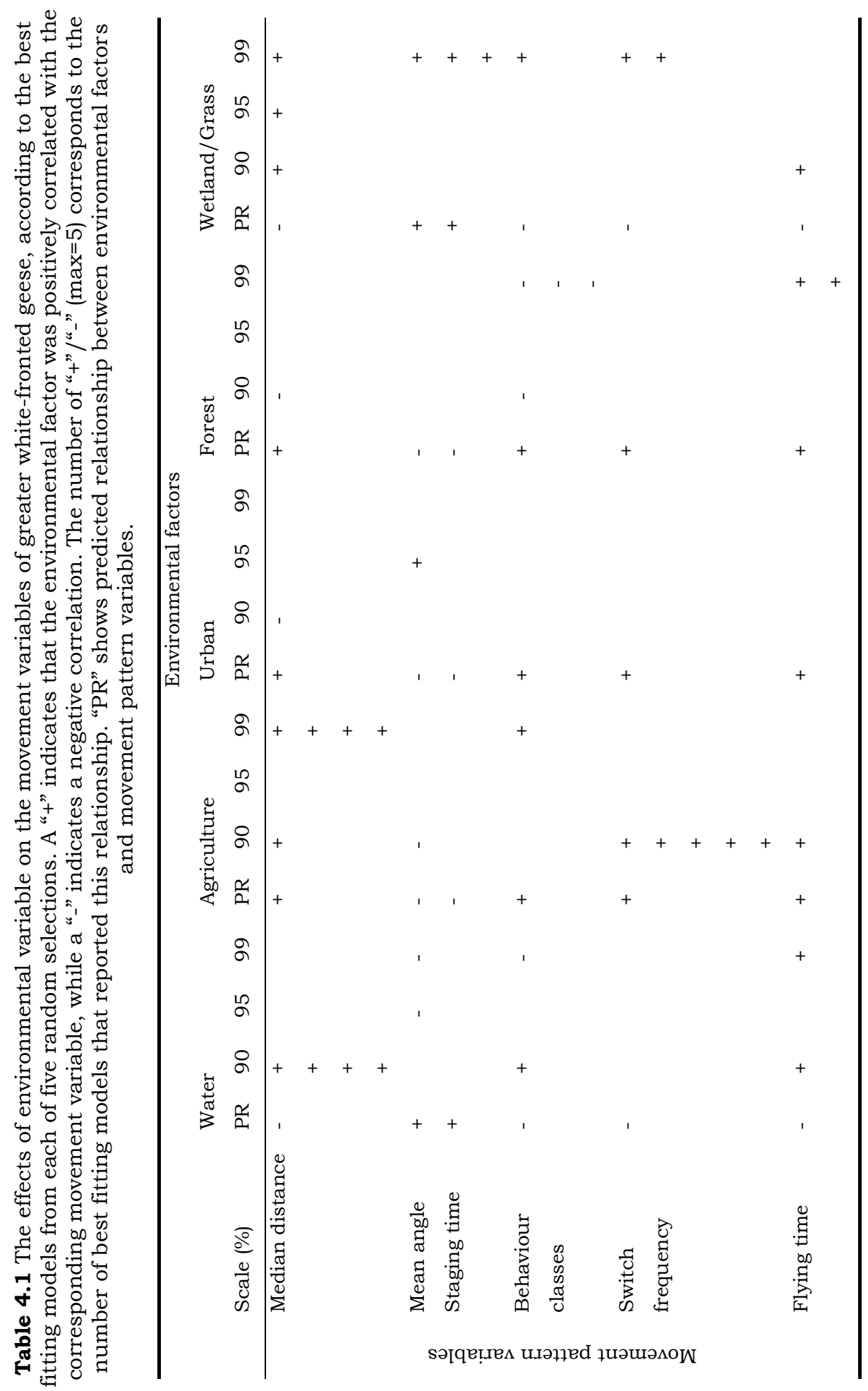




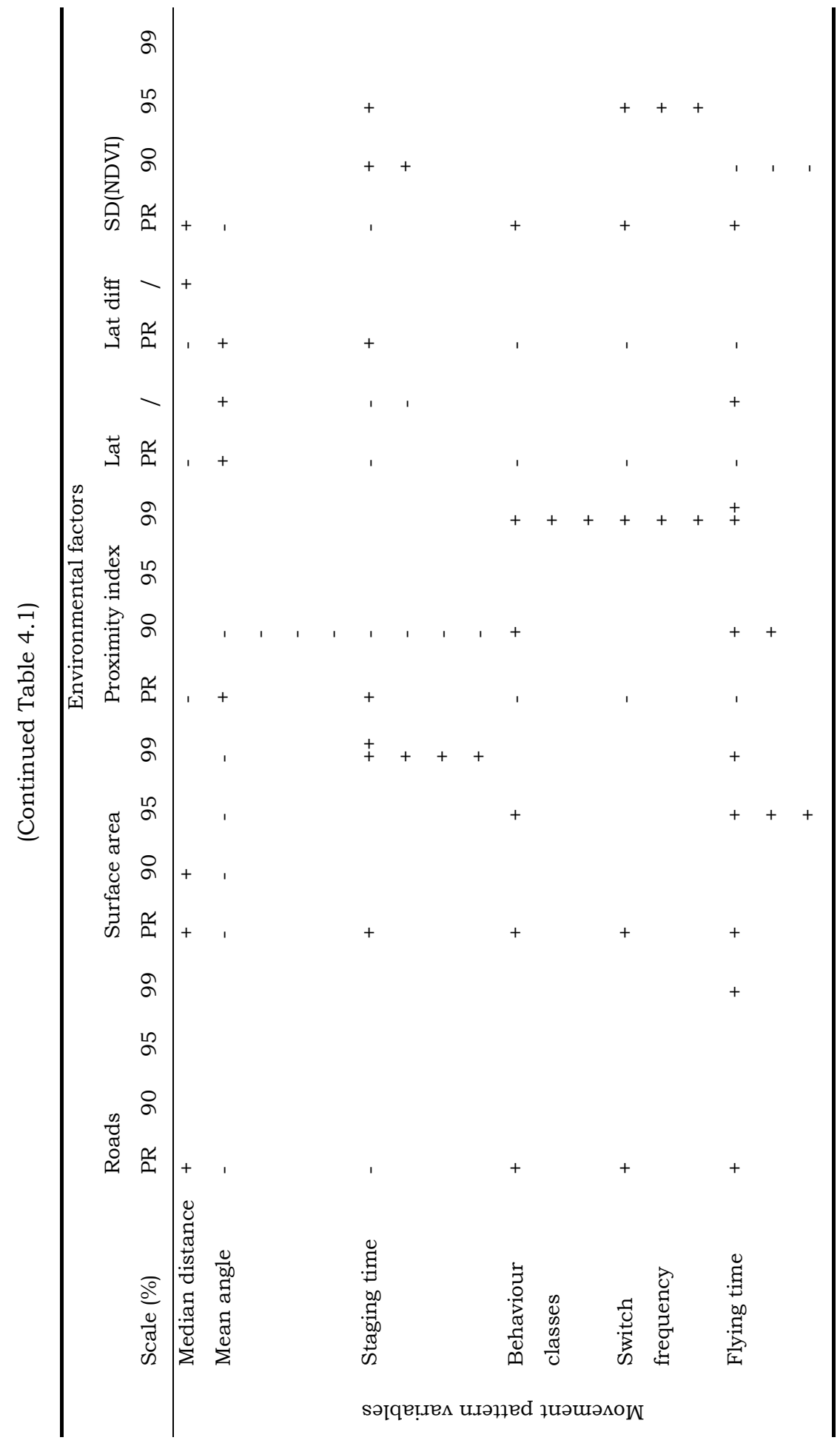




\section{Multivariate analysis}

The canonical axes were all significant at all areas (99\%, 95\%, and 90\%). High quality sites were expected to be found in the top right quarter of the graphs at $99 \%$ and $95 \%$ areas (Figure 4.3ab), and in the bottom right quarter at $90 \%$ area (Figure $4.3 \mathrm{c}$ ), associated with low median distance, low flying time, low frequency of behavioural switches, low number of behavioural classes, low mean angles, and with a higher total staging time. The percentage of agricultural lands and forests significantly explained the variation in movement patterns in different sites at all scales, whereas the percentage of water was only significant at the $95 \%$ and $99 \%$ scale. The percentage of agricultural lands was positively correlated with median distance, flying time, frequency of behavioural switches, and number of behavioural classes at all scales (Figure 4.3). Other factors positively associated with these movement patterns include road density, area of grasslands, and area of wetlands plus grasslands. Percentage of water and percentage of forests were both negatively correlated with these four movement variables. Total staging time and mean angle were best extracted on the second RDA axis and were not strongly correlated with the other movement variables. A higher percentage of agricultural lands and a higher percentage of wetlands were often related to a longer staging time in a site (Figure 4.3). 

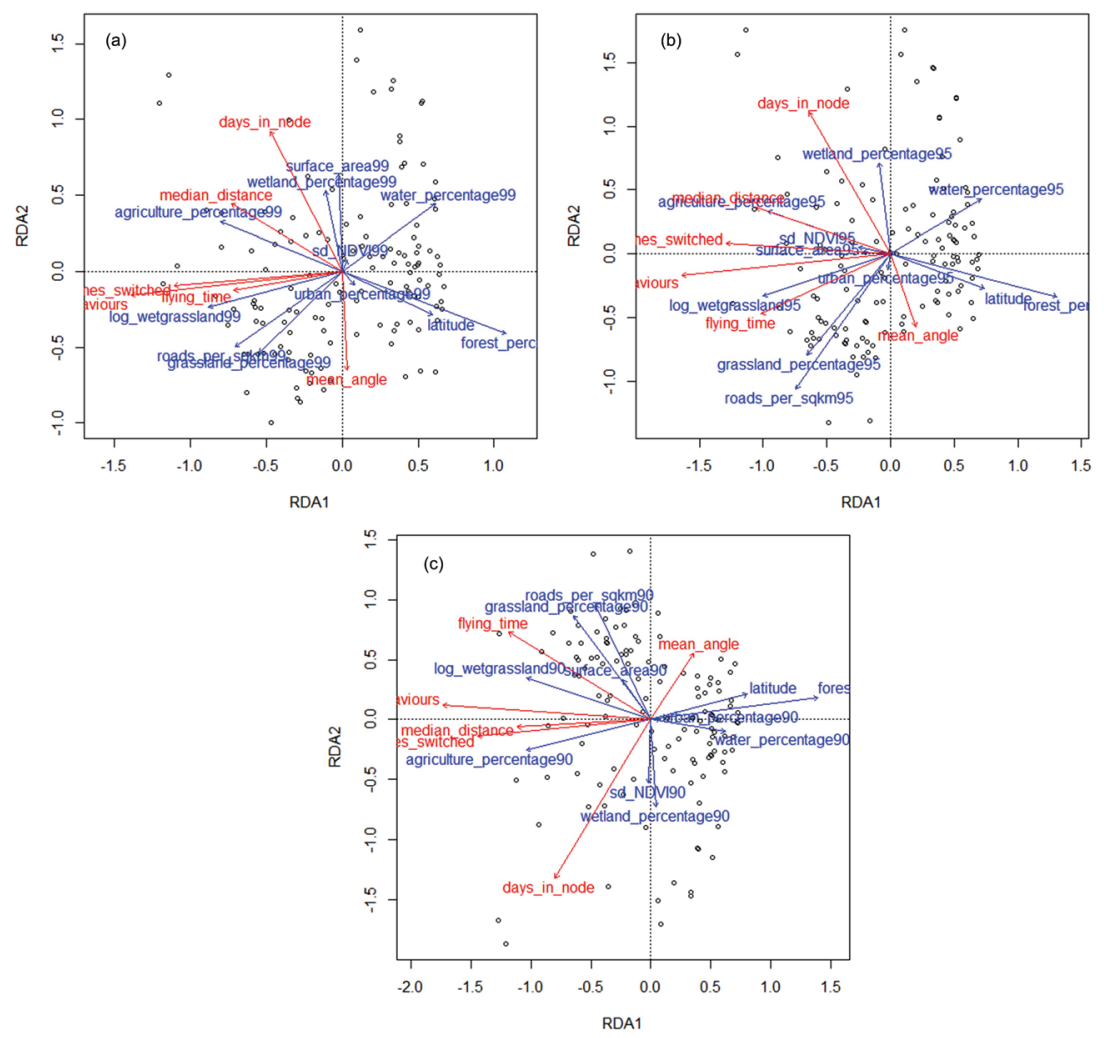

Figure 4.3 Triplots from the three Redundancy Analyses (RDA) at the 99\% (a), 95\% (b) and $90 \%$ (c) area. The red arrows are movement variables, blue arrows represent environmental factors. The circles are sites. The first axis explained $11 \%$ of the variation at the $99 \%$ and $95 \%$ scale, and $12 \%$ at the $90 \%$ scale, and the second axis explained $6 \%$ of the variation at all scales.

\subsection{Discussion}

East Asian geese respond negatively to human activities, but in a different way compared to geese that have their North-South migration flyway through Europe or North America. Generally, farming activities negatively affect the habitat quality for these greater white-fronted geese in the East Asian-Australasian Flyway, as they fly more, make longer movements, and switch their behaviour more frequently in a stopover site with a higher proportion of agricultural lands (Table 4.1). Other human activities, i.e., development of urban lands and roads, did however not strongly affect their movement. Differently, many waterfowl species in Europe and North America take use of the abundant food resources in farmlands, creating conflicts between farmers and wild birds 119 . 
Hence, East Asian waterfowl prefer foraging on natural habitats, and are more disturbed in agricultural lands in China 51 . This agrees with our results that the geese move more, and more frequently change their behaviour at stopover sites with a higher fraction of agricultural lands. Moreover, we found that geese expanded their movements (larger flight time and longer movement distances) in stopover sites with a higher water availability and a larger wetland proximity (Table 4.1). These natural land covers may be their major habitats, and a larger area and a higher connectivity increases movements of waterfowl 81,159 .

Hence, agriculture lands in China are not optimal habitats for waterfowl. On the one hand, these farmlands are highly disturbed by human activities from resident settlements (1.0\% are urban lands in these stopover sites), and hunting ${ }^{128}$. Migratory geese in East Asia therefore prefer to use habitats with a lower human density ${ }^{126}$. On the other hand, although $58 \%$ of the home range of the studied geese consists of farmlands, these agriculture lands may provide only limited food resources for waterfowl, as for example crops are frequently removed by free-ranging poultry from local farmers in non-breeding habitats in the Yangtze River Basin, Southeast China ${ }^{51}$. A higher disturbance level increases energy expenditure while a lower food availability reduces intake rate, and as a result, these stopover sites are less attractive for resting and refuelling compared to those with a lower cover of agriculture lands.

Water bodies and wetlands are the main habitats for East Asian waterfowl. However, these natural habitats in the East Asian-Australasian Flyway are, at present, degrading rapidly 35,169 . Firstly, build-up of dams affect water level, and subsequently disrupt the natural phenology of recessional grasslands ${ }^{127}$, which can result in a mismatch between the peak in forage availability and quality, and the arrival time of migratory waterfowl. Secondly, we found that a higher forest cover at stopover sites impeded goose movements indicated by a shorter median distance (Table 4.1). Wetland availability may decrease with expanding forest cover. Especially in Russia, staging sites became less suitable for geese because a large part of wetlands and grasslands have transformed to forest by economically driven land abandonment 68,123. Thirdly, although not so much reflected in our results, an increasing proximity to human disturbance, e.g., urban expansion, road construction, and illegal killing, can also reduce the quality of natural habitats for waterfowl 81,126 . 
Therefore, migratory waterfowl in the East Asian-Australasian Flyway seem seriously threatened by land use changes related to anthropogenic practices. Consequently, unlike in Europe and North America where waterfowl population sizes boosted in the last decades 49,185, East Asian waterfowl experienced a rapid population decline since 1950s 55,169. However, opening up farmlands for East Asian geese could also extend foraging habitats, and provide additional food resources for refuelling, as shown by European and North American studies. To improve habitat availability and quality for geese, it may be effective to provide agricultural subsidies to farmers to compensate for agricultural damage caused by geese 186. Besides, setting up or expanding natural reserves for conservation, especially in important stepping-stone sites for an efficient migration network ${ }^{170}$, can also protect sites from intensive human reclamation 187.

According to our results, the relationships between environmental factors and bird movement variables can be used to predict the quality of a stopover site. This enables scientists to simulate the effect of site removal from a migration network 37,188 , and also to generate early warning signals for network collapse. Our methodology to predict habitat quality is expected to be reproduceable for other bird and mammal species who move in human dominated landscapes. Environmental factors were best in predicting flying time and median distance at stopover sites (Figure 4.2; Appendix S4.1), and thus, movement patterns related to flying distance and time can yield good indicators of the quality of a stopover site for migratory waterfowl. Turning angles could be poorly predicted by anthropogenic nor ecological factors in our study (Appendix S4.2), and seem therefore not well suitable for predicting quality of stopover sites for waterfowl. This finding seems contrasting to the study of Aben et al. ${ }^{180}$ where the turning angle of forest birds was a good index for the encroachment of human dominant land use types. Future studies can improve this method, by using tracking data with a higher temporal resolution, which may show another explanatory role of turning angles. For more specific behavioural classes, such as foraging, resting, or walking, the tracking interval could be optimised to obtain the highest accuracy for the classification 189. 


\subsection{Acknowledgements}

We thank F. Xu, W. Zhang, J. Wei, B. Wielstra, G. Liu, H. Luo, S.

Balachandran, S.K. Behera, M. Ponnaian, Z. Lv, W. Wu, B. Gao, T. Zhang, and other members of the field crew for their supports in bird tracking. We thank J.A.J. Eikelboom for his insightful suggestions in movement analysis. Financial support was provided by the National Key R\&D Program of China (No. 2017YFA0604404), the National Natural Science Foundation of China (No. 41471347), and Chinese Scholarship Council (no. 201600090128). 


\section{Supplementary information}

Appendix S4.1-S4.5 display the predicted values for the movement pattern indices (i.e., median distance, mean angle, staging time, number of behavioural classes, the frequency of behavioural switches) in a site by the best model against the observed values of this variable. The figure for the flying time is Figure 4.2 in the main text.

This is a common legend for Appendix S4.1-S4.5: (a) the model with original dataset $(\mathrm{N}=90)$ which are the sites used to create the model, (b) the model with validation dataset $(\mathrm{N}=39)$ which are the sites that were randomly selected for validation, (c) the model with all data $(\mathrm{N}=129)$. The red lines indicate the $\mathrm{X}=\mathrm{Y}$ line (i.e. a perfect prediction), with the $\mathrm{R}^{2}$ value reflecting the accuracy of the model compared to this line.

Appendix S4.1 The predicted values for the log-transformed median distance in a site by the best model, plotted against the observed values of this variable.
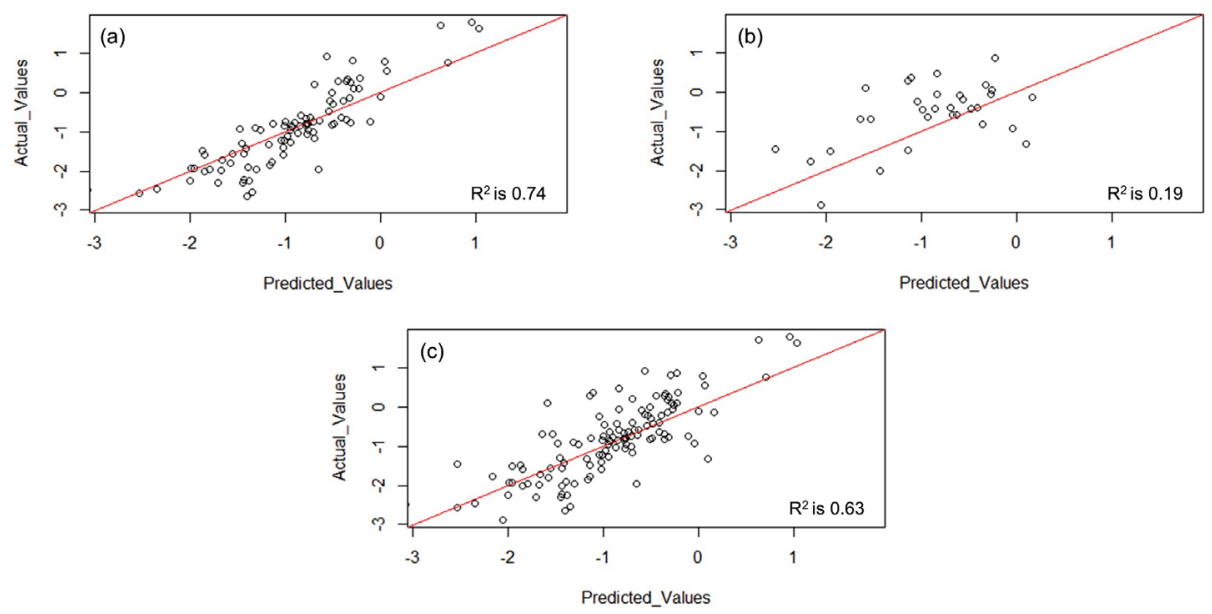
Appendix S4.2 The predicted values for mean angle in a site by the best model, plotted against the observed values of this variable.
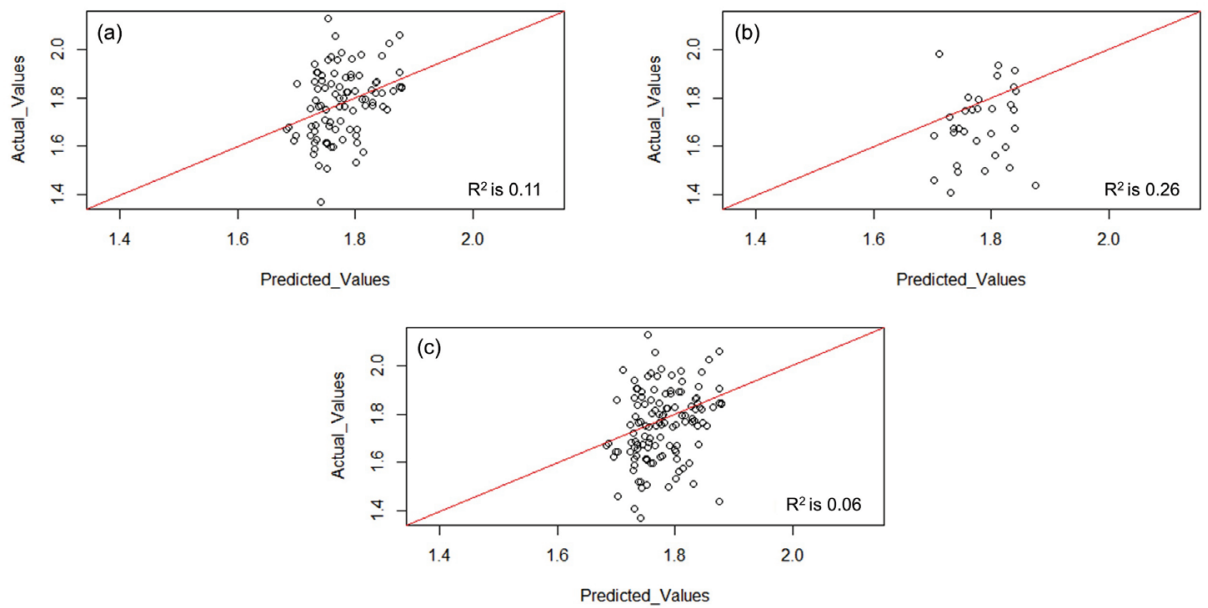

Appendix S4.3 The predicted values for the log-transformed staging time (in days) in a site by the best model, plotted against the observed values of this variable.
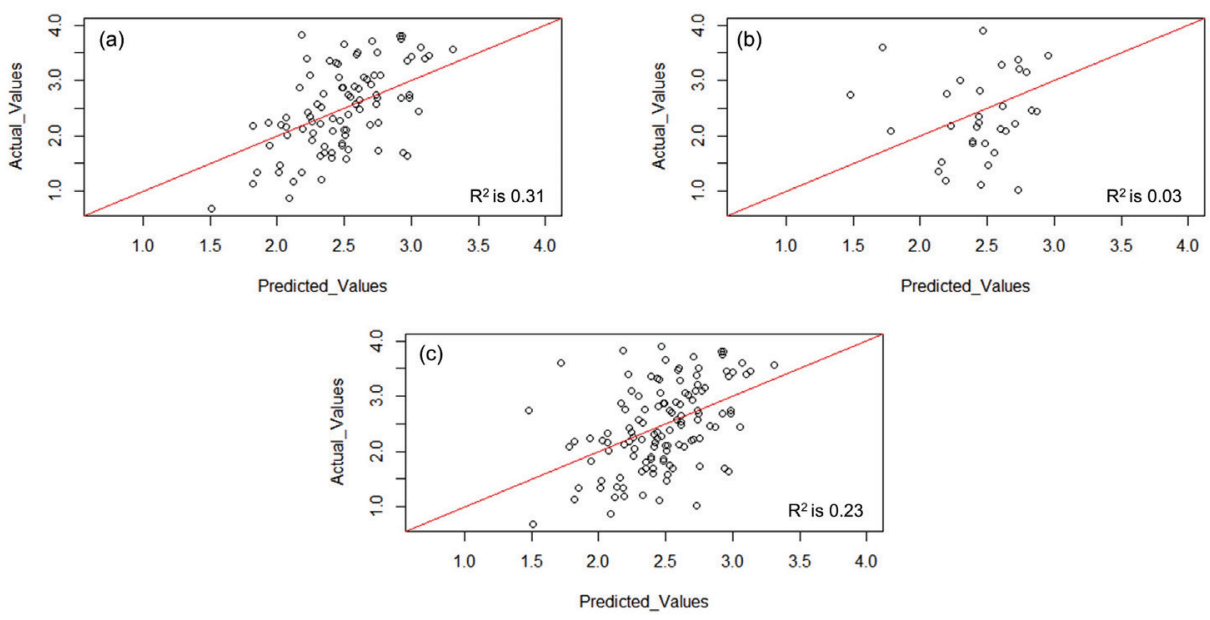
Appendix S4.4 The predicted values for the log-transformed number of behavioural classes in a site by the best model, plotted against the observed values of this variable.
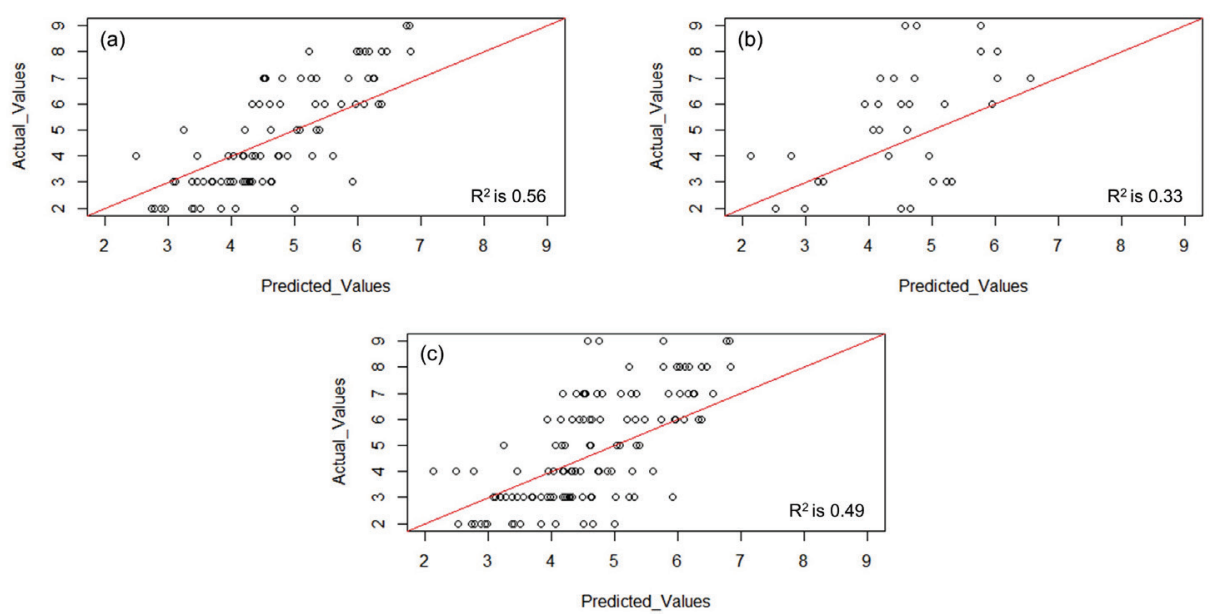

Appendix S4.5. The predicted values for the log-transformed frequency of behavioural switches in a site by the best model, plotted against the observed values of this variable.
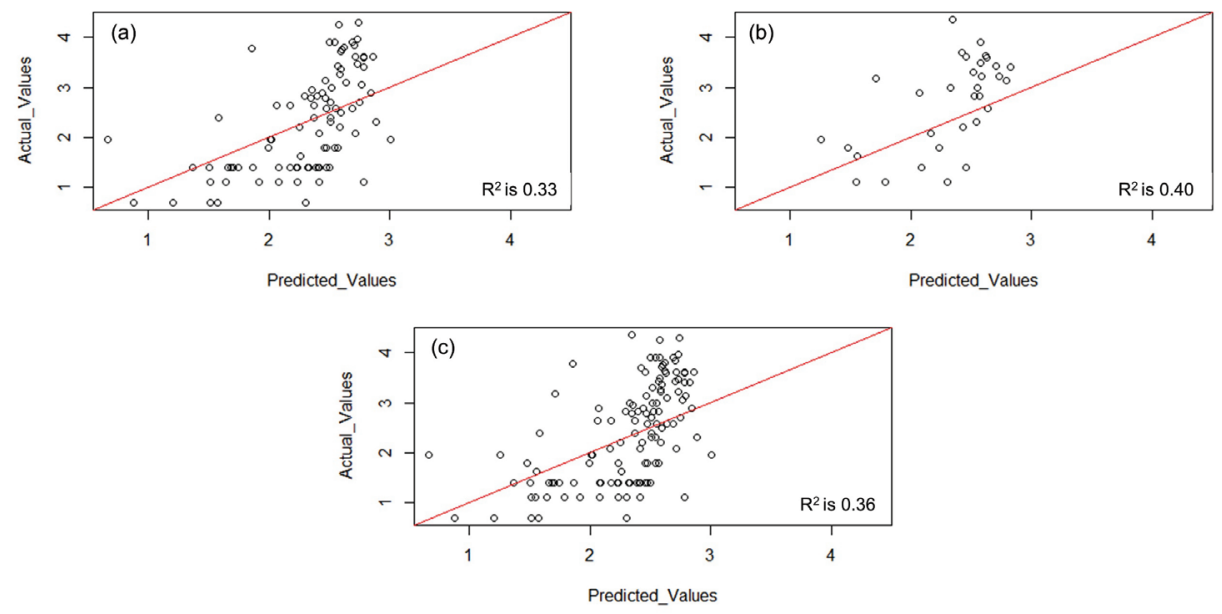
Indicators of site loss from a migration network: anthropogenic factors influence waterfowl movement patterns at stopover sites 
CHAPTER 5 


\section{A network approach to prioritize conservation efforts for migratory birds}

Xu Y., Si Y., Takekawa J. Y., Liu Q., Prins H. H. T., Yin S., Prosser D. J., Gong P., de Boer W. F. A network approach to prioritize conservation efforts for migratory birds. Conservation Biology 34, 416-426 (2019) 


\section{Abstract}

Habitat loss can trigger migration network collapse by isolating migratory birds' breeding grounds from non-breeding grounds. Theoretically, habitat loss can have vastly different impacts depending on the site's importance within the migratory corridor. However, migration network connectivity and the impacts of site loss are not completely understood. Here we used GPS tracking data from four bird species in the Asian flyways to construct migration networks, and proposed a framework for assessing network connectivity for migratory species. We used a node removal process to identify stopover sites with the highest impact on connectivity. In general, migration networks with fewer stopover sites were more vulnerable to habitat loss. Node removal in order from the highest to lowest degree of habitat loss yielded an increase of network resistance similar to random removal. In contrast, resistance increased more rapidly when removing nodes in order from the highest to lowest betweenness value. We quantified the risk of migration network collapse and identified crucial sites by first selecting sites with large contributions to network connectivity, and then identifying which of those sites were likely to be removed from the network (i.e., sites experiencing habitat loss). Among these crucial sites, $42 \%$ were not designated as protected areas. Setting priorities for site protection should account for the site's position within the migration network, rather than only site-specific characteristics. Our framework for assessing migration network connectivity enables site prioritization for conservation of migratory species. 


\subsection{Introduction}

In recent years, the populations of many migratory species have rapidly declined due to loss and degradation of suitable habitats, caused by rapid economic development, intensive human disturbance, and inefficient conservation policies, 35,55,88. The majority of migratory birds are not effectively protected across their migration network 94,190 . For instance, the swan goose (Anser cygnoides) is categorized as vulnerable, but is substantially threatened by high levels of hunting and wetland conversion 150. Additionally, the greater white-fronted goose (Anser albifrons), barheaded goose (Anser indicus), and whooper swan (Cygnus cygnus) are widespread and abundant in the wild, and are categorized as least concern species, but their populations have rapidly declined in many areas, indicating inadequate conservation efforts for these migratory birds 55,150 .

Wetland degradation and loss can weaken the integrity of the migration networks of individual species, or even promote migration network collapse, by isolating birds' breeding grounds from their wintering grounds 37 . Moreover, degradation and loss of stepping-stone nodes from a habitat network may limit a species' ability to shift ranges, which is an important strategy used by migratory birds to cope with environmental changes ${ }^{57}$. To better understand how environmental changes impact existing migration networks and to guide targeted conservation measures, it is important to evaluate a migration network's connectivity and resilience, and to identify crucial sites that might trigger network collapse.

In conservation policy-making, a site's importance for migratory species is often evaluated in terms of the presence of suitable habitat, habitat vulnerability, degree of habitat loss, protection status, and/or species abundance or diversity 44,81,191-193. Although such evaluation is straightforward, it does not account for relationships among different sites or the site's context within a network, e.g., the availability of alternative sites along the migration flyway 44,194 . Previous studies demonstrate that network-level metrics (e.g., habitat centrality) are more suitable for evaluating habitat importance for species with a movement pattern, and should thus be included in management decisions 190,195,196. Recent resolutions emphasize the importance of considering ecological networks and the connectivity of migratory species when addressing conservation of migratory species 197. 
Theoretically, the same degree of habitat loss from sites at different network locations could have completely different impacts on migratory birds, ranging from not affecting the population size at all to causing rapid extirpation of the species ${ }^{93,94}$. For instance, rapid population decline has occurred among migratory birds with higher reliance on stopover sites in the Yellow Sea region ${ }^{35}$, which may be abundant in resources providing energy reserves for subsequent migration 198 or located in a critical position connecting breeding and non-breeding sites ${ }^{37}$. Thus, in this case, population decline may not be stopped by implementing conservation measures elsewhere ${ }^{94}$.

Since bird migration is a directed event that occurs at continental or cross-continent scales, setting priorities for conservation efforts requires an integrated evaluation considering both habitat availability and the connectivity of sites along migration routes. Network theory is useful for such evaluations. Most studies investigating complete bird migration networks are based on theoretical investigations of conceptual site configurations $73,93,199-201$. To prioritize conservation efforts for specific sites, it is pivotal to combine node removal scenarios and habitat loss patterns in migration networks that are empirically defined by sites exhibiting seasonal bird occupation.

Merken, et al. ${ }^{44}$ analysed the migration networks along the Black SeaMediterranean Flyway across the Sahara, revealing that the trans-Sahara migration flyway for waterbirds was well-connected. Crucial sites in this migration network were identified by quantifying the importance of each involved wetland. Shimazaki, et al. ${ }^{37}$ analysed migration networks of the oriental white stork (Ciconia boyciana), and determined potential collapse risks by simulating the removal of important stopover sites. They demonstrated that the storks would be unable to reach their wintering sites along the Yangtze River if they lost stopovers in the Bohai Bay during southward migration. Nicol, et al. 202,Iwamura, et al. ${ }^{203}$ simulated population flows in shorebird migration networks upon sea level rise, and thereby provided insightful algorithms of population flows within these migration networks, for developing efficient conservation strategies. Additional information is needed regarding the extent to which site-specific variables (e.g., habitat loss), and network metrics that characterize a node with regard to its network position, contribute to migration network breakdown. 
In the present study, we aimed to investigate how migration network connectivity was impacted by site-specific habitat loss and degradation compared with node-specific network metrics. Using high-resolution GPS tracking data, we quantified migration network connectivity for large-bodied waterfowl species in the Central and East Asian-Australasian Flyways. We quantified the importance of each stopover site based on its contribution to the network's resistance relative to bird migration. These data, together with the degree of habitat loss and the protection status of these sites, were used to identify sites for which conservation efforts should be prioritized. Our analytical framework compared the effects of site-specific metrics on the migration networks' connectivity, thus guiding priority setting for site conservation.

\subsection{Methods}

\section{Data}

A total of 81 swan geese, 54 greater white-fronted geese, 93 bar-headed geese, and 10 whooper swans were tagged with GPS loggers in the East Asian-Australasian Flyway and Central Asian Flyway, between 2005 and 2018, yielding 63 full tracks of their northward migration and 108 full tracks of southward migration (Figure 5.1a; Appendices S5.1 \& S5.2). The loggers were programmed to record 6-12 GPS locations (latitude and longitude) per day for each individual. However, GPS records were sometimes missing due to low battery levels or satellite acquisition failure. Detailed capture and deployment methods are provided in Appendix S2 and in prior publications (Batbayar, et al. ${ }^{90}$, Newman, et al. ${ }^{204}$, Si, et al. ${ }^{89}$, and $\mathrm{Xu}$ et al. 2019c).

To quantify the degree of habitat loss for each site in migration networks of the focal species, we obtained land cover data for 1992 and 2015 from the European Space Agency (ESA) CCI 300-m annual global land cover products (esa-landcover-cci.org). We quantified wetland loss between 1992-2015 by extracting the area of water and grassland from the maps of these years. Habitat loss calculation did not include changes in croplands. While American and European goose populations benefit from agriculture expansion, massive wetland conversion to agricultural lands has a negative impact on most waterfowl species in eastern Asia 51,89 188. 

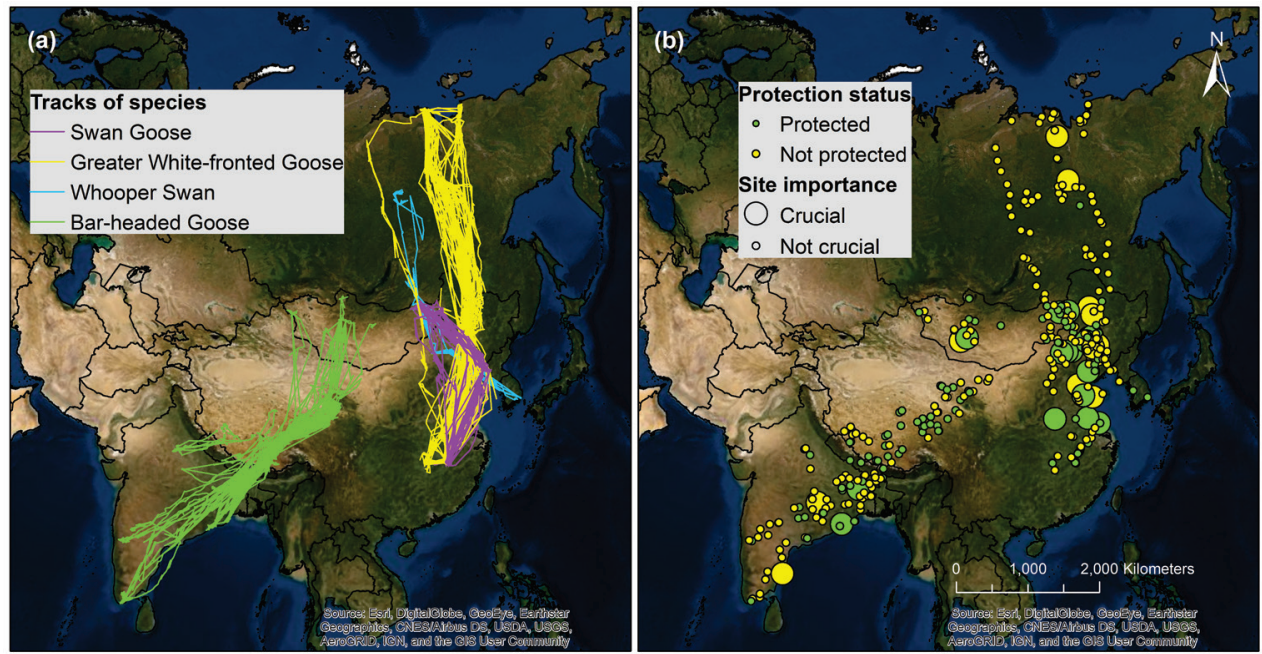

Figure 5.1 Satellite tracks and breeding, non-breeding, and stopover sites of swan geese, greater white-fronted geese, whooper swans, and bar-headed geese. A site was deemed non-protected (yellow) when it contained no designated protected area.

Crucial sites (large circles) were defined as having a normalized betweenness of $\geq 10 \%$ and habitat loss (mean, $7.8 \%$; 95\% CI, $\pm 4.1 \%$ ).

\section{Utilized sites and migration lags}

We identified the breeding, wintering, and stopover sites of each individual bird using a Dynamic Brownian Bridge Movement Model (dBBMM) ${ }^{182}$. Utilization distributions were derived at a $10 \times 10 \mathrm{~km}$ resolution, for the annual northward and southward migration of each tracked bird. Based on visual inspection of the tracking data, we used a window size of 11 locations, and margin size of 3 locations ${ }^{182}$. Geographical ranges of $90 \%$ isopleths of the utilization distributions (i.e., highly utilized areas with short flights) ${ }^{89}$ ) were defined as the sites utilized in northward and southward migrations (i.e., breeding, non-breeding, and stopover sites) based on visual inspection. We included sites that birds used for $\geq 2$ days, considering that a site should be used for at least $48 \mathrm{~h}$ for settling and refuelling ${ }^{205}$. To measure the effects of sample size, we conducted a sensitivity analysis of the effect of the number of southward tracks of swan geese and bar-headed geese on site detection (Appendix S5.3).

We defined migration lag as the non-stop flight distance from one site to the next. Distances between the boundaries of utilized sites were calculated under Azimuthal equidistant projection. Tracks missing data for over two weeks were excluded from distance calculations. We calculated the maximum and median migration lags from the tracking data per season, per species. 


\section{Migration networks}

The breeding, wintering, and stopover sites for each individual bird were defined as nodes in the migration network. When the distance between two nodes was shorter than the maximum migration lag, these nodes were connected. Due to seasonal directionality, only low-latitude to high-latitude sites were connected in northward migration networks, and only highlatitude to low-latitude sites were connected in southward migration networks. We assumed that greater site-to-site distance was associated with increased cost of movements between sites. Thus, the weight of each site-tosite connection was defined by a between-sites dispersal probability, calculated as the cost of moving between two sites with a decreasing exponential function (equation 5.1) ${ }^{146}$. This probability function assumed that a greater distance between two sites correlated with a lower probability that migratory birds would move from one site to the next. For simplicity, this assumption was based only on energetic expenditures, without considering differences in searching and settling costs, forage abundance and quality, or predation risk 208 . Based on these nodes and weighted connections, we constructed northward and southward migration networks for each species.

$$
P_{i j}=e^{-k d_{i j}}
$$

In equation $1, P_{i j}$ is the dispersal probability between sites $i$ and $j, d_{i j}$ is the edge-to-edge distance between sites $i$ and $j$; and $k$ is a constant defined by the migration lags of the tracked species. We set $k$ to obtain a dispersal probability of $50 \%$ when $d_{i j}$ equalled the median migration lag of the focal species.

\section{Network metrics}

To identify important stepping-stone sites, we calculated network metrics related to node centrality, measuring the site's importance-i.e., betweenness, weighted degree, and node resistance. We quantified each metric's importance by comparing its contribution to network connectivity (quantified by effective resistance), using a site removal process with removal order determined by betweenness, node degree, node resistance, or degree of habitat loss. Since our main focus was identifying important stepping-stone sites connecting breeding and non-breeding sites, the breeding and non-breeding sites were not included in the site removal process. 
For each pair of sites in the migration network, we identified the shortest path, having the minimum weighted path length between the two nodes, measured by the Dijkstra algorithm ${ }^{214}$. Node betweenness was quantified by the number of shortest paths passing through that node ${ }^{14,15}$, and was calculated using the second-generation weighted betweenness measure ${ }^{4}$. Node betweenness was normalized by dividing it by the highest betweenness value. Node degree indicates the connection strength between the focal site and other sites in the network, and was measured as the sum of weights of the connections to and from the focal node, calculated with the Dijkstra algorithm ${ }^{214}$. We measured node resistance by the effective resistance 206 between the focal node (i.e., a stopover site) and the breeding site, plus that between the focal node and the non-breeding site. Node resistance indicates the resistance for travelling between the focal stopover site to the breeding site and the non-breeding site. We also calculated the degree of habitat loss at each stopover site as the ratio (\%) of habitat loss/gain between 1992 and 2015. We selected 1992 as a baseline due to the rapid urbanization and socioeconomic development in East Asian countries since 1992 118,188. The 1992 data is the earliest land cover map in the analysed dataset.

We calculated the metrics for all sites of the initial networks, and then removed the sites one by one, under five scenarios: highest to lowest relative betweenness, highest to lowest weighted degree, lowest to highest node resistance, highest to lowest degree of habitat loss, and 99 sequences of random removal (each observed network comprised <99 sites). Effective resistance reflected the connectivity between breeding and non-breeding sites by accounting for both migration cost and alternative routes ${ }^{206}$. After each removal, we calculated the network's effective resistance, and we compared the speed of effective resistance increase under different site removal scenarios.

To select the best metric for defining crucial network sites, we compared the effect index of node removal ( $E_{m}$; equation 5.2 ) using different network metrics ( $m=$ betweenness/degree/node resistance).

$$
E_{m n}=\frac{N_{0} \times \ln \left(\frac{R_{m n}}{R R_{n}}\right)}{N_{c}}
$$

In equation 5.2, $R R_{n}$ is the effective resistance when n nodes were removed at random, $R_{m n}$ is the effective resistance when $\mathrm{n}$ nodes were removed in the sequence of metric $m, N_{O}$ is the total number of nodes in the original 
network, and $N_{c}$ is the number of nodes removed upon network collapse. As $E_{m}$ was not normally distributed (Kolmogorov-Smirnov test, $D=0.24, p<$ 0.001), we used a Kruskal-Wallis test followed by a non-parametric multiple comparison test to analyze differences in the effects of different metrics ( $\left.E_{\text {betweenness }}, E_{\text {degree}}, E_{\text {node resistance }}\right)$. The metric with a significantly higher $E_{m}$ level $(p \leq 0.05)$ was used to define a crucial stopover site. We also tested between-metrics differences for each network separately to show species differences (Appendix S5.9).

To define whether a site was protected, we overlapped the map of protected areas with the ranges of sites in the migration networks. Some Chinese protected areas were missing from the international dataset; thus, we merged the polygon map from the World Database of Protected Areas (WDPA; accessed on 06-04-2018 at protectedplanet.net) with the national protected areas in China and the site network of the East AsianAustralasian Flyway Partnership (EAAFP; accessed on 20-05-2019 at eaaflyway.net). When a site overlapped with the map of protected areas, we defined it as protected. Otherwise it was considered not protected. These calculations were performed in ArcMap 10.2.1 under the cylindrical equal area projection.

\subsection{Results}

\section{Migration patterns}

Swan geese, greater white-fronted geese, whooper swans, and bar-headed geese, respectively, exhibited northward migration networks comprising 23, 72,15 , and 81 sites, and southward migration networks comprising 45, 27, 13, and 67 sites. The small sample size of whooper swans yielded an artificially small number of migration network nodes (Appendix S5.3); therefore, whooper swan results were included only as an illustrative example of small networks. Among all tracked birds, the median distance between sites was $203 \mathrm{~km}$ in both northward and southward migration. The maximum migration lag (travel distance between sites) was $3180 \mathrm{~km}$ for southward migration, and $3018 \mathrm{~km}$ for northward migration (Appendix S5.5). 

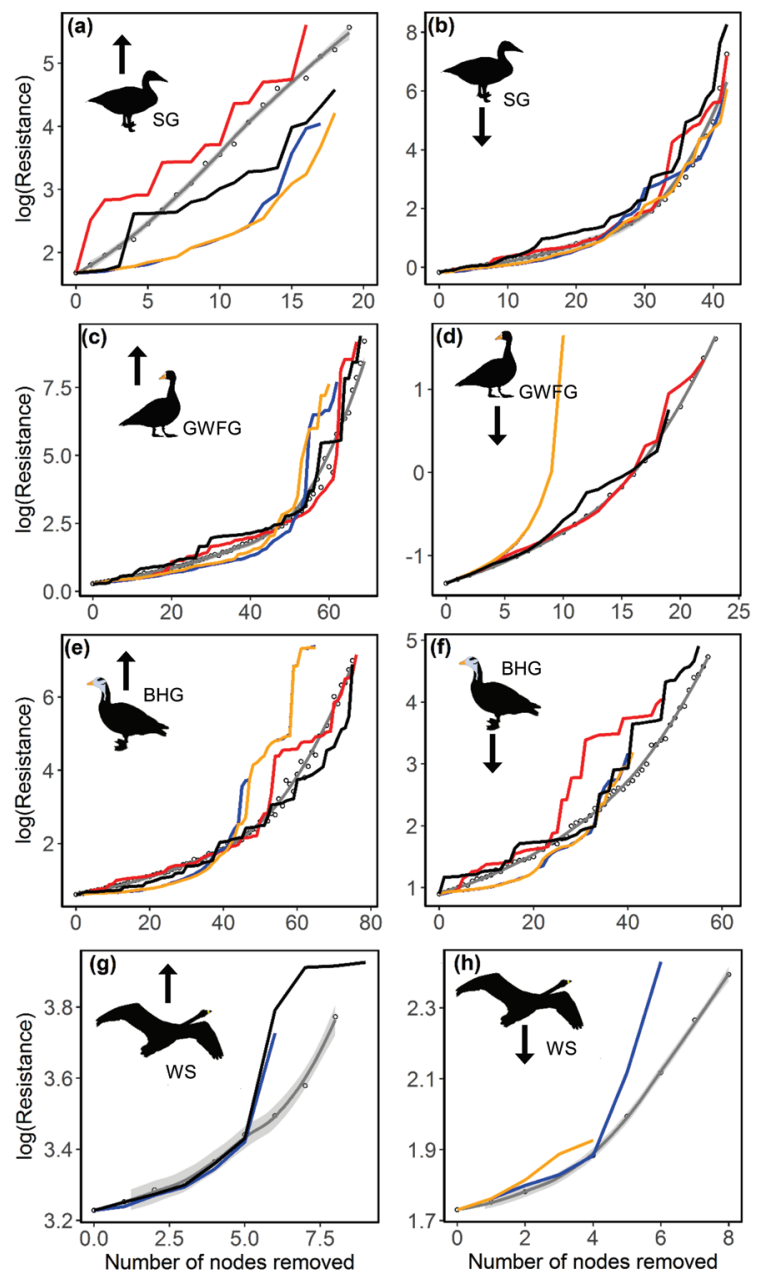

Metrics - Betweenness- Degree - Node Resistance- Habitat Loss

Figure 5.2 Changes in the effective resistance of migration networks upon cumulative removal of stopover sites. Grey lines and dots show the patterns of the migration networks' effective resistance upon random site removal. The other line colours show the changes of effective resistance upon site removal in the order of

their degree of habitat loss (black), betweenness (red), degree (blue), and node resistance (yellow). Panels a, $\mathbf{c}, \mathbf{e}$, and $\mathbf{g}$ indicate the northward migration networks (arrow pointing up) of swan geese (SG), greater white-fronted geese (GWFG), barheaded geese (BHG), and whooper swans (WS), respectively. Panels $\mathbf{b}, \mathbf{d}, \mathbf{f}$, and $\mathbf{h}$ indicate the southward migration networks (arrow pointing down) of swan geese, greater white-fronted geese, bar-headed geese, and whooper swans, respectively. Upon network collapse, the effective resistance became infinity, such that the end of each line represents the point at which the network collapsed. Due to the small number of included whooper swans, their data were only used as an example illustrating how a small network behaves. Their small networks were vulnerable, since they collapse if just a few nodes (stopover sites) become unusable. 


\section{Site removal}

In general, the migration networks' effective resistance slowly increased at the beginning of node removal, rising with increasing removal of nodes (Figure 5.2b,c,e,f). However, the effective resistance increase was rapid at the start of node removal in migration networks comprising relatively low numbers of sites-i.e., greater white-fronted geese (southward), swan geese (northward), and whooper swans (both directions; Figure 5.2a,d,g,h).

Compared with random site removal, the migration networks' effective resistance generally increased faster when sites were removed in order of increasing betweenness. Small networks (whooper swans) collapsed quickly upon removal of the site with highest betweenness (Figure 5.2g,h). However, in the southward migration network of greater white-fronted geese, the effective resistance increased faster when sites were removed in order of decreasing node resistance or degree (Figure 5.2d; Appendix S5.9). For most networks, site removal in order of degree of habitat loss yielded an effective resistance increase similar to random site removal. However, in the southward migration network of swan geese, effective resistance increased more rapidly upon site removal in order of habitat loss compared to all other removal orders (Figure 5.2b). The northward migration network of swan geese showed the opposite pattern, with random site removal yielding a more rapid increase in effective resistance.

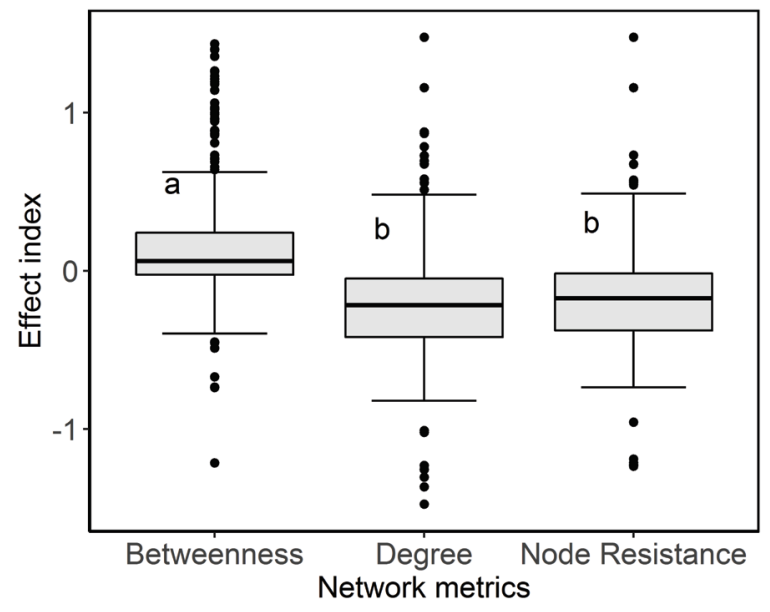

Figure 5.3 Differences in the effect indices of betweenness, degree, and node resistance. The letters on the boxes represent identical groups as determined by the multiple comparison test at $p=0.05$. Betweenness had a significantly higher effect index than the other metrics. 


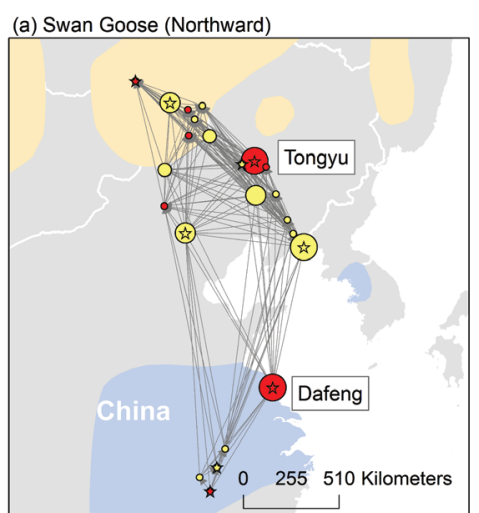

(c) Greater White-fronted Goose (Northward)

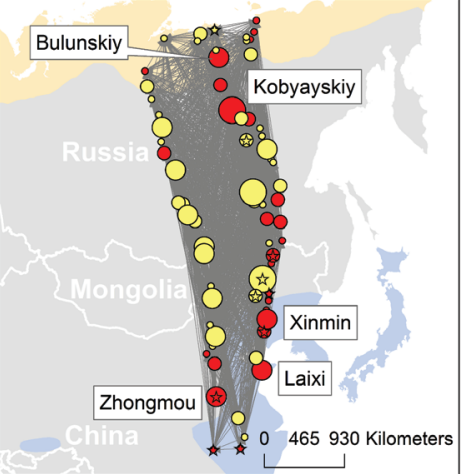

(e) Bar-headed Goose (Northward)

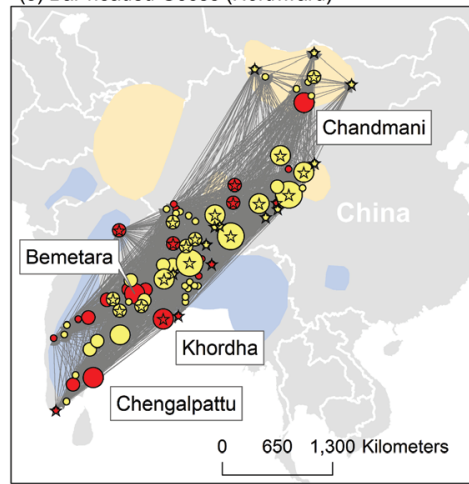

(b) Swan Goose (Southward)

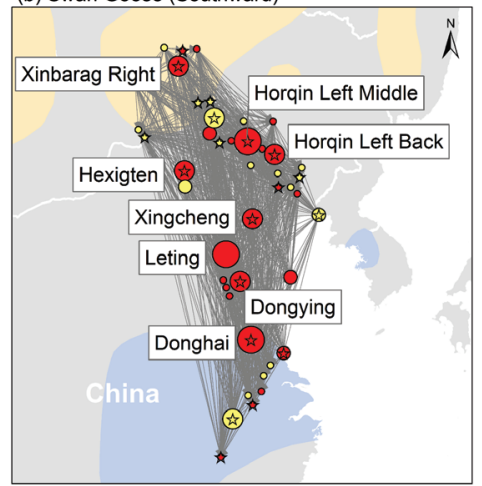

(d) Greater White-fronted Goose (Southward)

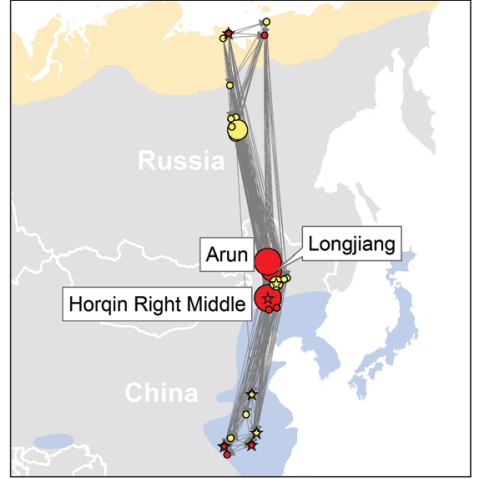

(f) Bar-headed Goose (Southward)

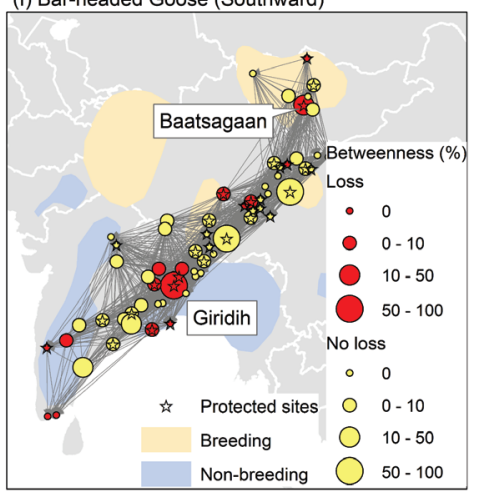

Figure 5.4 Migration networks and locations of crucial stopover sites. The betweenness of each stopover was normalized via division by the highest betweenness value in the corresponding network. Circle size indicates the normalized betweenness. Red circles indicate sites exhibiting habitat loss between 1992-2015. Yellow circles indicate sites without habitat loss. Sites were deemed crucial stopover sites if they had a normalized betweenness of $\geq 10 \%$ and habitat

loss (mean, $7.8 \%$; 95\% CI, $\pm 4.1 \%$ ). Crucial sites are identified with the corresponding administrative name (i.e., county in China, district in Russia, sum in Mongolia, and taluk in India). Protected sites are marked with stars. We obtained species' breeding and non-breeding ranges from bird distribution maps of the world (v5.0) produced by BirdLife International (birdlife.org). 


\section{Site importance}

Effect indices significantly differed between different network metrics (Kruskal-Wallis test, $X^{2}=98.0, d f=2, p<0.001$ ). Betweenness had a significantly higher effect index than degree and node resistance (Figure 5.3). Therefore, we defined a site's importance for maintaining network connectivity based on betweenness. Sites were defined as crucial if they showed high relative betweenness $(\geq 10 \%)$ and were also likely to be removed from the network, i.e., experiencing habitat loss (mean $\pm 95 \%$ CI, $7.8 \% \pm$ 4.1\%) (Figure 5.4).

We identified 24 crucial sites, located in China ( $\mathrm{N}=16)$, India (4), Mongolia (2), and Russia (2). The sites in the Northeast China Plain (i.e., Tongyu, Xingcheng, Horqin Left Back banner, Hexigten, Horqin Left Middle banner, Xinmin, Horqin Right Middle banner, Longjiang, and Linxi) played important roles in the migration networks of swan geese and greater whitefronted geese. Of the 24 crucial sites, 8 were located in coastal regions of China (Dafeng, Donghai, Leting, Dongying, Xingcheng, and Laixi) or India (Chengalpattu and Khordha). Among sites with high betweenness in the southward migration network of swan geese, $80 \%$ showed habitat loss (1.5$40.0 \%$ ), and $50 \%$ of the crucial sites were in the coastal region of China. Among all stopover sites, $66 \%$ were not protected, and 10 crucial sites were not designated as protected areas (Figure 5.1b; Appendix S5.10).

\subsection{Discussion}

A well-connected migration network of well-protected sites can decrease migration costs and risks, thus facilitating bird migratory movements and increasing migration success ${ }^{44}$. Species that occupy large and robust migration networks have more alternative efficient routes, and are thus better able to cope with natural and human-driven environmental changes 188. The migration network structure of waterfowl in the East AsianAustralasian Flyway partly explains the population size fluctuations of these migratory birds, as population sizes decrease with losses of migration network functional connectivity ${ }^{169}$. In our present study, we quantitatively evaluated the robustness of migratory birds' migration network, and identified crucial stopover sites in terms of contribution to network connectivity and degree of habitat loss. We strongly recommend that regional policy makers apply our analytical framework when establishing conservation priorities, to decrease the risk of migration network collapse and to monitor policy implementation by local authorities. 
Many studies have examined the importance of nodes within a network, and multiple indices have been proposed for quantifying the contributions of individual nodes towards network connectivity 4,15,207. However, when identifying crucial sites, the utilized index should reflect their contributions to migration network connectivity (e.g., betweenness) as well as account for the ecological processes and mechanisms. Theoretical works have constructed simplified full-annual-cycle models for bird migration, to investigate bird population dynamics under habitat loss 73,93,199. However, these theoretical models do not consider the complexities of the spatial configurations of species-specific migration networks, such as differences in flyway broadness, migration distances, sizes of breeding ranges relative to non-breeding ones, and alternative migration routes 56,92,188. Our present model accounted for these features, and successfully defined crucial stopover sites in existing networks. This may be crucially important for enabling successful migration based on the empirical configuration of species-specific migration networks. Further research should be performed using our framework, modifying the algorithms and assumptions that define the probability of between-site movements. Here we only accounted for upper limits of non-stop flights, energetic terms, and migratory directions, but various other factors may also influence the cost of long-distance flights 208 , such as increased predation risk or disturbance, and the costs of searching and settling when making multiple stopovers. When sample sizes permit, it is interesting to empirically estimate the probability of travelling different distances based on tracking data 190,209.

Comparing the effects of node removal in the order of different metrics revealed betweenness as the most important factor identifying important stepping-stones. Betweenness measures the importance of a site in facilitating movement throughout the network ${ }^{207}$, and removing nodes with high betweenness rapidly disconnects a network ${ }^{210}$. Sites having high betweenness are necessary steps in multiple least-cost paths, such that their removal may force migrants to take suboptimal paths. Removal or degradation of sites with high betweenness can impede successful migration. This has also been reported for some forest bird species, whose mobility for range shift and long-distance dispersal can be sharply reduced by the loss of one critical stepping-stone site 57 . Further studies should analyze the availability and quality of the currently unused suboptimal alternative paths, which may serve as new migration routes, potentially preventing network collapse. 
Other network metrics, i.e., node resistance and degree, may also play important roles in identifying pivotal stepping-stones in migration networks for some species. We found that node resistance and degree outperformed betweenness in the southward migration network of greater white-fronted geese. However, in some smaller networks (e.g., the northward migration network of whooper swans), node removal in the orders of node resistance and degree reduced network connectivity more slowly than random removal. Therefore, we recommend that these metrics should not be used without comparison with betweenness, which is a more general metric that can be applied to designating prioritized conservation efforts for migration networks of various species.

The removal of sites with large habitat loss did not increase the effective resistance more than random site removal, suggesting that migration networks were resistant to patterns of habitat loss (from 1992-2015). Habitat loss was occurring in only some of the critical sites with a high contribution to network connectivity. However, removal of only a few sites with high betweenness can rapidly decrease network connectivity and trigger sudden collapse. These results are in agreement with theoretical simulations showing that heavy habitat loss at random sites has a lower impact on migratory species than a small amount of habitat loss at critical sites ${ }^{94}$. Loss of specific sites in a migration network-e.g., Bohai Bay in eastern China 37 and the Yellow Sea tidal mudflat 35 - can potentially isolate breeding from non-breeding sites, and/or trigger rapid population declines in migratory birds ${ }^{169}$. Therefore, the selection of crucial conservation areas for migratory species must account for both the severity of habitat degradation and the site's context within the species' network.

Migration network connectivity rapidly decreased when a network comprised a small number of sites. Networks are stable when migratory birds have plentiful alternative sites, but become more vulnerable following successive loss of sites. Unfortunately, some forms of habitat loss cannot be detected by land cover classification, e.g., water pollution and illegal killing. However, the presently detected habitat loss pattern was in agreement with previous studies showing that coastal regions and inland natural wetlands in eastern China have exhibited severe habitat loss due to rapid urbanization and sea-level rise 188,211. We also demonstrated that other sites in the migration networks were gaining habitat area, for example, in western China, as artificial wetlands were created in the forms of fish farms and 
reservoirs ${ }^{211}$. These increased alternatives can improve migration movement flexibility, boost network resilience, and subsequently mitigate population declines of migratory birds upon environmental changes 42,56. Overall, preventing habitat degradation and adding artificial habitats are essential for preventing migration network collapse, especially in locations with high betweenness.

Since we investigated identical network structures for different species, our results quantified the conservation needs of certain species and corresponding sites. Apart from the migration networks of whooper swans, which might be biased by the small sample size (Appendix S5.3), the most vulnerable analysed migration network was that of swan geese. The swan goose is categorized as a vulnerable species with relatively small population sizes and limited distribution ${ }^{150}$, and this species has already lost habitat area in important stepping stones in its networks over the past two decades. Its network integrity is impaired to the extent that it is now close to collapsing, and holds fewer alternative routes compared to a random network. As shown in Fig. 2, compared to their southward migration network, the northward migration network of swan geese is even more vulnerable to collapse, as the loss of only 14 sites will lead to network collapse. The Northeast China Plain and coastal regions in China contain critical sites $35,81,89$, many of which are currently unprotected (Figure $5.1 \mathrm{~b}$ ). This highlights the need for urgent conservation efforts at the province level, as protection at lower administrative levels reportedly has little or no effect 145. While the population numbers of greater white-fronted geese are declining in the East Asian-Australasian Flyway ${ }^{145}$, the numbers of barheaded geese in the Central Asian Flyway may actually increase. Our network integrity analysis appears to indicate that their migratory networks are sufficiently robust (Figure 5.2). However, concerns are raised by the rapid breakdown of the southward migration network of greater whitefronted geese upon node removal in the order of decreasing node resistance.

In summary, here we performed network analyses to guide recommendations for making conservation decisions regarding migratory species. Our results provide compelling evidence that destroying stopover sites with high betweenness values rapidly reduced migration network connectivity. Node resistance and degree were also important metrics for specific networks, i.e., the southward migration network for greater whitefronted geese. Our present analysis was based on tracked individuals, 
constituting only a small fraction of the total species population. Additional data collection may lead to identification of other crucial sites for these species across their range.

Our results provide insights for evaluating migration network robustness, which is useful for guiding the rational allocation of conservation efforts and funds. To effectively conserve migratory species, we suggest that policy makers should emphasize the designation and management of crucial sites that strongly contribute to the migration network's connectivity and exhibit a high degree of habitat loss. Among the crucial sites identified in this study, $42 \%$ are not currently protected (Appendix S5.9). These sites should be prioritized for listing under the flyway site networks, for example, of the East Asian-Australasian Flyway Partnership.

Our presently described analytical framework involves a network approach, and can be applied to help predict and prevent migration network collapse, and to thus guide recommendations for regional policy makers. Our approach could be applied in addition to the present criteria used to identify important habitats that need protection. For example, the current Ramsar Sites Criteria define a wetland as internationally important if it exhibits high biodiversity, if vulnerable species are observed, or if it is a special wetland type ${ }^{212}$. However, these criteria do not account for the wetland's importance in terms of the migration network's connectivity.

\subsection{Acknowledgements}

We thank N. Batbayar, F. Xu, W. Zhang, J. Wei, B. Wielstra, G. Liu, H. Luo, S. Balachandran, S. K. Behera, M. Ponnaian, Z. Lv, W. Wu, B. Gao, T. Zhang, and all the people who assisted the Department of Earth System Science of Tsinghua University (DESS), US Geological Survey (USGS), Food and Agriculture Organization of the United Nations (FAO) in collecting and organizing the tracking data. We thank Y. Wang for her suggestions for data analysis. Financial support was provided by the National Key R\&D Program of China (No. 2017YFA0604404), the National Natural Science Foundation of China (No. 41471347) and Chinese Scholarship Council (No.201600090128). 


\section{Supplementary information}

The supplementary information includes a summary of the utilized tracking data (Appendix S5.1), capture sites (Appendix S5.2), migration lags of study species (Appendices S5.4 \& S5.5), overall network metrics of migration networks (Appendices S5.6-S5.8), information regarding the identified crucial sites (Appendix S5.9), plots for the sensitivity analysis of the effect of number of tracks on site detection (Appendix S5.3), and species-specific comparison between network metrics (Appendix S5.10).

Appendix S5.1 A summary of tracking data, with species, scientific name, number of spring and autumn tracks and number of tracked birds. Sources of the tracking data were described with study name in movebank.org.

\begin{tabular}{|c|c|c|c|c|c|c|}
\hline $\begin{array}{l}\text { Common } \\
\text { name }\end{array}$ & $\begin{array}{l}\text { Scientific } \\
\text { name }\end{array}$ & Flyway & $\begin{array}{l}\text { Northward } \\
\text { tracks }\end{array}$ & $\begin{array}{l}\text { Southward } \\
\text { tracks }\end{array}$ & $\begin{array}{l}\text { Total } \\
\text { individuals }\end{array}$ & Data source \\
\hline $\begin{array}{l}\text { Swan } \\
\text { Goose }\end{array}$ & $\begin{array}{l}\text { A. } \\
\text { cygnoides }\end{array}$ & EAAF & 7 & 42 & 81 & $\begin{array}{l}2015 \text { Tsinghua } \\
\text { Waterfowl } \\
\text { FAO-USGS_Mongolia- } \\
\text { East FAO-USGS } \\
\text { Mongolia-2014 }\end{array}$ \\
\hline $\begin{array}{l}\text { Greater } \\
\text { White- } \\
\text { fronted } \\
\text { Goose }\end{array}$ & $\begin{array}{l}\text { A. } \\
\text { albifrons }\end{array}$ & EAAF & 17 & 14 & 54 & $\begin{array}{l}2015 \text { Tsinghua } \\
\text { Waterfowl }\end{array}$ \\
\hline $\begin{array}{l}\text { Whooper } \\
\text { Swan }\end{array}$ & C. cygnus & EAAF & 3 & 7 & 10 & $\begin{array}{l}\text { FAO-USGS_Mongolia- } \\
\text { East }\end{array}$ \\
\hline $\begin{array}{l}\text { Bar- } \\
\text { headed } \\
\text { Goose }\end{array}$ & A. indicus & $\mathrm{CA}$ & 36 & 45 & 93 & $\begin{array}{l}\text { FAO-USGS_China- } \\
\text { Qinghai } \\
\text { FAO-USGS_India-2009 } \\
\text { USGS_India-Nepal } \\
\text { FAO-USGS_Mongolia- } \\
\text { West }\end{array}$ \\
\hline
\end{tabular}


Appendix S5.2 Information of capture sites for the tracked individuals.

\begin{tabular}{|c|c|c|c|c|c|c|c|}
\hline Source study & Species & Country & Site & $\begin{array}{l}\text { Latitud } \\
\text { e }\end{array}$ & $\begin{array}{l}\text { Longitud } \\
\mathrm{e}\end{array}$ & Seasonal & $\begin{array}{l}\text { Data } \\
\text { Period }\end{array}$ \\
\hline $\begin{array}{l}2015 \text { Tsinghua } \\
\text { Waterfowl }\end{array}$ & A. albifrons; & China & Poyang & $29.1^{\circ} \mathrm{N}$ & $116.3^{\circ} \mathrm{E}$ & Non- & 2015- \\
\hline $\begin{array}{l}2015 \text { Tsinghua } \\
\text { Waterfowl }\end{array}$ & A. cygnoides & China & $\begin{array}{l}\text { Hulun } \\
\text { Lake }\end{array}$ & $48.3^{\circ} \mathrm{N}$ & $117.4^{\circ} \mathrm{E}$ & Moulting & $\begin{array}{l}2016- \\
2018\end{array}$ \\
\hline $\begin{array}{l}\text { FAO-USGS } \\
\text { Mongolia-East }\end{array}$ & $\begin{array}{l}\text { A. } \\
\text { cygnoides; } \\
\text { C. cygnus }\end{array}$ & Mongolia & $\begin{array}{l}\text { Mongol } \\
\text { Daguur }\end{array}$ & $49.7^{\circ} \mathrm{N}$ & $114.7^{\circ} \mathrm{E}$ & Moulting & $\begin{array}{l}2006- \\
2009\end{array}$ \\
\hline $\begin{array}{l}\text { FAO-USGS } \\
\text { Mongolia-2014 }\end{array}$ & A. cygnoides & Mongolia & $\begin{array}{l}\text { Mongol } \\
\text { Daguur }\end{array}$ & $49.6^{\circ} \mathrm{N}$ & $115.6^{\circ} \mathrm{E}$ & Moulting & $\begin{array}{l}2014- \\
2016\end{array}$ \\
\hline $\begin{array}{l}\text { FAO-USGS } \\
\text { China-Qinghai }\end{array}$ & A. indicus & China & $\begin{array}{l}\text { Qinghai } \\
\text { Lake }\end{array}$ & $37.1^{\circ} \mathrm{N}$ & $99.4^{\circ} \mathrm{E}$ & Breeding & $\begin{array}{l}2007- \\
2013\end{array}$ \\
\hline $\begin{array}{l}\text { FAO-USGS } \\
\text { India-2009 }\end{array}$ & A. indicus & India & $\begin{array}{l}\text { Chilika } \\
\text { Lake }\end{array}$ & $19.7^{\circ} \mathrm{N}$ & $85.3^{\circ} \mathrm{E}$ & $\begin{array}{l}\text { Non- } \\
\text { breeding }\end{array}$ & $\begin{array}{l}2008- \\
2010\end{array}$ \\
\hline $\begin{array}{l}\text { USGS } \\
\text { India-Nepal }\end{array}$ & A. indicus & Nepal & $\begin{array}{l}\text { Chitwan } \\
\text { National } \\
\text { Park }\end{array}$ & $27.7^{\circ} \mathrm{N}$ & $84.3^{\circ} \mathrm{E}$ & $\begin{array}{l}\text { Non- } \\
\text { breeding }\end{array}$ & 2005 \\
\hline $\begin{array}{l}\text { FAO-USGS } \\
\text { Mongolia-West }\end{array}$ & A. indicus & Mongolia & $\begin{array}{l}\text { Terkhiin } \\
\text { Tsagaan } \\
\text { Lake }\end{array}$ & $48.2^{\circ} \mathrm{N}$ & $99.7^{\circ} \mathrm{E}$ & Moulting & $\begin{array}{l}2008- \\
2010\end{array}$ \\
\hline
\end{tabular}

Appendix S5.3 Sensitivity analysis of the effect of the number of tracks on detection of sites for (a) southward migration of swan Geese; (b) southward migration of bar-headed geese.
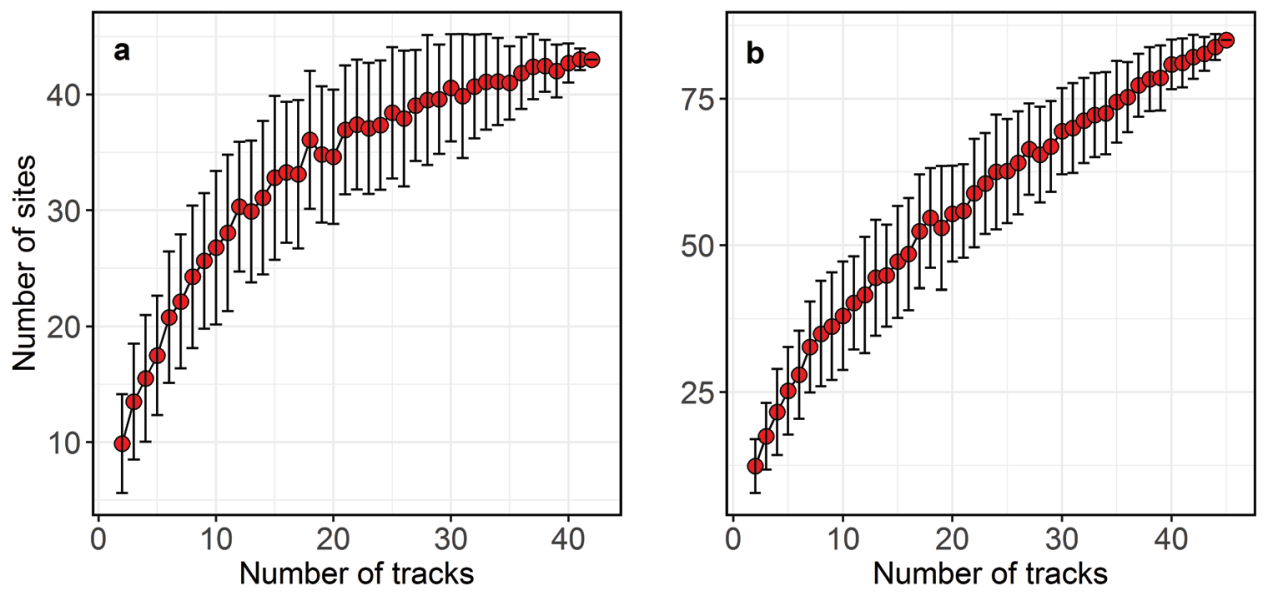
Appendix S5.4 Median, maximum, and 95\% confidence interval for median migration lag of autumn and spring migrations of Bar-headed Goose, Greater White-fronted Goose, Swan Goose, and Whooper Swan in kilometers.

\begin{tabular}{llllllr}
\hline Species & Direction & Median & Maximum & \multicolumn{2}{c}{$95 \%$ CI $(\mathrm{km})$} & Sample size \\
& & & & Upper & Lower & \\
\hline Swan Goose & Northward & 168 & 1396 & 335 & 118 & 36 \\
& Southward & 168 & 2063 & 227 & 125 & 215 \\
Greater White- & Northward & 217 & 3018 & 299 & 148 & 147 \\
fronted Goose & Southward & 1430 & 3180 & 1790 & 350 & 40 \\
Whooper Swan & Northward & 261 & 1152 & 445 & 145 & 16 \\
& Southward & 411 & 885 & 606 & 193 & 22 \\
Bar-headed & Northward & 194 & 2295 & 229 & 139 & 189 \\
Goose & Southward & 202 & 1369 & 231 & 185 & 118 \\
\hline
\end{tabular}

Appendix S5.5 Violin plots of migration lags (box plots with rotated kernel density plots on each side) in northward and southward migration for bar-headed geese (BHG), greater white-fronted geese (GWFG), swan geese (SG), and whooper swans

(WS). The 'migration lag' is the birds' travel distance from one site to the next.
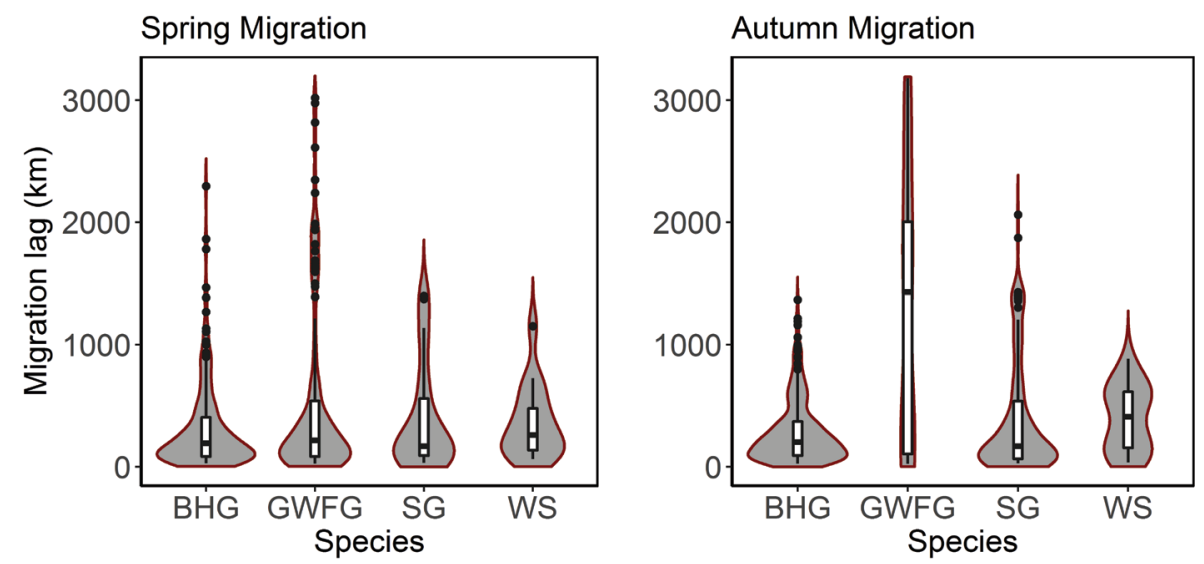


\section{Appendix S5.6 Overall network metrics}

Methods. We calculated several overall networks to indicate the connectivity and characteristics of the studied migration networks. These overall network metrics include the number of nodes, network size, graph density, graph diameter, modularity, number of alternative routes and shortest path length between the southernmost non-breeding and northernmost breeding sites (hereafter as path length).

The 'size of a network' is the total number of connections between nodes which indicates the total number of sites used by the birds ${ }^{213}$. 'size of a network' indicates the total number of possible steps the bird can take in the migration networks. The 'graph density' is the proportion of actual connections in all possible connections in a network ${ }^{213}$. The higher the 'graph density', the more intensively the sites are connected in the migration networks. The 'graph diameter' is minimum number of steps for the bird to travel between the two farthest sites (breeding and non-breeding sites) in the network. We measured weighted path lengths between each pair of nodes in these networks by the Dijkstra algorithm ${ }^{214}$. 'Path length' is calculated by the weighted path length between the two farthest sites in the network which measures the lowest costs the bird has to make to move between the southernmost non-breeding and northernmost breeding sites. . 'Modularity' measures the degree to which a network were divided into smaller modules. Modules were formed by a group of nodes in the network, maximizing the connectivity within each of the modules and minimizing the connectivity between different modules ${ }^{1}$. The higher the modularity, the denser the connections between the nodes within modules, but the sparser the ties connecting these different modules ${ }^{1}$. We measured the number of alternative routes between the southernmost non-breeding and northernmost breeding sites by analysing the adjacency matrix of sites 37,215.

We generated 999 random networks whose nodes were randomly generated within the extents of the species' movement as calculated from the available tracking data. The random networks had the same number of nodes, and the weighted connections between these nodes were calculated with the same methods as for the observed migration networks. We compared graph density, modularity, number of alternative routes and path length of the migration networks with 999 random networks which were used as null models (Appendix S5.8) to indicate how the empirical migration networks are different from the random configurations. 
Results. The diameters of most migration networks of the study species was 2 (Appendix S5.7), which means at least one stopover site is required for a successful travel between their northernmost breeding sites and southernmost non-breeding sites. However, for bar-headed geese in southward migration, at least three stopover sites are required for completing the full migration. bar-headed geese had northward and southward migration networks with a higher graph density and number of alternative routes, a lower path length and modularity than the random networks (Appendix S5.8). The southward migration network of greater white-fronted geese had a lower graph density and higher modularity compared to the random ones. Although their northward migration network had a higher graph density compared to the random networks, its path length was higher than the random ones. The southward migration network of swan geese had a higher graph density and a lower path length, but a lower number of alternative routes than the random networks. 


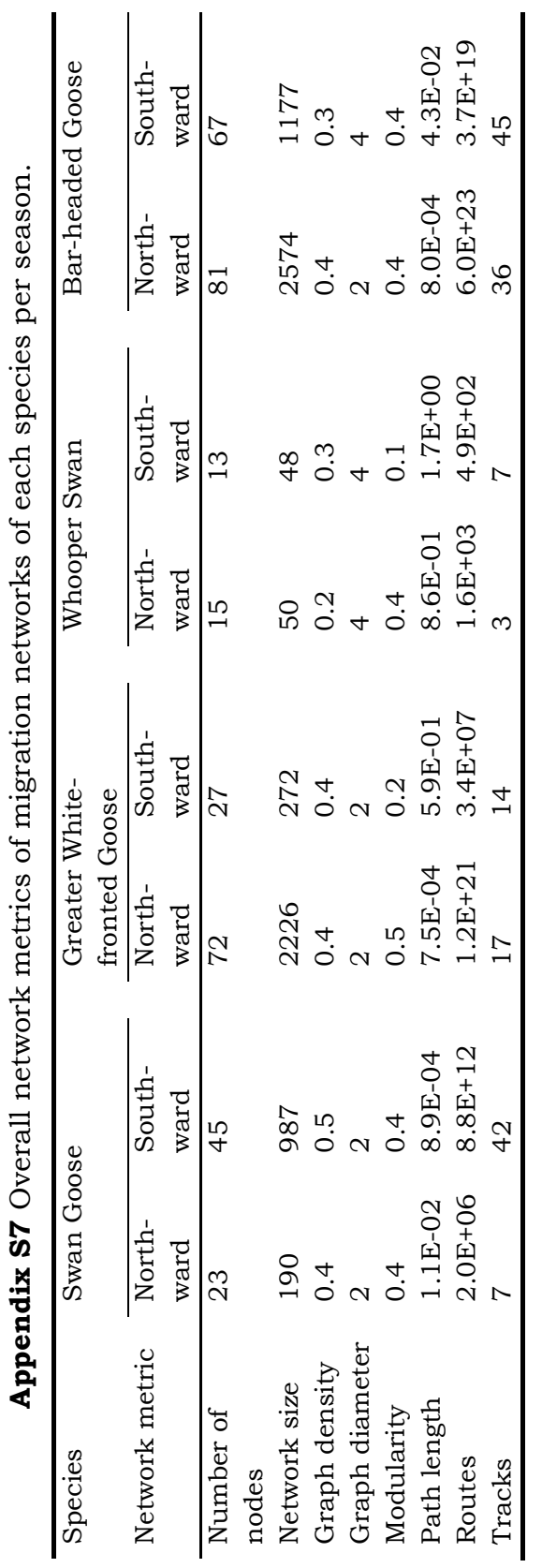


Appendix S5.8 Comparison of empirical migration networks with random networks. Red triangles are the value of the corresponding metrics in the empirical migration networks, and the grey circles and bars represent mean and standard deviation of these corresponding metrics of the 999 random networks. Species names are abbreviated as BHG (bar-headed goose), GWFG (greater white-fronted goose), SG (swan goose), and WS (whooper swan), followed by a letter "A" or "S"

which refers to southward and northward migration, respectively.
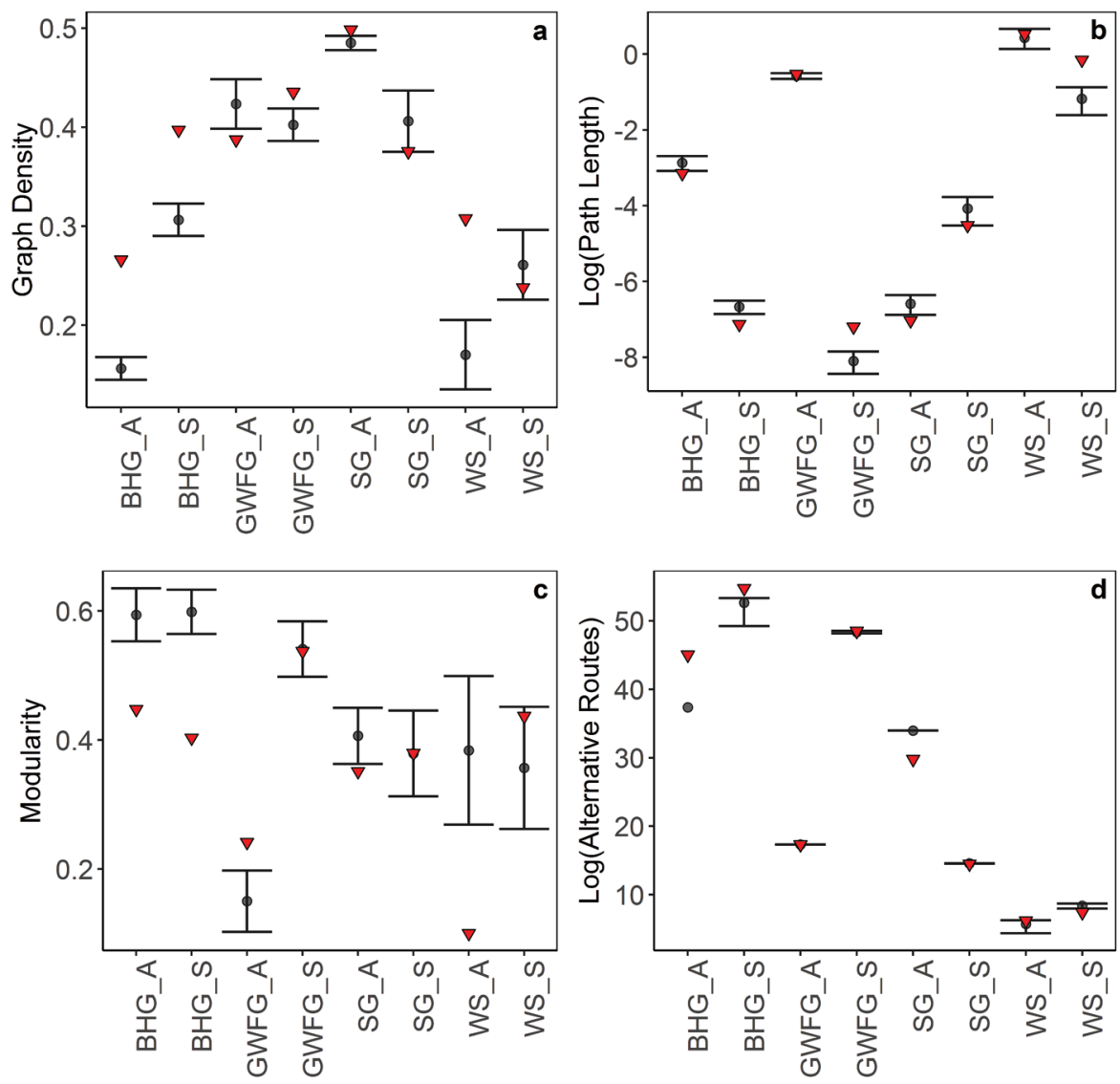
Appendix S5.9 Differences in the effect indices of betweenness, degree, and node resistance for each studied migration network. The letters on the boxes represent the identical groups as identified by the multiple comparison test at p=0.05. a, c, e are northward migration networks of swan geese, greater white-fronted geese, and bar-headed geese, respectively; b, d, f are southward migration networks of swan geese, greater white-fronted geese, and bar-headed geese, respectively.

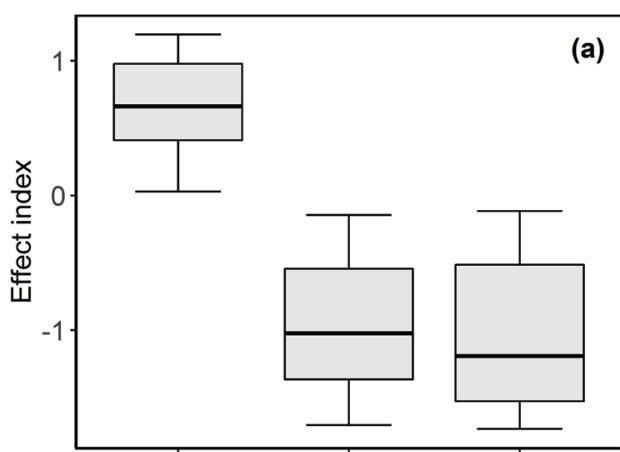

Betweenness Degree Node Resistance Network metrics

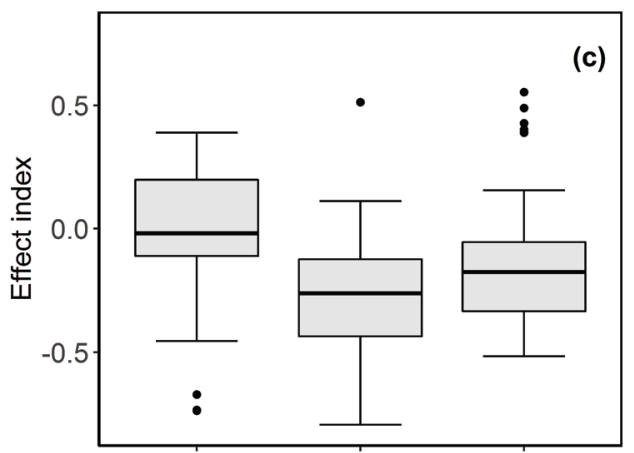

Betweenness Degree Node Resistance Network metrics

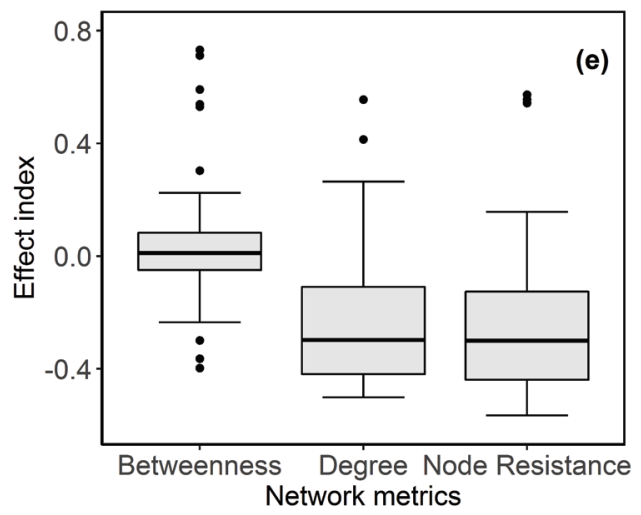

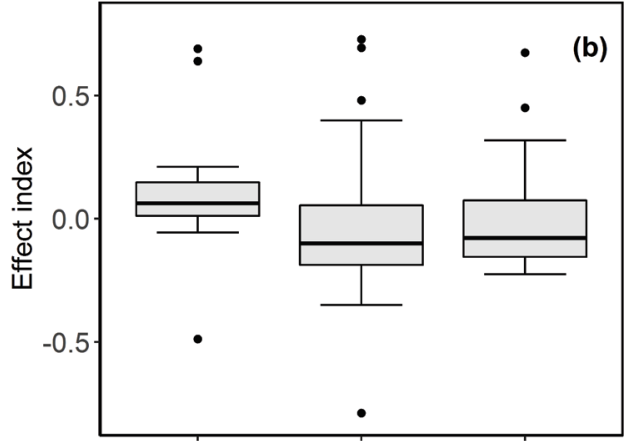

Betweenness Degree Node Resistance Network metrics

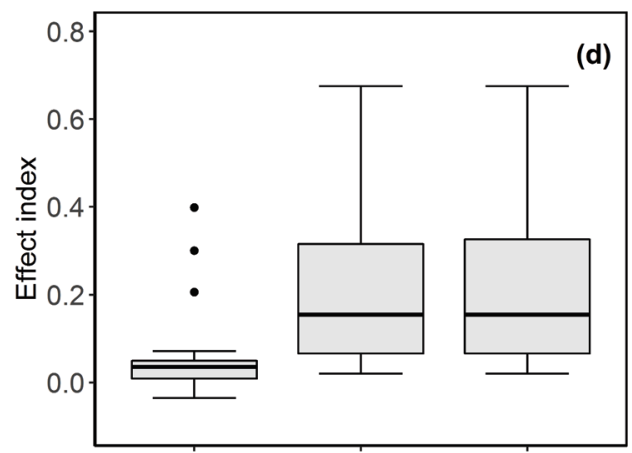

Betweenness Degree Node Resistance Network metrics

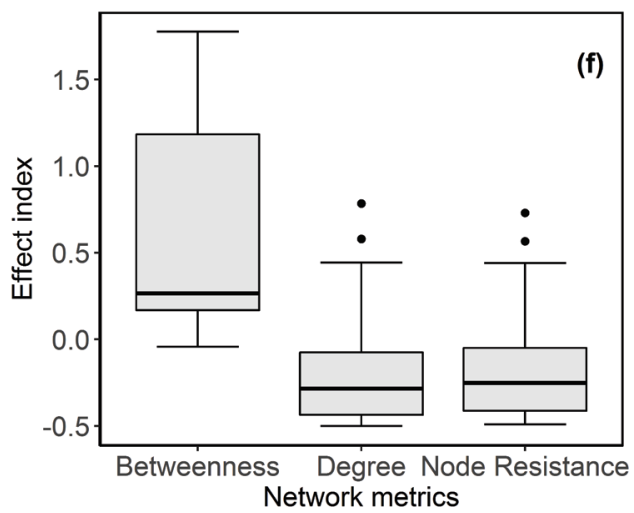


Appendix S5.10 Information of identified crucial sites. Species names are abbreviated as BHG (bar-headed goose), GWFG (greater white-fronted goose), SG (swan goose), and WS (whooper swan).

\begin{tabular}{|c|c|c|c|c|c|c|c|c|}
\hline ID & Name & Country & Latitude & Longitude & $\begin{array}{l}\text { Habitat } \\
\text { loss }\end{array}$ & Protection & Species & Direction \\
\hline 1 & Dafeng & China & 33.5093 & 120.6183 & $1.1 \%$ & Yes & SG & Northward \\
\hline 2 & Tongyu & China & 44.6616 & 122.5036 & $16.1 \%$ & Yes & SG & Northward \\
\hline 3 & Donghai & China & 34.4257 & 119.0113 & $40.0 \%$ & Yes & SG & Southward \\
\hline 4 & Dongying & China & 37.3762 & 118.9935 & $5.5 \%$ & Yes & SG & Southward \\
\hline 5 & Leting & China & 38.8283 & 118.4182 & $6.3 \%$ & No & SG & Southward \\
\hline 6 & Xingcheng & China & 40.3308 & 120.5196 & $14.8 \%$ & Yes & SG & Southward \\
\hline 7 & $\begin{array}{l}\text { Horqin Left } \\
\text { Back }\end{array}$ & China & 43.2624 & 122.8737 & $2.5 \%$ & Yes & SG & Southward \\
\hline 8 & Hexigten & China & 43.2912 & 116.5685 & $2.8 \%$ & Yes & SG & Southward \\
\hline 9 & $\begin{array}{l}\text { Horqin Left } \\
\text { Middle }\end{array}$ & China & 44.1948 & 121.2612 & $9.5 \%$ & Yes & SG & Southward \\
\hline 10 & $\begin{array}{l}\text { Xinbarag } \\
\text { Right }\end{array}$ & China & 48.5478 & 117.2547 & $1.5 \%$ & Yes & SG & Southward \\
\hline 11 & Zhongmou & China & 34.9192 & 113.9925 & $19.4 \%$ & Yes & GWFG & Northward \\
\hline 12 & Laixi & China & 36.9554 & 120.4506 & $10.0 \%$ & No & GWFG & Northward \\
\hline 13 & Xinmin & China & 42.2175 & 122.6565 & $0.3 \%$ & No & GWFG & Northward \\
\hline 14 & Kobyayskiy & Russia & 64.8114 & 125.6569 & $9.1 \%$ & No & GWFG & Northward \\
\hline 15 & Bulunskiy & Russia & 70.2565 & 125.9493 & $0.2 \%$ & No & GWFG & Northward \\
\hline 16 & $\begin{array}{l}\text { Horqin Right } \\
\text { Middle }\end{array}$ & China & 44.5601 & 121.9112 & $7.4 \%$ & No & GWFG & Southward \\
\hline 17 & Longjiang & China & 47.3683 & 123.4235 & $0.6 \%$ & No & GWFG & Southward \\
\hline 18 & Arun & China & 48.4606 & 123.1705 & $0.2 \%$ & No & GWFG & Southward \\
\hline 19 & Chengalpattu & India & 12.4630 & 80.1382 & $47.7 \%$ & No & BHG & Northward \\
\hline 20 & Khordha & India & 19.8819 & 85.2713 & $6.8 \%$ & Yes & BHG & Northward \\
\hline 21 & Bemetara & India & 21.5390 & 81.5831 & $3.0 \%$ & No & BHG & Northward \\
\hline 22 & Chandmani & Mongolia & 45.2256 & 98.2113 & $4.9 \%$ & No & BHG & Northward \\
\hline 23 & Giridih & India & 24.4330 & 86.2508 & $3.6 \%$ & Yes & BHG & Southward \\
\hline 24 & Baatsagaan & Mongolia & 45.7136 & 99.3265 & $4.3 \%$ & Yes & BHG & Southward \\
\hline
\end{tabular}


A network approach to prioritize conservation efforts for migratory birds | 113 



\section{Range shifts of migratory hosts facilitate avian influenza panzootics via crossroads of flyways}

Xu Y., van der Jeud H., Si Y., Herbert H. H. T., Fouchier R. A. M., Guillemain M., Caizergues A., Li Z., Eikelboom J. A. J., Xu C., Thorup K., von Rönn J., Gong P,. de Boer W. F. Range shifts of migratory hosts facilitate avian influenza panzootics via crossroads of flyways. 


\section{Abstract}

Panzootics of infectious diseases, like avian influenza, have caused huge impacts on human and animal health, as well as the economy. Avian influenza viruses are carried around the world through the crossroads of flyways of migratory birds. Identification of these crossroads, i.e., locations where different populations mix and viruses change hosts, are crucial for surveillance and early warning of long-distance virus transmission.

However, little is known about the distribution of these crossroads, and how global changes shift these crossroad locations and influence the intensity of crossing. Using a module detection algorithm and bridging centrality measurements from network theory, we mapped intra- and inter-species crossroads for populations of 47 migratory waterfowl species in Eurasia and North America, as well as changes of these crossroads from 1950 to 2019. Intra-species crossroads mainly occur in the northernmost and southernmost areas in North America, and in the eastern areas of Eurasia. Several highly susceptible species, i.e., mallard, Eurasian teal, and Canada goose, bridge the migration routes of other species more than less susceptible species, indicated by a higher inter-species crossing intensity. Over the last seven decades, North American species cross more within species, while Eurasian species cross more across species. A clear pattern of a northward shift in North American species and eastward shift in Eurasian species is associated with these increases in crossing intensity. A range shift of migratory hosts driven for example by climate warming and land use change may thus result in an increasing risk of long-distance transmissions and broad-scale outbreaks of infectious diseases by increasing the crossing intensity within and between species. 


\subsection{Introduction}

The outbreak and spread of avian influenza viruses continue to cause huge economic loss and serious health problems for humans, wildlife, and poultry. Wild birds, particularly those of the order Anseriformes, are the natural reservoir for low pathogenic avian influenza viruses (LPAIV) ${ }^{247}$. When introduced to poultry, LPAIV subtype H5 and H7 can evolve to highly pathogenic avian influenza virus (HPAIV), such as H5N1 and H5N8, and cause substantial diseases in poultry, wild birds, and humans 216-218. Although poultry industry are enormously impacted by HPAIV and poultry trade may play a role in HPAIV transmission ${ }^{219}$, the seasonal migration of wild birds is thought to play a key role in the rapid transmission of HPAIV within and across continents, facilitating panzootics of avian influenza viruses $59,60,220$.

Both HPAIV H5N8 and H5N1 were originally detected in poultry in East Asia and then spread westwards and eastwards. For instance, HPAIV H5N8 rapidly spread from East Asia to Europe and North America in 2014. Wild birds in East Asia probably carried the virus to breeding grounds in Russia that are shared by migratory birds wintering in Europe or North-America, and then these birds carried the virus across the world during their autumn migration, which subsequently resulted in the global spread of HPAIV H5N8 60,221 . Most migratory birds travel seasonally within specific migration routes, and the crossroads of these routes from which birds travel to nonbreeding areas in different regions, e.g., Middle East, Europe, Africa, and North America, can facilitate a global spread of HPAIV. To better understand the global transmission of HPAIV, intensive surveillance and early detection of the virus in migratory birds at the crossroads of migration routes can therefore be most cost-effective and efficient. However, our understanding on the occurrence of these crossroads and connectivity between distant nonbreeding grounds is limited 60 .

The administrative flyways, i.e., the nine major waterbird flyways of the world (wpe.wetlands.org/Iwhatfly), are generally used for studying the role of migratory birds in global transmission of HPAIV 59,222,223. However, these flyways are too broadly defined and geographically indefinite to circumscribe the crossroads of migratory flyways of migratory bird species from isolated wintering grounds, especially for different populations of the same species. Although satellite tracking data can provide detailed information about the migratory movement of individuals, it is challenging to identify the precise 
location of the crossroads of migration routes due to a limited number of tracked birds and populations.

Thousands of birds of various species are being ringed each year, and movement data of these birds together with the adequate approaches give opportunity for a better understanding of crossroads. With bird banding recovery data, Buhnerkempe, et al. ${ }^{46}$ proposed a novel approach, using a module detection technique from network theory for the identification of migration routes of different populations from the same species. According to the definition of module structures in a network ${ }^{1}$, the nodes within a module are densely connected, while nodes from different modules are not, or sparsely connected. Therefore, a module in the migration network of a bird species can be defined as the migration route of a population of that species. The migration route is of course the whole aerial network but the locations where birds land to rest or forage are named "sites" by ecologists but "nodes" by network theoreticians. Bridging centrality is a network metric measuring the importance of a node connecting different modules in a network ${ }^{224}$. The nodes with a high bridging centrality value in a bird migration network are key sites connecting different populations using different migration routes and, therefore, can be defined as intra-species crossroads where different populations have a high chance of contact (Figure 6.1a). Besides, two different populations from one species can also be bridged by another particular species (that may just act as a carrier species and may or may not be infected by the pathogen), i.e., an interspecies crossing (Figure 6.1b).

The changing environment can result in shifts in patterns of spatial distribution and movements of animals, especially for migratory bird species with their high mobility and sensitivity to, for example, changing temperatures 52 . There are several studies showing that the distribution of migratory birds in North America has shifted northward or their migration distance has been shortened due to climate warming, but also due to land use changes like increasing food availability from agricultural expansion 53,225. Besides a northward shift in North American species, Eurasian bird species generally shifted north-eastwards according to modelling 54,226 and field studies 227,228 229-231, due to climate and land use changes. These range shifts alter species distribution and species richness patterns, and subsequently affect the geographical location and the intensity of crossing at the crossroads where populations and species mix. 
In this study, we test whether a northward and/or eastward range shift will increase crossing intensity within and between migratory species. With a module detection approach and bridging centrality measurement, we identify the crossroads along migratory routes of 47 migratory waterfowl species in the northern hemisphere. We identify the intra-species crossroads and the inter-species crossing intensity, i.e., the degree to which one species bridge migration routes of other species. By measuring the changes in migration routes and the intensity and locations of potential crossroads from 1950-1984 to 1985-2019, we test whether the range shifts of migratory birds under the influence of global environmental changes facilitate virus transmission in forms of increasing crossing intensity within and between species. The results provide insights for prioritizing sites for targeted disease surveillance and control in the framework of the global spread of novel HPAIV strains.
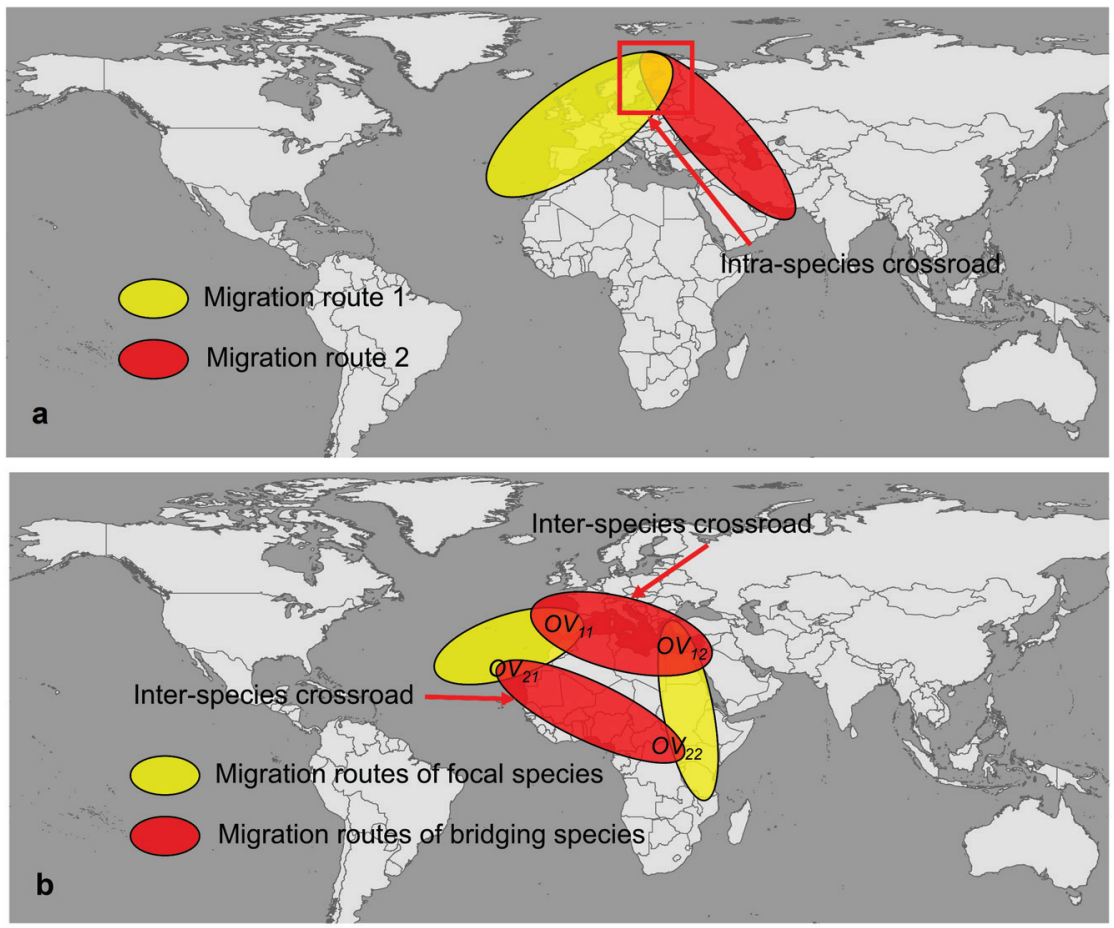

Figure 6.1 An illustration of the definition for (a) intra-species crossroad and (b) inter-species crossroad. For (a), migration routes of different populations of one species are defined by the classified modules; the intra-species crossroad is defined by sites with a high local bridging centrality value. For (b), the details of calculations for inter-species crossing intensity were described in the main text. 


\subsection{Methods}

\section{Data}

To construct the migration networks of migratory species of the order Anseriformes, we obtained a total of 5,473,545 bird marking (i.e., ringing, banding, nasal saddles) and recovery/re-sighting records from Bird Banding Laboratory of the United States Geological Survey Patuxent Wildlife Research Center, Laurel, United States (3,812,418 records), EURING databank, European Union for Bird Ringing (1,661,127 records), and the Office Français de la Biodiversité, France (29,530 records). We excluded those species with less than 1000 records. Consequently, a total of 26 species in North America and 21 species in Eurasia were included in subsequent analyses (Appendix S6.1). The data include bird ID, date, latitude and longitude of each individual marking and resighting record, with latitude and longitude. To analyse broad temporal changes and to include sufficient samples in each of the time periods, we separated the bird data reported from 1950-1984, from those reported from 1985-2019, merging 35 years' data in each time period.

\section{Network construction}

We constructed migration networks for each study species in the period 1950-1984 and the period 1985-2019. Each $200 \times 200 \mathrm{~km}$ grid cell over the geographic ranges of each species was considered a node ${ }^{46}$. The marking and recovery/re-sighting records within each grid cell were assigned to the corresponding node as attributes. To reduce the spatial sampling bias caused by a small sample size in some of the regions (Appendix S6.1), we only included nodes with $\geq 3$ re-sighting/recovery records for the focal species in the subsequent network analysis. If one individual bird was marked in one of the nodes and re-sighted or recovered in another node, a connection was assigned between the two nodes. The weight of the connections represents conditional probability of movement conditional on the efforts for recovery/re-sighting. The conditional probability was defined by the count of the marking and recovery/re-sighting records between corresponding nodes. We performed the network construction with package 'igraph' 232 in R 3.5.0 233 .

\section{Migration routes and crossroads identification}

The migration routes of different populations for a given species and period were identified by the modules in the constructed networks with a Walktrap community detection algorithm ${ }^{234}$. The nodes were assigned to different 
modules to maximize the connectivity within each of the modules, and to minimize the connectivity between different modules ${ }^{1}$. As a result, the Walktrap algorithm assigned a membership ID to each node in the migration networks, defining which migration route the node belongs to.

Intra-species crossroads. The intensity of intra-species crossing was quantified by the bridging centrality value. For each of the nodes in a bird migration network, we calculated a local bridging centrality ${ }^{17}$, which measures the degree to which a site connects different modules. The bridging centrality ranges from 0 to infinity, and the higher the value, the more important a site is for bridging different migration routes. For further calculation, the bridging centrality values for each species were standardized to range between 0 and 1 .

Inter-species crossroads. We defined the species bridging separate migration routes of another species (focal species; species $\mathrm{j}$ in Figure 6.1b) as a bridging species (species $\mathrm{i}$ in Figure 6.1b). The intensity at which a bridging species crossed the migration routes of the focal species, hereafter referred to as inter-species crossing intensity, was quantified by the intersection over groups ${ }^{235}$. Specifically, it was calculated from the size of the overlapping area between one migration route of the bridging species and one migration route of the focal species multiplied by the size of the overlapping area between that migration route of the bridging species and another migration route of the focal species. We calculated the inter-species crossing intensity for each species pair combination, including all migration routes of the two species, recording the maximum value as the final interspecies crossing intensity for this pair. The final inter-species crossing intensity value for each pair of bridging species $(i)$ and focal species $(j)$ is:

$$
I O G_{i j}=\max \left(\mathrm{OV}_{11} \times \mathrm{OV}_{12}, \mathrm{OV}_{21} \times \mathrm{OV}_{22}\right)
$$

Taking the migration routes of the bridging and focal species in Figure $1 \mathrm{~b}$ as an example, and assuming that species $i$ and species $j$ both have two migration routes, the number of overlapping grid cells between migration route 1 of species $i$ and migration route 2 of species $j$ can be calculated from the overlapping sections in Figure $1 \mathrm{~b}$, i.e., $O V_{12}$.

\section{Analysis}

To measure species range shifts, we quantified the northernmost and southernmost boundary of all distinguished migration routes for a given species and period by the upper and lower limits of $95 \%$ confidence interval 
(95\% CI) of the latitudes of marking and recovery/re-sighting records, respectively. We quantified their easternmost and westernmost boundary by upper and lower limits of 95\% CI of the longitudes. We then quantified their northward and/or eastward range shifts by the difference in these boundaries between the two periods. A positive change in the latitudes represents a northwards shift and a positive change in the longitudes represents an eastward shift for the species. Additionally, we calculated the changes in species richness per grid cell in the study area from 1950-1984 to $1985-2019$.

We mapped, under the World Geodetic System 1984, the intra-species crossroads by averaging the standardized bridging centrality values for different species per grid cell per period, and quantified the changes by the differences in the average bridging centrality between 1950-1984 and 19852019. A positive value for this change represents an increase in the intensity of intra-species crossing in that grid cell, while a negative value represents a decrease. Similarly, to quantify the intensity of inter-species crossing and the changes thereof, for each combination of bridging and focal species, we measured the difference in their inter-species crossing intensity between the two periods.

To summarize the changes in the crossing intensity in North America and Eurasia, for each grid cell, we summed the standardized bridging centrality values for all species per 35-year period. We tested whether there was a significant difference in the summed bridging centrality between the two periods using a Mann-Whitney U rank sum test, as it did not follow a normal distribution (Eurasia: Kolmogorov-Smirnov normality test, $N=1015$, $D=0.127, P<0.001$; North America: Kolmogorov-Smirnov normality test, $N$ $=1580, D=0.236, P<0.001)$.

\subsection{Results}

For North American species, there was a clear pattern of a northward shift in the studied migratory species (Figure 6.2). Consequently, the species richness in Mexico and South America decreased while that in Northwest Canada, Alaska and mainland of United States increased (Figure 6.2a). Both their southernmost (median latitude change $=0.32^{\circ} / 26 \mathrm{~km}$ at $40^{\circ} \mathrm{N}$ ) and northernmost (median latitude change $=0.21^{\circ} / 17 \mathrm{~km}$ at $40^{\circ} \mathrm{N}$ ) spatial boundary of distributions moved northwards from 1950-1984 to 1985-2019 (Figure 6.2b). For Eurasian species, we observed an eastward shift. Species 
richness in Portugal, Spain, France, and Sweden decreased while it increased in a large part of Western Russia (Figure 6.2a). Both their westernmost (median latitude change $=0.96^{\circ} / 79 \mathrm{~km}$ at $40^{\circ} \mathrm{N}$ ) and easternmost $\left(\right.$ median latitude change $=1.21^{\circ} / 92 \mathrm{~km}$ at $40^{\circ} \mathrm{N}$ ) spatial boundary of distributions moved eastwards (Figure 6.2c).

Migration routes for North American waterfowl are mostly aligned in a north-south direction (Appendix S6.3), while Eurasian migration routes are more aligned in an east-west direction (Appendix S6.4). The intra-species crossroads mainly occur in the northernmost and southernmost areas in North American migration routes, but in the eastern areas of Eurasian migration routes (Figure 6.3a).

a
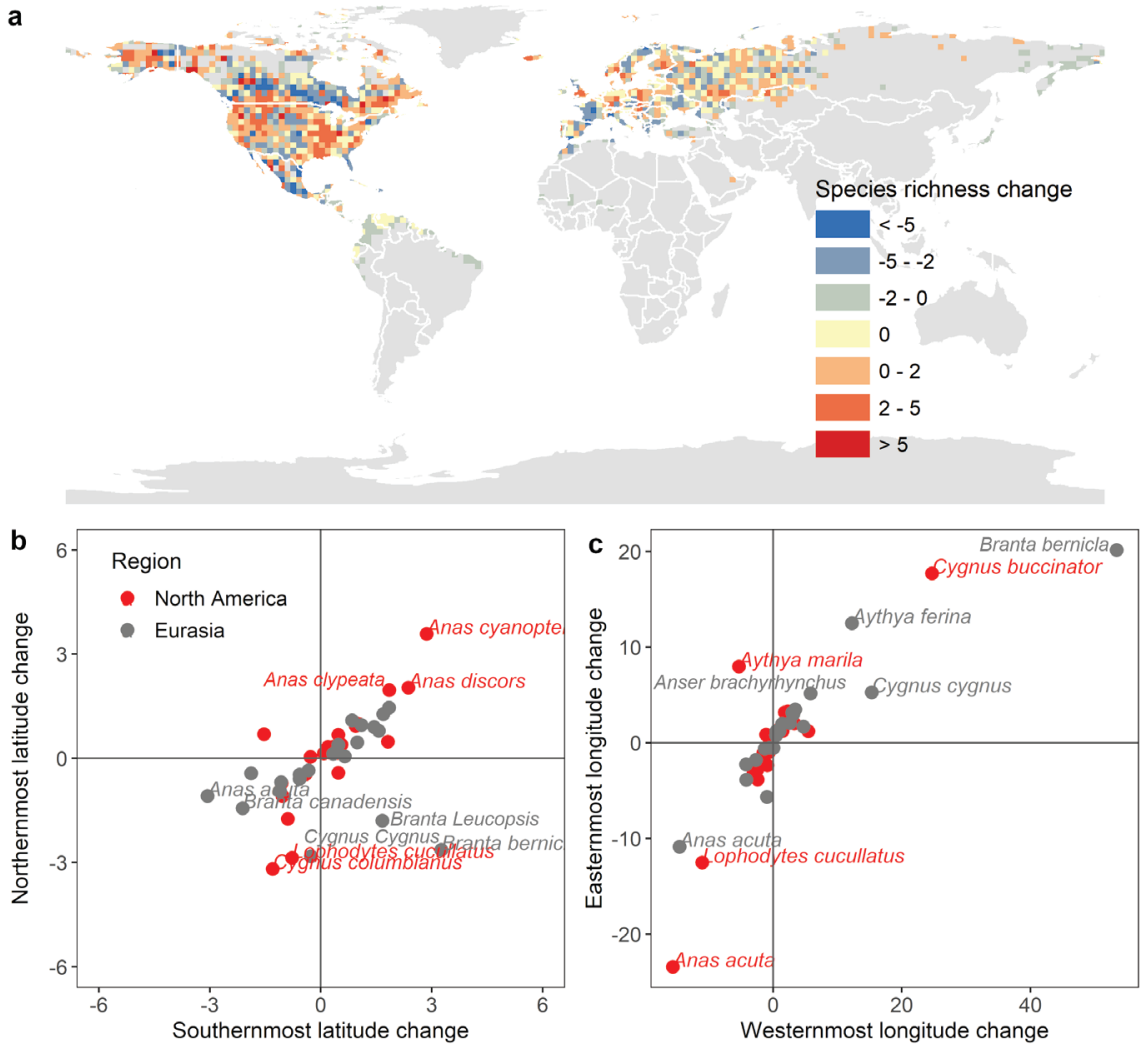

Figure 6.2 The changes in (a) the number of waterfowl species observed in each grid cell, (b) species southernmost and northernmost ranges for north American species, and (c) species westernmost and easternmost ranges for Eurasian species from 1950 to 2019 . The unit for (b) and (c) is degree $\left({ }^{\circ}\right)$. 
In most areas of North America, especially in the United States, the bridging centrality increased significantly from 1950-1984 to 1985-2019 (Figure 3b; Mann-Whitney U test, $N=1580, W=266980, P=0.04$ ). In contrast, the bridging centrality significantly decreased in Eurasia (MannWhitney U test, $N=1580, W=143530, P=0.002)$.

Compared to other regions, migration routes of different species spatially overlapped more in the United States, southern Canada, and Western Europe (Appendix S6.5). In the Eurasian migration routes, waterfowl species were most intensively bridged by Eurasian teal and mallard (summed IOG $_{i j}=$ 167188 and 146205; Figure 6.4b). In North America, mallard and Canada goose were the most important bridging species (14450461 and 2194326; Figure 6.4e).
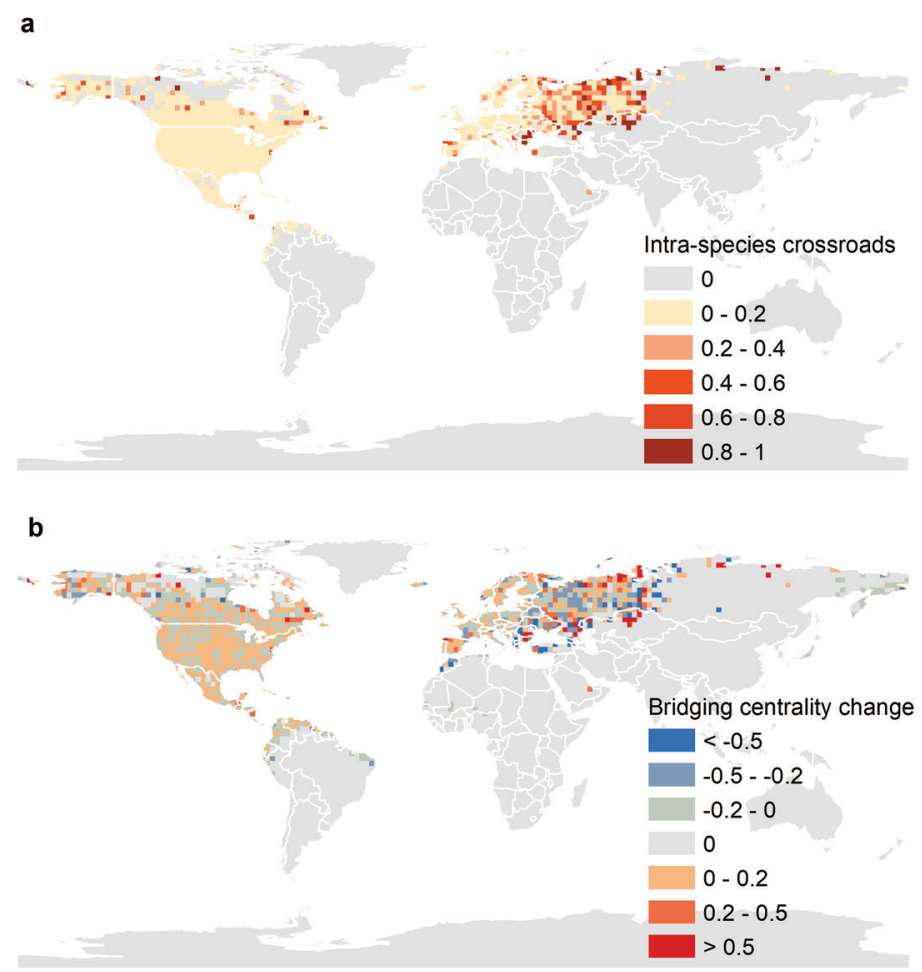

Figure 6.3 Intra-species crossroads. (a) the standardized intensity of intra-species crossing measured by the average bridging centrality in each of the grid cell in 1985-2019; (b) The corresponding changes of this intensity value in each grid cell between 1950-1984 and 1985-2019. 
In general, Eurasian species were becoming more intensively crossed by inter-species crossroads (an increase in inter-species crossing intensity in $71 \%$ of the species combinations; Figure $6.4 \mathrm{c}$ ), while no such pattern was observed in North American species (53\%; Figure 6.4f).
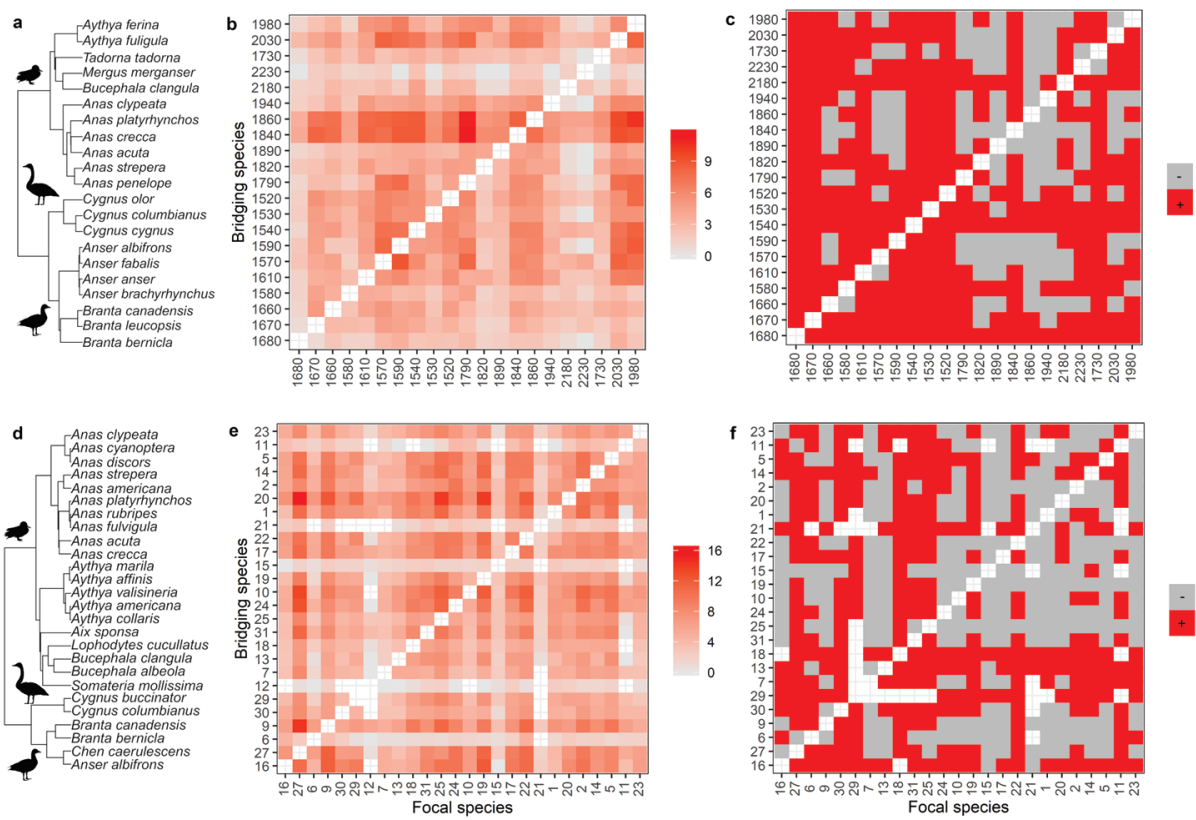

Figure 6.4 The intensity of one species bridging the migration routes of another species measured by inter-species crossing intensity. The subplots show (a) the phylogenetic tree, (b) log-transformed inter-species crossing intensity value, and (c) the change in inter-species crossing intensity from 1950-1984 to 1985-2019 for Eurasian species, and those (d)(e)(f) for North American species. A bridging species bridges the migration route of a focal species, and a focal species is the one connected by a bridging species (Figure 1). The numbers in the axes are species IDs corresponding to their scientific names in the phylogenetic tree and in Appendix $\mathrm{S} 1$. For (c) and (f), "+" and "-" represent an increase and a decrease in the interspecies crossing intensity, respectively. 


\subsection{Discussion}

We found that migratory birds in the northern hemisphere have shifted their distribution ranges from 1950-1984 to 1985-2019, but in different directions, as North American species shifted northwards, while Eurasian species mostly shifted eastwards (Figure 6.2). Along with these range shift patterns, species in both regions showed an increase in crossing intensity either in intra-species crossroads in North America, or in inter-species crossroads in Eurasia. Due to these changes in spatial patterns of bird migration, species displayed a higher crossing intensity, potentially resulting in a higher probability of disease transmission over long distance ${ }^{60}$.

The increasing crossing intensity with a range shift of migratory species can be explained by multiple factors. Migratory birds can shorten their migration in response to the global warming 236 by staying closer from their (northern) breeding ground during the non-breeding. This northward shifting of the southern boundaries of the non-breeding range of a species is called 'short-stopping', which is first observed in Canada geese in North America 225,228,229. A shorter migration distance can result in a smaller distribution range, and consequently in a higher probability of encounter (or contact) between individuals of different populations or species. Specifically, these range contractions lead to birds gradually avoiding regions where they little overlapped with each other in the past, and more concentrating in the same regions, hence the increased chances of mixing ${ }^{52}$, which may result in a higher mixing probability of these different populations at northern sites. This is consistent with what we observed in waterfowl species, i.e., an increasing probability of encounter both within and between species over the last decades in the United States and western Russia (Figure 6.3b; Figure 6.2a). However, it is also possible that a contraction of bird flyways, to some extent, could reduce the overall area where viruses like HPAIV could spread, for example keeping European areas further to the southwest out of risk zones.

The potential facilitating effect of the increase in encounter within and between species on disease transmission is probably coupled with other changes in host assemblages under global environmental changes. Firstly, most migratory waterfowl populations (especially geese species) have increased in North America and Europe making probability of contact (within and between species) higher. Although North American nonwaterfowl species generally experienced a population decline, population 
sizes of numerous waterfowl species in North America have increased by $>$ 50\% from 1970s ${ }^{49}$. Similarly, in Europe, some waterfowl species, e.g., geese species, have followed exponential population growth over the past decades 185. Increasing number of birds in a shrinking distribution range (Figure 6.2) contribute to a higher crossing intensity, but we are not certain how to factor into our analyses. Secondly, with a range shift, different populations of the same species may mix more intensively in regions where wetlands are relatively scarce. For example, we observed that different populations of the same species mix more from 1950-1984 to 1985-2019 in United States (Figure 6.3b) where the percentage of wetland cover is lower than the other regions in North America (Appendix S6.6). Thirdly, many waterfowl species in Europe and North America, especially geese, have managed to exploit agriculture resources and therefore shifted their local distributions, increasing their proximity to poultry ${ }^{119}$. Locations where wild bird species and poultry mix are favourable for local persistence and mutation of avian influenza viruses 218,237-239, further facilitating virus transmission among different host species. These changes can increase the (absolute and relative) density of competent hosts for HPAIV at crossroads, and subsequently facilitate virus transmission and virus jumping via increased contact rates between individuals and species 240,241 .

The range shifts of migratory host species may thereby facilitate panzootic of infectious diseases via a greater overall bird density and hence a higher probability of contact at crossroads, and are probably associated with climate and land use changes. Our results indicate that North American waterfowl are shifting northward while Eurasian waterfowl are shifting eastward, which is often attributed to climate warming 53,227. North American temperature isoclines are east-west oriented (except in Rocky Mountains and Alaska) 242 while the Eurasian ones are south-north oriented (https://www.pinterest.com/pin/312015080430448253/), thus, the north American isolines are mostly moving northward while the Eurasian ones are moving eastward under climate warming 243 . Therefore, North American and Eurasian birds shifted in different directions but similar patterns: shifting towards the continental "cold poles", i.e., northern Canada and Siberian Russia.

Besides climate change, human activities, e.g., increasing hunting pressures, agriculture and urban expansions, and regional conservation policies, may also be associated with these shifts (Teitelbaum et al. 2016). A 
larger cover of agricultural fields, can open up new suitable habitats for migratory birds 52,225. However, rapid habitat degradation and increased hunting pressure at non-breeding grounds, e.g., Yangtze River Basin and South America, coupled with climate warming, can force migrants to skip their southernmost habitats 116,188 . The Eurasian pattern of a eastward shift in waterfowl is accentuated by a land-use change patterns after the dissolution of the former USSR: the northern staging grounds of European Russia became less attractive due to grasslands getting overgrown with forest due to economically driven land abandonment 68,123. Eurasian Teal (Anas crecca) may, however, come back to a greater extent to southern Europe wintering grounds if experiencing favourable foraging conditions in the previous years ${ }^{244}$.

Different migration routes of the same species mainly cross in the northernmost and southernmost parts of their distribution areas in North America, and in the eastern areas in Eurasia (Figure 6.3a). Moreover, these northernmost areas in North America and the northeastern areas in Eurasia are important breeding grounds for many waterfowl species ${ }^{95}$. Naive juveniles have a higher infection rate than adult birds ${ }^{245}$, so the possibility that the avian influenza viruses infect another population is larger in these breeding areas $221,246,247$. In addition, disease surveillance is scarce in these Russian and Northern Canadian areas (Figure 6.3a) due to e.g., a low human density, and poor accessibility. Yet these areas are critical for early warning for the long-distance transmission of HPAIV. Similarly for Taymir migratory birds (though not included in this analysis), they breed in the same area, but can either migrate to Europe, or Kazakhstan, or China, connecting different routes which reach to very distant areas around the globe ${ }^{161}$.

For inter-species crossing, we found that mallard, Eurasian teal, and Canada goose, bridge the migration routes of other species more. Mallard is one of the species with the highest inter-species bridging intensity values both in North America and Eurasia (Figure 6.4bd), and numerous avian influenza viruses have been detected in mallards ${ }^{216}$, indicating they are highly susceptible to HPAIV infection. Canada goose, ranked second in the North American bridging species (Appendix S1), and has been reported in various HPAIV outbreak events in North America, so it is also a key player in the epidemiology of HPAIV 248,249. Moreover, our results also show a high inter-species bridging intensity value in dabbling ducks, e.g., Eurasian 
wigeon, mallard, and Eurasian teal (Figure 6.4bd). Dabbling ducks are the most infected group, with higher infection rates than other ducks, geese, and swans 247 . These important species for avian influenza virus transmission all have a relatively high inter-species crossing intensity, which might explain the role of these species in triggering avian influenza outbreaks. However, other alteration, e.g., host species competence and virulence, may affect disease outbreak patterns, depending on the most important host/vector, which could affect disease transmission together with species bridging intensity.

This is a first attempt to map the crossroads for migratory birds. Several issues may bias in the results, which can be improved by further studies including a more systematic movement dataset. The count of connections between two nodes may not necessarily be representative of the probability of bird movements between these two nodes because it can be affected by the probability of both marking and recovery/recapture ${ }^{250}$. For example, the recovery rate may be very high in regions with intensive hunting. The length of hunting season between the two periods defined in this study has been changed, which may result in a different recovery rates in different periods 251. On the other hand, rare species like mergansers were found to have more patchy routes than the common species like mallard (Appendix S6.2), maybe because few of these birds have been ringed and recovered compared to the common species. This may result in a lower inter-species bridging intensity of these rare species (Figure 6.4). Last but not least, as we only quantified spatial overlaps between migration routes, the temporal coexistence and density of these populations/species should be further tested.

To conclude, these migratory species can facilitate virus transmission around the world by contacting other populations/species at crossroads. The changing migratory ranges under rapid climate and land use changes facilitate these contacts between different populations within species and between different species, and subsequently facilitate long-distance virus spread. Both inter-species and intra-species crossing patterns pose high risk for HPAIV transmission, offering potential for targeted disease surveillance at these crossroads, especially of bridging species. By providing quantitative details of the spatial distribution of intra-species crossroads and the importance of a bridging species, our results give insights for early warning strategies in an effort to prevent HPAIV panzootics. Further 
knowledges about the spatio-temporal variations in marking and recovery/re-sighting probability could assist for more precise understandings of these crossroads.

\subsection{Acknowledgements}

We thank the Bird Banding Laboratory of Patuxent Wildlife Research Center of US Geological Survey, the Bird Banding Office of the Canadian Wildlife Service, and all the national ringing schemes involved in the Euring data project. Special thanks to Danny Bystrak (US Geological Survey) and Dorian Moss (Euring) for managing our data applications and preparing the dataset for this study. We thank all the people who assisted in collecting and organizing the marking and recovery/re-sighting data. We thank Jente Ottenburghs (Wageningen University) for his insightful comments on the manuscript. Financial support was provided by Chinese Scholarship Council (No.201600090128). We declare no competing interests. 


\section{Supplementary information}

Appendix S6.1 Studied species list and their inter-species crossing intensity value for bridging (bridging intensity) and being bridged (focal intensity) by other species in North America. The species were ranked from the bridging species with the highest inter-species crossing intensity to the one with the lowest.

\begin{tabular}{|c|c|c|c|c|c|c|}
\hline ID & Scientific name & Common name & $\begin{array}{l}\text { Sample } \\
\text { size } \\
1950- \\
1984\end{array}$ & $\begin{array}{l}\text { Sample } \\
\text { size } \\
1985- \\
2019\end{array}$ & $\begin{array}{l}\text { Bridging } \\
\text { intensity }\end{array}$ & $\begin{array}{l}\text { Focal } \\
\text { intensity }\end{array}$ \\
\hline 20 & $\begin{array}{l}\text { Anas } \\
\text { platyrhynchos }\end{array}$ & Mallard & 601353 & 661921 & 14450461 & 23928 \\
\hline 9 & $\begin{array}{l}\text { Branta } \\
\text { canadensis }\end{array}$ & Canada Goose & 326687 & 949080 & 2194326 & 579467 \\
\hline 10 & Aythya valisineria & Canvasback & 20344 & 5899 & 1321271 & 8164 \\
\hline 24 & Aythya americana & Redhead & 48201 & 12832 & 586172 & 189432 \\
\hline 16 & Anser albifrons & $\begin{array}{l}\text { Greater White- } \\
\text { fronted Goose }\end{array}$ & 12874 & 20096 & 565241 & 7914 \\
\hline 27 & Chen caerulescens & Snow Goose & 75768 & 64889 & 318474 & 14241401 \\
\hline 14 & Mareca strepera & Gadwall & 7691 & 18276 & 293866 & 50581 \\
\hline 1 & Anas rubripes & $\begin{array}{l}\text { American Black } \\
\text { Duck }\end{array}$ & 131794 & 46187 & 282037 & 16201 \\
\hline 5 & Anas discors & Blue-winged Teal & 49876 & 86612 & 207515 & 188130 \\
\hline 17 & Anas crecca & Eurasian Teal & 36736 & 46764 & 174006 & 104726 \\
\hline 22 & Anas acuta & Northern Pintail & 129563 & 39453 & 173311 & 611382 \\
\hline 31 & Aix sponsa & Wood Duck & 71758 & 159542 & 134266 & 84643 \\
\hline 2 & Mareca americana & American Wigeon & 24043 & 5954 & 64825 & 332382 \\
\hline 19 & Aythya affinis & Lesser Scaup & 25792 & 7733 & 45398 & 2209146 \\
\hline 23 & Spatula clypeata & $\begin{array}{l}\text { Northern } \\
\text { Shoveler }\end{array}$ & 4132 & 1851 & 24914 & 7970 \\
\hline 25 & Aythya collaris & $\begin{array}{l}\text { Ring-necked } \\
\text { Duck }\end{array}$ & 16823 & 8450 & 17808 & 2068484 \\
\hline 29 & Cygnus buccinator & Trumpeter Swan & 397 & 9998 & 17099 & 63232 \\
\hline 13 & $\begin{array}{l}\text { Bucephala } \\
\text { clangula }\end{array}$ & $\begin{array}{l}\text { Common } \\
\text { Goldeneye }\end{array}$ & 1632 & 1479 & 15085 & 7920 \\
\hline 30 & $\begin{array}{l}\text { Cygnus } \\
\text { columbianus }\end{array}$ & Tundra Swan & 4340 & 4860 & 9791 & 16848 \\
\hline 7 & Bucephala albeola & Bufflehead & 1672 & 716 & 9345 & 2355 \\
\hline 18 & $\begin{array}{l}\text { Lophodytes } \\
\text { cucullatus }\end{array}$ & $\begin{array}{l}\text { Hooded } \\
\text { Merganser }\end{array}$ & 296 & 1947 & 4836 & 85180 \\
\hline 11 & Anas cyanoptera & Cinnamon Teal & 1468 & 1572 & 639 & 5126 \\
\hline 6 & Branta bernicla & Brent Goose & 9941 & 15682 & 383 & 4256 \\
\hline 21 & Anas fulvigula & Mottled Duck & 1720 & 15035 & 269 & 210 \\
\hline 15 & Aythya marila & Greater Scaup & 5487 & 475 & 158 & 399 \\
\hline 12 & $\begin{array}{l}\text { Somateria } \\
\text { mollissima }\end{array}$ & Common Eider & 1496 & 7014 & 83 & 1619 \\
\hline
\end{tabular}


Appendix S6.2 Studied species list and their intersection value for bridging and being bridging by other species in Eurasia

\begin{tabular}{|c|c|c|c|c|c|c|}
\hline ID & Scientific name & $\begin{array}{l}\text { Common } \\
\text { name }\end{array}$ & $\begin{array}{l}\text { Sample } \\
\text { size } \\
1950- \\
1984\end{array}$ & $\begin{array}{l}\text { Sample } \\
\text { size } \\
1985- \\
2019\end{array}$ & $\begin{array}{l}\text { Bridging } \\
\text { intersection }\end{array}$ & $\begin{array}{l}\text { Focal } \\
\text { intersection }\end{array}$ \\
\hline 1840 & Anas crecca & Eurasian Teal & 27164 & 70146 & 167188 & 8422 \\
\hline 1860 & $\begin{array}{l}\text { Anas } \\
\text { platyrhynchos }\end{array}$ & Mallard & 150009 & 98711 & 146205 & 6154 \\
\hline 1570 & Anser fabalis & $\begin{array}{l}\text { Taiga Bean } \\
\text { Goose }\end{array}$ & 2085 & 19749 & 19325 & 18265 \\
\hline 2030 & Aythya fuligula & Tufted Duck & 19699 & 25781 & 17603 & 36851 \\
\hline 1590 & Anser albifrons & $\begin{array}{l}\text { Greater } \\
\text { White-fronted } \\
\text { Goose }\end{array}$ & 2199 & 38780 & 14094 & 27768 \\
\hline 1540 & Cygnus cygnus & $\begin{array}{l}\text { Whooper } \\
\text { Swan }\end{array}$ & 533 & 31976 & 11720 & 15287 \\
\hline 1790 & Mareca penelope & $\begin{array}{l}\text { Eurasian } \\
\text { Wigeon }\end{array}$ & 10387 & 8986 & 10228 & 196408 \\
\hline 1520 & Cygnus olor & Mute Swan & 125838 & 474359 & 6334 & 6620 \\
\hline 1610 & Anser anser & Greylag Goose & 10565 & 171934 & 3539 & 7794 \\
\hline 1980 & Aythya ferina & $\begin{array}{l}\text { Common } \\
\text { Pochard }\end{array}$ & 8406 & 13762 & 3518 & 68869 \\
\hline 1530 & $\begin{array}{l}\text { Cygnus } \\
\text { columbianus }\end{array}$ & Tundra Swan & 348 & 2507 & 2920 & 1275 \\
\hline 1940 & Spatula clypeata & $\begin{array}{l}\text { Northern } \\
\text { Shoveler }\end{array}$ & 5238 & 1734 & 2376 & 940 \\
\hline 1820 & Mareca strepera & Gadwall & 3109 & 4382 & 1460 & 1212 \\
\hline 1660 & $\begin{array}{l}\text { Branta } \\
\text { canadensis }\end{array}$ & Canada Goose & 10181 & 85991 & 1019 & 5049 \\
\hline 1890 & Anas acuta & $\begin{array}{l}\text { Northern } \\
\text { Pintail }\end{array}$ & 8561 & 3409 & 782 & 1132 \\
\hline 1580 & $\begin{array}{l}\text { Anser } \\
\text { brachyrhynchus }\end{array}$ & $\begin{array}{l}\text { Pink-footed } \\
\text { Goose }\end{array}$ & 13189 & 15913 & 504 & 389 \\
\hline 1670 & Branta leucopsis & $\begin{array}{l}\text { Barnacle } \\
\text { Goose }\end{array}$ & 2821 & 30305 & 471 & 6243 \\
\hline 1730 & Tadorna tadorna & $\begin{array}{l}\text { Common } \\
\text { Shelduck }\end{array}$ & 4017 & 9185 & 385 & 790 \\
\hline 2180 & $\begin{array}{l}\text { Bucephala } \\
\text { clangula }\end{array}$ & $\begin{array}{l}\text { Common } \\
\text { Goldeneye }\end{array}$ & 3568 & 12117 & 298 & 318 \\
\hline 1680 & Branta bernicla & Brant Goose & 231 & 2661 & 210 & 186 \\
\hline 2230 & $\begin{array}{l}\text { Mergus } \\
\text { merganser }\end{array}$ & $\begin{array}{l}\text { Common } \\
\text { Merganser }\end{array}$ & 1837 & 2731 & 83 & 290 \\
\hline
\end{tabular}


Appendix S6.3 The migration routes for the studied species in North America. Each colour represents a different migration route identified by a module detection approach.
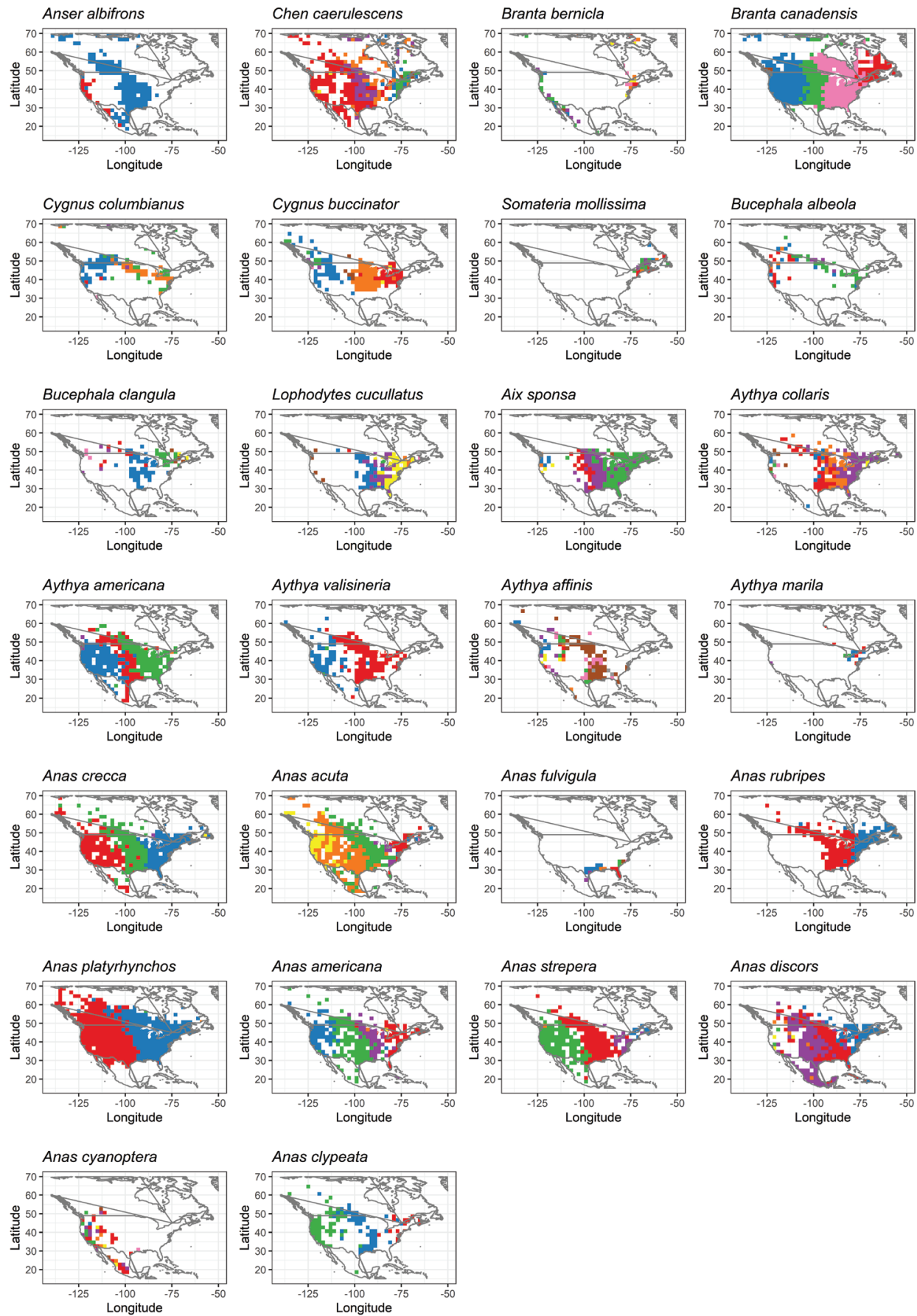
Appendix S6.4 The migration routes for the studied species in Eurasian. Each colour represents a different migration route identified by a module detection approach.
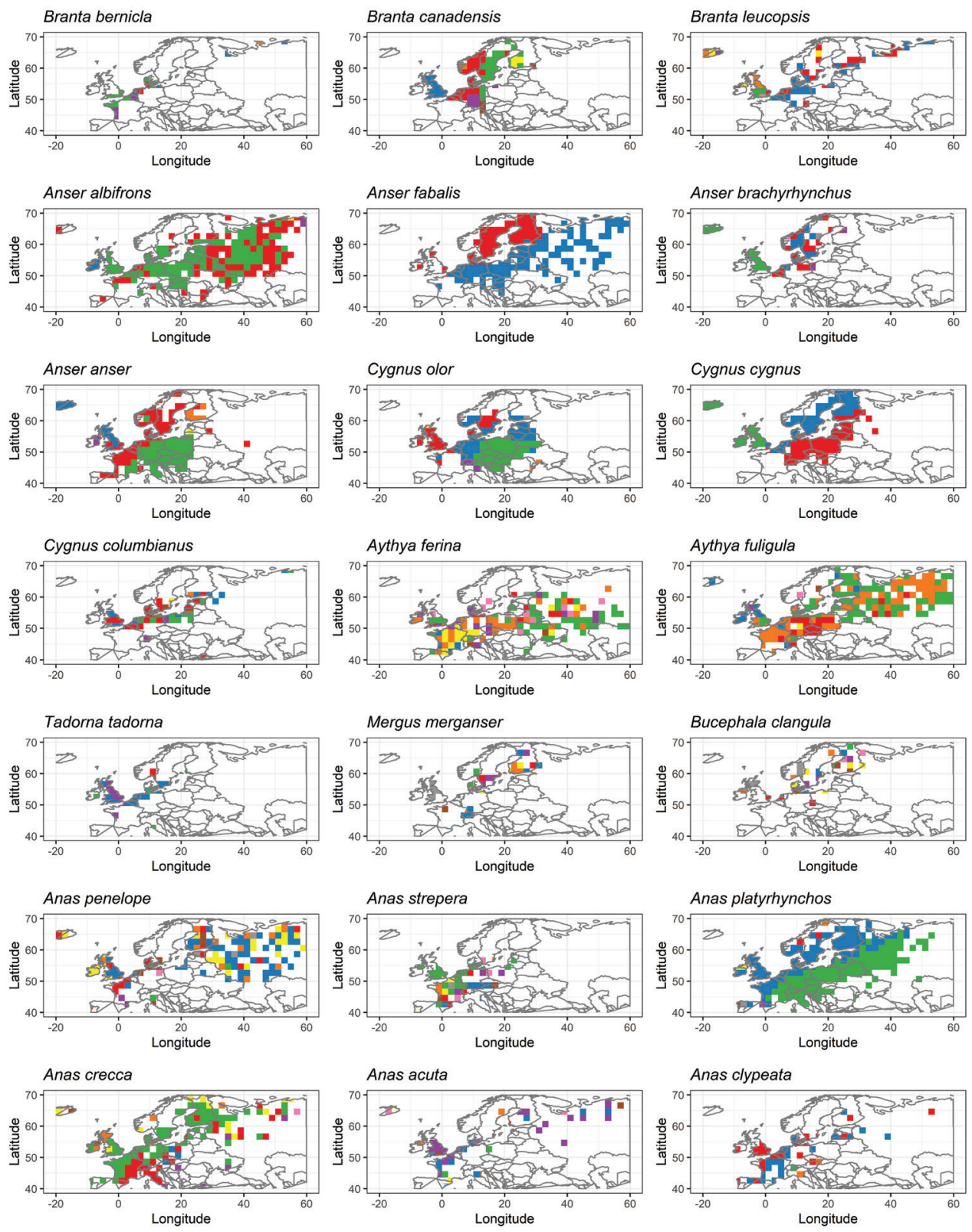
Figure S6.5 Inter-species crossroads. The species pair count is the number of species pair combinations observed in each $200 \times 200 \mathrm{~km}$ grid cell in period 1985 2019. The count is associated with species richness in the grid cell.

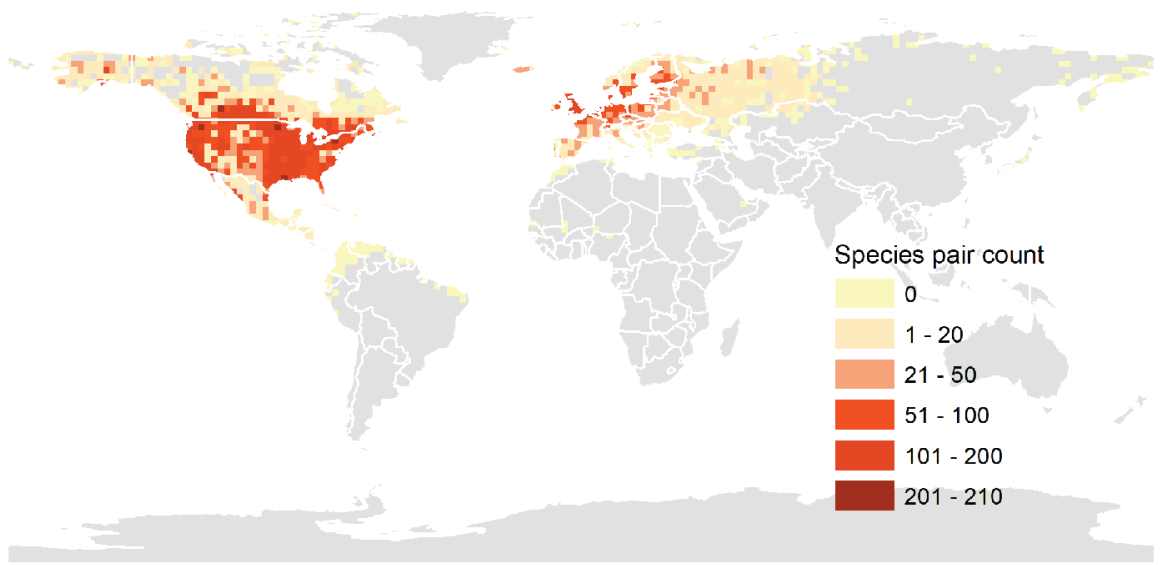

Figure S6.6 Global wetland coverage in (a) 1992 and (b) 2015 and (c) wetland changes from 1992-2015. The percentage of wetland cover is calculated in each $200 \times 200-\mathrm{km}$ grid cell using the global land cover maps from the European Space Agency Climate Change Initiative (ESA CCI).
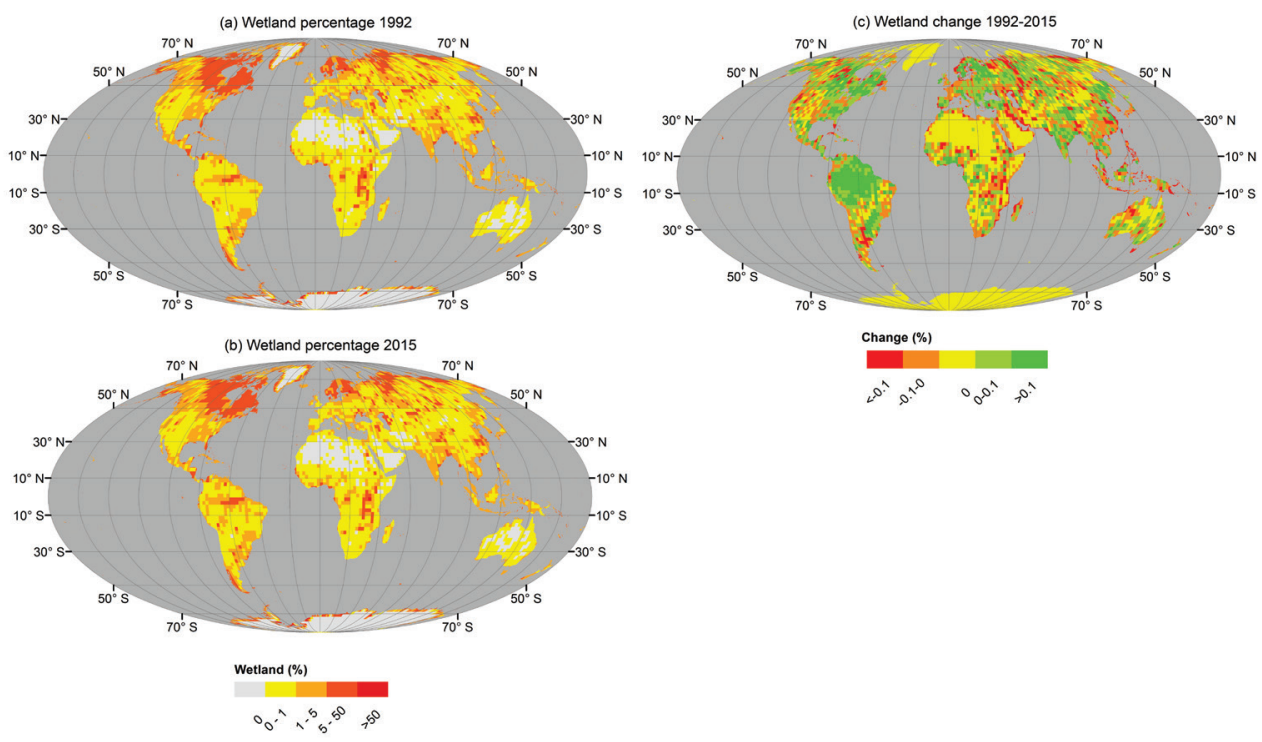
CHAPTER 7 


\section{Synthesis}




\section{Foreword}

I used to play Labyrinth. I like them because the key to win is not fighting, not brute forces, but wisdom and faith to survive. It is as if migratory birds play similar games twice a year. However, for them it is not a game, there are no second chances and there is no turning back. They evolved to be great 'players' but they may start losing this 'game' due to the rapid changes in the maze which is not a playful labyrinth, but a patchwork of suitable habitats connected by flightpaths. The costs of failure are fatal, both for these 'players' and for us who change the rules. 
In this thesis, I applied long-term observations on large-scale movements by birds to illustrate how global changes can influence the network dynamics of migratory birds, potentially leading to population declines (Chapter 3), and rapid spread of diseases by avian hosts (Chapter 6). Integrating these five different chapters, I here propose a practical methodological framework for setting priorities in conservation of migration networks at a variety of scales: a local scale (Chapter 4), a flyway scale (Chapter 5), and at species level (Chapter 2).

\subsection{Multiple consequences of dynamics of bird movement networks under rapid global change}

Birds are excellent bioindicators of ecosystem health under habitat disturbance 58 . Migratory birds use a network of suitable habitat sites 169 . These sites can function as stepping stones for further movement 57 , foraging sites for refuelling ${ }^{68,81}$, but can also facilitate virus exchange (Chapter 6) 60,252, and the exchange of social information 253-257. In an era of rapid global change, disturbance to natural habitats currently poses one of the greatest threats to wildlife ${ }^{48,49}$. Habitat loss from a movement network can potentially cause the network to breakdown rapidly $37,170,252$.

Consequently, a decreasing connectivity in movement network induces low robustness of bird populations in terms of e.g., low migration success, low breeding success, and low resistance and resilience against disturbances and environmental changes. This can subsequently lead to population declines 42,169 , and can reduce the ecosystem services (e.g., seed dispersal) supplied by these birds. Here, I list these different functional aspects of consequences from decreasing connectivity in bird movement networks.

\section{Information diffusion}

Information transmission among individuals critically affects animal population sustainability, e.g., in terms of the efficiency of foraging behaviour and the suitability of migration strategies 225,258 . For instance, a majority of European bee-eaters (Merops apiaster) stay together in groups during their 14,000-km migration using coordinated migration and foraging strategies 209 . Individual barnacle geese (Branta leucopsis) which follow social information acquire food more frequently, compared to those ignore the information 257. Similarly, Aplin et al. ${ }^{28}$ found that food-related social information was rapidly diffused by individual great tits (Parus major), and this allowed foraging innovations to persist over generations. The movement 
of individuals across the landscape is one of the key factors facilitating the social transmission of information among birds ${ }^{253}$. Indeed, the ability for individuals to move between sites within a population shapes its social structure ${ }^{24}$, and consequently any changes which affect movement may also influence the effectivity and efficiency of information transmission. Further research is needed for better understanding the extent to which decreasing connectivity in bird movement networks may impede information spread among wild bird populations.

\section{Predator effect}

The survival and reproduction success of bird species are strongly affected by their predators 259-261. For instance, greylag geese (Anser anser) utilized suboptimal foraging patches and constrained their home ranges during moulting to avoid high predator risks ${ }^{262}$. Voelkl et al. ${ }^{260}$ experimentally found that a single nonlethal 'attack' by a model sparrow hawk (Accipiter nisus) triggered an instantaneous and extensive turnover in flock composition of great tits and blue tits (Cyanistes caeruleus). A fragmented landscape affects animal movements, and disturbance to particular areas may cause individuals to make non-optimal anti-predatory decisions ${ }^{263}$, e.g., higher social mixing and suboptimal habitat use. In highly fragmented landscapes, even human-induced predator management strategies cannot mitigate the population declines of prey species under predator attacks 263 . However, a fragmented movement network of a prey species can also be suboptimal for a predator species 264,265 , and thereby decrease the predation risk for a prey species. Despite various studies covering effects of habitat configuration on predator-prey interactions, few studies have tested this specific aspect explicitly and empirically 266 . The mechanism of how connectivity loss in bird movement networks affects population dynamics of prey species remains unclear, which may be elucidated by further studies.

\section{Disease transmission}

Many bird species are competent hosts for various infectious pathogens 216,267, e.g., Usutu virus, West Nile virus, and avian influenza viruses.

Because of their high mobility, the movement of birds is an important agent for large-scale dispersal of viruses ${ }^{60}$. Although bird movements may be impeded through habitat disturbance, reducing mobility and network efficiency, this can also facilitate virus transmission if infected birds move to novel areas in response to a perturbation effect 268 . This effect was observed among badgers (Meles meles) and red foxes (Vulpes vulpes), which expanded 
their home ranges and invaded novel regions in response to culling operation for Bovine tuberculosis 268 and rabies 269,270. Moreover, recent patterns of site loss in bird migration networks may increase the probability that birds from different flyways co-occur more intensively at their crossroads (Chapter 6), and thereby increase the probability of disease infection in these birds, also by restricting their movements in smaller spatial ranges ${ }^{252}$. However, habitat perturbations and partial network collapse can also lead to reduced population sizes, which can have a negative effect on disease spread and pathogen infection probabilities. It is therefore pivotal to better understand how habitat perturbations and site loss affect movement networks, for better understanding of disease transmissions by networks of these mobile animals.

\section{Seed dispersal}

Bird species are highly mobile. Many species, e.g., Anatidae, are herbivorous and feed often on plants and their seeds. Various plant seeds can survive during gut passages in birds 271,272 , and thus the short and long distance movements of birds are one of the primary agents for seed dispersal between isolated habitat patches ${ }^{273}$. For instance, mallards (Anas platyrhynchos) can disperse seeds farther when using fragmented movement networks 274 because less connected habitat patches may force them to move farther for foraging and resting. In contrast, a higher diversity of plant seeds can be transported in well-connected mallard movement networks, because the survival rates of smaller seeds in mallards' gut significantly decreased when travelling over long distances ${ }^{274}$. Therefore, connectivity loss in movement networks may force wild birds to disperse seeds over longer distances, but the diversity of dispersed seeds could decrease due to heterogeneous survival times of seed species inside a bird.

\section{Gene flow}

Changing structures in bird movement networks may affect population genetic structures and subsequently influence genetic evolution in birds ${ }^{275}$. For instance, shifted migratory patterns in response to environmental changes induce a higher mixing rate between different populations of barnacle geese, thus, an increased chance of genetic exchange was observed between these populations ${ }^{276}$. Moreover, the probability of avian hybridizations may be altered ${ }^{277}$, because inter-species mixing based on structures of movement networks is a prerequisite for mating with a different species. Thus, linking spatial-temporal movement patterns with 
phylogenetic networks of birds ${ }^{278}$ may facilitate better understanding of evolutionary problems in birds.

\section{Cascading effect}

These different aspects of functional degradations caused by connectivity loss of bird movement networks are dependent on each other, and may lead to species declines, local extinctions, and cascading changes in ecosystems. In Chapter 3, I observed a population decline in waterfowl species with decreasing connectivity in their migration networks ${ }^{169}$. Meanwhile, Studds et al. ${ }^{35}$ found that habitat loss in a keystone site, i.e., Yellow Sea mudflats, induced rapid population declines in migratory shorebirds. This can be explained by a simulation study that showed that losing the coastal sites in Bohai Bay leads to the collapse of stork migration networks, and completely disables their migration success ${ }^{37}$. Different agents work together in between, as for instance, decreasing connectivity in movement network may impede social information spread among birds, which can induce a reduced feeding efficiency. Reduced energy intake rate may result in a poorer predator escape due to reduced condition, and hence flight ability ${ }^{279}$. Subsequently, a stronger impact of predators on prey species can turnover their social structure 260 , and, again, as a feedback, impedes information spread, leading to a lower feeding efficiency and higher predation risk. Moreover, an increased mixing may facilitate disease transmission.

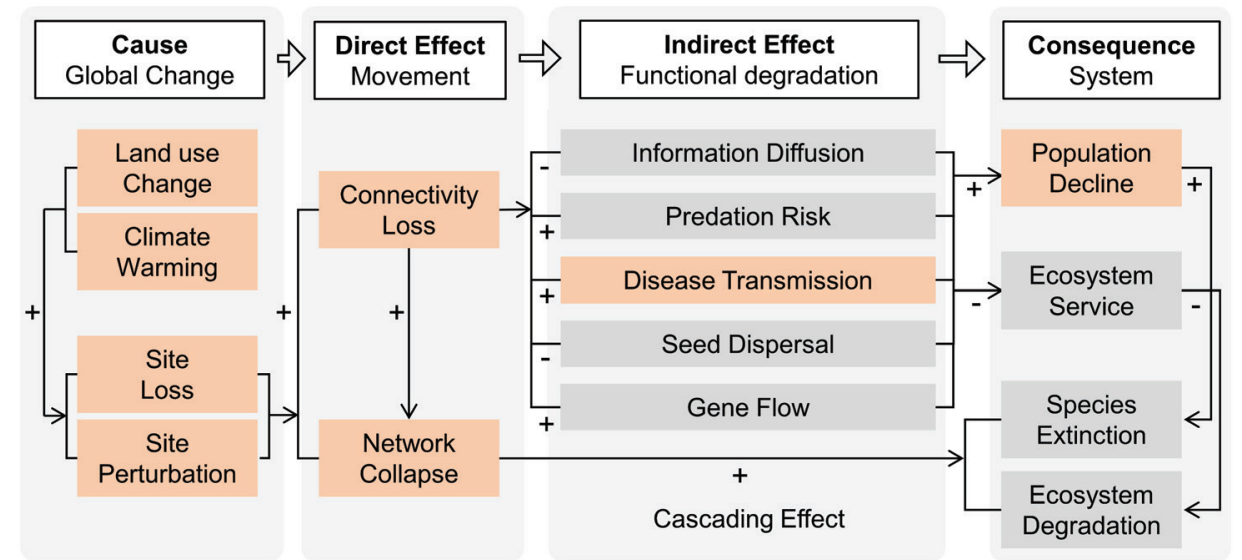

Figure 7.1 Causes and hypothesized effects of changing connectivity of bird movement networks under rapid global changes. The blocks in red were covered in this thesis, while the ones in grey were not covered. "+" represents a potential positive effect, while "-" is a negative one. These relationships illustrate the various hypotheses, and need to be further investigated. 
The effect of site loss on bird populations has been quantified by multiple modelling studies $32,37,170$, which simulated site loss by taking out sites and their connections within the network (Chapter 5). However, the consequence of site loss can be overestimated, because the resilience of the bird movement networks is not taken into account. A relevant example of the influence of resilience comes from studies for social networks of wild birds. Previous social network studies have used simulations to propose that removing individuals will reduce social connectivity. Firth et al. 30 experimentally showed that new social ties formed among the remaining individuals immediately after removing an individual from a bird social network. These new social ties maintained the connectivity of the social network, indicating the strong resilience of the network (contrary to the predictions of simulation studies). This can also be the case in a bird movement network, because birds may establish new movement pathways or make use of novel sites when a site is disturbed or when new sites are added to the network 50,225 .

Therefore, the simulation-based predictions of how bird populations respond to habitat degradation may be partly inaccurate and conceptually incomplete, and should be further validated by field experiments. To empirically quantify the functional degradation (i.e., connectivity and associated consequences such as information diffusion, predation risk, disease transmission, seed dispersal, gene flow, and cascading effects), we need to build a field experimental system introducing experimentallyimposed disturbance within a movement network, and study not only the collapse effects, but also the resilience of the network by building new (spatial) connections.

\subsection{Actions to cope with the dynamics: policy making for prioritizing site conservation}

Conserving a network of sites for bird movements may contribute to the conservation of particular species but also to the ecosystem services provided by their movements or these species. To efficiently maintain or restore the ecological functions of bird movement networks, it is critical to prioritize conservation efforts for keystone sites that have a crucial contribution to the connectivity of the entire network. This has been pointed out and investigated extensively since scientists 18 years ago ${ }^{280}$. However, this specific aspect has not yet been included in global conservation actions 
for birds, according to my review of existing criteria of international conservation policies for defining a site as important, and hence for setting conservation priorities (Table 7.1). I categorized their criteria for site importance for birds into the following aspects.

Table 7.1 International policies of site importance for bird conservation included in this review. Other international efforts include the Critical Site Network Tool (CSN, Wetlands International) and Birds Directive - Designation of the Special Protection Areas (SPA, Natura 2000). Because CSN applied criteria of IBA and Ramsar and SPA suggested margin of discretion by countries, they were not reviewed explicitly. Other flyway conventions are also relevant, e.g., Agreement on the Conservation of African-Eurasian Migratory Waterbirds (AEWA), the East Asian-Australasian Flyway Partnership (EAAFP), and Migratory Bird Treaty Act (MBTA). They were not reviewed explicitly either, because their documents emphasized the criteria for species importance in the flyway instead of those for site importance.

\begin{tabular}{llll}
\hline Abbreviation & Name & Source & Policy maker \\
\hline GIBA & Global Important Bird Area & BirdLife & BirdLife \\
& & International & International \\
EIBA & European Important Bird & BirdLife & BirdLife \\
& Area & International & International \\
MIBA & Middle East Important Bird & BirdLife & BirdLife \\
& Area & International & International \\
Ramsar & The Ramsar Sites Criteria & $\begin{array}{l}\text { Ramsar } \\
\text { Convention }\end{array}$ & Ramsar Convention \\
ASCI & Areas of Special & Bern Convention & Council of Europe \\
& Conservation Interest & & \\
WHSRN & Western Hemisphere & WHSRN Executive & Manomet Inc. \\
& Shorebird Reserve Network & Office & Audubon Society \\
& & & Wetlands \\
& & & International \\
& & & Wildlife Habitat \\
& & Canada \\
WCASN & Western/Central Asian Site & Convention on & UN Environment \\
& Network & Migratory Species & \\
\hline
\end{tabular}

\section{Diversity}

A majority of the criteria are formulated from the a diversity perspective, which are quantified by the presence of threatened or restricted-range species. These are included in most of the reviewed policies, i.e., GIBA (referred to Articles A1 and A2 in the policy), MIBA (Articles B2 and B3), EIBA (Articles B2, B3, C6), Ramsar (Criterion 2), ASCI (Articles a and b). The bird species which are categorized as Critically endangered, Endangered, Vulnerable in IUCN Red List Categories are defined as vulnerable in GIBA and Ramsar. For EIBA and MIBA, the threatened species are those that have an unfavourable conservation status at a regional level, i.e., in Europe and the Middle East. Restricted-range species are those species of which a majority of their population distribute in the focal site. Additionally, ASCI (b) listed sites supporting significant numbers of species, namely with a high 
species richness, as areas in need of special conservation actions. WCASN (Criterion 1) specifically defines sites hosting Siberian cranes (Leucogeranus leucogeranus) or potentially providing habitats for them as important.

\section{Population size}

Many of the criteria are related to the number of birds using the focal site, which are quantified by either relative or absolute abundance of the different bird species. For relative abundance, regularly holding $\geq 1 \%$ of the global/flyway/European population of bird species is included in GIBA (A4), EIBA (B1,C2,C3), MIBA (B1), WCASN (Sub-qualifier 2.3), and Ramsar (Criterion 6). WHSRN (2) defines sites hosting at least 30\% (hemispheric level), $10 \%$ (international level), or 1\% (regional level) of the biogeographic population of one shorebird species as important. ASCI (b) mentioned that an area supporting important populations of a species is important for conservation. Although beyond the review scope, at country level, the relative abundance within a country is sometimes used as a country-level criteria, e.g., for the UK (bto.org). For the absolute abundance of a bird species, a site regularly holding $\geq 20,000$ waterbirds/seabirds/shorebirds is included in EIBA (C4), WHSRN (1), WCASN (Sub-qualifier 2.1) and Ramsar (Criterion 5). A site holding $\geq 25$ migratory cranes is included in WCASN (Qualifier 1). Moreover, WCASN (Sub-qualifier 2.2) combines criteria of relative and absolute abundance, which defines a site as important when $\geq$ $0.25 \%$ of a migratory waterbird population or $\geq 5,000$ waterbirds were recorded during a single count.

\section{Habitat property}

There are two criteria about habitat properties in Ramsar (Criteria 1 and 3) and two in ASCI (c and d), but all of them are qualitative criteria. A site is defined as important when it contains a special/representative/endangered habitat type or includes important plant or animal species for maintaining a particular ecosystem function. The sites holding range-restricted species may provide unique habitats for these species, thus, the corresponding criteria (explained in section a) are also relevant to this category.

\section{Movement network}

Although quantitative measures with regard to a movement network are not included in the reviewed criteria, some of them are relevant to the connectivity of bird movement networks. EIBA (B1) and MIBA (C5) both defined the 'bottleneck' sites holding $\geq 3,000$ cranes/raptors or $\geq 5,000$ storks during their migration as important bird areas. ASCI (e) defined 
important areas for migratory species as special targets for conservation. Additionally, Ramsar (Criterion 4) and WCASN (Sub-qualifier 2.4) define a site as important when it holds animals at a critical stage of their life cycles during adverse conditions, for example an important stepping-stone site during bird migration.

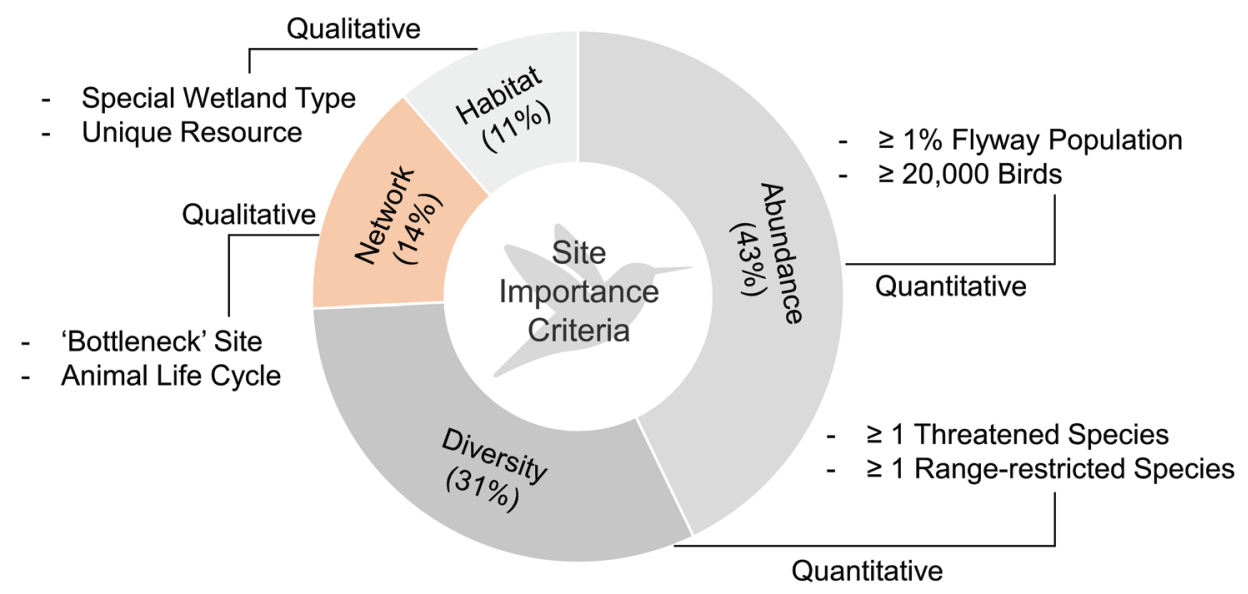

Figure 7.2 A summary of the criteria from international policies for defining the importance of a site for bird conservation. The proportion of reviewed criteria categorized into four classes was calculated respectively.

Most of the reviewed criteria (85\%) focus on site-specific characteristics of habitat areas. Although the majority of the criteria (69\%) are quantitative, the quantitative criteria are all about bird abundances and species at a site. All five criteria (14\%) relevant to bird movement networks are at a qualitative level (Figure 7.2). Moreover, four of the five criteria are regional for Europe, Western/Central Asian, and Middle East, and two are species-specific criteria which are formulated specifically for storks, raptors, and/or cranes.

Although quantitative and explicit criteria regarding bird movement networks have not been included in international policies yet, recent efforts have been made to propose this idea for setting priorities for bird conservation, especially for migratory birds. The Convention on the Conservation of Migratory Species of Wild Animals (CMS) has strongly emphasized the importance of the connectivity of movement networks for conservation of migratory animals in CMS Resolution 12.7 and CMS Resolution 12.26 197. The Aichi Biodiversity Targets from the Convention on Biological Diversity (CBD) included maintaining well connected systems of protected areas for terrestrial and marine animals in Target 11 for Strategic Goal C (cbd.int/sp/targets/). Moreover, country-level protected site 
networks (e.g., British Trust for Ornithology, BTO) and international agreements on bird conservation (e.g., Agreement on the Conservation of African-Eurasian Migratory Waterbirds, AEWA) also promoted the idea of conserving birds from a network perspective. And thus, a quantitative methodological framework may facilitate the implementation of these proposed ideas regarding to maintaining connectivity of bird movement networks in protection measures.

\subsection{Setting up a framework for conservation decisions for movement networks}

To further facilitate policy making for conserving birds from a network perspective, I propose a quantitative framework for the definition of site importance in a bird movement network in this section. The loss of a site from a migration network can have no effect on a species, or it can contribute to a rapid local extinction of the species (Chapter 5) ${ }^{93}$. The importance of a site depends not only on its local quality as habitat for a specific bird species (Chapter 4), but also on its contribution to the connectivity of that species' movement network (Chapter 5). Prioritizing site conservation efforts should take the site's position in the bird movement network into account (Chapter 5). Millions of birds are being watched (citizen science data), tracked, banded, or ringed each year, providing a large number of occurrence and movement data, which gives opportunity for quantitatively identify keystone sites in a bird species' movement network. Thus, the proposed framework goes through the following steps.

The first step is to construct an empirical bird movement network According to network theory, a network, regardless of its type, consists of nodes and edges between the nodes (Box 1.1 in Chapter 1). For bird movement networks, nodes are the sites where birds land to rest, breed or forage; edges are their flights between a pair of sites. In this thesis, I incorporated several approaches to quantifying nodes and edges in a bird movement network (Chapter 2-6), which I summarize along with other relevant studies as follows.

Identify nodes. There are two main approaches to identify the nodes within a bird movement network. The nodes can be defined as areas which birds intensively utilized based on their movement trajectories (Approach N1, Chapter 4, Chapter 5). Bird sampling points, such as bird banding and recovery sites, were assumed to be staging areas for birds, and were widely 
defined as nodes in a species' movement network 31,32,281. However, this may be misleading because of potential sampling biases. As for instance, these bird samplings are often carried out during the day, which may not capture the roosting sites and nocturnal feeding sites that intensively used by some species like dabbling ducks. Thus, I recommend to apply home range estimators to bird tracking data, which are often with higher spatial and temporal resolution and are able to capture both diurnal and nocturnal bird movements, to identify core utilized areas as nodes in a movement network. Home range estimators identify areas with intensive animal movements based on spatio-temporal point patterns ${ }^{282}$. Main home range estimators include Maximum Convex Polygons Methods (MCP), Kernel Density Estimators (KDE), and Brownian Bridge Movement Models (BBMM), among which dynamic BBMM and movement-based KDE were proved to be the most accurate estimators for home range definition ${ }^{282}$. Chapter 5 used dynamic Brownian Bridge Movement Models (dBBMM) for identifying stopover sites utilized by migratory birds which were found to be similar with the stopover sites identified by the Guéguen method in Chapter 3. The Guéguen method (Guéguen, 2001) is a novel alternative which used a Bayesian division algorithm to classify animal behaviour according to turning angles and step lengths, which can be an equally good tool for node identification (Chapter 4).

The nodes can also be identified by classification of potential sites which are suitable for the focal species to rest, breed, or forage, based on relevant environmental factors (Approach N2, Chapter 2, Chapter 4). The simplest way is to identify areas, classified as a suitable land cover class for focal species, e.g., wetland patches for waterbirds ${ }^{44}$ and forest patches for woodpeckers ${ }^{33}$. Some studies define protected areas as nodes within a movement network to measure the efficiency of current conservation efforts for conserving movements of animals 283,284 . However, defining nodes by habitat classification may be biased by missing many other crucial factors that shape a species' distribution, as for instance, fish densities for fisheating birds, and climatic conditions for migratory birds. These factors can be incorporated by performing species distribution models, e.g., Generalized Linear Model (GLM), Maximum Entropy (MAXENT), or Random Forest. Taking advantage of abundant bird occurrence data (e.g., eBird, GBIF, birdtalker) and remote sensing data for environmental monitoring, these models can take most crucial factors shaping a species' distribution into account. Chapter 2 used a GLM 188 to identify the nodes while MAXENT may 
be an alternative because it can generate accurate predictions for species distributions by the machine learning algorithms and no absence data is needed 285 .

Approach N1 identifies utilized sites which were visited by marked birds while Approach N2 identifies all potential sites with suitable environmental conditions. Approach $\mathrm{N} 1$ is accurate but a representative proportion of the focal population is required to be sampled over an extensive period to capture the heterogeneity. As for instance, the defined nodes are sites used by marked individuals but there may be other sites used by the unmarked ones which would not be detected by this approach. Moreover, there are sites currently not used by birds but may be used later when their movement network changes, for example, temporal wetlands in the Mediterranean or Australia may only flood in a minority of years which may not fall inside the surveillance period of the tracked birds. Thus, using Approach N1 may underestimate the dynamics of the bird movement network. Approach N2 can account for this uncertainty by detecting more potential sites based on long-term and fine-temporal-resolution remote sensing data. However, the flexibility of bird species to environmental changes may be overestimated 155 because the ability of birds to explore novel sites remains uncertain. I suggest to combine Approach N1 and N2 when investing the dynamics of movement networks, and also to use longer time windows to quantify these dynamics. Specifically, current bird movement networks can be constructed by utilized sites, while the unused potential sites could be included when modelling the changes in networks, and the distances from utilized sites to unused sites could be taken into account as a proxy for its potential use.

Quantify edges. The edges for a bird movement networks can be directed or undirected. A directed network is more accurate for describing movement patterns of migratory birds that often seasonally move in a certain direction or immigration of resident birds (i.e., long-distance dispersal or range expansion due to environmental changes). When studying resident birds or local movements of migratory birds, an undirected network is suggested so that more network metrics can be included in the calculations (some network metrices, e.g., effective resistance, cannot be calculated for a directed network).

The edges in a bird movement network can be weighted or unweighted. For the unweighted ones, if movements are possible to occur between two 
nodes, an edge forms between them. I suggest to use a weighted network to describe these bird movements. The edges are then weighted by an index which estimates the probability of bird movements between nodes. The following indices can be used to quantify weights for the edges.

The most straightforward quantification for the weight is the count of movements between two sites (Approach W1, Chapter 6) 31,32,46. The more representative the sampled individuals are to the focal population, the closer the distributions of number of movements between sites to that of the movement probability distribution. This approach is suitable for ringing/banding recapture datasets like EURING dataset ${ }^{286}$, banding recovery data from the Bird Banding Laboratory (US Geographical Survey) and the Bird Banding Office (Canadian Wildlife Service), which cover decades of data for a large number of birds. This approach also works for high-resolution datasets of local bird movements like from the Wytham tit study in which the majority of wild birds in the population are tracked 287.

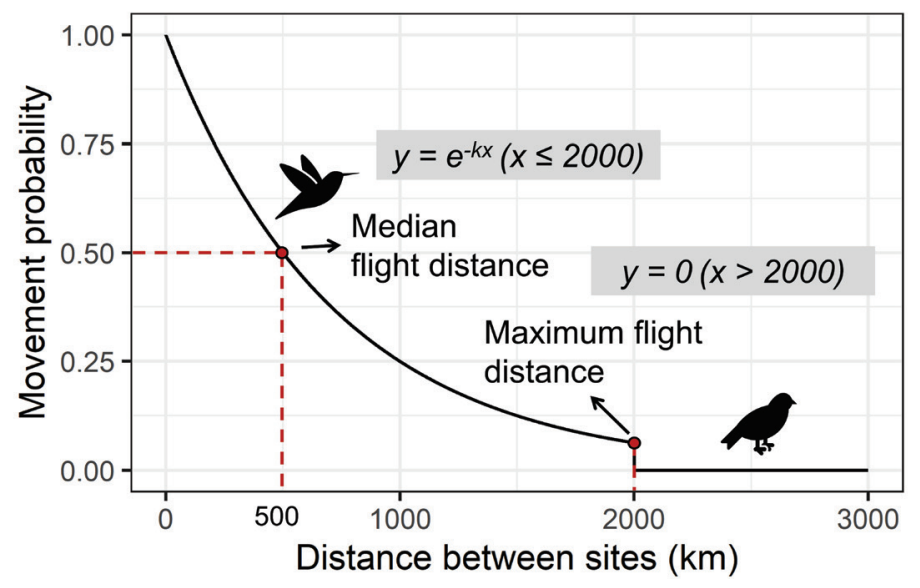

Figure 7.3 Quantifying the probability of dispersing from one site to the another by a decreasing exponential function of distances ${ }^{146}$. The probability is fixed to be zero when the distance between sites exceeds the birds' maximum flight ability (i.e., $2000 \mathrm{~m}) \mathrm{k}$ is a constant calculated by the median flight distance of the focal birds. Assuming the probability is $50 \%$ when the distance between sites equals the birds' median flight distance (i.e., $500 \mathrm{~m}), k=-\ln (0.5) / 500=-0.001386294$.

Another empirical approach is weighting the edges in terms of the distribution of bird flight distances and the geodesic distances between sites (Approach W2, Chapter 3, and Chapter 5). A decreasing exponential function of distances ${ }^{146}$, assuming that greater site-to-site distance is associated with increased cost of movements between sites, resulting 
subsequently in a decreased between-sites dispersal probability, is mostly used for weighing edges in movement networks (Figure 7.3) 44,57,284. In addition, for migration/immigration, angles that the bird take to move from one node to another can be added to edge weighting ${ }^{190}$, based on the angle distribution of bird flights and the angles between sites. Approach W2 is suitable for bird tracking data which cover a limited number of individuals but provide precise distances and angle distributions of their movements.

When empirical bird movement data are not available, assumptions regarding the difference in quality of the start node (move from) and the target node (move to) can be used to weight the edges (Approach W3). As for instance, the migration flow quantified by differences between site attractiveness of start and target nodes ${ }^{252}$ can be an index for weighing the edges. Other assumptions, including predation risk or disturbance, forage abundance and/or quality, costs of searching and settling in target nodes 208 , can also be included as components to weigh edges.

In summary, the potential assumptions for this probability of quantifying edge weights are constraint by upper limits of non-stop flights for focal species, energetic costs, migratory/immigration directions, energetic efficiency of settling in the target nodes. This is all dependent of course on the available sample sizes and the variables that have been measured. I suggest to empirically estimate the probability of travelling different distances by the distribution of travelling distances or the count of movements between two sites. However, when using Approach W1 and W2, biases caused by uneven sampling methods should be taken into account e.g., by including sampling effort as part of the weight. Dhanjal-Adams et al. 209 used a combined approach (W1 and W2), which is good example for weighing edges $\left(W_{i j}\right)$ by empirical data:

$$
W_{i j}=N_{j} \times D_{i j} \times A_{i j}
$$

Where $N_{j}$ is the proportion of tracked birds using the target node $\mathrm{j}$ (Approach $\mathrm{W} 1) ; D_{i j}$ is the probability of flight from node $i$ to $j$. This probability is quantified by the distance between them and the distribution of non-stop flight distances of tracked birds (Approach W2), e.g., by decreasing exponential function of distances (Figure 7.3); $A_{i j}$ is the probability of flight according to the angles between the nodes, this component can be probably ignored when studying local movements of birds. 


\section{The second step is to measure the connectivity of the species' movement}

\section{network}

Measuring the connectivity of the species' movement network connectivity enables evaluating the degree to which the landscape facilitates their movements and monitoring/simulating changes in this degree. It is also essential for understanding the degree to which the breakdown of the movement network induces functional degradation and/or population declines of the species. Several network metrics can be used for quantifying the connectivity of a bird movement network.

For an unweighted network, the number of alternative routes between two representative sites (e.g., breeding and non-breeding sites in migration networks) can be used to measure the connectivity (Approach C1). The number of alternative routes is an index for how flexible birds can move in the network, and the degree to which birds can cope with network changes due to e.g., environmental disturbances such as extreme drought events.

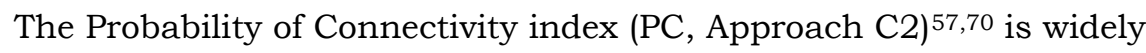
used to quantify functional connectivity in animal movement networks (Chapter 3) 44,284 . PC uses a graph-based algorithm which quantifies the functional connectivity by both the area/suitability of habitat in each node and the probability of movements between nodes, thus, both local habitat availability and connectivity between habitat sites are taken into consideration. This approach requires data on the landscape configuration, e.g., the area of the different habitat classes in each node, which can be measured by analysing land cover maps derived from remote sensing images.

Classic metrics in network theory can also be used to quantify the connectivity of bird movement networks (Approach C3). As for instance, the modularity ${ }^{1}$ of a network, based on classified community memberships ${ }^{8}$, can be used to measure the degree to which the movement network is divided into sub-networks (i.e. modules) located in smaller separate spatial regions ${ }^{32}$. This is important for identifying spatial units for different functional activities of birds, e.g., day roosting and nocturnal feeding of dabbling ducks 288,289 , which is important for targeted conservation efforts. The generalized clustering coefficient algorithm for weighted directed networks ${ }^{6}$ measures the strength of nodes clustering together, which is an alternative to measure the connectivity of local movements of birds ${ }^{31}$. However, relevant ecological hypotheses have to be formulated before using 
these metrics, because they have been designed for other fields, e.g., social science.

Electrical circuit theory for ecological processes 206 is a another promising pathway for measuring the connectivity of bird movement (Chapter 5, Approach C4). This approach introduces effective resistance as a measure for the difficulty of movements in a network, which assumes a higher effective resistance when the landscape impedes the movement between nodes and there are less alternative routes for movements.

Approach $\mathrm{C} 4$ is the optimal model for measuring the degree to which the movement network facilitates bird movements between sites, while Approach C2 considers both site quality and inter-site movements. Both approaches are recommended when data is available. Moreover, calculating these connectivity metrics over temporal networks can provide early warning signals for (full or partial) network collapse. For instance, a movement network is closer to collapse when the number of alternative routes it provided are rapidly decreasing or the effective resistance it provide for the movements of birds is rapidly increasing.

The third step is to rank nodes by their contribution to the connectivity of the movement network

It is important to prioritize conservation efforts for keystone nodes in a movement network with proper management strategies and conservation actions ${ }^{290}$. Several approaches have been used to define the importance of a node by their contribution to the connectivity of the movement networks.

Classic metrics in network theory measure the contribution of a node to the connectivity of its network (centrality), which is suitable for quantifying site importance in movement network (Chapter 5, Approach $\mathrm{S} 1)^{32}$. There are three main measures of node centrality: betweenness, closeness, and degree 15. Degree measures the edges by which a node connects to (out degree) or from (in degree) other nodes ${ }^{4}$, and indicates the degree to which the focal site directly connects to other sites in a movement network. Closeness measures how closely a node connects to other nodes in the network ${ }^{4}$, which indicates the degree to which an animal can easily move from the focal site to other sites. Betweenness measures the shortest paths going through the focal node $4,14,15$, which indicates the degree to which a site functions as an stepping stone in a movement network. Among these metrics, betweenness significantly better captures the keystone sites 
because losing the site with a high betweenness value can result in rapid decreases in the connectivity of a movement network (Chapter 5), thus, I recommend to rank sites by their betweenness values to quantify their importance in a movement network ${ }^{170}$. Besides, other novel node-level metrics may be used, depending on special objectives, as for instance, bridging centrality ${ }^{17}$ is important when measuring crossroads between different flyways or movement modules (Chapter 6).

A node removal process can also measure the centrality of a site in a movement network (Approach S2, Chapter 5). This approach calculates the changes in connectivity of the movement network (Part b) during node removal. A keystone node significantly reduces the network connectivity when it is removed from the system. There are three main methods for site removal: single removal, group removal, and cumulative removal. Single removal removes a site from a bird movement network at one time and quantified site importance by the difference in network connectivity before and after removing it 148 . Group removal removes a group of sites with certain attributes (unprotected sites or sites in the same geographical zone) ${ }^{37}$. Cumulative removal removes sites one by one in a certain order without replacements until the network collapse (e.g., in order of high to low degree of habitat loss; Chapter 5).

Chapter 5 combines approach S1 and S2 by comparing the effect of different orders in terms of values of different node-level network metrics for cumulative removal. For bird migration networks, the betweenness value best captured site importance from a network perspective ${ }^{170}$. For other movement networks, I suggest to use these combined methods to first explore the most suitable network metric explaining the site importance by a node removal process, and then use this selected metric to define importance of a site in the entire movement network.

\section{Implementation}

In summary, this quantitative framework (Figure 7.4) takes advantage of a well understanding of regular patterns of species' movements. Besides birds, this framework may also be appropriate for setting conservation priorities for other terrestrial or marine species ${ }^{284}$, which regularly move between fragments in the landscape or rely on fragmented foraging patches. Furthermore, understanding of other ecological processes based on animal movements (e.g., zoonotic disease transmission and seed dispersal) can also benefit from following this methodology framework. 
The feasibility of this framework relies on the availability of species movement data. Different species, even if for congeneric species (Chapter 2), display different behaviours for movement and habitat use ${ }^{174}$, so I suggest to apply this framework for each species separately in terms of available knowledge about their movement strategies. The feasibility for smaller species may be limited because transmitters tagging on the species should be $\leq 2-4 \%$ of their body weight ${ }^{291}$. Besides, the behaviour of same species may vary considerably in different regions of the world, as for example, wild geese in Europe and North America intensively used agriculture lands 119 while a higher composition of farmlands predicted a lower habitat quality for geese in East Asia (Chapter 4). Thus, availability of information for birds in different flyways or regions also affect the feasibility of this framework.

Putting this quantitative framework into practice will require actions at the intergovernmental levels by working with biodiversity and climate conventions and national level, including in wide range of plans such as National Biodiversity Strategies and Action Plans (NBSAPs) of parties and by mainstreaming these into other development planning, climate change adaptation and mitigation frameworks. In practice, habitat conservation and habitat creation/restoration may be two main improvement strategies for those sites identified as important by this network-based quantitative framework.

Habitat conservation prevents habitat loss by management plans after designating a site as protected area. Designating protected areas is the most used conservation tool which mitigate anthropogenic modification and disturbance for natural habitats by e.g., conservation legislations, hunting restrictions, and regular surveys ${ }^{292}$. These management plans may reduce the construction of barriers (e.g., linear infrastructure, roads, highways, canals, pipelines affect water movement, power lines) to bird movements and key staging areas, which mitigate their mortality due to e.g., solar and wind farms placed at critical migration locations and network sites. However, the effectiveness of this conservation tool may be questionable, as for instance, a very limited proportion of waterbirds was effectively protected by Ramsar Convention, according to the population trends of these birds 293 .

This may be tackled by technical solutions related to habitat creation/restoration, which expand habitat area by creating innovative habitats or restoring destructed or polluted habitats. As for instance, compensating farmers with agricultural subsidies enables wild geese to 
exploit agricultural lands, which are alternative sites in their movement networks ${ }^{186}$. Reflooding of previously drained habitats can also be effective for waterbirds, e.g., winter flooding of rice fields in Japan provided habitats for geese and swans ${ }^{294}$. Invasive predator removal programs restore natural habitats for prey species, which are also evidenced to be effective for species-specific protection for bird populations ${ }^{295}$. Artificial habitat creation like construction of reservoirs and urban parks in keystone areas can be another important technical solution ${ }^{296}$, especially when an additional site is needed for increasing the connectivity of movement networks. However, natural habitat restoration (e.g., water and soil pollution control), which provides higher-quality habitats, may be more effective for connectivity conservation than artificial habitat creation 297.

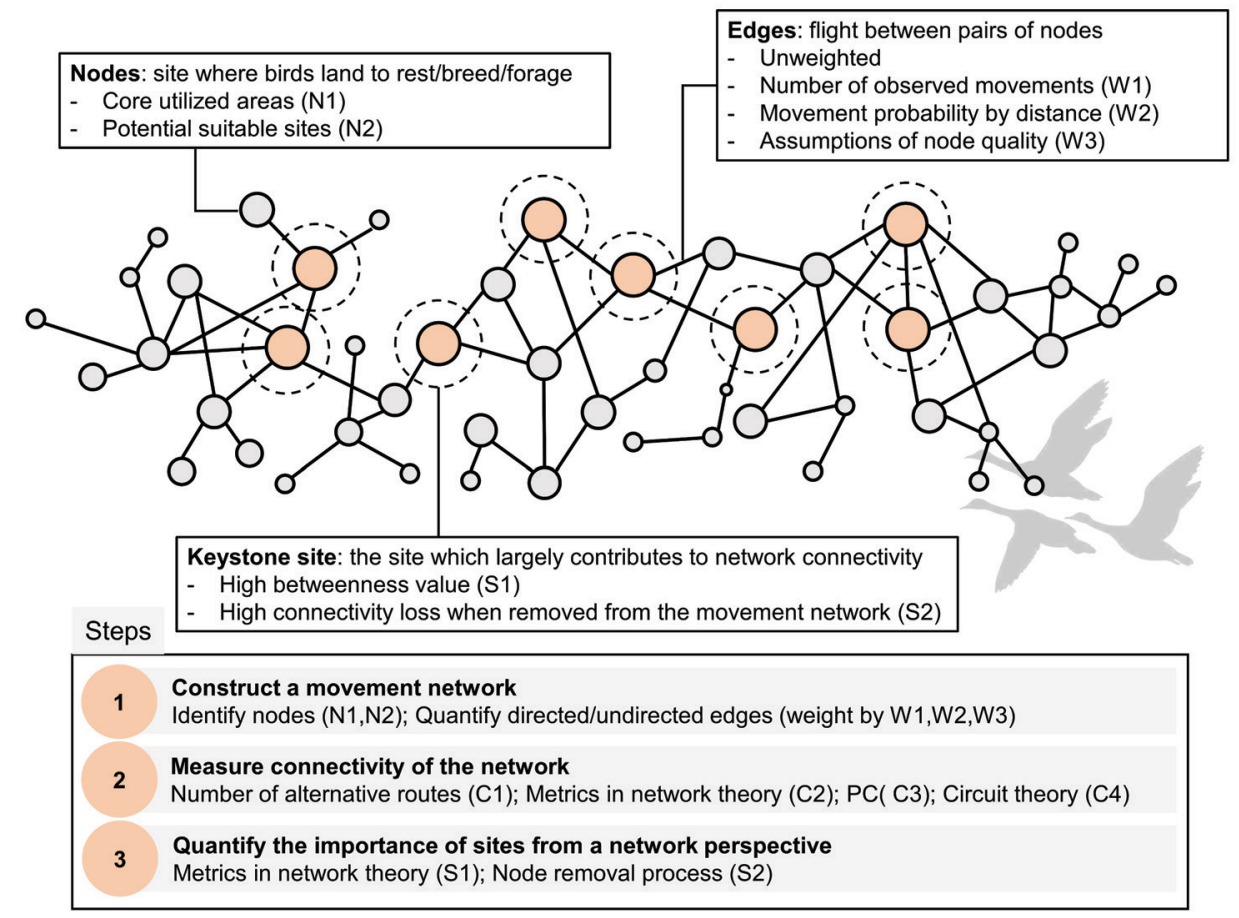

Figure 7.4 Principal components and steps for the proposed framework for conservation decisions for movement networks. The size of nodes represents their importance in the network. 



\section{Bibliography}


1 Newman, M. E. J. Modularity and community structure in networks. Proceedings of the National Academy of Sciences of the United States of America 103, 8577-8582 (2006).

2 Otte, E. \& Rousseau, R. Social network analysis: a powerful strategy, also for the information sciences. Journal of Information Science 28, 441-453 (2002).

3 Faust, K. \& Skvoretz, J. Comparing networks across space and time, size and species. Sociological Methodology 32, 267-299 (2002).

4 Opsahl, T., Agneessens, F. \& Skvoretz, J. Node centrality in weighted networks: Generalizing degree and shortest paths. Social Networks 32, 245251 (2010).

5 Chen, S., Ilany, A., White, B. J., Sanderson, M. W. \& Lanzas, C. Spatialtemporal dynamics of high-resolution animal networks: what can we learn from domestic animals. Plos One 10, e0129253 (2015).

6 Opsahl, T. \& Panzarasa, P. Clustering in weighted networks. Social Networks 31, 155-163 (2009).

7 Euler, L. Solutio problematis ad geometriam situs pertinentis. Commentarii Academiae Scientiarum Petropolitanae, 128-140 (1741).

8 Clauset, A., Newman, M. E. \& Moore, C. Finding community structure in very large networks. Physical Review E 70, 066111 (2004).

9 Kron, G. Tensors for Circuits. (Dover Publications, 1959).

10 Haggett, P. \& Chorley, R. J. Network Analysis in Geography. Vol. 1 (Hodder Education, 1969).

11 Freeman, L. The development of social network analysis. A Study in the Sociology of Science 1, 687 (2004).

12 Wasserman, S. \& Galaskiewicz, J. Advances in Social Network Analysis: Research in the Social and Behavioral Sciences. Vol. 171 (Sage Publications, 1994).

13 Malm, A. \& Bichler, G. Networks of collaborating criminals: assessing the structural vulnerability of drug markets. Journal of Research in Crime and Delinquency 48, 271-297 (2011).

14 Brandes, U. A faster algorithm for betweenness centrality. Journal of Mathematical Sociology 25, 163-177 (2001).

15 Freeman, L. C. Centrality in social networks conceptual clarification. Social Networks 1, 215-239 (1979).

$16 \mathrm{Li}, \mathrm{X}$. et al. Global and local persistence of influenza A (H5N1) virus. Emerging Infectious Disease 20, 1287-1295 (2014).

17 Macker, J. P. An improved local bridging centrality model for distributed network analytics. 2016 IEEE Military Communications Conference 1, 600605 (2016).

18 Tian, H. et al. Avian influenza H5N1 viral and bird migration networks in Asia. Proceedings of the National Academy of Sciences 112, 172-177 (2015).

19 Fath, B. D., Scharler, U. M., Ulanowicz, R. E. \& Hannon, B. Ecological network analysis: network construction. Ecological Modelling 208, 49-55 (2007).

20 Morales-Castilla, I., Matias, M. G., Gravel, D. \& Araújo, M. B. Inferring biotic interactions from proxies. Trends in Ecology \& Evolution 30, 347-356 (2015).

21 Kondoh, M. Foraging adaptation and the relationship between food-web complexity and stability. Science 299, 1388-1391 (2003).

22 Otto, S. B., Rall, B. C. \& Brose, U. Allometric degree distributions facilitate food-web stability. Nature 450, 1226-1229 (2007).

23 van Altena, C., Hemerik, L. \& de Ruiter, P. C. Food web stability and weighted connectance: the complexity-stability debate revisited. Theoretical Ecology 9, 49-58 (2016). 
24 Jacoby, D. M. \& Freeman, R. Emerging network-based tools in movement ecology. Trends in Ecology \& Evolution 31, 301-314 (2016).

25 Firth, J. A. Considering complexity: animal social networks and behavioural contagions. Trends in Ecology \& Evolution 35, 100-104 (2020).

26 Snijders, L. et al. Social networking in territorial great tits: slow explorers have the least central social network positions. Animal Behaviour 98, 95-102 (2014).

27 de Silva, S., Ranjeewa, A. D. \& Kryazhimskiy, S. The dynamics of social networks among female Asian elephants. BMC Ecology 11, 1-16 (2011).

28 Aplin, L. M. et al. Experimentally induced innovations lead to persistent culture via conformity in wild birds. Nature 518, 538 (2015).

29 Firth, J. A. \& Sheldon, B. C. Social carry-over effects underpin transseasonally linked structure in a wild bird population. Ecology Letters 19, 1324-1332 (2016).

30 Firth, J. A. et al. Wild birds respond to flockmate loss by increasing their social network associations to others. Proceedings of the Royal Society B: Biological Sciences 284, 20170299 (2017).

31 Grange, Z. L., Van Andel, M., French, N. P. \& Gartrell, B. D. Network analysis of translocated Takahe populations to identify disease surveillance targets. Conservation Biology 28, 518-528 (2014).

32 Calder, J. L., Cumming, G. S., Maciejewski, K. \& Oschadleus, H. D. Urban land use does not limit weaver bird movements between wetlands in Cape Town, South Africa. Biological Conservation 187, 230-239 (2015).

33 Gil-Tena, A., Brotons, L., Fortin, M. J., Burel, F. \& Saura, S. Assessing the role of landscape connectivity in recent woodpecker range expansion in Mediterranean Europe: forest management implications. European Journal of Forest Research 132, 181-194 (2013).

34 Downs, J. A. \& Horner, M. W. Spatially modelling pathways of migratory birds for nature reserve site selection. International Journal of Geographical Information Science 22, 687-702 (2008).

35 Studds, C. E. et al. Rapid population decline in migratory shorebirds relying on Yellow Sea tidal mudflats as stopover sites. Nature Communications $\mathbf{8}$, 14895 (2017).

36 Newton, I. The Migration Ecology of Birds. (Academic Press, 2010).

37 Shimazaki, H. et al. Network analysis of potential migration routes for Oriental White Storks (Ciconia boyciana). Ecological Research 19, 683-698 (2004).

38 Fretwell, S. D. Populations in a Seasonal Environment. (Princeton University Press, 1972).

39 Lameris, T. K. et al. Arctic geese tune migration to a warming climate but still suffer from a phenological mismatch. Current Biology 28, 2467-2473 (2018).

40 Lameris, T. K. et al. Potential for an Arctic-breeding migratory bird to adjust spring migration phenology to Arctic amplification. Global Change Biology 23, 4058-4067 (2017).

41 Webster, M. S., Marra, P. P., Haig, S. M., Bensch, S. \& Holmes, R. T. Links between worlds: unraveling migratory connectivity. Trends in Ecology \& Evolution 17, 76-83 (2002).

42 Patchett, R., Finch, T. \& Cresswell, W. Population consequences of migratory variability differ between flyways. Current Biology 28, 340-341 (2018).

43 Standish, R. K. Complexity of networks. Recent Advances in Artificial Life 3, 253-263 (2005). 
44 Merken, R., Deboelpaep, E., Teunen, J., Saura, S. \& Koedam, N. Wetland suitability and connectivity for trans-Saharan migratory waterbirds. Plos One 10, e0135445 (2015).

45 Bellisario, B., Cerfolli, F. \& Nascetti, G. Climate effects on the distribution of wetland habitats and connectivity in networks of migratory waterbirds. Acta Oecologica 58, 5-11 (2014).

46 Buhnerkempe, M. G. et al. Identification of migratory bird flyways in North America using community detection on biological networks. Ecological Applications 26, 740-751 (2016).

47 Lincoln, F. C. Waterfowl Flyways of North America. Information Systems Division, National Agricultural Library (1935).

48 Brooks, T. M. et al. Habitat loss and extinction in the hotspots of biodiversity. Conservation Biology 16, 909-923 (2002).

49 Rosenberg, K. V. et al. Decline of the North American avifauna. Science 366, 120-124 (2019).

50 Fox, A. et al. Effects of agricultural change on abundance, fitness components and distribution of two arctic-nesting goose populations. Global Change Biology 11, 881-893 (2005).

51 Yu, H. et al. Are declining populations of wild geese in China 'prisoners' of their natural habitats? Current Biology 27, 376-377 (2017).

52 Tombre, I. M., Oudman, T., Shimmings, P., Griffin, L. \& Prop, J. Northward range expansion in spring-staging barnacle geese is a response to climate change and population growth, mediated by individual experience. Global Change Biology 25, 3680-3693 (2019).

53 Roberts, C. P., Allen, C. R., Angeler, D. G. \& Twidwell, D. Shifting avian spatial regimes in a changing climate. Nature Climate Change 9, 562-566 (2019).

54 Huntley, B., Collingham, Y. C., Willis, S. G. \& Green, R. E. Potential impacts of climatic change on European breeding birds. Plos One 3, e1439 (2008).

55 Syroechkovskiy, E. E. in Waterbirds around the world 649-662 (2006).

56 Gilroy, J. J., Gill, J. A., Butchart, S. H. M., Jones, V. R. \& Franco, A. M. A. Migratory diversity predicts population declines in birds. Ecology Letters 19, 308-317 (2016).

57 Saura, S., Bodin, Ö. \& Fortin, M. J. Stepping stones are crucial for species' long-distance dispersal and range expansion through habitat networks. Journal of Applied Ecology 51, 171-182 (2014).

58 Burger, J. \& Gochfeld, M. Marine birds as sentinels of environmental pollution. EcoHealth 1, 263-274 (2004).

59 Xu, Y., Gong, P., Wielstra, B. \& Si, Y. Southward autumn migration of waterfowl facilitates cross-continental transmission of the highly pathogenic avian influenza H5N1 virus. Scientific Reports 6, 30262 (2016).

60 Verhagen, J. H., Herfst, S. \& Fouchier, R. A. How a virus travels the world. Science 347, 616-617 (2015).

61 Reneerkens, J. et al. Low fitness at low latitudes: wintering in the tropics increases migratory delays and mortality rates in an Arctic breeding shorebird. Journal of Animal Ecology 89, 691-703 (2020).

62 Hedenström, A. \& Alerstam, T. Optimum fuel loads in migratory birds: distinguishing between time and energy minimization. Journal of Theoretical Biology 189, 227-234 (1997).

63 Groen, T. A. \& Prins, H. H. T. in Bird migration across the Himalayas: wetland functioning amidst mountains and glaciers 254-268 (Cambridge University Press, 2017). 
Prins, H. H., Jansen, R. J. \& Vélez, V. M. in Bird Migration Across the Himalayas: Wetland Functioning amidst Mountains and Glaciers 269-282 (Cambridge University Press, 2017).

65 Clausen, P., Green, M. \& Alerstam, T. Energy limitations for spring migration and breeding: the case of brent geese Branta bernicla tracked by satellite telemetry to Svalbard and Greenland. Oikos 103, 426-445 (2003).

66 Drent, R. \& Daan, S. The prudent parent: energetic adjustments in avian breeding. Ardea 68, 225-252 (1980).

67 Arzel, C., Elmberg, J. \& Guillemain, M. Ecology of spring-migrating Anatidae: a review. J Ornithol 147, 167-184 (2006).

68 Grishchenko, M. et al. Land use change and the migration geography of Greater White-fronted geese in European Russia. Ecosphere 10, e02754 (2019).

69 Hirzel, A. H. \& Le Lay, G. Habitat suitability modelling and niche theory. Journal of Applied Ecology 45, 1372-1381 (2008).

70 Saura, S. \& Pascual-Hortal, L. A new habitat availability index to integrate connectivity in landscape conservation planning: comparison with existing indices and application to a case study. Landscape and Urban Planning 83, 91-103 (2007).

71 Russell, R. W., Carpenter, F. L., Hixon, M. A. \& Paton, D. C. The impact of variation in stopover habitat quality on migrant Rufous Hummingbirds. Conservation Biology 8, 483-490 (1994).

72 Henkel, J. R., Sigel, B. J. \& Taylor, C. M. Large-scale impacts of the Deepwater Horizon oil spill: Can local disturbance affect distant ecosystems through migratory shorebirds? BioScience 62, 676-685 (2012).

73 Iwamura, T. et al. Migratory connectivity magnifies the consequences of habitat loss from sea-level rise for shorebird populations. Proceedings of the Royal Society of London B: Biological Sciences 280, 20130325 (2013).

74 Sanderson, F. J., Donald, P. F., Pain, D. J., Burfield, I. J. \& Van Bommel, F. P. Long-term population declines in Afro-Palearctic migrant birds. Biological Conservation 131, 93-105 (2006).

75 Millennium Ecosystem Assessment. Ecosystems and Human Well-being: Wetlands and Water. (World Resources Institute, 2005).

76 Silva, J. P., Phillips, L. \& Jones, W. Life and Europe's Wetlands: Restoring a Vital Ecosystem. (European Commission Environment Directorategenera, 2007).

77 Niu, Z. et al. Mapping wetland changes in China between 1978 and 2008. Chinese Science Bulletin 57, 2813-2823 (2012).

78 Wang, $Z$. et al. Changes of land use and of ecosystem service values in Sanjiang Plain, Northeast China. Environmental Monitoring and Assessment 112, 69-91 (2006).

79 Mora, J. W., Mager III, J. N. \& Spieles, D. J. Habitat and landscape suitability as indicators of bird abundance in created and restored wetlands. ISRN Ecology 2011, 297684 (2011).

80 Guadagnin, D. L. \& Maltchik, L. Habitat and landscape factors associated with neotropical waterbird occurrence and richness in wetland fragments. Biodiversity and Conservation 16, 1231-1244 (2007).

81 Zhang, W., li, X., Yu, L. \& Si, Y. Multi-scale habitat selection by two declining East Asian waterfowl species at their core spring stopover area. Ecological Indicators 87, 127-135 (2018).

$82 \mathrm{Xu}, \mathrm{C}$. et al. Can local landscape attributes explain species richness patterns at macroecological scales? Global Ecology and Biogeography 23, 436-445 (2014). 
83 Van Eerden, M. R., Drent, R. H., Stahl, J. \& Bakker, J. P. Connecting seas: western Palaearctic continental flyway for water birds in the perspective of changing land use and climate. Global Change Biology 11, 894-908 (2005).

84 Tian, B., Zhou, Y., Zhang, L. \& Yuan, L. Analyzing the habitat suitability for migratory birds at the Chongming Dongtan Nature Reserve in Shanghai, China. Estuarine Coastal and Shelf Science 80, 296-302 (2008).

85 Dong, Z., Wang, Z. \& Yang, X. Changes in suitable waterbirds' habitats from 1990 to 2010 in the Bielahong Watershed of Northeast China. Journal Indian Society of Remote Sensing 43, 599-607 (2015).

86 Cao, L., Barter, M. \& Lei, G. New Anatidae population estimates for eastern China: Implications for current flyway estimates. Biological Conservation 141 (2008).

87 Cao, L., Zhang, Y., Barter, M. \& Lei, G. Anatidae in eastern China during the non-breeding season: geographical distributions and protection status. Biological Conservation 143, 650-659 (2010).

88 de Boer, W. F. et al. Comparing the community composition of European and Eastern Chinese waterbirds and the influence of human factors on the China waterbird community. AMBIO 40, 68-77 (2011).

$89 \mathrm{Si}$, Y. et al. Spring migration patterns, habitat use, and stopover site protection status for two declining waterfowl species wintering in China as revealed by satellite tracking. Ecology and Evolution 8, 6280-6289 (2018).

90 Batbayar, N. et al. Migration strategies of Swan Geese Anser cygnoides from northeast Mongolia. Wildfowl 61, 90-109 (2013).

91 Takekawa, J. Y. et al. Migration of waterfowl in the East Asian Flyway and spatial relationship to HPAI H5N1 outbreaks. Avian Diseases 54, 466-476 (2010).

92 Morrison, C. A., Robinson, R. A., Clark, J. A., Risely, K. \& Gill, J. A. Recent population declines in Afro-Palaearctic migratory birds: the influence of breeding and non-breeding seasons. Diversity and Distributions 19, 10511058 (2013).

93 Weber, T. P., Houston, A. I. \& Ens, B. J. Consequences of habitat loss at migratory stopover sites: a theoretical investigation. Journal of Avian Biology 30, 416-426 (1999).

94 Runge, C. A., Martin, T. G., Possingham, H. P., Willis, S. G. \& Fuller, R. A. Conserving mobile species. Frontiers in Ecology and the Environment 12, 395-402 (2014).

95 BirdLife International and NatureServe. Bird Species Distribution Maps of the World Version 5.0. Available online from http://www.birdlife.org. Accessed on 02-11-2016.

96 Sullivan, B. L. et al. The eBird enterprise: an integrated approach to development and application of citizen science. Biological Conservation 169, 31-40 (2014).

97 Cornell Lab of Ornithology. eBird Basic Dataset Vesion EBD_relNov-2016. Available online from https://ebird.org/science. Accessed on 06-01-2017.

98 Lehner, B. \& Döll, P. Development and validation of a global database of lakes, reservoirs and wetlands. Journal of Hydrology 296, 1-22 (2004).

99 de Ferranti, J. Jonathan de Ferranti's Digital Elevation Data Site. Available online from http://www.viewfinderpanoramas.org. Accessed on 07-03-2017.

100 European Space Agency. CCI Land Cover - 300 m Annual Global Land Cover Time Series from 1992 to 2015. Available at: https://www.esa-landcovercci.org. Accessed on 28-07-2017.

101 Davis, J. B. et al. Habitat and resource use by waterfowl in the northern hemisphere in autumn and winter. Wildfowl 2014, 17-69 (2014). 
102 Beatty, W. S. et al. Landscape effects on mallard habitat selection at multiple spatial scales during the non-breeding period. Landscape Ecology 29, 9891000 (2014).

103 Johnson, W. P., Schmidt, P. M. \& Taylor, D. P. Foraging flight distances of wintering ducks and geese: a review. Avian Conservation and Ecology 9 (2014).

104 Burnham, K. P. \& Anderson, D. R. Model Selection and Multimodel Inference: a Practical Information-Theoretic Approach. (Springer Science \& Business Media, 2003).

105 Olson, D. L. \& Delen, D. Advanced Data Mining Techniques. (Springer Science \& Business Media, 2008).

106 Bates, D., Mächler, M., Bolker, B. \& Walker, S. Fitting linear mixed-effects models using lme4. arXiv:1406.5823 (2014).

107 Horn, D. J. et al. Landscape composition, patch size, and distance to edges: Interactions affecting duck reproductive success. Ecological Applications 15, 1367-1376 (2005).

108 Zhang, Y., Jia, Q., Prins, H. H., Cao, L. \& de Boer, W. F. Individual-area relationship best explains goose species density in wetlands. PLoS One 10, e0124972 (2015).

109 Ackerman, J. T. et al. Spatial use by wintering greater white-fronted geese relative to a decade of habitat change in California's Central Valley. The Journal of Wildlife Management 70, 965-976 (2006).

$110 \mathrm{Si}$, Y. et al. Distribution of Barnacle Geese Branta leucopsis in relation to food resources, distance to roosts, and the location of refuges. Ardea 99, 217-226 (2011).

111 McGill, B. J. Matters of scale. Science 328, 575-576 (2010).

112 Gustafson, E. J. \& Parker, G. R. Relationships between landcover proportion and indices of landscape spatial pattern. Landscape Ecology 7, 101-110 (1992).

113 McGarigal, K. \& Marks, B. J. FRAGSTATS: Spatial Pattern Analysis Program for Quantifying Landscape Structure. (US Department of Agriculture, Forest Service, Pacific Northwest Research Station, 1995).

114 Sawyer, H., Kauffman, M. J., Nielson, R. M. \& Horne, J. S. Identifying and prioritizing ungulate migration routes for landscape-level conservation. Ecological Applications 19, 2016-2025 (2009).

115 Berger, J., Young, J. K. \& Berger, K. M. Protecting migration corridors: challenges and optimism for Mongolian saiga. PLos Biology 6, e165 (2008).

116 North American Bird Conservation Initiative. The State of Canada's Birds. (Environment Canada, 2012).

117 Batbayar, N. Breeding and Migration Ecology of Bar-headed Goose Anser indicus and Swan Goose Anser cygnoides in Asia (University of Oklahoma, 2013).

118 Seto, K. C. \& Fragkias, M. Quantifying spatiotemporal patterns of urban land-use change in four cities of China with time series landscape metrics. Landscape Ecology 20, 871-888 (2005).

119 Fox, A. D., Elmberg, J., Tombre, I. M. \& Hessel, R. Agriculture and herbivorous waterfowl: a review of the scientific basis for improved management. Biological Reviews 92, 854-877 (2016).

120 Jia, Q., Wang, X., Zhang, Y., Cao, L. \& Fox, A. D. Drivers of waterbird communities and their declines on Yangtze River floodplain lakes. Biological Conservation 218, 240-246 (2018).

121 Liu, J. \& Diamond, J. China's environment in a globalizing world. Nature 435, 1179 (2005). 
122 Nicholls, R. J. Coastal flooding and wetland loss in the 21 st century: changes under the SRES climate and socio-economic scenarios. Global Environmental Change 14, 69-86 (2004).

123 Grishchenko, M. \& Prins, H. H. Abandoned field succession in Russia and its potential effect on Corncrake Crex crex habitats. Die Vogelwelt 136, 175184 (2016).

$124 \mathrm{Li}, \mathrm{Z}$. et al. Land use/cover change and regional climate change in an arid grassland ecosystem of Inner Mongolia, China. Ecological Modelling 353, 8694 (2017).

125 Li, J., Lai, X., Liu, H., Yang, D. \& Zhang, G. Emergy evaluation of three rice wetland farming systems in the Taihu Lake catchment of China. Wetlands 38, 1-12 (2017).

126 Li, X., Si, Y., Ji, L. \& Gong, P. Dynamic response of East Asian greater whitefronted geese to changes of environment during migration: Use of multitemporal species distribution model. Ecological Modelling 360, 70-79 (2017).

127 Aharon-Rotman, Y. et al. Water level affects availability of optimal feeding habitats for threatened migratory waterbirds. Ecology and Evolution 7, 10440-10450 (2017).

128 MaMing, R., Zhang, T., Blank, D., Ding, P. \& Zhao, X. Geese and ducks killed by poison and analysis of poaching cases in China. Goose Bulletin 15, 2-11 (2012).

129 An, S. et al. China's natural wetlands: past problems, current status, and future challenges. AMBIO 36, 335-342 (2007).

$130 \mathrm{Li}, \mathrm{Z}$. et al. Ecological restoration and its effects on a regional climate: the source region of the Yellow River, China. Environmental Science \& Technology 49, 5897-5904 (2015).

131 Purvis, A., Gittleman, J. L., Cowlishaw, G. \& Mace, G. M. Predicting extinction risk in declining species. Proceedings of the Royal Society of London B: Biological Sciences 267, 1947-1952 (2000).

132 MacArthur, R. \& Wilson, E. The Theory Of Island Biogeography. (Princeton University Press, 1967).

133 Sutherland, W. J. et al. A horizon scanning assessment of current and potential future threats to migratory shorebirds. Ibis 154, 663-679 (2012).

134 Wetlandsa International. Waterbird Population Estimates (Wetlands International, 2017).

135 Rogers, D. I. et al. Red Knots (Calidris canutus piersmai and C. c. rogersi) depend on a small threatened staging area in Bohai Bay, China. Emu-Austral Ornithology 110, 307-315 (2010).

136 Si, Y., Xin, Q., Prins, H. H. T., de Boer, W. F. \& Gong, P. Improving the quantification of waterfowl migration with remote sensing and bird tracking. Science Bulletin 60, 1984-1993 (2015).

$137 \mathrm{Xu}, \mathrm{W}$. et al. Reassessing the conservation status of the giant panda using remote sensing. Nature Ecology \& Evolution 1, 1635 (2017).

138 Cushman, S. A. Effects of habitat loss and fragmentation on amphibians: a review and prospectus. Biological Conservation 128, 231-240 (2006).

139 Taylor, P. D., Fahrig, L., Henein, K. \& Merriam, G. C. F. Connectivity is a vital element of landscape structure. Oikos 68, 571-573 (1993).

140 Saura, S. \& Rubio, L. A common currency for the different ways in which patches and links can contribute to habitat availability and connectivity in the landscape. Ecography 33, 523-537 (2010).

141 Bélisle, M. Measuring landscape connectivity: the challenge of behavioral landscape ecology. Ecology 86, 1988-1995 (2005). 
142 Leito, A. et al. The potential impacts of changes in ecological networks, land use and climate on the Eurasian crane population in Estonia. Landscape Ecology 30, 887-904 (2015).

143 Murray, K. A., Verde Arregoitia, L. D., Davidson, A., Di Marco, M. \& Di Fonzo, M. M. Threat to the point: improving the value of comparative extinction risk analysis for conservation action. Global Change Biology 20, 483-494 (2014).

144 Jiguet, F., Gadot, A. S., Julliard, R., Newson, S. E. \& Couvet, D. Climate envelope, life history traits and the resilience of birds facing global change. Global Change Biology 13 (2007).

145 Zhang, Y., Jia, Q., Prins, H. H. T., Cao, L. \& de Boer, W. F. Effect of conservation efforts and ecological variables on waterbird population sizes in wetlands of the Yangtze River. Scientific Reports 5, 17136 (2015).

146 Keitt, T. H., Urban, D. L. \& Milne, B. T. Detecting critical scales in fragmented landscapes. Conservation Ecology 1, 4 (1997).

$147 \mathrm{Xu}$, Y. et al. Species-dependent effects of habitat degradation in relation to seasonal distribution of migratory waterfowl in the East Asian-Australasian Flyway. Landscape Ecology 34, 243-257 (2019).

148 Saura, S. \& Torne, J. Conefor Sensinode 2.2: a software package for quantifying the importance of habitat patches for landscape connectivity. Environmental Modelling \& Software 24, 135-139 (2009).

149 Myhrvold, N. P. et al. An amniote life-history database to perform comparative analyses with birds, mammals, and reptiles. Ecology 96, 31093109 (2015).

150 BirdLife International. The IUCN Red List of Threatened Species. (International Union for Conservation of Nature, 2016).

151 Pearse, W. D. et al. pez: phylogenetics for the environmental sciences. Bioinformatics 31, 2888-2890 (2015).

152 Ives, A. R. \& Helmus, M. R. Generalized linear mixed models for phylogenetic analyses of community structure. Ecological Monographs 81, 511-525 (2011).

153 Ericson, P. G. et al. Diversification of Neoaves: integration of molecular sequence data and fossils. Biology Letters 2, 543-547 (2006).

154 Jetz, W., Thomas, G., Joy, J., Hartmann, K. \& Mooers, A. The global diversity of birds in space and time. Nature 491, 444-448 (2012).

155 Schmaljohann, H. \& Both, C. The limits of modifying migration speed to adjust to climate change. Nature Climate Change 7, 573-576 (2017).

156 Goymann, W., Spina, F., Ferri, A. \& Fusani, L. Body fat influences departure from stopover sites in migratory birds: evidence from whole-island telemetry. Biology Letters 6, 478-481 (2010).

157 Norris, D. R., Marra, P. P., Kyser, T. K., Sherry, T. W. \& Ratcliffe, L. M. Tropical winter habitat limits reproductive success on the temperate breeding grounds in a migratory bird. Proceedings of the Royal Society of London B: Biological Sciences 271, 59-64 (2004).

158 Norris, D. R. \& Taylor, C. M. Predicting the consequences of carry-over effects for migratory populations. Biology letters 2, 148-151 (2006).

159 Crooks, K. R. \& Sanjayan, M. Connectivity Conservation. Vol. 14 (Cambridge University Press, 2006).

160 Schmaljohann, H., Liechti, F. \& Bruderer, B. Songbird migration across the Sahara: the non-stop hypothesis rejected! Proceedings of the Royal Society of London B: Biological Sciences 274, 735-739 (2007).

161 Prins, H. H. \& Namgail, T. Bird Migration across the Himalayas: Wetland Functioning amidst Mountains and Glaciers. (Cambridge University Press, 2017). 
$162 \mathrm{Xu}, \mathrm{C}$. et al. Can local landscape attributes explain species richness patterns at macroecological scales? Global Ecology and Biogeography 23, 436-445 (2014).

163 Hewson, C. M., Thorup, K., Pearce-Higgins, J. W. \& Atkinson, P. W. Population decline is linked to migration route in the Common Cuckoo. Nature Communications 7, 12296 (2016).

164 Verkuil, Y. I. et al. Losing a staging area: Eastward redistribution of AfroEurasian ruffs is associated with deteriorating fuelling conditions along the western flyway. Biological Conservation 149, 51-59 (2012).

165 Zou, Y. A. et al. Migratory waterbirds response to coastal habitat changes: conservation implications from long-term detection in the Chongming Dongtan Wetlands, China. Estuaries and Coasts 39, 273-286 (2016).

166 Kölzsch, A. et al. Towards a new understanding of migration timing: slower spring than autumn migration in geese reflects different decision rules for stopover use and departure. Oikos 125, 1496-1507 (2016).

167 Beekman, J. H., Nolet, B. A. \& Klaassen, M. Skipping swans: fuelling rates and wind conditions determine differential use of migratory stopover sites of Bewick's Swans Cygnus bewickii. Ardea 90, 437-460 (2002).

168 Miller, M. R. et al. Flight speeds of northern pintails during migration determined using satellite telemetry. The Wilson Bulletin 117, 364-374 (2005).

$169 \mathrm{Xu}, \mathrm{Y}$. et al. Loss of functional connectivity in migration networks induces population decline in migratory birds. Ecological Applications 29, e01960 (2019).

$170 \mathrm{Xu}, \mathrm{Y}$. et al. A network approach to prioritize conservation efforts for migratory birds. Conservation Biology 34, 416-426 (2019).

171 Mathers, R. \& Montgomery, W. Quality of food consumed by over wintering Pale-Bellied Brent Geese Branta bernicla hrota and Wigeon Anas penelope. Biology and Environment 978, 81-89 (1997).

172 Barbaree, B. A., Reiter, M. E., Hickey, C. M. \& Page, G. W. Day and night habitat associations of wintering Dunlin (Calidris alpina) within an agriculture-wetland mosaic. Waterbirds 38, 40-46 (2015).

173 Lonsdorf, E. V. et al. A generalizable energetics-based model of avian migration to facilitate continental-scale waterbird conservation. Ecological Applications 26 (2016).

174 Tucker, M. A. et al. Large birds travel farther in homogeneous environments. Global Ecology and Biogeography 28, 576-587 (2019).

175 Riddington, R., Hassall, M., Lane, S., Turner, P. \& Walters, R. The impact of disturbance on the behaviour and energy budgets of Brent Geese Branta $b$. bernicla. Bird Study 43, 269-279 (1996).

176 Gueguen, L. Segmentation by maximal predictive partitioning according to composition biases. International Conference on Biology, Informatics, and Mathematics 32-44 (2000).

177 Calenge, C. Analysis of Animal Movements in $r$ : the adehabitatLT Package. (R Foundation for Statistical Computing, Vienna, 2011).

178 Stephens, P. A., Houston, A. I., Harding, K. C., Boyd, I. L. \& McNamara, J. M. Capital and income breeding: the role of food supply. Ecology 95, 882896 (2014).

179 Taft, O. W., Sanzenbacher, P. M. \& Haig, S. M. Movements of wintering Dunlin Calidris alpina and changing habitat availability in an agricultural wetland landscape. Ibis 150, 541-549 (2008).

180 Aben, J. et al. Effects of matrix composition and configuration on forest bird movements in a fragmented Afromontane biodiversity hot spot. Animal Conservation 15, 658-668 (2012). 
181 De Knegt, H. J. et al. The spatial scaling of habitat selection by African elephants. Journal of Animal Ecology 80, 270-281 (2011).

182 Kranstauber, B., Kays, R., LaPoint, S. D., Wikelski, M. \& Safi, K. A dynamic Brownian bridge movement model to estimate utilization distributions for heterogeneous animal movement. Journal of Animal Ecology 81, 738-746 (2012).

183 Arzel, C. et al. Experimental functional response and inter-individual variation in foraging rate of teal (Anas crecca). Behavioural Processes 75, 6671 (2007).

184 Madsen, J. Experimental refuges for migratory waterfowl in Danish wetlands. I. Baseline assessment of the disturbance effects of recreational activities. Journal of Applied Ecology 35, 386-397 (1998).

185 Fox, A. D. et al. Current estimates of goose population sizes in western Europe, a gap analysis and assessment of trends. Ornis Svecica 20, 115-127 (2010).

186 Eythórsson, E., Tombre, I. M. \& Madsen, J. Goose management schemes to resolve conflicts with agriculture: Theory, practice and effects. AMBIO 46, 231-240 (2017).

187 Cui, B., He, Q., Gu, B., Bai, J. \& Liu, X. China's Coastal Wetlands: Understanding Environmental Changes and Human Impacts for Management and Conservation. Wetlands 36, 1-9 (2016).

$188 \mathrm{Xu}, \mathrm{Y}$. et al. Species-dependent effects of habitat degradation in relation to seasonal distribution of migratory waterfowl in the East Asian-Australasian Flyway. Landscape Ecology 34, 243-257 (2019).

189 de Weerd, N. et al. Deriving animal behaviour from high-frequency GPS: tracking cows in open and forested habitat. Plos One 10, e0129030 (2015).

190 Dhanjal-Adams, K. L. et al. Setting conservation priorities for migratory networks under uncertainty. Conservation Biology 31, 646-656 (2017).

191 Mehlman, D. W. et al. Conserving stopover sites for forest-dwelling migratory landbirds. The Auk 122, 1281-1290 (2005).

192 Dunn, E. H. A cross-Canada comparison of mass change in birds during migration stopover. The Wilson Journal of Ornithology 114, 368-380 (2002).

193 Bayly, N. J., Gómez, C., Hobson, K. A., González, A. M. \& Rosenberg, K. V. Fall migration of the Veery (Catharus fuscescens) in northern Colombia: determining the energetic importance of a stopover site. The Auk 129, 449459 (2012).

194 Bibby, C. J. Avian Migration 407-420. (Springer, 2003).

195 Bieri, J. A. et al. A guide to calculating habitat-quality metrics to inform conservation of highly mobile species. Natural Resource Modeling 31, e12156 (2018).

196 Nicol, S. et al. A management-oriented framework for selecting metrics used to assess habitat-and path-specific quality in spatially structured populations. Ecological Indicators 69, 792-802 (2016).

197 UN Environment. Convention on migratory species. UNEP/CMS/Resolution $12.7 \&$ UNEP/CMS/Resolution 12.26 (United Nations Environment Programme, 2017).

198 Baker, A. J. et al. Rapid population decline in red knots: fitness consequences of decreased refuelling rates and late arrival in Delaware Bay. Proceedings of the Royal Society of London. Series B: Biological Sciences 271, 875-882 (2004).

199 Hostetler, J. A., Sillett, T. S. \& Marra, P. P. Full-annual-cycle population models for migratory birds. Auk 132, 433-449 (2015).

200 Sample, C. et al. A general modeling framework for describing spatially structured population dynamics. Ecology and Evolution 8, 493-508 (2018). 
201 Jensen, R. A., Wisz, M. S. \& Madsen, J. Prioritizing refuge sites for migratory geese to alleviate conflicts with agriculture. Biological Conservation 141, 1806-1818 (2008).

202 Nicol, S., Fuller, R. A., Iwamura, T. \& Chadès, I. Adapting environmental management to uncertain but inevitable change. Proceedings of the Royal Society B: Biological Sciences 282, 20142984 (2015).

203 Iwamura, T., Fuller, R. A. \& Possingham, H. P. Optimal management of a multispecies shorebird flyway under sea-level rise. Conservation Biology 28, 1710-1720 (2014).

204 Newman, S. H. et al. Eco-virological approach for assessing the role of wild birds in the spread of avian influenza H5N1 along the Central Asian Flyway. Plos One 7, e30636 (2012).

205 Drent, R. H., Fox, A. D. \& Stahl, J. Travelling to breed. Journal of Ornithology 147, 122-134 (2006).

206 McRae, B. H., Dickson, B. G., Keitt, T. H. \& Shah, V. B. Using circuit theory to model connectivity in ecology, evolution, and conservation. Ecology $\mathbf{8 9}$, 2712-2724 (2008).

207 Newman, M. E. A measure of betweenness centrality based on random walks. Social Networks 27, 39-54 (2005).

208 Dokter, A. M. et al. Seasonal abundance and survival of North America's migratory avifauna determined by weather radar. Nature Ecology \& Evolution 2, 1603 (2018).

209 Dhanjal-Adams, K. L. et al. Spatiotemporal group dynamics in a longdistance migratory bird. Current Biology (2018).

210 Van Mieghem, P., Devriendt, K. \& Cetinay, H. Pseudoinverse of the Laplacian and best spreader node in a network. Physical Review E 96, 032311 (2017).

211 Gong, P. et al. China's wetland change (1990-2000) determined by remote sensing. Science China-Earth Sciences 53, 1036-1042 (2010).

212 Wetlands International. Resolution XI.8, Annex 1: Ramsar site information sheet (RIS) - 2012 revision (Wetlands International, 2012).

213 Wasserman, S. \& Faust, K. Social Network Analysis: Methods and Applications. Vol. 8 (Cambridge University Press, 1994).

214 Dijkstra, E. W. A note on two problems in connexion with graphs. Numerische Mathematik 1, 269-271 (1959).

215 O'sullivan, D. \& Unwin, D. Geographic Information Analysis. (John Wiley \& Sons, 2014).

216 Olsen, B. et al. Global patterns of influenza A virus in wild birds. science 312, 384-388 (2006).

217 Chen, H. et al. Avian flu: H5N1 virus outbreak in migratory waterfowl. Nature 436, 191 (2005).

218 Takekawa, J. Y. et al. Victims and vectors: highly pathogenic avian influenza H5N 1 and the ecology of wild birds. Avian Biology Research 3, 51-73 (2010).

219 Kilpatrick, A. M. et al. Predicting the global spread of H5N1 avian influenza. Proceedings of the National Academy of Sciences 103, 19368-19373 (2006).

$220 \mathrm{Ge}, \mathrm{Z}$. M. et al. Effects of Vegetative Cover Changes on the Carrying Capacity of Migratory Shorebirds in a Newly Formed Wetland, Yangtze River Estuary, China. Zoological Studies 48, 769-779 (2009).

221 Lycett, S. J. et al. Role for migratory wild birds in the global spread of avian influenza H5N8. Science 354, 213-217 (2016).

222 Si, Y. et al. Spatio-temporal dynamics of global H5N1 outbreaks match bird migration patterns. Geospatial Health 4, 65-78 (2009).

223 Liang, L. et al. Combining spatial-temporal and phylogenetic analysis approaches for improved understanding on global H5N 1 transmission. Plos One 5 (2010). 
224 Ramanathan, M., Zhang, A., Cho, Y \& Hwang, W. Bridging centrality: identifying bridging nodes in scale-free networks. [Available online at https://pdfs.semanticscholar.org/ac78/566bf6b3571b25921e8598733c881d 9c35e9.pdf] (2006).

225 Teitelbaum, C. S. et al. Experience drives innovation of new migration patterns of whooping cranes in response to global change. Nature Communications 7 (2016).

226 BirdLife International. Climate change may force European species northwards. (BirdLife International 2008).

227 Zalakevicius, M. Biophysical impacts of climate change on bird populations and migration in Lithuania. GeoJournal 57, 183-193 (2002).

228 Gunnarsson, G., Waldenström, J. \& Fransson, T. Direct and indirect effects of winter harshness on the survival of Mallards Anas platyrhynchos in northwest Europe. Ibis 154, 307-317 (2012).

229 Elmberg, J., Hessel, R., Fox, A. D. \& Dalby, L. Interpreting seasonal range shifts in migratory birds: a critical assessment of 'short-stopping'and a suggested terminology. Journal of Ornithology 155, 571-579 (2014).

230 Sauter, A., Korner-Nievergelt, F. \& Jenni, L. Evidence of climate change effects on within-winter movements of European Mallards Anas platyrhynchos. Ibis 152, 600-609 (2010).

231 Guillemain, M. et al. Effects of climate change on European ducks: what do we know and what do we need to know? Wildlife Biology 19, 404-419 (2013).

232 Csardi, G. \& Nepusz, T. The igraph software package for complex network research. InterJournal, Complex Systems 1695, 1-9 (2006).

233 R Core Team. R: A language and environment for statistical computing. (R, 2013).

234 Pons, P. \& Latapy, M. Computing communities in large networks using random walks. Journal of Graph Algorithms and Applications 10, 191-218 (2006).

235 Devlin, K. The Joy of Sets: Fundamentals of Contemporary Set Theory. (Springer Science \& Business Media, 1993).

236 Visser, M. E., Perdeck, A. C., van Balen, J. H. \& Both, C. Climate change leads to decreasing bird migration distances. Global Change Biology 15, 1859-1865 (2009).

237 Prosser, D. et al. Mapping avian influenza transmission risk at the interface of domestic poultry and wild birds. Frontiers in Public Health 1, 28 (2013).

238 Bouwstra, R. et al. Risk for low pathogenicity avian influenza virus on poultry farms, the Netherlands, 2007-2013. Emerging Infectious Diseases 23, 1510 (2017).

239 Vittecoq, M. et al. High influenza A virus infection rates in mallards bred for hunting in the Camargue, South of France. Plos One 7 (2012).

240 Begon, M. et al. A clarification of transmission terms in host-microparasite models: numbers, densities and areas. Epidemiology and Infection 129, 147153 (2002).

241 Gaidet, N. et al. Understanding the ecological drivers of avian influenza virus infection in wildfowl: a continental-scale study across Africa. Proceedings of the Royal Society B: Biological Sciences 279, 1131-1141 (2011).

242 Moran, J. M. Meteorology; the Atmosphere and the Science of Weather. (Pearson College Div, 1989).

243 Kattsov, V., Govorkova, V., Meleshko, V., Pavlova, T. \& Shkolnik, I. Climate change projections and impacts in Russian Federation and central Asia states. North Eurasia Climate Centre, Saint Petersburg, Russia.[Available online at http:// neacc. meteoinfo.ru/research/20-research] (2008). 
244 Guillemain, M. et al. Disentangling the drivers of change in Common Teal migration phenology over 50 years: land use vs. climate change effects. Journal of Ornithology 156, 647-655 (2015).

245 Van Dijk, J. G. et al. Juveniles and migrants as drivers for seasonal epizootics of avian influenza virus. Journal of Animal Ecology 83, 266-275 (2014).

246 Hinshaw, V., Webster, R., Bean, W. \& Sriram, G. The ecology of influenza viruses in ducks and analysis of influenza viruses with monoclonal antibodies. Comparative Immunology, Microbiology and Infectious Diseases 3 , 155-164 (1980).

247 Munster, V. J. et al. Spatial, temporal, and species variation in prevalence of influenza A viruses in wild migratory birds. Plos Pathogens 3, e61 (2007).

248 Berhane, Y. et al. Pre-exposing Canada geese (Branta canadensis) to a lowpathogenic $\mathrm{H} 1 \mathrm{~N} 1$ avian influenza virus protects them against H5N1 HPAI virus challenge. Journal of Wildlife Diseases 50, 84-97 (2014).

249 Pasick, J. et al. Susceptibility of Canada geese (Branta canadensis) to highly pathogenic avian influenza virus (H5N1). Emerging Infectious Diseases 13, 1821 (2007).

250 Thorup, K., Korner-Nievergelt, F., Cohen, E. B. \& Baillie, S. R. Large-scale spatial analysis of ringing and re-encounter data to infer movement patterns: A review including methodological perspectives. Methods in Ecology and Evolution 5, 1337-1350 (2014).

251 Folliot, B., Guillemain, M., Champagnon, J. \& Caizergues, A. Patterns of spatial distribution and migration phenology of common pochards Aythya ferina in the Western Palearctic: a ring-recoveries analysis. Wildlife Biology $\mathbf{1}$ (2018).

252 Yin, S. Consequences of Seasonal Migration: How Goose Relocation Strategies Influence Infection Prevalence and Pathogen Dispersal. (Wageningen University, 2018).

253 Somveille, M. et al. Movement and conformity interact to establish local behavioural traditions in animal populations. Plos Computational Biology 14, e1006647 (2018).

254 Ydenberg, R., Prins, H. \& Van Dijk, J. Post-roost gatherings of wintering barnacle geese: information centres? Ardea 71, 125-131 (1983).

255 Ydenberg, R. \& Prins, H. T. Why do birds roost communally? (1983).

256 Kurvers, R. H. et al. Social information use by barnacle geese Branta leucopsis, an experiment revisited. Ardea 102, 173-180 (2014).

257 Kurvers, R. H. et al. Personality predicts the use of social information. Ecology Letters 13, 829-837 (2010).

258 Firth, J. A., Voelkl, B., Farine, D. R. \& Sheldon, B. C. Experimental evidence that social relationships determine individual foraging behavior. Current Biology 25, 3138-3143 (2015).

259 Lima, S. L. \& Dill, L. M. Behavioral decisions made under the risk of predation: a review and prospectus. Canadian Journal of Zoology 68, 619640 (1990).

260 Voelkl, B., Firth, J. A. \& Sheldon, B. C. Nonlethal predator effects on the turn-over of wild bird flocks. Scientific Reports 6, 33476 (2016).

261 Lavers, J. L., Wilcox, C. \& Donlan, C. J. Bird demographic responses to predator removal programs. Biological Invasions 12, 3839-3859 (2010).

262 Kahlert, J. The constraint on habitat use in wing-moulting Greylag Geese Anser anser caused by anti-predator displacements. Ibis 145, 45-52 (2003).

263 Schneider, M. F. Habitat loss, fragmentation and predator impact: spatial implications for prey conservation. Journal of Applied Ecology 38, 720-735 (2001). 
264 Layman, C. A., Quattrochi, J. P., Peyer, C. M. \& Allgeier, J. E. Niche width collapse in a resilient top predator following ecosystem fragmentation. Ecology Letters 10, 937-944 (2007).

265 Byholm, P., Nikila, A., Kentta, J. \& TIaivalmak, J. P. Interactions between habitat heterogeneity and food affect reproductive output in a top predator. Journal of Animal Ecology 76, 392-401 (2007).

266 Ryall, K. L. \& Fahrig, L. Response of predators to loss and fragmentation of prey habitat: a review of theory. Ecology 87, 1086-1093 (2006).

267 Buckley, A. et al. Serological evidence of West Nile virus, Usutu virus and Sindbis virus infection of birds in the UK. Journal of General Virology 84, 2807-2817 (2003).

268 Tuyttens, F. A. M. et al. Spatial perturbation caused by a badger (Meles meles) culling operation: implications for the function of territoriality and the control of bovine tuberculosis (Mycobacterium bovis). Journal of Animal Ecology 69, 815-828 (2000).

269 Macdonald, D. Rabies in a Changing World. (Wiley Online Library, 1995).

270 Singer, A. \& Smith, G. C. Emergency rabies control in a community of two high-density hosts. BMC Veterinary Research 8, 79 (2012).

271 van Leeuwen, C. H., Van der Velde, G., van Groenendael, J. M. \& Klaassen, M. Gut travellers: internal dispersal of aquatic organisms by waterfowl. Journal of Biogeography 39, 2031-2040 (2012).

272 Charalambidou, I. \& Santamaria, L. Field evidence for the potential of waterbirds as dispersers of aquatic organisms. Wetlands 25, 252 (2005).

273 Green, A. J., Figuerola, J. \& Sánchez, M. I. Implications of waterbird ecology for the dispersal of aquatic organisms. Acta Oecologica 23, 177-189 (2002).

274 Kleyheeg, E., Treep, J., de Jager, M., Nolet, B. A. \& Soons, M. B. Seed dispersal distributions resulting from landscape-dependent daily movement behaviour of a key vector species, Anas platyrhynchos. Journal of Ecology 105, 1279-1289 (2017).

275 Kraus, R. H. S. et al. Evolution and connectivity in the world-wide migration system of the mallard: Inferences from mitochondrial DNA. BMC Genetics 12, 99 (2011).

276 Jonker, R. M. et al. Genetic consequences of breaking migratory traditions in barnacle geese Branta leucopsis. Molecular Ecology 22, 5835-5847 (2013).

277 Ottenburghs, J., van Hooft, P., van Wieren, S. E., Ydenberg, R. C. \& Prins, H. H. T. Hybridization in geese: a review. Frontiers in Zoology 13, 20 (2016).

278 Ottenburghs, J., van Hooft, P., van Wieren, S. E., Ydenberg, R. C. \& Prins, H. H. T. Birds in a bush: Toward an avian phylogenetic network. Auk 133, 577-582 (2016).

279 McNamara, J. M., Houston, A. I. \& Lima, S. L. Foraging routines of small birds in winter: a theoretical investigation. Journal of Avian Biology 25, 287302 (1994).

280 Amezaga, J., Santamaria, L. \& Green, A. J. Biotic wetland connectivitysupporting a new approach for wetland policy. Acta Oecologica 23, 213-222 (2002).

281 Herbert, R. J. et al. Artificial coastal lagoons at solar salt-working sites: A network of habitats for specialised, protected and alien biodiversity. Estuarine, Coastal and Shelf Science 203, 1-16 (2018).

282 Walter, W. D., Onorato, D. P. \& Fischer, J. W. Is there a single best estimator? Selection of home range estimators using area-under-the-curve. Movement Ecology 3, 10 (2015).

283 Bellisario, B. Conserving migration in a changing climate, a case study: The Eurasian spoonbill, Platalea leucorodia leucorodia. Biological Conservation 217, 222-231 (2018). 
284 Santini, L., Saura, S. \& Rondinini, C. Connectivity of the global network of protected areas. Diversity and Distributions 22, 199-211 (2016).

285 Phillips, S. J., Anderson, R. P. \& Schapire, R. E. Maximum entropy modeling of species geographic distributions. Ecological Modelling 190, 231-259 (2006).

286 Du Feu, C. R., Clark, J. A., Schaub, M., Fiedler, W. \& Baillie, S. R. The EURING Data Bank-a critical tool for continental-scale studies of marked birds. Ringing \& Migration 31, 1-18 (2016).

287 Farine, D. R. et al. Ecology of woodland songbird social networks: data and insights from large-scale monitoring of individual behaviour. Journal of Animal Ecology (2020).

288 Guillemain, M. et al. What is the spatial unit for a wintering teal Anas crecca? Weekly day roost fidelity inferred from nasal saddles in the Camargue, southern France. Wildlife Biology 16, 215-220 (2010).

289 Tamisier, A. Rythmes nycthéméraux des sarcelles d'hiver pendant leur hivernage en Camargue. Alauda 10, 235-256 (1972).

290 Rubio, L. \& Saura, S. Assessing the importance of individual habitat patches as irreplaceable connecting elements: An analysis of simulated and real landscape data. Ecological Complexity 11, 28-37 (2012).

291 FAO. Radio telemetry and bird movements. [Available at http://www.fao.org/3/a1521e/a1521e07.pdff (2020).

292 Godet, L. \& Devictor, V. What conservation does. Trends in Ecology \& Evolution 33, 720-730 (2018).

293 Gaget, E. et al. Assessing the effectiveness of the Ramsar Convention in preserving wintering waterbirds in the Mediterranean. Biological Conservation 243, 108485 (2020).

294 Environment, M. o. t. Kabukuri-numa and surrounding rice paddies, 2010).

295 Côté, I. M. \& Sutherland, W. J. The Effectiveness of Removing Predators to Protect Bird Populations: Efectividad de la Remoción de Depredadores para Proteger Poblaciones de Aves. Conservation Biology 11, 395-405 (1997).

296 Lilleyman, A. et al. An artificial site provides valuable additional habitat to migratory shorebirds in a tropical harbour. Pacific Conservation Biology 1, 19036 (2020).

297 Sebastián-González, E. \& Green, A. J. Reduction of avian diversity in created versus natural and restored wetlands. Ecography 39, 1176-1184 (2016). 
Bibliography | 175 

Summary 


\section{Summary}

A well-connected network of habitat sites is essential for bird migration. Under rapid habitat loss caused by climate and land use changes, the connectivity of these networks is decreasing, which impacts the bird populations that depend on these networks. Moreover, this also affects other ecological processes that dependent on bird movements, such as disease transmission of avian influenza. Conserving these bird migration networks is crucial for efficient and effective maintenance or restoration of its ecological functions. Although this has been pointed out by scientists 20 years ago, this specific aspect has not yet been included in international conservation efforts for migratory bird species. Conservation can be improved by a better understanding of the causes and consequences of decreasing connectivity in bird migration networks. However, this knowledge remains limited.

Therefore, this thesis advances the understanding of the extent to which environmental changes breakdown bird migration networks, and its potential consequences, such as its effect on population declines and disease transmission. Furthermore, to conserve the connectivity of bird migration networks, it is important to prioritize conservation efforts of sites that have a crucial contribution to the connectivity of the entire network. I propose a quantitative methodological framework for monitoring and understanding of the dynamics of bird migration networks from a local to a flyway scale, which can also assist policy makers when planning conservation actions.

Taking advantage of remote sensing datasets, I quantified habitat loss, fragmentation, and isolation from 1992 to 2012 for eight migratory waterfowl species in the East Asian-Australasian Flyway (Chapter 2). The patterns of habitat degradation differed spatially across the flyway, i.e., wetlands sites degraded more in the southwest of the flyway while wetland availability improved in the northwest. I analysed these differences in habitat degradation over the eight studied species, and found that habitat degradation affected species with different migration extents to a different degree. Migratory species with longer and narrower migration corridors were more affected by habitat degradation, because they had fewer alternative stopover sites at similar latitudes. The species with shorter and broader migration corridors could take advantage of improved habitat conditions in the west of their migration pathway. These findings improve the 
understanding of the variation in effects of environmental changes on different migratory species.

Next to these spatial patterns in habitat degradation, I monitored the changes in functional connectivity of migration networks of the eight waterfowl species in the East Asian-Australasian Flyway between 1992-2015 (Chapter 3). This enabled me to test the degree to which the breakdown of migration networks impacted the population sizes of migratory bird species. I found that the loss of functional connectivity in migration networks significantly predicted population declines in the studied migratory species, while no previously considered species traits could explain these declines. Thus, the decreasing connectivity in migration networks as a consequence of habitat loss and degradation, could negatively and crucially impact population sizes of migratory birds. This novel predictor for population declines of migratory birds provides new insights of the underlying mechanisms that affect population trends of migratory birds under environmental changes.

Thus, conservation of network connectivity is an essential step for safeguarding migratory bird populations, which can be obtained by setting conservation priorities for sites that are more important than others in their contribution to the connectivity of the network. To facilitate the calculation of the importance of a site from a network perspective, I investigated to what extent different network metrics (degree, betweenness, and node resistance) and site-specific habitat loss patterns contributed to the breakdown of migration networks (Chapter 5). By simulating site loss from migration routes of four bird species in the Asian flyways under different scenarios (i.e., in order of degree, betweenness, node centrality, and degree of habitat loss), I observed that site loss in order of the highest to the lowest betweenness value generated a significantly more rapid decrease in connectivity of bird migration networks. According to this conclusion, I identified keystone sites in a network by first selecting sites with a relatively high betweenness value, and then identifying which of those sites was experiencing habitat loss, indicating a higher risk of being removed from the network. I found that $42 \%$ of these keystone sites for the four studied species were not designated as protected areas. These results point out the urgency and importance of prioritizing conservation efforts for keystone sites from a network perspective. 
A better understanding of the dynamics of these migration networks and early-warning signals for network collapse can be obtained from quantifying how environmental factors predict site quality for migratory birds. Using greater white-fronted geese (Anser albifrons) as an indicator species, I tested the extent to which environmental factors (i.e., both anthropogenic and ecological factors) influence their movement patterns at stopover sites in the East Asian-Australasian Flyway (Chapter 4). I found that environmental factors accurately predicted movement patterns (i.e., median movement distance and percentage of flying time) of the geese at stopover sites. Notably, farming activities negatively affected habitat quality for geese, as indicated by their higher frequency and longer distance of local movements in landscapes with a higher proportion of farmlands. These results provide insights into the probability that a site may be lost from a migration network, and can thereby serve as a quantitative tool for monitoring and understanding the dynamics of migration networks of waterfowl species.

Lastly, besides impacts on bird population, I investigated whether the spread of bird-borne infectious diseases was accelerated by changing structures of migration networks under global changes (Chapter 6). I constructed migration networks for 47 bird species (all Anatidae) in the northern hemisphere, and monitored their structural changes from 19502019. This enabled me to map the changes in intra-species and interspecies crossroads between flyways where different populations mix and viruses can change hosts. I observed a northward shift of North American migratory birds, and an eastward shift in Eurasian birds. This may be responsible for an increased intra-species crossing intensity in North America, and an increased inter-species crossing intensity in Eurasia. Thus, the changes in movement networks of migratory hosts driven for example by climate warming and land use changes may contribute to increasing risks of long-distance transmissions and broad-scale outbreaks of infectious diseases.

Combining the evidence and techniques presented in this thesis, I synthesized the various consequences of weakening network connectivity, and proposed a quantitative framework that can enable scientists and policy makers to prioritize site conservation in movement networks (Chapter 7). Decreasing connectivity in migration networks can not only trigger population declines, but also impact multiple functional processes including information diffusion, seed dispersal, predator-prey interactions, pathogen 
transmission, and gene flow. Thus, maintaining or restoring the connectivity of bird movement networks safeguards not only bird populations, but also the ecosystem services provided by their movements. I identified gaps between policy and science, i.e., a minority of criteria in international policies for defining site importance accounted for the connectivity of bird movements, all of which remain qualitative. Thus, I recommend to improve conservation policy making from a network perspective. The proposed methodological framework in this thesis facilitates systematic conservation decisions for movement networks. 



\section{Samenvatting}




\section{Samenvalting}

Een goed verbonden netwerk van habitatplekken is essentieel voor de migratie van vogels. Snel habitatverlies door veranderingen in klimaat en landgebruik zorgt ervoor dat de connectiviteit van deze netwerken afneemt. De verminderde connectiviteit heeft een grote impact op vogelpopulaties en op ecologische processen die afhankelijk zijn van de bewegingen van vogels, zoals de verspreiding van vogelgriep. De bescherming van migratienetwerken is cruciaal voor een efficiënte en effectieve instandhouding of herstel van ecologische functies. Hoewel wetenschappers deze problematiek 20 jaar geleden al aankaartten, werd dit specifieke aspect nog niet meegenomen in internationale natuurbeschermingsmaatregelen voor migrerende vogelsoorten. Een betere begrip van de oorzaken en gevolgen van verminderde connectiviteit van migratienetwerken is dan ook noodzakelijk. Deze kennis is vooralsnog echter beperkt.

Daarom draagt deze thesis bij aan onze kennis rond de mate waarin veranderingen in de leefomgeving leiden tot de afbraak van migratienetwerken en de mogelijke gevolgen hiervan, zoals effecten op de achteruitgang van populaties en de verspreiding van ziektes. Om de connectiviteit van migratiewerken te vrijwaren, is het belangrijk om prioriteit te geven aan locaties die een cruciale bijdrage leveren aan de connectiviteit van het gehele netwerk. Ik stel een kwantitatieve en methodologische omkadering voor om de dynamiek van migratienetwerken te monitoren en beter te begrijpen, zowel op lokale schaal als op het niveau van de volledige trekroute. Deze inzichten kunnen beleidsmakers helpen met het plannen van beschermingsmaatregelen.

Ik kwantificeerde habitatverlies, fragmentatie en isolatie van 1992 tot 2012 voor acht migrerende watervogelsoorten op de Oost-Aziatische en Australazische trekroute met behulp van remote sensing gegevens

(Hoofdstuk 2). De ruimtelijke patronen van habitatdegradatie verschilden langs de trekroute: i.e. natte gebieden waren meer gedegradeerd in het zuidwesten terwijl de beschikbaarheid van deze gebieden verbeterde in het noordwesten. Ik analyseerde deze verschillen in habitatdegradatie voor de acht watervogelsoorten en vond dat soorten met verschillende migratiepatronen op uiteenlopende manieren beïnvloed worden door habitatdegradatie. Soorten met lange, nauwe migratieroutes ondervonden meer hinder van habitatdegradatie, omdat ze minder alternatieve stopplekken hadden op dezelfde breedtegraad. Soorten met korte, brede 
migratieroutes konden voordeel halen uit verbeterde habitatcondities ten westen van hun migratieroute. Deze inzichten verbeteren ons begrip van de diverse effecten die migrerende vogelsoorten ondervinden door veranderingen in hun leefgebied.

Naast de ruimtelijke patronen van habitatdegradatie bestudeerde ik de veranderingen in functionele connectiviteit van migratienetwerken voor acht watervogelsoorten op de Oost-Aziatische en Australazische trekroute tussen 1992 en 2015 (Hoofdstuk 3). Ik onderzocht of de afbraak van migratienetwerken een invloed heeft op de populatiegroottes van migrerende vogelsoorten. Ik ontdekte dat het verlies aan functionele connectiviteit in migratienetwerken de afname in populatiegrootte van de migrerende soorten significant voorspelde, terwijl voorheen bestudeerde soortkenmerken deze afnames niet konden verklaren. Een verminderde connectiviteit in migratienetwerken als gevolg van habitatverlies en -degradatie kan dus een sterk negatief effect hebben op de populatiegroottes van migrerende vogels. Deze nieuwe voorspellende factor voor populatieafnames van migrerende vogels biedt andere inzichten in de onderliggende mechanismen die de populatietrends van migrerende vogels beïnvloeden door veranderingen in hun leefomgeving.

De instandhouding van netwerkconnectiviteit is dus een essentiële stap voor de bescherming van migrerende vogelpopulaties. Dit kan bereikt worden door gebieden die een belangrijke bijdrage leveren aan de connectiviteit van het netwerk prioritair te beschermen. Om de berekening hoe belangrijk een bepaald gebied is vanuit netwerkperspectief te vergemakkelijken, onderzocht ik in welke mate verschillende netwerkeigenschappen (degree, betweenness en node resistance) en gebiedspecifieke patronen van habitatverlies bijdragen aan de afbraak van migratienetwerken (Hoofdstuk 5). Ik simuleerde het verlies van gebieden langs de migratieroutes voor vier vogelsoorten van de Aziatische trekroute aan de hand van verschillende scenario's (i.e., met diverse waarden voor degree, betweenness, node centrality en mate van habitatverlies). Ik ontdekte dat het verlies van een gebied tussen de hoogste en de laagste waarde van betweenness leidde tot de snelste en meest significante afname in connectiviteit van vogelmigratienetwerken. Op basis van deze conclusie, identificeerde ik sleutelgebieden in een netwerk door eerst gebieden met een relatief hoge betweenness-waarde te selecteren en vervolgens te bepalen welke van deze gebieden onderhevig waren aan habitatverlies, wat aangeeft 
dat deze gebieden een hoger risico lopen om uit het netwerk te verdwijnen. Ik vond dat 42 procent van deze sleutelgebieden voor de vier bestudeerde soorten niet als beschermde gebieden bestemd waren. Deze resultaten wijzen op de urgentie en het belang om beschermingsmaatregelen prioritair toe te wijzen aan sleutelgebieden vanuit een netwerkperspectief.

Een beter begrip van de veranderingen in deze migratienetwerken en vroegtijdige alarmsignalen voor het ineenstorten van netwerken kan verkregen worden door te kwantificeren hoe omgevingsfactoren de kwaliteit van een gebied voor migrerende vogels voorspellen. Ik gebruikte kolganzen (Anser albifrons) als indicatorsoort om te testen in welke mate bepaalde omgevingsfactoren (i.e., zowel antropogene als ecologische factoren) de bewegingspatronen op stopplekken langs de Oost-Aziatische en Australazische trekroute beïnvloeden (Hoofdstuk 4). Ik vond dat omgevingsfactoren de bewegingspatronen (i.e., mediane afstand en percentage vliegtijd) van de ganzen op de stopplekken nauwkeurig voorspelden. Het was opmerkelijk dat landbouwactiviteit een negatieve invloed had op de habitatkwaliteit voor ganzen. Dit bleek uit de hogere frequentie en langere afstanden van lokale bewegingen in gebieden met meer landbouwgronden. Deze resultaten bieden inzichten in de waarschijnlijkheid dat een gebied verdwijnt uit een migratienetwerk en kunnen gebruikt worden als een kwantitatieve methode om de veranderingen binnen migratienetwerken van watervogelsoorten te monitoren en te begrijpen.

Tenslotte, naast de impact op vogelpopulaties, bestudeerde ik of de verspreiding van door vogels gedragen infectieziektes versneld wordt door andere structuren in migratienetwerken als gevolg van globale veranderingen (Hoofdstuk 6). Ik bouwde migratienetwerken voor 47 vogelsoorten (alle Anatidae) van het noordelijk halfrond en bekeek structurele veranderingen in deze netwerken tussen 1950 en 2019. Dit liet me toe om de veranderingen van kruispunten in trekroutes binnen soorten en tussen soorten in kaart te brengen. Op deze kruispunten kunnen verschillende populaties samenkomen en kunnen virussen van gastheer wisselen. Ik observeerde een noordwaartse verschuiving van NoordAmerikaanse vogels en een oostwaartse verschuiving van Euraziatische vogels. Deze verschuivingen zijn mogelijk verantwoordelijk voor meer populatievermenging binnen soorten in Noord-Amerika en meer populatievermenging tussen soorten in Eurazië. De wijzingen in bewegingsnetwerken van migrerende gastheren, bijvoorbeeld door 
veranderingen in klimaat of landgebruik, zouden dus kunnen bijdragen aan een toenemend risico van lange-afstand-verspreiding en grootschalige uitbraken van infectieziektes.

Door het bewijs en de technieken in deze thesis te combineren, synthetiseerde ik de diverse gevolgen van verzwakkende netwerkconnectiviteit en stelde ik een kwantitatieve omkadering voor om wetenschappers en beleidsmakers in staat te stellen om bepaalde gebieden in een bewegingsnetwerk prioritair te beschermen (Hoofdstuk 7). Een verminderde connectiviteit van migratienetwerken kan niet alleen populatieafnames veroorzaken, maar heeft ook een impact op meerdere functionele processen, zoals diffusie van informatie, zaadverspreiding, interacties tussen roofdieren en prooien, verspreiding van pathogenen en genetische patronen. Het behouden en herstellen van de connectiviteit in bewegingsnetwerken van vogels beschermd dus niet enkel vogelpopulaties, maar ook ecosysteemdiensten die geleverd worden door hun bewegingen. Ik verduidelijk verschillen tussen beleid en wetenschap, i.e., zeer weinig criteria in internationale beleidsplannen houden rekening met de connectiviteit van vogelbewegingen bij het definiëren van belangrijke gebieden. Daarnaast zijn deze criteria allemaal kwalitatief. Ik geef dus aanbevelingen om natuurbeschermingsbeleid te verbeteren vanuit een netwerkperspectief. De voorgestelde methodologische omkadering van deze thesis vergemakkelijkt de systematische beslissingen voor de bescherming van bewegingsnetwerken. 

Affiliation of Co-authors 


\section{Affiliations of co-authors}

de Boer W. F., Yin S., Grishchenko M., Wang Y., Eikelboom J. A. J. Wildlife Ecology and Conservation Group, Wageningen University and Research, Wageningen, the Netherlands

Prins H. H. T.

Animal Sciences Group, Wageningen University and Research, Wageningen, The Netherlands

\section{Si Y., Gong P.}

Ministry of Education Key Laboratory for Earth System Modelling and Centre for Earth System Science, Tsinghua University, Beijing, China

\section{Zhang W.}

Department of Zoology, University of Oxford, Oxford, UK

\section{Zhang $\mathbf{Y}$.}

College of Biology and the Environment, Nanjing Forestry University, Nanjing, China

\section{Cao L.}

State Key Laboratory of Urban and Regional Ecology, Research Center for Eco-Environmental Sciences, Chinese Academy of Sciences , Beijing, China

Kieboom M., van Lammeren R. J. A.

Laboratory of Geo-Information Science and Remote Sensing, Wageningen University and Research, Wageningen, The Netherlands

Takekawa J. Y.

Suisun Resource Conservation District, Suisun City, USA

\section{Liu Q.}

Network Architectures and Services Group, Delft University of Technology, Delft, The Netherlands

\section{Prosser D. J.}

US Geological Survey Patuxent Wildlife Research Centre, Beltsville, USA van der Jeugd $\mathbf{H}$.

Centre for Avian Migration, Netherland Institute of Ecology, Wageningen, The Netherlands 


\section{Fouchier R. A. M.}

Department of Viroscience, Erasmus Medical Center, Rotterdam, The Netherlands

\section{Guillemain M.}

Office Français de la Biodiversité, Unité Avifaune Migratrice, Arles, France

\section{Caizergues A.}

Office Français de la Biodiversité, Unité Avifaune Migratrice, Nantes, France

\section{Li Z .}

Key Laboratory for Earth Surface Processes of the Ministry of Education, Institute of Ecology, College of Urban and Environmental Sciences, Peking University, Beijing, China

\section{Xu C.}

School of Life Sciences, Nanjing University, Nanjing, China

\section{Thorup K.}

Natural History Museum of Denmark, University of Copenhagen, Copenhagen, Denmark

\section{von Rönn J.}

Swiss Ornithological Institute, Sempach, Switzerland 

Acknowledgements 


\section{Acknowledgments}

After four years' laughter and tears, I am so excited to pass to this last section of my PhD dissertation. I heard too many stories about the frustrations of doing a $\mathrm{PhD}$, but, thanks to all of you I acknowledged here, my $\mathrm{PhD}$ was made easy and enjoyable. Thank you for helping me to unravel the networks of migratory birds as well as to discover my own potential.

Thank you Fred. We overcame obstacles in my projects shoulder by shoulder. You are the person who always showed up first when I encountered difficulties in my research. You are so warm-hearted that you feel my struggles even before I told you. You are a great supervisor who always knew who I should go to when I had doubts. You took care of each minor step in my $\mathrm{PhD}$ so that I could smoothly progress and accomplish my projects successfully and in time. Your encouragements made me fearless throughout my PhD. By the way, your cooking is always amazing, which made me feel at home during the dinners at your place.

Thank you Herbert. You guided me not only in my PhD project, but also in my life. You made up my mind to come to Wageningen when we met five years ago in Tsinghua University, which was a great step in my research career. In Wageningen, I substantially developed my research skills, and grew up from a girl to a woman (as you said). During our discussions, you always refreshed me with your wisdom. You enriched me with your abundant experiences and lively stories. You are such a cheerful supervisor that your hearty laughter swept out my troubles every time.

Thank you Yali. We have worked together for a long time, starting seven years ago. I still remember the first time we met in Tsinghua University, the fieldworks we did together, and hundreds of nice discussions we had. You patiently guided me from the very beginning of my research career when I was 'young and inexperienced'. You are like a big sister who supervised me all the way before I became an independent researcher.

Thank you Peng. You applied for this fantastic project, which allowed me to travel to Wageningen, so that I could work on my $\mathrm{PhD}$ in such a great environment with amazing colleagues and collaborators. Moreover, your expertise in remote sensing and geographical information science provided essential knowledge to accomplish my $\mathrm{PhD}$.

Thank you my WEC colleagues, Andrew, Anouschka, Arnold, Ashok, 
Elke, Erlinda, Faith, Frank, Gerda, Haitham, Helen, Henjo, Ignas, Ivan, Jasper, Jente, Joost, Kenana, Kevin, Khaled, Lana, Lorraine, Martijn, Milena, Mikhail, Mohamed, Nady, Patricia, Patrick, Peter, Pim, Tim, Richard, Ronny, Shyam, Sylvana, Weixuan, Yanning, Yin, Yingying, Yong, Yorick, and Zheng. Together, you created this great environment for both my research and life. Your help enabled me to settle down in Wageningen, solve my questions about research, and even overcome my bad emotions. We exchanged ideas, helped in each other's research, watched wildlife, talked about current affairs, looked after the office plants, enjoyed Christmas dinners, etc. These are all incredible and unforgettable moments for me.

Thank you my Tsinghua colleagues, Boyu, Fei, Jie, Ling, Ruijiao, Wenyuan, Wenzhao, Xueyan, and Zhiyuan. We continued our discussions and collaborations on bird migration. We were never tired of talking with each other, and you inspired me a lot. Your remote supports for both my research and personal development in these four years mean a lot to me.

Thank you my collaborators, Alain, Chi, Diann, Henk, Jan, John, Kasper, Lei, Matthieu, Mattias, Qiang, Ron (Fouchier), Ron (van Lammeren), and Zhouyuan. Without your efforts, I would not have accomplished my projects. Your generous supports and insightful comments were the essential nutrients for my $\mathrm{PhD}$.

Many thanks to all the other collaborators who took part in bird tracking and ringing projects in China, Europe, and North America, which provided an impressive amount of data for my $\mathrm{PhD}$ project.

Thank you Aleksi, Andy, Ben, Josh, Rob, Taej, Thomas, and Ward. I was so grateful to meet you during the last/darkest period of my $\mathrm{PhD}$. You rebuilt my self-confidence and motivation for my research. I believe we will continue our collaborations further and further.

Thank you everyone I met in Wageningen, Anna, Annika, Barbara, Caifang, Carolina, Chunzhe, Danju, Dodo, Federico, Feng, Gracie, Jazz, Jianli, Jing, Jiyao, Jose, Juan Ignacio, Jutta, Kathelyn, Katie, Ke, Lan, Lara, Laura, Leo, Linar, Lourens, Maaike, Mahmoud, Manuel, Marc, Marjolein, Marielos, Markus, Masha, Na, Panpan, Qi, Qing, Qinghua, Quan, Rodrigo, Shanshan, Sophie, Tim, Tomo, Ursula, Xiaohan, Xiaoxiao, Xiaofei, Xiaqu, Xuqing, Yanjun, and Yiran. All our unforgettable parties and pleasant chats were the oxygen for my $\mathrm{PhD}$ life. Special thanks to my special friends who offered me help, cheered me up, brought me warmth, and shared these 
important moments. I love you all.

Thank you my soul mate, Zezhong. Your sweet companionship supports me through all the important transits of my research career, from Beijing to the Netherlands, and until infinite further.

Thank you my mom, my dad, and my grandparents. You are the home in my heart. You always support me without asking anything in return. Your selfless love is the lighthouse and the harbour on the journey of my life. 



\section{Biography}




\section{About the author}

Yanjie Xu was born on 28 May, 1992 in Jiangsu, China. In 2010, she obtained her high school diploma at Taicang Senior High School (Jiangsu, China), after which she started her Bachelor of Environmental Science at the Beijing Forestry University (Beijing, China). For her bachelor thesis, she carried out research about spatial temporal changes in water footprint of crop production in Beijing and index decomposition analysis for the determinants. From 2014 to 2016, She carried out

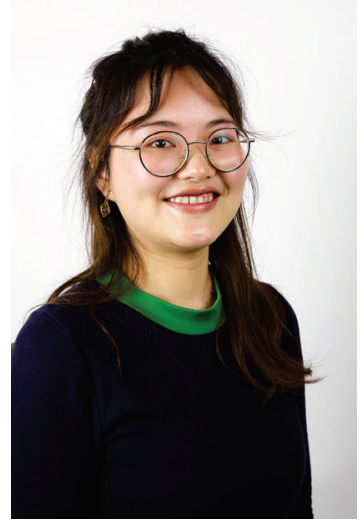
masters research on Global Change Ecology at the Department of Earth System Science, Tsinghua University (Beijing, China). Using techniques of geographical information science and remote sensing, she investigated the role of migratory birds in the global transmission of avian influenza viruses. During this period, she also gained practical skills while working in the field over a three-year period for tracking birds in Asia. Since 2016, She has carried out her $\mathrm{PhD}$ research at the Wildlife Ecology and Conservation Group, Wageningen University and Research (Wageningen, the Netherlands). Her PhD research focuses on ecology and conservation of migratory birds from a local to a global scale. Her work offers novel insights into how climate and land use changes impact wild bird populations. 


\section{Journal publications}

\section{Published}

Xu Y., Si Y., Takekawa J. Y., Liu Q., Prins H. H. T., Yin S, Prosser D. J., Gong P., de Boer W. F. A network approach to prioritize conservation efforts for migratory birds. Conservation Biology 34, 416-426 (2019)

Xu Y., Si Y., Wang Y., Zhang Y., Prins H. H. T., Cao L., de Boer W. F. Loss of functional connectivity in migration networks induces population decline in migratory birds. Ecological Applications 29, e01960 (2019)

Xu Y., Si Y., Yin S., Zhang W., Grishchenko M., Prins H. H. T., Gong P., de Boer W. F. Species-dependent effects of habitat degradation in relation to seasonal distribution of migratory waterfowl in the East Asian-Australasian Flyway. Landscape Ecology 34, 243 (2019)

Xu Y., Gong P., Wielstra B., Si Y. Southward autumn migration of waterfowl facilitates cross-continental transmission of the highly pathogenic avian influenza H5N1 virus. Scientific Reports 6, 30262 (2016)

Xu Y., Huang K., Yu Y., Wang X. Changes in water footprint of crop production in Beijing from 1978 to 2012: a logarithmic mean Divisia index decomposition analysis. Journal of Cleaner Production 87, 180-187 (2015)

Si Y., Xu Y., Xu F., Li X., Zhang W., Wielstra B., Wei J., Liu G., Luo H., Takekawa J. Y., Sivananintha B., Zhang T., de Boer W. F., Prins H. H. T., Gong P. Spring migration patterns, habitat use, and stopover site protection status for two declining waterfowl species wintering in China as revealed by satellite tracking. Ecology and Evolution 8, 6280-6289 (2018)

Wang Y. X. G., Matson K. D., Xu Y., Prins H. H. T., Huang Z. Y. X., de Boer W. F. Habitat connectivity, host assemblage characteristics of local and neighbouring counties, and temperature jointly shape the spatial expansion of Lyme disease in United States. Remote Sensing 11, 2354 (2019)

Li Z., Wang Z., Liu X., Fath B. D., Liu X., Xu Y., Hutjes R., Kroeze C. Causal relationship in the interaction between land cover change and underlying surface climate in the grassland ecosystems in China. Science of the Total Environment 647, 1080-1087 (2018)

Wang X., Huang K., Yu Y., Hu T., Xu Y. An input-output structural decomposition analysis of changes in sectoral water footprint in China. Ecological Indicators 69, 26-34 (2016).

Hu T., Huang K., Yu Y., Zhang X., Xu Y., Wang X. Measuring water footprint on a lake basin scale: a case study of Lake Dianchi, China. CLEAN-Soil, Air, Water 44, 1296-1305 (2016). 


\section{In press/revision}

Li Z., Xu Y., Sun Y., Wu M., Zhao B. Urbanization-Driven Changes in LandClimate Dynamics: A Case Study of Haihe River Basin, China. Remote Sensing [In Press].

Xu Y., Kieboom M., van Lammeren R. J. A., Si Y., de Boer W. F. Indicators of site loss from a migration network: anthropogenic factors influence waterfowl movement patterns at stopover sites. Global Ecology and Conservation [Under Review].

Yin S., Xu Y., Batbayar N., Takekawa J. Y., Si Y., Prosser D. J., Newman S. H., Prins H. H. T., de Boer W. F. Do contrasting patterns of migration movements and disease outbreaks between congeneric waterfowl species reflect differing immunity? Geospatial Health [Under Review].

Li Z., Zhang H., Xu Y., Wang S. Averaged body size, rather than biodiversity, drives avian community stability over North America. Functional Ecology [Under Review]. 
Biography | 203 



\section{PE\&RC Training and Education Statement}




\section{PE\&RC Training and Education Statement}

With the training and education activities listed below the $\mathrm{PhD}$ candidate has complied with the requirements set by the C.T. de Wit Graduate School for Production Ecology and Resource Conservation The C.T. De Wit
Gradunte Shool
PEERCODUCTION (PE\&RC) which comprises of a minimum total of 32 ECTS $(=22$ weeks of activities)

\section{Review of literature (4.5 ECTS)}

The ecology of bird migration

\section{Writing of Project proposal (4.5 ECTS)}

Seasonal migration networks of east Asian waterfowl: crucial stopover sites, connectivity, and collapse

\section{Post-graduate courses (15 ECTS)}

- Animal movement analysis; PE\&RC (2017)

- Consumer-resource interactions; PE\&RC (2018)

- Machine learning for spatial data; PE\&RC (2018)

- Agent-based modelling with NetLogo; PE\&RC (2019)

- The art of modelling; PE\&RC (2019)

\section{Laboratory training and working visits (4.5 ECTS)}

Structures of movement network; University of Oxford (2020)

\section{Invited review of (unpublished) journal manuscript (3 ECTS)}

- PLoS One: bird migration and disease transmission (2016)

- PLoS One: disease transmission network (2019)

- PLoS One: predator-prey interaction in birds (2019) 
Deficiency, refresh, brush-up courses (3 ECTS)

Ecological methods; WUR (2018)

\section{Competence strengthening / skills courses (1.6 ECTS)}

- Career perspectives; WGS (2019)

- Writing propositions for your PhD; WGS (2019)

- Last stretch of the PhD programme; WGS (2019)

\section{Scientific integrity / ethics in science activity (0.4 ECTS)}

- Ethics in plant and environmental sciences; SENSE (2018)

- Scientific integrity; SENSE (2020)

PE\&RC Annual meetings, seminars and the PE\&RC weekend (1.8 ECTS)

- PE\&RC Weekend for first years (2017)

- PE\&RC Weekend for last years (2019)

\section{Discussion groups / local seminars / other scientific meetings (6.6 ECTS)}

- Wageningen Evolution and Ecology Seminars (WEES) (2016)

- Current themes in ecology ecological networks - networks in ecology (2016)

- R Users meeting; PE\&RC discussion group (2017)

- Symposium: frontiers in resilience research (2017)

- Landscape dynamics discussion group (2018)

- Reading club for modelling to inform infectious disease (2018)

\section{International symposia, workshops and conferences (6.4 ECTS)}

- Annual Meeting of Waterbird Society; Iceland (2017)

- Netherlands Annual Ecology Meeting; the Netherlands (2018)

- British Ecological Society Annual Meeting; United Kingdom (2019) 
Netherlands Annual Ecology Meeting; the Netherlands (2020)

\section{Lecturing / supervision of practicals / tutorials (4 ECTS)}

- Ecological methods I $(2018,2019)$

\section{Supervision of MSc students (3 ECTS)}

- Using movement patterns of migratory birds to predict node quality in migration networks 


\section{Colophon}

Cover designed by Yanjie Xu.

The research described in this thesis was financially supported by the China Scholarship Council.

Financial support from Wageningen University for printing this thesis is gratefully acknowledged.

Lay-out and print by: ProefschriftMaken || www.proefschriftmaken.nl 
